\title{
Osteology of Klamelisaurus gobiensis (Dinosauria, Eusauropoda) and the evolutionary history of Middle-Late Jurassic Chinese sauropods
}

\author{
Andrew J. Moore ${ }^{\mathrm{a}, \mathrm{b}}$, Paul Upchurch ${ }^{\mathrm{c}}$, Paul M. Barrett ${ }^{\mathrm{d}}$, James M. Clark ${ }^{\mathrm{a}}$ and Xu Xing \\ ${ }^{a}$ Department of Biological Sciences, The George Washington University, Washington, DC 20052, \\ USA; ${ }^{b}$ Department of Anatomical Sciences, Stony Brook University, Stony Brook, NY \\ 11794-8081, USA; ' Department of Earth Sciences, University College London, London WC1E \\ 6BS, UK; ${ }^{\text {d}}$ Department of Earth Sciences, Natural History Museum, London SW7 5BD, UK; \\ ${ }^{\mathrm{e}}$ Key Laboratory of Vertebrate Evolution and Human Origins, Institute of Vertebrate \\ Paleontology and Paleoanthropology, Chinese Academy of Sciences, Beijing 100044, People's \\ Republic of China
}

(Received 23 March 2019; accepted 3 April 2020)

Fossil-rich deposits from the Middle and Late Jurassic of China have yielded a diverse array of sauropod dinosaurs, including numerous species referred to Mamenchisaurus and Omeisaurus. Despite an abundance of fossils and a proliferation of taxa, the anatomy of Middle-Late Jurassic Chinese sauropods remains poorly documented. Here, we comprehensively redescribe and illustrate Klamelisaurus gobiensis from the Middle-Late Jurassic Shishugou Formation of northwest China. Phylogenetic analyses conducted under parsimony and time-calibrated Bayesian optimality criteria consistently recover Klamelisaurus as a member of a predominantly Chinese radiation of exceptionally long-necked eusauropods that includes Mamenchisaurus spp., Chuanjiesaurus, Qijianglong and Wamweracaudia. In most analyses, this lineage also includes Euhelopus, reviving a 'traditional' Euhelopodidae and calling into question the macronarian affinities of Euhelopus. Klamelisaurus shares several features with Euhelopus that are unique to a subset of East Asian taxa or rare among sauropods, including a convex ventral margin of the prezygodiapophyseal lamina in middle-posterior cervical vertebrae, a ventrally bifurcated postzygodiapophyseal lamina in posterior cervical vertebrae, and development of a rugose projection extending anteriorly from the epipophysis into the spinodiapophyseal fossa in most cervical vertebrae. Anatomical comparisons of the cervical vertebrae of Klamelisaurus to several other sauropodomorphs and insights from myological studies of extant archosaurs strongly suggest that this latter structure, often considered part of an epipophyseal-prezygapophyseal lamina, is an epaxial muscle scar that is distinct from pneumatic structures of the lateral surface of the neural spine. The phylogenetic and comparative anatomical data presented here provide a foundation for future revision of the taxonomy and systematics of sauropods from the Junggar and Sichuan basins.

Keywords: sauropod; Mamenchisauridae; Euhelopodidae; pneumaticity; Bayesian phylogenetics 


\section{Introduction}

The first dinosaur formally described from China was a sauropod (Wiman 1929), and subsequent decades of discovery have resulted in the erection of dozens of sauropod genera from the Jurassic and Cretaceous of East Asia (e.g. Young 1937, 1939, 1954; Yeh 1975; Dong et al. 1983; Dong \& Tang 1984; Dong 1990; Zhao 1993; Martin et al. 1994; Martin-Rolland 1999; Fang et al. 2000; Tang et al. 2001; Ouyang \& Ye 2002; You et al. 2003, 2006, 2008; Peng et al. 2005; Ye et al. 2005; Ksepka \& Norell 2006; X. Wang et al. 2007; L€u et al. 2008, 2009; X. Zhang et al. 2009; K. Li et al. 2010; Mo et al. 2010; Sekiya 2011; Wu et al. 2013; Xing et al. 2013, 2015; Ren et al. 2018; X. Xu et al. 2018; Zhou et al. 2018). The Middle and Late Jurassic strata of the Sichuan and Junggar basins are particularly rich in sauropod fossils, with no fewer than 14 genera, including eight species of Omeisaurus (Young 1939, 1958; He et al. 1988; Tang et al. 2001; Jiang et al. 2011; Tan et al. 2020) and six species of Mamenchisaurus (Young 1954; Young \& Zhao 1972; Dong et al. 1983; Russell \& Zheng 1993; He et al. 1996; Pi et al. 1996; Y. Zhang et al. 1998), named from the Shishugou, Shaximiao, Qiketai and Suining formations. Omeisaurus and Mamenchisaurus are often included as outgroups in phylogenetic analyses of neosauropod lineages, but despite an abundance of fossils, the anatomy of these and related taxa from the Middle-Late Jurassic of China remain poorly documented in the literature and very little concerted effort has been made to clarify their taxonomy or evolutionary interrelationships (Sekiya 2011; Xing et al. 2015; Ren et al. 2018).

Focused inquiry into Middle-Late Jurassic Chinese eusauropods - often considered to constitute an endemic lineage termed Mamenchisauridae (e.g. He et al. 1988; Russell 1993; Sekiya 2011; Xing et al. 2015) or Euhelopodidae (e.g. Upchurch 1995) - has the potential to clarify our understanding of early eusauropod evolution and begin to answer several outstanding questions in sauropod biology and palaeobiogeography. Perhaps the most striking observation on these East Asian forms is that they exhibit profound convergence with later neosauropod radiations in absolute neck elongation and the extent of postcranial skeletal pneumatization. Necks greater than $9 \mathrm{~m}$ in length occur in several groups of sauropod dinosaurs, including mamenchisaurids, diplodocids and macronarians (Wedel 2006; Taylor \& Wedel 2013). Although total neck length is known for relatively few sauropods, mamenchisaurids are bracketed phylogenetically by several taxa with necks less than $4 \mathrm{~m}$ in length (e.g. Shunosaurus, Cetiosaurus, Jobaria, Camarasaurus; AJM, unpublished data), suggesting that the extremely long necks of some mamenchisaurids evolved independently of those found within Neosauropoda. The data available at present thus indicate that mamenchisaurids were the first lineage to evolve hyper-elongate necks and were the only nonneosauropod group to do so. As in other particularly long-necked sauropod groups (Wedel et al. 
2000; Wedel 2003; Taylor \& Wedel 2013), the evolution of extreme neck elongation in these Middle-Late Jurassic East Asian taxa is accompanied by the development of lightly constructed, highly pneumatic vertebrae (Young \& Zhao 1972; Russell \& Zheng 1993; Ouyang \& Ye 2002), although details of their internal pneumatic architecture have never been closely assessed. Focused comparative study of mamenchisaurid axial columns alongside those of other particularly long-necked sauropod lineages will undoubtedly shed light on the suite of morphological prerequisites necessary to achieve exceptionally long necks and will allow critical assessment of the extent to which different sauropod clades converged on similar solutions to extreme neck elongation.

Comprehensive treatments of the anatomy and systematics of Middle-Late Jurassic East Asian eusauropods are also critical to inquiries into the species richness and palaeobiogeography of sauropod faunas. The alpha diversity of sauropods from Middle-Late Jurassic Chinese deposits appears to rival that of penecontemporaneous Late Jurassic sauropods from western North America (Ikejiri 2005; Harris 2006; Tschopp et al. 2015a; Moore et al. 2018; Mannion et al. 2019), as recent radiometric dating of detrital zircons from beds of the lower Shaximiao Formation at Dashanpu now places the entire Shaximiao Formation - and thus the vast majority of taxa considered to comprise Mamenchisauridae - within the Late Jurassic (J. Wang et al. 2018). However, in the absence of specimen-level phylogenetic and comparative analyses, this perceived diversity may be an artifact of a perfusion of undiagnosable taxa rather than a genuine indication of high global diversity of Late Jurassic sauropods. In addition, a refined understanding of the evolutionary relationships of Middle-Late Jurassic Chinese eusauropods is pertinent to testing hypotheses concerning the isolation of East Asia from western Laurasia and Gondwana at this time (Russell 1993; Upchurch 1995; Barrett et al. 2002; Upchurch et al. 2002; Wilson \& Upchurch 2009; Poropat et al. 2016; X. Xu et al. 2018) and the palaeobiogeographical history of early branching sauropods and eusauropods more broadly.

Inclusion of these Middle-Late Jurassic East Asian eusauropods in broader morphofunctional and palaeobiogeographical analyses first requires that historically and taxonomically important specimens be thoroughly redescribed and that the taxonomy and systematics of the many named species be evaluated critically. Here, we take a modest step towards this aim by redescribing and illustrating Klamelisaurus gobiensis Zhao, 1993, from the Middle-Late Jurassic Shishugou Formation of northwest China. We differentiate Klamelisaurus from the other sauropods found in the Shishugou Formation and, for the first time, test its phylogenetic affinities. We also reexamine the evolutionary history of Middle-Late Jurassic and Early Cretaceous East Asian eusauropods and use insights from comparative study of Klamelisaurus and other sauropods to revise hypotheses concerning the homology of the epipophysealprezygapophyseal lamina. 
Institutional abbreviations

AMNH, American Museum of Natural History, New York, USA; BP, Evolutionary Studies Institute, University of the Witwatersrand, Johannesburg, South Africa; BYU, Brigham Young University Museum of Paleontology, Provo, USA; CCG, Chengdu University of Technology, Chengdu, China; CL, Cuesta Lonsal, specimens deposited in Museo Paleontologico de Galve, Galve, Spain; CM, Carnegie Museum of Natural History, Pittsburgh, USA; CPP, Centro de Pesquisas Paleontologicas Lewellyn Price, Peiropolis, Brazil; DFMMh, Dinosaurier-Freilichtmuseum Münchehagen/Verein zur Förderung der Niedersächsischen Paläontologie e.V., Rehburg-Loccum, OT Münchehagen, Germany; FCPT-D, Fundacion Conjunto Paleontologico de TeruelDinopolis, Teruel, Spain (plus CPT for the fossil material deposited in the museum [Museo Fundacion Conjunto Paleontologico de Teruel]); IVPP, Institute of Vertebrate Paleontology and Paleoanthropology, Beijing, China; LCD/Lfch, World Dinosaur Valley, Lufeng County, Yunnan Province, China; LEICT, New Walk Museum, Leicester,UK; MB.R., Museum für Naturkunde, Berlin, Germany; MCNV, Museo de Ciencias Naturales, Valencia, Spain; MNBH, Musee National Boubou Hama, Niamey, Niger; NHMUK, Natural History Museum, London, UK; PMU, Palaeontological Museum, Uppsala, Sweden; QJGPM, Qijiang Petrified Wood and Dinosaur Footprint National Geological Park Museum, Chongqing, China; SMA, Sauriermuseum Aathal, Switzerland; TMM, Texas Memorial Museum, Austin, USA; USNM, National Museum of Natural History, Washington, DC, USA; UW, University of Wyoming Geological Museum, Laramie, USA; YPM, Yale Peabody Museum, New Haven, USA; ZDM, Zigong Dinosaur Museum, Dashanpu, China; ZH, Museo Paleontologico de Galve, Galve, Spain; ZNM, Zhejiang Natural Museum, Hangzhou, Zhejiang, China.

Anatomical abbreviations

4t, fourth trochanter; ac, acromion; ACDL, anterior centrodiapophyseal lamina; ACPL, anterior centroparapophyseal lamina; acr, acromial ridge; adp, anterodistal process; ag, attachment groove; alp, anterolateral process; amp, anteromedial process; ap, aliform process; as, accessory strut; bb, bulge for the $\mathrm{m}$. biceps brachii; bif. PODL, bifurcated postzygodiapophyseal lamina; bif. SPDL, bifurcated spinodiapophyseal lamina; bu, bump; cc, cnemial crest; CDF, centrodiapophyseal fossa; cf, coracoid foramen; CPOF, centropostzygapophyseal fossa; CPOL, centropostzygapophyseal lamina; CPRF, centroprezygapophyseal fossa; CPRL, centroprezygapophyseal lamina; CPRL-F, fossa of the centroprezygapophyseal lamina; cs, costal spur; D, dorsal vertebra; dlb, distolingual boss; dp, diapophysis; dpc, deltopectoral crest; ems, epipophyseal muscle scar; ep, epipophysis; EPRL, epipophyseal-prezygapophyseal lamina; fc, fibular condyle; fo, fossa; for, foramen; gl, glenoid; gr, groove; icg, intercondylar groove; ior, interosseous ridge; isp, ischial peduncle; k, ventral keel; lr, lateral ridge; md, medial deflection of proximal femur; 
ml, mineralized ligament; mr, medial ridge; os, oblique strut; p, pit; PACDF, parapophyseal centrodiapophyseal fossa; PACPRF, parapophyseal

centroprezygapophyseal fossa; PCDL, posterior centrodiapophyseal lamina; PCDL-F, posterior centrodiapophyseal lamina fossa; PCPL, posterior centroparapophyseal lamina; PCPL-F, posterior centroparapophyseal lamina fossa; pems, pre-epipophyseal muscle scar; pl, dorsolaterally facing platform; pms, paramarginal sulcus; pne, pneumatic excavation; pnf, pneumatic foramen; pnfo, pneumatic fossa; POCDF, postzygapophyseal centrodiapophyseal fossa; PODL, postzygodiapophyseal lamina; PODL-F, postzygodiapophyseal lamina fossa; pop, posterior process; POSDF, postzygapophyseal spinodiapophyseal fossa; POSL, postspinal lamina; poz, postzygapophysis; pp, parapophysis; PPDL, paradiapophyseal lamina; pr, projection; PRCDF, prezygapophyseal centrodiapophyseal fossa; PRDL, prezygodiapophyseal lamina; prep, pre-epipophysis; PRPADF, prezygapophyseal paradiapophyseal fossa; PRPL, prezygoparapophyseal lamina; PRSDF, prezygapophyseal spinodiapophyseal fossa; PRSL, prespinal lamina; prz, prezygapophysis; pup, pubic peduncle; pyr, pyramidal boss; rcb, low rugosity for $\mathrm{m}$. coracobrachialis; rds, rugosity for $\mathrm{m}$. deltoideus scapularis; ri, ridge; rld, rugosity for $\mathrm{m}$. latissimus dorsi; rug, rugosity; $\mathbf{S}$, sacral; sd, shelf-like depression; SDF, spinodiapophyseal fossa; se, sheet-like extensions of the spinoprezygapophyseal lamina; sp, spur; SPDL, spinodiapophyseal lamina; SPOF, spinopostzygapophyseal fossa; SPOL, spinopostzygapophyseal lamina; SPRF, spinoprezygapophyseal fossa; SPRL, spinoprezygapophyseal lamina; sps, smooth pneumatic surface of SDF; str, striations; tc, tibial condyle; td, trough-like depression of the diapophysis; TPOL, interpostzygapophyseal lamina; TPRL, interprezygapophyseal lamina.

\section{Systematic palaeontology}

Dinosauria Owen, 1842

Saurischia Seeley, 1887

Sauropodomorpha Huene, 1932

Sauropoda O. C. Marsh, 1878

Eusauropoda Upchurch, 1995

Klamelisaurus Zhao, 1993

Type species. Klamelisaurus gobiensis (by monotypy).

Diagnosis. As for type and only species (see below). 
Occurrence and age. Jiangjunmiao area, Junggar Basin, Xinjiang Uyghur Autonomous Region, northwest China. Between 1981 and 1985, the Institute of Vertebrate Paleontology and Paleoanthropology (IVPP) cooperated with the Chinese National Academy of Sciences (Academia Sinica) and the Xinjiang Office of Petroleum in a joint research endeavour on the evolution of the Junggar Basin and its petroleum resources. In 1982, the team discovered a sauropod skeleton weathering out in the Jiangjunmiao area, and in 1984 a field crew from the IVPP continued the systematic collection and excavation of the specimen. The specimen was subsequently transported to Beijing and, in 1985, preparation and restoration commenced, although the specimen deteriorated due to extreme fluctuations in ambient temperature and humidity (Zhao 1993).

According to Zhao (1993), Klamelisaurus was recovered from a site $35 \mathrm{~km}$ north of the now-abandoned town of Jiangjunmiao, south of the Kelameili Mountains - its namesake - in the eastern Junggar Basin (Fig. 1). Zhao (1993) noted that the specimen was collected from the grey-brown, purple-red sandy mudstones at the top of what was formerly called the 'Wucaiwan Formation' and is now considered to constitute the lower beds of the Shishugou Formation (Clark et al. 2006). Lithostratigraphical correlation places Klamelisaurus below a tuff from the middle beds of the Shishugou Formation at Wucaiwan approximately $95 \mathrm{~km}$ away, which has been radiometrically dated to $162.2 \pm 0.2 \mathrm{Ma}$ (Choiniere et al. 2013; Han et al. 2015), indicating that Klamelisaurus is likely late Callovian in age (see Moore et al. 2018).

Klamelisaurus gobiensis Zhao, 1993 (Figs 2-24, 34, 36)

Holotype. IVPP V9492, a partial postcranial skeleton including nine middle and posterior cervical vertebrae, 12 dorsal vertebrae, six sacral vertebrae, fragments of 19 caudal vertebrae, four middle-distal chevrons, a proximal dorsal rib, right scapulocoracoid, right humerus, right ulna, right radius, partial left ilium, partial right ischium, a largely complete right femur and distal left femur, a partial right tibia and proximal left tibia, a partial right fibula and proximal left fibula, a partial right astragalus, two metatarsals and five pedal phalanges. The chevrons, distal left femur and elements of the left crus were not mentioned in the original description. The fragmentary teeth, two dorsal ribs, two carpals, a few caudal elements (see below) and the right calcaneum described by Zhao (1993) could not be located. In addition, we reinterpret the metapodial elements that Zhao (1993) attributed to the manus as pedal elements.

Previous diagnoses. The diagnosis offered by Zhao (1993) does not describe unique characteristics of Klamelisaurus, but enumerates features that are broadly distributed in sauropods or ambiguously present in Klamelisaurus: relatively large size; 
opisthocoelous cervical vertebrae; relatively high cervical neural spines; 13 dorsal vertebrae; shallow pleurocoels in the dorsal vertebrae; simple lamination of dorsal vertebrae; expanded neural spine apices in posterior dorsal vertebrae; five sacral vertebrae; fused sacral centra; fusion of the four anterior sacral neural spines; 60 caudal vertebrae; procoelous anterior caudal vertebrae; caudal neural spines that are claviform in shape and strongly posteriorly oblique; gently amphicoelous middle and posterior caudal vertebrae; elongate scapula; small coracoid; humerus with a proximal end that is thick and broad; ulna that is longer than the radius; relatively straight radius; robust ilium lacking a distinct 'dorsal laminar ridge'; anteriorly positioned pubic peduncle of the ilium; robust pubis; slender ischium; a thick and flat femur; and a tibia that is shorter than the fibula. Though not listed in his diagnosis, Zhao (1993) suggested that the proximal end of the ulna is autapomorphic in its degree of expansion; as discussed below, however, the robusticity of the proximal end of the ulna is typical of several contemporaneous sauropods. Upchurch et al. (2004) stated that Klamelisaurus is diagnosed by fusion of the three posterior-most cervical neural spines; however, fusion of the cervical neural spines is not present in Klamelisaurus. Zhao (1993) described two of the cervical centra of Klamelisaurus as fused; he did not include this observation in his diagnosis or suggest it to be autapomorphic, but fusion of cervical vertebrae would be unusual among sauropods. We were unable to confidently identify fused cervical centra in Klamelisaurus.

Emended diagnosis. A eusauropod diagnosed by the following unique autapomorphies: scabrous, sheet-like anterior extensions of the SPRLs in middleposterior cervical vertebrae; oblong, blind pneumatic fossa just anterior to the SDF in middle-posterior cervical vertebrae; triplicate PCPLs in some posterior dorsal vertebrae; dorsally bifurcated SPDL in middle and posterior dorsal vertebrae without confluence with either the SPRL or the SPOL; shelf-like embayment of the proximomedial corner of the anterior face of the humerus, bounded medially by a bulge; and flange-like ridge overhanging the medial surface of the shaft of metatarsal I.

Differential diagnosis. Here, we demonstrate that Klamelisaurus gobiensis is not synonymous with any of the other named Shishugou Formation sauropods.

Bellusaurus sui has been proposed as a juvenile form of Klamelisaurus (Paul 2010), based on their co-occurrence and the juvenile status of all known Bellusaurus material. Moore et al. (2018) provided a detailed comparison of these taxa, showing that Bellusaurus can be distinguished from Klamelisaurus by numerous features, many of which are not readily explainable by differences in ontogenetic stage. In addition to the list of characters offered by Moore et al. (2018) and the autapomorphies given above, we add that Klamelisaurus can be distinguished from Bellusaurus in having: ventral bifurcation of the PODL in posterior cervical vertebrae; a tuberosity for the attachment 
of the $\mathrm{m}$. coracobrachialis in the proximal anterior fossa of the humerus; a dorsally convex anteromedial process of the ulna that slopes well below the level of the olecranon (this surface is essentially flat in Bellusaurus); and an acute angle between the anteromedial and anterolateral processes of the ulna (this angle is $>80$ in Bellusaurus).

Anatomical overlap between Mamenchisaurus sinocanadorum and Klamelisaurus is limited. Both taxa preserve teeth, but the isolated teeth included in the holotype of Klamelisaurus by Zhao (1993) could not be located. Both taxa are reported to have denticulate teeth, but denticles are weakly developed and limited to the posterior dentition in Mamenchisaurus sinocanadorum (Russell \& Zheng 1993; see below). Preserved cervical vertebrae are from non-overlapping parts of the cervical series in the two taxa. Both the anterior cervical vertebrae of Mamenchisaurus sinocanadorum and the middle-posterior cervical vertebrae of Klamelisaurus exhibit centra with an anteriorly restricted lateral pneumatic excavation that is crossed by an anterodorsally orientated septum, although this feature is more broadly distributed in Middle-Late Jurassic Chinese sauropods. The differing geological ages of Mamenchisaurus sinocanadorum and Klamelisaurus imply taxonomic separation, as Klamelisaurus is from the lower Shishugou Formation (late Callovian) while Mamenchisaurus sinocanadorum was collected from its middle or upper beds (Oxfordian; Moore et al. 2018). In addition, Mamenchisaurus sinocanadorum is inferred to have had a much longer neck than Klamelisaurus: although the holotype and only specimen of Mamenchisaurus sinocanadorum preserves only the first four cervical vertebrae, these elements are slightly longer than those of Omeisaurus tianfuensis and the holotype of Mamenchisaurus hochuanensis, both of which have substantially longer necks than Klamelisaurus (Supplementary Fig. 1). More robust differentiation of these taxa awaits additional fossil discoveries.

Klamelisaurus can be distinguished from Tienshanosaurus chitaiensis Young, 1937 in having: a lateral pneumatic opening of the cervical centra that occupies less than the anterior two-thirds of the centrum; procoelous anterior caudal vertebrae; anterior caudal neural spines with an anterodorsal margin that is level with or posterior to the posterior margin of the postzygapophyses; a straight, rather than concave, posterior margin of the dorsal part of the acromion; and a coracoid that lacks a marked infraglenoid notch and lip.

\section{Description and comparisons}

All descriptions were made directly from the holotype of Klamelisaurus. Comparisons with other taxa were made from direct observations of specimens or from published descriptions, illustrations and photographs (see Supplementary Table 1). Terminology 
for the vertebral laminae follows Wilson $(1999,2012)$ and that of vertebral fossae follows Wilson et al. (2011), although we adopt the change in prefix proposed by Tschopp \& Mateus (2013) for the TPRL and TPOL.

Except where noted, we use avian myological terminology in interpreting muscle attachments on the axial skeleton of Klamelisaurus, based principally on the work of Wedel \& Sanders (2002), Tsuihiji (2005, 2007), Organ (2006), Schwarz-Wings (2009) and Taylor \& Wedel (2013).

Accurate measurement of the preserved material was complicated by the large size of most elements and by extensive reconstruction of some regions of the skeleton. Zhao (1993) provided linear length measurements for the cervical vertebrae and length and total height measurements for the dorsal vertebrae. Three of us (AJM, PU \& PMB) took linear measurements of IVPP V9492 using both Fowler electronic callipers (accurate to $0.01 \mathrm{~mm}$, for distances up to $307 \mathrm{~mm}$ ) and measurement tape. Where a measurement was taken more than once, we report the average value. Because most vertebral elements of Klamelisaurus are preserved in articulation with at least one other vertebra, and because the anterior articular surface of the centrum, when exposed, is highly reconstructed, we only report functional centrum length, i.e. the midline distance between the rim of the anterior condyle and the ventral edge of the posterior cotyle.

Phylogenetic definitions of the clade names used in this study are given in Supplementary Table 2. Pending much-needed revision of Middle-Late Jurassic East

Asian eusauropods, we use the term 'Core Mamenchisaurus-like Taxa' to refer to those taxa that are found to be closely related across all of our phylogenetic analyses (see below). This group includes Klamelisaurus, Qijianglong, species of Mamenchisaurus (with the exception of the labile M. constructus), the holotype and referred specimens of Chuanjiesaurus, Wamweracaudia, an isolated series of three cervicodorsal vertebrae from the Shishugou Formation, and the unnamed Phu Kradung taxon (Suteethorn et al. 2013).

\section{General comments}

The elements of Klamelisaurus gobiensis (IVPP V9492) were reconstructed and prepared for mounting sometime before photographs were taken for Zhao (1993, pl. I). Many elements are still partially encased in metal armature and nearly all have been at least partially reconstructed and painted, often obscuring the original morphology and requiring cautious interpretation; we are explicit in the following description and in 
related figures about what portions of these elements are reliably unaltered. At least some elements have been

modified since the original images were taken, as reflected in Zhao (1993, pl. I, fig. 1): a reconstructed anterior process of the cervical rib has been added since this image was taken, and the fabricated posterior connection of the cervical rib to the centrum has since been removed.

\section{Ontogenetic age}

Wedel \& Taylor (2013a) provided seven features that, considered in toto, likely indicate skeletal maturity in sauropods. One of these, presence of an external fundamental system in the cortices of long bones, has yet to be assessed in Klamelisaurus. Another, absolute size, is not necessarily strong evidence for or against skeletal maturity (as noted by Wedel \& Taylor 2013a), and Klamelisaurus is intermediate in size among Middle-Late Jurassic Chinese eusauropods (Supplementary Table 3; Supplementary Fig. 1). Most of the remaining features of skeletal maturity listed by Wedel \& Taylor (2013a) are present in Klamelisaurus: Klamelisaurus lacks open neurocentral sutures; shows complete, or nearly complete, fusion of the sacral vertebrae (although the presence of a sacricostal yoke cannot be assessed, as no sacral ribs are preserved); has cervical ribs that are fused to the neural arches and centra; and shows fusion of the scapula and coracoid. Metaplastically mineralized vertebral ligaments and tendons are not unambiguously present in

Klamelisaurus, although it is possible that one is preserved in dorsal vertebra 12 (see below), and the well-developed muscle scars of the cervical series may be related to the age of the specimen, as has previously been interpreted for the 'pre-epipophyseal ridges' on the dorsal surface of the postzygapophyses in Camarasaurus lewisi (McIntosh et al. 1996). Based on these observations, and pending histological assessment of the specimen, we consider Klamelisaurus to be skeletally mature.

\section{Dentition}

The fragmentary teeth described by Zhao (1993) could not be located. Zhao (1993) did not explain his inclusion of these teeth in the holotype of Klamelisaurus, which otherwise consists of a largely intact vertebral column and appendicular elements. Zhao (1993) described the teeth as 'spoon-shaped', with relatively thin crowns bearing smooth, glossy, dark brown enamel with grey-white wear facets. According to Zhao (1993), the teeth lack a distinct medial ridge (presumably of the lingual surface) and exhibit well-developed denticles. An apicobasal lingual ridge is plesiomorphic for eusauropods (Barrett et al. 2002) and is present in at least some teeth in other described 
Core Mamenchisaurus-like Taxa (Mamenchisaurus sinocanadorum - IVPP V10603; Mamenchisaurus youngi - Ouyang \& Ye 2002; Omeisaurus tianfuensis - He et al. 1988) and Euhelopus (PMU 24705/1a-b). Among sauropods with spatulate teeth, an apicobasal lingual ridge is otherwise absent only in Jobaria (unnumbered MNBH TIG specimens housed at University of Chicago), teeth referred to Hudiesaurus (IVPP V11121-2; Dong 1997) and Oplosaurus armatus (Upchurch et al. 2004, 2011); if the teeth described by Zhao (1993) indeed belong to Klamelisaurus, the absence of a distinct apicobasal lingual ridge might be autapomorphic for the taxon.

Klamelisaurus is similar to many other Middle-Late Jurassic Chinese sauropods in bearing denticles. Denticles are well developed on the distal and mesial margins of unworn teeth in Mamenchisaurus jingyanensis (Zhang et al. 1998), but are principally present on the mesial and apical edges of the tooth in Omeisaurus tianfuensis (He et al. 1988, figs 15-17, pl. II) and on the mesial edge in a specimen referred to Mamenchisaurus hochuanensis (Ye et al. 2001). Mamenchisaurus sinocanadorum and Mamenchisaurus youngi are likewise described as having denticles on the mesial carina of unerupted or unworn teeth (Russell \& Zheng 1993; Ouyang \& Ye 2002); however, development of denticles in these taxa is weak, and in the essentially unworn teeth of the holotype of Mamenenchisaurus sinocanadorum, denticles manifest only in the posterior-most dentary teeth as low, mesial and apical bumps (IVPP V10603 Russell \& Zheng 1993; ZDM 0083 - Ouyang \& Ye 2002, figs 8-12). The diminishment of denticles in these taxa might reflect the progressive evolutionary loss of denticles in some Core Mamenchisaurus-like Taxa. Although Bellusaurus is not recovered as a close relative of Core Mamenchisaurus-like Taxa in most of our phylogenetic analyses (see below), it is interesting that relatively posterior erupting dentary teeth bear mesial denticles while all in situ maxillary teeth (including erupting teeth) and isolated teeth have smooth carinae (Mo 2013; Moore et al. 2018).

\section{Postcranial axial skeleton}

The vertebral column of Klamelisaurus is well preserved, consisting of 21 largely complete and articulated presacral vertebrae, six sacral vertebrae and fragments of 19 caudal vertebrae from across the caudal series. Many of the caudal elements are morphologically uninformative owing to poor preservation or extensive reconstruction.

Previous authors have used various features to differentiate cervical and dorsal vertebrae in sauropods and other dinosaurs, including rib fusion (cervical ribs are fused to the diapophysis and parapophysis while dorsal ribs are free; e.g. Hatcher 1901; Gilmore 1936; McIntosh 1990; Upchurch 1998; Upchurch et al. 2004), rib angulation (cervical ribs project posteriorly while dorsal ribs project ventrally or posteroventrally; e.g. Wilson \& Upchurch 2009) and position of the parapophysis (e.g. dorsal vertebrae 
are those in which the parapophysis lies above the neurocentral suture; Sereno 1990). In Klamelisaurus, these criteria offer conflicting boundaries for the cervicodorsal transition (Supplementary Table 4), and the tenth preserved presacral vertebra (counting from anterior to posterior) exhibits both cervical and dorsal characteristics. Based on several features that vary across the cervicodorsal junction (see below), we describe the tenth preserved presacral element as the first dorsal vertebra but note that the cervicodorsal transition is not perfectly discrete. This interpretation implies nine preserved cervical vertebrae. Our interpretation of the dorsosacral junction differs from that of Zhao (1993), and we hypothesize that Klamelisaurus has 12 dorsal vertebrae and six sacral vertebrae (see below).

The continuous presacral vertebral series is broken into blocks of two to four vertebrae. It is unknown whether the break points between these blocks are due to diagenesis or reflect how the specimen was excavated. Regardless, the blocks can be readily reassembled (Supplementary Fig. 1), and the presacral series exhibits no evidence of any gaps. Zhao (1993) hypothesized that Klamelisaurus had a total of 16 cervical vertebrae. While it is not possible to predict with certainty the number of cervical vertebrae in Klamelisaurus, profiles of serial variation in several vertebral shape metrics in this taxon compare favourably with those of several other Chinese sauropods when Klamelisaurus is assumed to have had 15-17 cervical vertebrae (Supplementary Fig. 1). Nevertheless, for the purposes of our phylogenetic analysis, we scored Klamelisaurus ambiguously for cervical number. The vertebrae of IVPP V9492 are variously labelled, with articulated blocks of vertebrae given numbers corresponding to Zhao's (1993) hypothesis of 16 cervical vertebrae, while individual elements bear numbers one position more anterior. For ease of description, we refer to vertebrae by their region and number (e.g. 'cervical 9', 'sacral 4') under the assumption of 16 cervical vertebrae, though we reiterate that this number is hypothetical.

Middle to posterior cervical vertebrae. Cervical vertebrae are illustrated in figures 27 and measurements are provided in Table 1 and Supplementary Table 5.

Centra. Although the anterior condyles of the cervical centra are generally obscured by reconstruction or articulation, the exposed margins of some intercentrum articulations (e.g. between cervicals 11 and 12) indicate that the cervical centra are opisthocoelous. The steep serial decrease in centrum functional length that begins in the posterior-most cervical vertebra is preceded by an increase in the length of cervical 15 with respect to cervical 14; this pattern also occurs in the posterior cervical vertebrae of Mamenchisaurus youngi and Qijianglong (Supplementary Fig. 1). The functional elongation index (centrum functional length/average height and width of posterior articular surface; Table 1) ranges from 3.1 in cervical 9 to 1.6 in cervical 16. These values are larger overall than those in Bellusaurus (1.1-2.2; IVPP V17768), which is 
known only from disarticulated juvenile material (Dong 1990; Mo 2013; Moore et al. 2018), and closely resemble the serial trend observed in Euhelopus (Supplementary Fig. 1). On the whole, the preserved cervical vertebrae of Klamelisaurus are roughly equal in length to comparable elements in Mamenchisaurus youngi (Ouyang \& $\mathrm{He}$ 2002), longer than those in Qijianglong (QJGPM 1001) and Euhelopus (Wilson \& Upchurch 2009), and shorter than in Mamenchisaurus hochuanensis (Young \& Zhao 1972) and Omeisaurus tianfuensis (He et al. 1988).

Where exposed and unreconstructed, the ventral surfaces of the preserved centra are concave anteroposteriorly. At the level of the parapophyses, the ventral surface of the centrum is transversely concave, but posteriorly this surface develops a low, broad, transversely rounded ridge (Figs 4H, 5H). Unlike Omeisaurus tianfuensis (He et al. 1988), Omeisaurus sp. (Tan et al. 2019), Xinjiangtitan (X.-Q. Zhang et al. 2018) and Shunosaurus (Y. Zhang 1988, fig. 25), the centra lack a midline ventral keel. The posterior articular surface is broader than it is tall (cervicals 9-13) or sub-circular (cervicals 14-16). A wide or sub-circular posterior articular surface is likewise present in Cetiosaurus (Upchurch \& Martin 2002), most other Core Mamenchisaurus-like Taxa (e.g. Qijianglong - QJGPM 1001; Mamenchisaurus youngi - Ouyang \& Ye 2002), Bellusaurus (IVPP V17768) and most neosauropods, whereas the posterior cotyle is markedly taller than broad in Shunosaurus (Y. Zhang 1988), Euhelopus (Wilson \& Upchurch 2009), Mamenchisaurus hochuanensis (Young \& Zhao 1972) and Xinjiangtitan (X.-Q. Zhang et al. 2018).

Pneumatic excavation of the lateral surface of the centrum is most prominent at or just posterior to the level of the diapophysis and generally manifests as a sharp-lipped coel or coels that are crossed by an anterodorsally oblique strut (most readily apparent on the left sides of cervicals 9,12 and 13 and on the right sides of cervicals 10 and 12; Figs 2-6). These sharp-lipped excavations may be bordered by smooth, shallow concavities and low ridges, giving the adjacent bone surface an irregular texture (Fig. 2B); the lateral surface of the centrum generally loses this irregular texture as it grades posteriorly to the edge of the cotyle. It was not possible to determine whether the anterior portion of the pneumatic excavation extends onto the parapophyseal ramus of the cervical rib. The embayment of the lateral surface of the centrum can be deep, and in the sheared-off posterior half of cervical 11 the midline transverse thickness of the centrum is $23 \%$ of the width of the posterior articular surface (Fig. 4D). In the middle and posterior cervical vertebrae, the lateral pneumatic excavation occupies less than the anterior two-thirds of the length of the centrum (excluding the anterior condyle), a condition also present in some other Core Mamenchisaurus-like Taxa (Qijianglong guokr - QJGPM 1001; Mamenchisaurus sinocanadorum - IVPP V10603; Mamenchisaurus youngi - Ouyang \& Ye 2002, figs 15-17), Xinjiangtitan (X.-Q. Zhang et al. 2018), isolated cervical vertebrae from the Shishugou Formation (Wings et 
al. 2011, fig. 3; IVPP V16749, IVPP MA01), Euhelopus (Wilson \& Upchurch 2009) and some titanosauriforms (Poropat et al. 2016). An anterodorsally oblique strut is also present in Euhelopus (Wilson \& Upchurch 2009), Xinjiangtitan (X.-Q. Zhang et al. 2018), Omeisaurus junghsiensis (Young 1939, fig. 2) and most Core Mamenchisauruslike Taxa (e.g. Mamenchisaurus youngi: Ouyang \& Ye 2002, figs 15-17). In cervical 16 of Klamelisaurus, the last cervical vertebra, the septum is more dorsally positioned than in other cervical vertebrae and is orientated nearly parallel to the long axis of the centrum (Fig. 6D, E), as also appears to be the case in a cervicodorsal vertebra from the Phu Kradung Formation of Thailand (Suteethorn et al. 2013, figs 3, 4). There are no oblique septa in the lateral pneumatic fossae of any dorsal vertebrae in Klamelisaurus.

Unfortunately, reconstruction of the broken margins of the cervical centra obscures whether the internal structure of the vertebrae of Klamelisaurus was camellate, as occurs in other Core Mamenchisaurus-like Taxa (e.g. Mamenchisaurus hochuanensis Young \& Zhao 1972; Mamenchisaurus sinocanadorum - IVPP V10603, Russell \& Zheng 1993; Mamenchisaurus youngi - Ouyang \& Ye 2002). However, in other Shishugou Formation specimens with similar external pneumatic morphology (isolated cervical vertebrae - IVPP V16749, IVPP MA01; Mamenchisaurus sinocanadorum IVPP V10603), the internal structure is polycamerate or semicamellate, as confirmed by computed tomography (CT) scans of these specimens (AJM, unpublished data).

Conformation of the cervical CPRLs. The morphology of the CPRLs, TPRLs, CPOLs and TPOLs cannot be assessed except in cervicals 12 and 15. In neither element is the anterior aspect of the neural arch fully exposed. Only the existence of a stout CPRL can be determined in cervical 15, but additional details on the form of this lamina can be observed on the left side of cervical 12. In this element, an anteriorly directed fossa immediately beneath the prezygapophysis is bounded laterally by the PRDL, ventrally by a dorsoventrally expansive plate of bone and medially by a stout subvertical lamina that undergirds the ventromedial aspect of the prezygapophysis (Fig. 7A, B). Our interpretation of this unusual morphology is based on comparisons to Qijianglong and other sauropods.

Qijianglong (QJGPM 1001; Fig. 7E, F) and some other sauropods (e.g. Galeamopus pabsti - Tschopp \& Mateus 2017, figs 21, 22; juvenile Europasaurus - Carballido \& Sander 2014, fig. 6D, G; Jobaria - Fig. 7C; Bellusaurus - Fig. 7D; Daanosaurus ZDM 0193) exhibit a variant of the CPRL in which a stout lamina of the centrum intersects the PRDL laterally rather than directly contacting the prezygapophysis, producing a conjoined CPRL + PRDL dorsal to their point of intersection (Fig. 7C-F). This dorsolateral connection of the CPRL to the PRDL appears to be most common in anterior and anterior-middle cervical vertebrae. 
The cervical series of Qijianglong preserves the serial transition from a single CPRL that intercepts the PRDL to a bifurcated structure that bounds an anteriorly facing fossa, and provides the basis for our interpretation of the morphology present in cervical 12 of Klamelisaurus. Where preservation and preparation permit their morphology to be assessed, the anterior and anterior-middle cervical vertebrae of Qijianglong bear a single CPRL that intercepts the PRDL laterally, inferior to the prezygapophysis (Fig. 7E, F). In more posterior elements, pneumatic invasion of the dorsolaterally directed CPRL produces a horizontal lateral limb that meets the PRDL and a vertical medial limb that intercepts the ventromedial edge of the prezygapophysis (Fig. 7G). Some other sauropods (Daxiatitan - You et al. 2008, p. 3; cf. TendaguriaMannion et al. 2019, fig. 22A; Nigersaurus - MNN GAD 512, Fig. 7H; perhaps Australodocus - Remes 2007, fig. 3) exhibit a similar morphology, with a stout ridge of the centrum bifurcating dorsally into a subvertical CPRL that undergirds the prezygapophysis ventromedially and another limb that intercepts the PRDL laterally. These morphologies differ from the bifurcated CPRLs of most diplodocids, in which both limbs of the CPRL intercept the prezygapophysis ventrally (Fig. 7I).

The form of bifurcated CPRL observed in Qijianglong is distinct from the bifurcated CPRLs of other sauropods and notably similar to the morphology in cervical 12 of Klamelisaurus in at least two respects. First, in Klamelisaurus and Qijianglong the lateral limb of the CPRL is horizontally orientated, whereas it is distinctly dorsolaterally orientated in Nigersaurus (MNBH GAD 512; Fig. 7H) and cf. Tendaguria (Mannion et al. 2019). Thus, the lateral boundary of the fossa in Klamelisaurus and Qijianglong lacks a contribution from the lateral limb of the CPRL and is instead the continuation of the PRDL dorsal to its intersection with

the lateral limb of the CPRL, producing a fossa with parallel medial and lateral sides. Second, the horizontal lateral limb of the CPRL in Klamelisaurus and Qijianglong is dorsoventrally extensive and forms a broad anterior surface, whereas the lateral limb of the CPRL in other taxa with bifurcated CPRLs has the narrow, rounded margin that is more typical of vertebral laminae.

The anterior fossa resulting from bifurcation of the CPRL in Klamelisaurus, Qijianglong and other sauropods in which the lateral limb of the CPRL meets the PRDL does not fit perfectly into the nomenclatural scheme devised by Wilson et al. (2011), and we thus refrain from naming it. The presence or form of a medial branch of the CPRL ('mdCPRL' of Carballido \& Sander 2014) or a CPRF cannot be assessed in Klamelisaurus, as this region of the anterior surface is not visible.

Centrodiapophyseal laminae. The cervical ACDLs in Klamelisaurus are not visible and are probably obscured from view by matrix or poor preservation. The PCDL falls 
well short of reaching the posterior articular surface (Figs 3A, B, 4A-C, 5A, D, 6A, D, E). Uniquely among the cervical vertebrae, the right PCDL of cervical 12 is briefly and weakly bifurcate as it curves anteroventrally towards the diapophysis, producing a shallow longitudinal fossa, the PCDL-F (Fig. 4A, B); this feature might also be present in an isolated posterior cervical vertebra from the Flat Plain Site of the Shishugou Formation (Wings et al. 2011, fig. 3A, B). Unfortunately, the symmetry of a PCDL-F cannot be confidently assessed owing to application of plaster and paint in the corresponding region of the left side, where the diapophysis has been somewhat reconstructed; given its isolated occurrence in a single vertebra, we do not designate this feature as an autapomorphy of Klamelisaurus.

Zygapophyses, diapophyses and their connecting laminae. The sizes and orientations of the prezygapophyseal facets are generally not visible, although in cervical 12 the prezygapophysis forms a gently convex, ventromedially and anteroventrally inclined platform, the inclination of the articular surface being only slightly steeper than the transversely horizontal plane (Fig. 4D). Poor preservation obscures details of the prezygapophyseal ramus in cervicals 9 and 10, but cervicals 1116 have large, dorsoventrally tall pre-epipophyses that extend as far as (cervicals 11, 15,16 ) or beyond (cervicals 12-14) the anterior margin of the prezygapophyseal facet (Figs 4A, B, D, 5A, D, E, 6D, E). Pre-epipophyses are absent in Omeisaurus tianfuensis (He et al. 1988; ZDM T5701) and some early branching eusauropods (e.g. Shunosaurus - Y. Zhang 1988; Cetiosaurus - LEICT G468.1968) but are present in most eusauropods and well developed in Euhelopus (PMU 233), Bellusaurus (IVPP V17768) and Core Mamenchisaurus-like Taxa. A raised ridge of rugose striations likely denoting attachments of the aponeurosis transversa (1/4 septum intermusculare dorsi of crocodylians; Tsuihiji 2005) and/or the medially adjacent m. ascendens cervicalis or laterally adjacent $\mathrm{mm}$. intertransversarii (Wedel \& Sanders 2002; Tsuihiji 2005,2007 ) - is present on the lateral surface of the prezygapophyseal ramus as a posterior continuation of the pre-epipophysis (Figs 3A, B, 4A-C, 5A, D, 6A, C-E). In cervicals 10 and 11, the ridge extends in a straight line posteriorly, inferior to the SDF, to a point subequal or slightly posterior to the anterior margin of the fossa, while in cervical 12 the posterior-most extent of the ridge is slightly deflected ventrally. In cervical 13 and more posterior cervical vertebrae, the rugose ridge spans the distance between the pre-epipophysis and site of union of the PCDL and diapophysis, curving posteroventrally to a tab-like posterior projection of the diapophysis (Tschopp \& Mateus 2013; Tschopp et al. 2015a) or costal spur (Wilson \& Upchurch 2009, fig. 9; Figs $5 \mathrm{~A}, \mathrm{D}, 6 \mathrm{D}, \mathrm{E})$. The posterior projection of the diapophysis is only incipiently present in cervical 12 , but is well developed in cervicals $13-15$, jutting out posteriorly beyond the margins of the ansa costotransversaria, lateral to and separate from the PODL. This posterior projection might correspond to attachments of the $\mathrm{mm}$. 
intertransversarii (Wedel \& Sanders 2002; Tsuihiji 2005, 2007) and is well developed in various sauropods, including Spinophorosaurus ('postdiapophyseal flange' of Remes et al. 2009, fig. 3A), Shunosaurus (Y. Zhang 1988, fig. 25), Euhelopus (PMU 233; Wilson \& Upchurch 2009), Losillasaurus (MCNV Lo-1, 10-19), Mamenchisaurus hochuanensis (CCG V 20401), Camarasaurus lewisi (BYU 9047) and several diplodocoids (Tschopp et al. 2015a). In cervical 12 of Klamelisaurus, only a single raised strip of rugosities is well developed, and this is associated anteriorly with the dorsal part of the pre-epipophysis. In cervical 14, two distinct ridges are apparent, separated by a smooth groove: the first runs posteriorly from the lip of the prezygapophyseal facet, ending well anterior of the SDF, while the second, like that in cervical 12 , is born out of the pre-epipophysis and curves posteroventrally towards the costal spur (Fig. 5A). The condition in cervical 13 is obscured by damage to both prezygapophyseal rami, but it nevertheless bears a distinct, posteroventrally curving ridge, as in cervical 14. Rugose striations are abundantly present on the lateral surface of the prezygapophyseal ramus of cervical vertebrae in turiasaurians (Losillasaurus MCNV Lo-1; Turiasaurus - CPT-1220) and Jobaria (MNBH TIG 6), and a raised ridge extending posteriorly from the prezygapophysis to the SDF is well developed in Euhelopus (PMU 233; Wilson \& Upchurch 2009, figs 9, 10), Mamenchisaurus hochuanensis (CCG V 20401) and Chuanjiesaurus (LCD9701-I; Sekiya 2011, figs 9, $15)$.

Beginning in cervical 13, the lateral surface of the prezygapophyseal ramus and diapophysis ventral to the epaxial muscle attachments forms a flat, laterally and slightly dorsally facing platform that is distinctly offset posterodorsally from the remainder of the neural arch and ventrally from the lateral surface of the ansa costotransversaria (Figs 5A, D, 6A, C-E). This surface becomes more strongly developed in more posterior cervicals and in anterior dorsal vertebrae (see below), forming a distinct platform that is distinguished less by a bounding muscle scar and more by the conspicuous offset of the surface from the remainder of the neural arch, with the former facing laterally and slightly anterodorsally.

An epipophysis is present on the postzygapophyses of cervicals 8-13, but cannot be clearly assessed in more cervicals due to incomplete preservation. In 8 and 9, a low epipophysis tops the postzygapophysis and extends as far as, or slightly beyond, its articular facet. In cervicals 11 and 13, the epipophysis projects well beyond the postzygapophyseal facet (Figs 4C, 5A, D), but rather than forming an elongate, dorsoventrally flattened finger-like projection, as in Qijianglong (Xing et al. 2015, fig. $11 \mathrm{G}, \mathrm{H}$ ) and Mamenchisaurus hochuanensis (CCG V 20401; ZDM 0126), the epipophysis of Klamelisaurus is a blunt, triangular protuberance, like those in the middle cervical vertebrae of Euhelopus (Wilson \& Upchurch 2009, fig. 10) and 
Bellusaurus (IVPP V17768). A groove separates the postzygapophyseal facet from the epipophysis (Fig. 4A-C), as in Euhelopus (Wilson \& Upchurch 2009).

A raised, rugose ridge is present on the lateral surface of the postzygapophyseal ramus of the cervical vertebrae, extending anteriorly from the epipophysis beyond the level of the postzygapophyseal facet towards the SDF (Figs 4A, B, 5A-D). Whereas the epipophysis itself is probably an insertion for a tendon of $\mathrm{m}$. longus colli dorsalis and part of $\mathrm{m}$. ascendens cervicalis (Wedel \& Sanders 2002; Tsuihiji 2005), this anterior extension of the epipophysis is inferred to be a muscle scar - hereafter termed the 'anterior epipophyseal muscle scar' - that corresponds principally to the origin of mm. intercristales, with a small portion of the scar nearest the epipophysis being attributable to part of $\mathrm{m}$. ascendens cervicalis (Tsuihiji 2005; see Discussion). In cervicals 10-14, the anterior epipophyseal muscle scar juts into the SDF, producing a scalloped posterodorsal edge to the fossa (Figs 3, 4A, B, 5A-D), but this is absent in more posterior elements, where the scar meets but does not invade the posterodorsal margin of the fossa. The anterior epipophyseal muscle scar is present in some middle cervicals of Moabosaurus (Britt et al. 2017, fig. 15) and posterior cervicals of Mamenchisaurus hochuanensis (CCG V 20401), and is strongly developed in Camarasaurus lewisi (BYU 9047; 'pre-epipophyseal ridges' of Jensen 1988) and Euhelopus (PMU 233; Wilson \& Upchurch 2009, figs 9-11), where the intrusion of the scar into the SDF has been identified as part of an EPRL (Wilson \& Upchurch 2009; Wilson 2012; see Discussion). Such strong development of the anterior epipophyseal muscle scar is otherwise unknown in sauropods, although a weaker rugose line extends anteriorly from the epipophysis in some taxa (e.g. Nigersaurus - MNBH GAD 512; Kaatedocus - Tschopp \& Mateus 2013) and might be incipiently developed in Bellusaurus (IVPP V17768).

In most of the preserved cervical vertebrae, the PRDLs are markedly convex in lateral view (Figs 3A, B, 4A, B, 5A, D). Convexity of the ventral margin of the PRDL is weakly apparent in cervical 10, becomes well developed in cervical 11 through 13 , and dissipates in cervical 14 and more posterior cervical vertebrae, with the ventral margin of the PRDL approaching linearity in cervicals 15 and 16 . A ventrally convex or bulging PRDL is also present in Qijianglong (cervicals 12-14: QJGPM 1001; Xing et al. 2015, figs 11G, H, 12A-C), Euhelopus (cervicals 9-17: PMU 233; Wiman 1929, pl. III; Wilson \& Upchurch 2009, figs 9-12) and Shunosaurus (Y. Zhang 1988, fig. 26). Some middle-posterior cervical vertebrae of Bellusaurus (IVPP V17768) and Daanosaurus (ZDM 0193) develop small, tab-like processes below the preepipophyses that, while morphologically distinct from the gentle convexity of the PRDL described above, could be an ontogenetic precursor of the morphology exemplified by Klamelisaurus and Euhelopus. 
Above the diapophysis, a thick PODL extends posterodorsally to an elongate, robust postzygapophyseal ramus. On the left - but not the right - side of cervical 15 , the ventral one-third of the PODL is bifurcated, producing a tall, narrow fossa, the PODLF, between the subparallel arms of the PODL (Fig. 6A, C). A PODL-F is likewise present on the left side of cervical 16, although the more posterior arm of the bifurcated PODL is broken; the right side of C16 may bear an incipient PODL-F, but this region has been partially reconstructed. A ventrally bifurcated PODL appears to be unique to Klamelisaurus, the Phu Kradung taxon (Suteethorn et al. 2013, figs 3B, 4B), Euhelopus (PMU 233) and an articulated series of three undescribed cervicodorsal vertebrae from the Shishugou Formation at Wucaiwan in the IVPP collections (see below). A somewhat similar feature is present in the posterior cervical vertebrae of Brontosaurus parvus (UW 15556, formerly CM 563; Tschopp et al. 2015a; Gilmore 1936, pl. 31) and in the penultimate cervical vertebra of Galeamopus (Tschopp \& Mateus 2017, fig. 31) as a subvertical strut that makes up the posterior boundary of a large triangular fossa in the anterior corner of the POCDF; in the latter taxon, this strut was interpreted as a serial homologue of an isolated accessory lamina that, in more anterior vertebrae, subdivides the POCDF (Tschopp \& Mateus 2017). The morphology present in Klamelisaurus and related taxa is distinct from that in Brontosaurus parvus and Galeamopus in comprising paired laminae that diverge only slightly and in lacking the serially homologous accessory lamina of the POCDF in more anterior vertebrae (Tschopp et al. 2015a, character 199).

Neural spine. The SPRLs extend dorsally along an anteroposteriorly short neural spine. In anterior or posterior view, the lateral sides of the spine are subparallel and in cervicals 9-13 are perpendicular to a transversely flat, rugose dorsal surface. Zhao (1993) stated that the three most posterior neural spines of the cervical series were bifid. Cervicals 14 and 15 have low notches in the dorsal surface of the spine (Fig. 5F), but reconstruction in cervical 16 has obscured the extent, if any, of neural spine bifurcation. Bifurcation continues into the dorsal series, up to dorsal 5 (Supplementary Table 5; Supplementary Fig. 1D). Neural spine bifurcation is widespread in Core Mamenchisaurus-like Taxa (Mamenchisaurus youngi-Ouyang \& Ye 2002; the holotype of Mamenchisaurus hochuanensis - CCG V 20401; an isolated posterior cervical vertebra from the Phu Kradung Formation - Suteethorn et al. 2013; cervicodorsal vertebrae from the Shishugou Formation - IVPP uncatalogued specimen; Qijianglong - anterior dorsal vertebrae of QJGPM 1001), and is also present in Tienshanosaurus (Young 1937, fig. 1B) and Euhelopus (Wilson \& Upchurch 2009). The distribution of neural spine bifurcation is highly homoplastic in sauropods and is also present in some turiasaurians (Turiasaurus, Moabosaurus) and various neosauropods (Wedel \& Taylor 2013a), but is absent in Omeisaurus tianfuensis (He et al. 1988) and Bellusaurus (IVPP V17768). In the case of the latter taxon, the absence 
of neural spine bifurcation might reflect its ontogenetic stage (Woodruff \& Fowler 2012; but see Wedel \& Taylor 2013a and Melstrom et al. 2016). As in other Core Mamenchisaurus-like Taxa, the neural spine does not project far above the dorsal surface of the postzygapophyseal ramus in Klamelisaurus; in cervicals 10-14, the SPOL makes a short $(20-40 \mathrm{~mm})$, near-vertical ascent to the dorsal surface of the spine (Figs 3-5). Unlike in other cervical vertebrae, the SPOLs of cervicals 13 and 14 are weakly bifurcate just posterior to the dorsal surface of the neural spine, with a thin, anteromedially orientated lamina forming the medial boundary of a shallow, dorsally directed fossa (Fig. 5G). This feature is well developed on the left side in both cervicals 13 and 14, but on the right side only an incipient internal lamina is present in cervical 13 , and in cervical 14 this region cannot be assessed.

The PODL bounds the SDF posteriorly and is subparallel to the anterior margin of the neural spine from cervical 12 onwards. The SDF is bounded dorsally by an extensive stretch of roughly textured bone that is broadly associated with the SPOLs, SPRLs and lateral neural spine and that is offset laterally from the excavated SDF, forming an abrupt textural boundary with the smooth, pneumatized surface of the SDF. Cervicals 10-14 have irregular, sheet-like extensions of the anterolateral surface of the SPRL that begin where the anterior margin of the neural spine becomes steep, which we interpret as an autapomorphy of Klamelisaurus (Figs 3A, B, 4A-C, G, 5A-D). These sheets are rugose, becoming especially scabrous as they approach the dorsal summit of the neural spine. This collection of rugosities on the anterolateral and lateral neural spine, dorsally surrounding the SDF, might correspond to the origin of $\mathrm{m}$. longus colli dorsalis (Wedel \& Sanders 2002; Tsuihiji 2005). Similarly positioned, but morphologically distinct, extensions of the SPRL are present in some diplodocines (Tschopp et al. 2015a, character 196), including Kaatedocus siberi ('anterior bulge' of Tschopp \& Mateus 2013) and Diplodocus carngegii (Hatcher 1901, pl. III), where a dorsally restricted, tab-like projection develops in posterior cervicals, and in Erketu, where a small tubercle projects from the SPRL at mid-height in some anterior cervicals (Ksepka \& Norell 2006, fig. 6). Some middle and posterior cervical vertebrae of Bellusaurus develop transversely broad, dorsolaterally flattened SPRLs; this peculiar morphology differs from that of Klamelisaurus in lacking scabrous rugosity and an irregular anterodorsal margin and in remaining confluent with, rather than offset from, the surface of the SPRL. Nevertheless, it is possible that this morphology corresponds to nascent development of the plate-like extensions of the SPRL present in Klamelisaurus. In Klamelisaurus, long, subparallel, longitudinal striae overhang and sharply demarcate the SDF dorsally, extending from just above the anterodorsal corner of the SDF to the SPOL posteriorly. The sheet-like protrusions of the SPRLs in Klamelisaurus are less pronounced and jagged-edged in cervical 13 and more posterior 
elements, but the SDF remains clearly demarcated anterodorsally and dorsally by an expanse of muscle scars.

No distinct PRSL or POSL is apparent in any cervical vertebra, although cervical 14 bears a low, pyramidal boss in the dorsal portion of the SPRF, which is otherwise absent in the cervical series (Fig. 5F, G). The midline surfaces of the SPRF and SPOF, between the laterally bounding laminae, are low and coarsely textured. In cervical 12, the SPOF is deep anteriorly, partially undercutting the neural spine. In some cervical vertebrae, the internal surface of the postzygapophyseal ramus exhibits a series of subcircular fossae beneath the medially overhanging SPOLs. On the right side of cervical 11 , at least three fossae are preserved, which increase in diameter posteriorly (13, 18 and $23 \mathrm{~mm}$ in diameter, respectively); these are sharply excavated dorsally but are continuous with the internal surface of the ramus ventrally (Fig. 4E, F). The right side of cervical 15 bears a series of shallow, scalloped excavations with a crescentic rim delimiting their anteroventral margins (Fig. 6B). The anterior-most of these is relatively shallow and sub-circular (diameter of $18 \mathrm{~mm}$ ) and is followed posteriorly by two overlapping fossae. Far weaker scalloping is apparent in cervical 12, and might also be present in cervicals 9 and 10. In Euhelopus, only the axis exhibits fossae on the medial surfaces of its SPOLs (Wilson \& Upchurch 2009), whereas the medial surfaces of the SPOLs of more posterior cervical vertebrae are pneumatized by deep coels in Qijianglong (cervicals 14, 15 and 17: QJGPM 1001), the Phu Kradung taxon (Suteethorn et al. 2013, fig. 3C), isolated vertebrae from the Shishugou Formation (IVPP uncatalogued cervicodorsal vertebrae, IVPP V16749, IVPP MA01) and perhaps Mamenchisaurus hochuanensis (CCG V 20401). These sharp-lipped excavations presumably correspond to pneumatization by supravertebral diverticula of the supramedullary diverticulum (Wedel 2003; O’Connor 2006; Schwarz et al. 2007). Apart from these fossae, the internal, medially facing surfaces of the SPRF and SPOF are smooth in all preserved cervical vertebrae of Klamelisaurus.

Spinodiapophyseal fossa. In all cervical vertebrae with sufficient preservation (cervicals 9-15), the SDF on at least one side of the vertebra bears accessory strut(s) and subfossae inferred to result from pneumatization. This has produced a diverse array of pneumatic structures, often exhibiting substantial left-right asymmetry. The most common feature of the SDF (unambiguously present on the right in cervicals 9 and 1113, and on the left in cervicals 10 and 12-15) is a sub-horizontal strut (or paired, parallel struts: left side of cervicals 14 and 15) that divides the SDF into dorsal and ventral subfossae (Figs 4A-C, 5A-D, 6C). These struts are not laterally confluent with the PODL or SPRL (although the struts on both sides of cervical 12 approach the SPRL), and in no instance does the anterior epipophyseal muscle scar of the postzygapophyseal ramus reach the horizontal accessory strut(s) of the SDF (where present), being separated from the muscle scar by a substantial stretch of smoothly 
textured bone within the SDF, anteromedial to the PODL. Thus, in Klamelisaurus, pneumatic struts of the SDF are unmoored from all of the major processes of the vertebrae. In addition to a single, horizontal accessory strut of the SDF, the middle and posterior cervicals can exhibit additional laminae and subfossae within the SDF, which may show asymmetry within an element (e.g. cervicals 14 and 15; Fig. 5A, D, 6) (see Discussion for consideration of the homologies of laminar structures of the SDF across sauropods).

In cervicals $12-14$, just anteroventral to the SDF on the right, but not the left, side, is a deep, conspicuous, roughly triangular to ovoid blind pneumatic foramen (Figs 4A, B, 5D). The foramen is largest in cervical 12, becoming less conspicuous in more posterior elements. This feature is reminiscent of a similar foramen in some diplodocids (Tschopp et al. 2015a, characters 165 and 204; e.g. Galeamopus - Tschopp \& Mateus 2017, figs 22, 23), although in these taxa, a dorsoventrally elongate coel is present within the embayed portion of the SDF, instead of anteroventral to it as in Klamelisaurus. Despite left-right asymmetry, we tentatively designate this feature an autapomorphy of Klamelisaurus, given its persistence across several vertebrae, the regularity of its morphology and placement between vertebrae, and the morphogenetic potential in other sauropod taxa to develop similarly positioned coels.

Like the SDF, the POCDF also exhibits accessory pneumatic structures. The sharp posterior edge of the PODL overhangs the deeply excavated anterior portion of the POCDF in cervicals $12-16$. The left POCDF of cervical 13 is shallowly depressed by a weak fossa, while the right POCDF of both cervical 13 and 14 has a sharp, pronounced ventral lip below a generally irregular-textured fossa (Fig. 5E).

Cervical ribs. The cervical ribs are poorly preserved and much obscured by reconstruction. The anterior process of the right cervical rib of cervical 15 is wholly fabricated, and is missing from plate I of Zhao (1993), whereas the left cervical rib is partly reconstructed and its articulation with the centrum posteriorly appears to be fabricated (Fig. 6). The left ansa costotransversaria of cervical 16 has been broken just ventral to the diapophysis and rotated clockwise more than 90 , such that the anterior process of the rib is pointed dorsomedially, under the left PRDL; the posterior portion of the cervical rib has been pressed upwards, under the diapophysis, obscuring the lateral pneumatic fossa of the centrum. Where the ansa costotransversaria is reasonably well preserved, the angle between the capitulum and tuberculum is $<90$ and the rib shaft lies well below the ventral margin of the centrum, although not so far as in Euhelopus, in which the ribs are displaced ventrally at least as far as the height of the centrum. 
Dorsal vertebrae. The dorsal series (Figs 8-13; Supplementary Fig. 1; Table 2; Supplementary Table 5) is generally well preserved and undistorted, although portions of the neural spines, centra and diapophyses of most elements have been reconstructed.

The dorsal centra are opisthocoelous, although the extent of the anterior convexity is generally obscured by articulation. The anterior articular surface of dorsal 6 is exposed and is strongly opisthocoelous (Fig. 10A-D), and opisthocoely is retained to sacral 1 (Fig. 12H). A strongly convex condyle in the middle and posterior dorsal vertebrae is common in Core Mamenchisaurus-like Taxa (e.g. Chuanjiesaurus - Sekiya 2011, figs 16-18; Mamenchisaurus youngi - Ouyang \& Ye 2002, figs 25-27), Bellusaurus (IVPP V17768) and most macronarians. The ventral margins of the centra are markedly concave anteroposteriorly in lateral view. On the ventral surface of dorsals 1 and 2, a low ridge extends posteriorly on the midline from a point medial to the parapophyses, dissipating at approximately mid-length, where the ventral surface becomes transversely convex. In dorsals 3-7, the ventral keel extends to the ventral rim of the posterior articular cotyle (Fig. 9E); a ventral keel is absent in dorsals 8 and 12, but the condition is unknown in dorsals 9-11 due to reconstruction of the centrum.

Anterior dorsal vertebrae (dorsals 1-4). The cervicodorsal transition is not perfectly discrete. The vertebra identified here as the first dorsal bears features of both cervical and dorsal vertebrae. As in more anterior vertebrae, the parapophysis of dorsal 1 is positioned ventrolaterally on the centrum, below the lateral pneumatic fossa, and there is no SPDL on the neural arch, the SDF instead retaining the sub-horizontal accessory strut of the cervical series (Fig. 8A, B). Dorsal 1 shares with unambiguously dorsal vertebrae unfused ribs, the lack of an oblique septum in the lateral pneumatic fossa and an overall more upright neural arch. The total element height:centrum functional length ratio - a measure of the uprightness of the vertebra - increases dramatically in dorsal 1, the proportional increase being $27-152 \%$ greater than the same ratio between more anterior, adjacent elements (Supplementary Fig. 1B, D). This large disparity in total height:centrum functional length ratio between adjacent vertebrae is maintained through the four anterior dorsal vertebrae and plateaus in the middle dorsal vertebrae. A similar trend is apparent in centrum functional length (Supplementary Fig. 1A).

Centra. Lateral pneumatic excavations of the first two dorsal centra are deep, ovoid, undivided embayments whose anterior margins extend to a level subequal with the parapophysis (Fig. 8A-D). On the left side of dorsal 1, the posterior margin of the pneumatic foramen is acute (Fig. 8C-D), a character previously recovered as synapomorphic for macronarians (e.g. Mannion et al. 2013; Gonzalez Riga et al. 2018) and also present in cervicodorsal vertebrae from the Shishugou Formation (IVPP uncatalogued specimen) and Bellusaurus (IVPP V17768). Only relatively indistinct embayments are present on the remaining anterior dorsal centra and on dorsal 5 (Fig. 
9A-D), before once again becoming unambiguously pneumatic in most middle and posterior dorsal vertebrae (Figs 10, 11; see below). This shift in the form and invasiveness of excavations of the lateral surface of the centrum could reflect a cervicodorsal pneumatic hiatus between diverticula of the cervical air sacs and lungs (Wedel 2009; but see Wedel \& Taylor 2013b); alternatively, the weak depressions of dorsals 2-5 may indeed be pneumatic, perhaps reflecting weak excavation by pulmonary protuberances of the lung (O’Connor 2006).

In dorsals 2 and 3, the parapophysis has migrated from the anteroventral edge of the centrum to roughly mid-centrum height, invading the lateral fossa anteriorly, although the fossa does not extend onto the dorsal aspect of the parapophysis. The parapophysis of dorsal 4 remains on the centrum, being somewhat more dorsally positioned than in the preceding vertebrae, but by dorsal 5 the parapophysis has moved substantially dorsally, and is almost entirely above the junction of the neural arch and centrum (Fig. 9A-D).

Neural arch ventral to diapophyses. Dorsals 1 and 3 of Klamelisaurus resemble those of Mamenchisaurus youngi (Ouyang \& Ye 2002, figs 19, 20) in possessing a very large SPRF with substantial ventral excursion (Fig. 8E, F), and, as in Mamenchisaurus youngi, the anterior dorsals of Klamelisaurus appear to bear dorsoventrally tall CPRFs with little or no development of a midline lamina between the neural canal and TPRLs. On the left side of dorsal 2 and both sides of dorsal 3 a low mound of bone is visible, corresponding to the CPOL (Fig. 8J). In dorsals 3 and 4, the CPOL is closely associated with a sagittal, mediolaterally thin flange of bone that bounds the anterodorsal margin of the intervertebral foramen along the midline and corresponds to a dorsoventrally tall and posteriorly pronounced midline lamina that might have supported a hyposphene (Fig. 9A-D). The CPRL of the anterior dorsal vertebrae is a stout, columnar buttress (Figs 8E, F, H, 9A-D). Together with the ACDL and PRDL, the CPRL bounds the PRCDF. In dorsal 1, a robust, sub-horizontal strut divides the PRCDF dorsoventrally; on the right side, the ventral subfossa is further partitioned by a low strut, orthogonal to the first strut and producing a cruciate morphology (Fig. 8H), a feature that appears to be absent on the left side, although a small additional strut could be obscured by matrix. In dorsal 2, the PRCDFs are filled largely with matrix, although the exposed margins of the lamina suggest that the accessory ridges and subfossae present in dorsal 1 are absent; the homologous fossae in dorsal 4 and 5 contain weak, accessory struts (Fig. 9A-D). As in the cervical vertebrae, the PRCDF is directed anteriorly to anterolaterally, and is not well exposed in lateral view until dorsal 4, when the diapophysis has migrated sufficiently posterodorsally to expose the fossa. The PRCDF decreases in size across the anterior dorsal vertebrae owing to the retraction of the prezygapophyses, which no longer project significantly beyond the anterior condylar rim of the centrum after dorsal 5 (Figs 9A-D, 10A-D; Supplementary Fig. 1D). 
In dorsals 1 and 2, the diapophysis is supported ventrally by a distinct ACDL (Fig. 8H) and PCDL, outlining a deep, blind CDF. As in the cervical vertebrae, the PCDL is canted strongly anteriorly in the anterior-most dorsal vertebrae, and the stout CPRLs are subparallel to the PCDLs (Fig. 8A, B). In anterior dorsals 3 and 4, both laminae become increasingly upright, and by dorsal 5 the PCDL is fully vertical (Fig. 9A-D). In dorsals 3 and 4, the ACDL descends and merges with the CPRL before descending as a single lamina towards the parapophysis.

Zygapophyses and diapophyses. In the anterior dorsal vertebrae, the prezygapophyses extend beyond the anterior edge of the centrum to meet postzygapophyses that approach the level of the posterior margin of the posterior articular cotyle. Diapophyses are best preserved in dorsal 2 and the left side of dorsal 4. Across the anterior dorsals, the diapophysis migrates posterodorsally, from a position below the level of the zygapophyses in dorsals 1 and 2 to approximately level with the zygapophyses by dorsal 4. As in the posterior cervical vertebrae, the diapophyses of the anterior dorsals have a well-developed, dorsolaterally facing platform, which is strongly offset both from the more medial portion of the process and from the articular facet of the diapophysis (Figs 8, 9). In dorsal 1, especially on the right side, a rugose edge marks the boundary between the smoothly textured medial portion of the diapophysis and the rougher, more irregularly textured surface of the platform (Fig. 8G). Between cervical 14 and the middle dorsal vertebrae, the dorsolateral platform becomes smaller and less dorsoventrally extensive and, beyond the anterior dorsals, is less markedly offset from the surrounding surfaces, although it maintains a variably developed bounding ridge medially. This bounding ridge is serially homologous to the raised ridge that was inferred to be associated with the aponeurosis transversa in the cervical vertebrae (see above). In the dorsal series, the rugose platform lateral to this ridge is the site of insertion of $\mathrm{m}$. longissimus dorsi (here we use the term applied to this muscle in lepidosaurs and crocodylians, which is homologous to the dorsal part of $\mathrm{m}$. 'iliocostalis' in birds and serially homologous to the $\mathrm{mm}$. intertransversarii of the cervical series; Organ 2006; Tsuihiji 2007; Schwarz-Wings 2009).

Neural spine. As in the cervical vertebrae, the first dorsal vertebra lacks an SPDL and instead exhibits an accessory horizontal strut. This strut is quite robust on the left side, almost completely dividing the SDF into dorsal and ventral fossae (SDF1 and SDF2 of Wilson et al. 2011), but is far more weakly developed on the right side. The left SDF of dorsal 2 is undivided, whereas the right SDF appears to contain an incipient SPDL. Both sides of dorsal 3 bear a posterodorsally directed ridge that arises near the base of the diapophysis and incompletely divides the SDF; however, this ridge is not an obvious serial homologue of the SPDL, as there is an additional, weak strut on the left side of dorsal 3 that intercepts the SPRL near the base of the neural spine. Such a morphology more closely accords with the pattern of SPDL development in the 
anterior dorsal vertebrae of non-somphospondylan sauropods (Wilson et al. 2011) and is consistent with the form of the unambiguous SPDL on both sides of dorsal 4, which intercepts the SPRL just above the dorsal height of the diapophysis, producing a small and anteriorly positioned PRSDF. SPRLs are not lost through lamina capture to the SPDLs in the anterior dorsal vertebrae, as occurs in Jobaria (Wilson 2012), although the SPRLs become low and weakly developed ventrally in dorsals 3 and 4, before becoming strongly expressed in the middle dorsal vertebrae.

In dorsals 1 and 2, as in the posterior cervical vertebrae, the SDF is bounded dorsally by an expanse of epaxial muscle scars, which extends between the dorsal portion of the SPRL and the postzygapophysis. These elements appear to lack a distinct epipophysis, as in sauropods other than Moabosaurus and Janenschia (Britt et al. 2017; Mannion et al. 2019). As the neural spine shortens anteroposteriorly along the dorsal series, this rugose arc shortens into a triangular or comma-shaped plane; by dorsal 4, this arc has pulled free from the postzygapophysis, and is undergirded by a smooth, narrow SPOL (Fig. 9A-D). Above the SPOL in dorsal 4, and in the middle and posterior dorsal vertebrae, the posteroventral portion of the rugose arc splays laterally, forming a pronounced aliform process ('metapophyseal spur' of Jensen 1988; 'triangular lateral process' of Wilson \& Upchurch 2009). Neural spine bifurcation persists through all anterior dorsal vertebrae and ends with the anterior-most middle dorsal vertebra, dorsal 5 (Supplementary Fig. 1D). The notch separating the metapophyses is gently concave and 'U'-shaped in anterior view (Fig. 9F). Dorsal 1 has a small bump on the posterior midline of the base of the bifurcation notch (Fig. 8I).

\section{Middle-posterior dorsal vertebrae (dorsals 5-12).}

Centra. In dorsals 6-11, the lateral surface of the centrum bears a posteriorly acute, almond-shaped pneumatic foramen emplaced at the ventral portion of a large, shallow, sub-triangular fossa, the dorsal apex of which extends onto the base of the neural arch (Figs 10, 11), while in dorsal 12 the fossa appears to have lost its pneumatic foramen (Fig. 12A-D). The form of pneumatic excavation present in dorsals 6-11 of Klamelisaurus is common in Core Mamenchisaurus-like Taxa (e.g. Chuanjiesaurus LCD9701-I, Sekiya 2011, figs 16, 17; Mamenchisaurus youngi- Ouyang \& Ye 2002, figs 22-25, 27, pl. IX; Qijianglong - QJGPM 1001; see below). The emplacement of the lateral pneumatic foramen within the lateral fossa is reminiscent of the morphology observed in various titanosauriforms (Mannion et al. 2013, character 145), although in Core Mamenchisaurus-like Taxa the pneumatic foramen is asymmetrically positioned in the lateral fossa, at its ventral edge. Notably, the form of the lateral pneumatic excavation of Klamelisaurus differs substantially from that in the posterior dorsal centra of Bellusaurus (IVPP V17768). In the latter, a deep, sharp-lipped pneumatic 
space extends nearly to the midline of the centrum and, in at least some posterior dorsal centra, a vertical accessory strut subdivides the lateral pneumatic foramen.

The ventral-most portions of the centra of dorsals 8-11 are partially reconstructed. Parts of the last three dorsal vertebrae and the first sacral vertebra are preserved in articulation. Unlike more anterior elements, the ventral surface of dorsal 12 is transversely flat, with squared edges where the lateral and ventral surfaces meet, presaging the flat, ventral surface of the sacral vertebral centra (Fig. 12H).

Diapophyses. In anterior and posterior view, the diapophyses project dorsolaterally. Though complicated by slight deformation, the diapophyses do not appear to project more than 20 above the horizontal in middle and posterior dorsals. Unlike the anterior dorsal vertebrae, the diapophyses of posterior dorsal vertebrae are positioned dorsal to the posterior articular cotyle and project slightly above the level of the zygapophyses.

Neural arch ventral to diapophyses. By dorsal 5 , the parapophysis has migrated dorsally to the base of the neural arch (Fig. 9A-D). Although it remains too low on the neural arch to produce an ACPL, the parapophysis has interrupted both the CPRL and the ACDL, which are replaced with PRPL and PPDL, respectively. In all succeeding vertebrae, the parapophysis is well above the neurocentral junction. Dorsal migration of the parapophysis is essentially complete by dorsal 8 , and the parapophysis descends slightly in height in dorsal 9 and more posterior elements. The parapophysis remains anterior and ventral to the diapophysis throughout the series.

Beyond dorsal 5, the parapophysis is supported ventrally by a narrow ACPL (Figs $10 \mathrm{~A}-\mathrm{D}, 11 \mathrm{~A}-\mathrm{D})$; with the prezygapophyses, the anterolaterally flaring ACPLs bound an expansive anterior fossa. The left side of dorsal 6 appears to preserve a CPRL extending ventrally from the ventromedial aspect of the prezygapophysis and bounding the hypantrum laterally. Together, the CPRL and the prezygapophysis bound an anteriorly facing CPRF, as in Mamenchisaurus youngi (Ouyang \& Ye 2002; Wilson et al. 2011). A pronounced, mediolaterally thin flange of bone projects from the posterior surface of dorsal 12 between the prezygapophyses of sacral 1, suggesting the presence of a narrow hyposphene in this element.

Unlike the anterior surfaces of the neural arch, the posterior surfaces of the middle and posterior dorsal neural arches are comparatively flat, with stout, indistinct CPOLs that make up their posterolateral margins. In dorsal 7, a very low, vertically orientated lamina descends from beneath the postzygapophysis to a distance roughly halfway to the ventral edge of the PCDL, incompletely dividing the POCDF. This structure is probably an incipient lateral branch of the CPOL (sensu Tschopp et al. 2015a, character 261). This POCDF accessory lamina is well developed in the posterior dorsal vertebrae 
of several neosauropods and close outgroups, including Losillasaurus (MCNV Lo-11), Haplocanthosaurus (Hatcher 1903, pls 1, 2), Camarasaurus supremus (also middle dorsals: Osborn \& Mook 1921, pls 50-53) and Bellusaurus (IVPP V17768). In most of these taxa, the lateral CPOL projects above the embayed posterior surface as a distinct lamina, unlike the condition in Klamelisaurus, in which the incipient lateral CPOL is a low ridge on an otherwise flat surface (Fig. 13). With the exception of dorsal 7, the POCDF of middle and posterior dorsal vertebrae is uninterrupted by laminar structures, and is only weakly developed as a fossa in posterior dorsal vertebrae (e.g. dorsals 9 and 10 ), immediately lateral to the postzygapophyses. The ventral-most remnant of a very thin midline lamina is preserved immediately dorsal to the neural canal of dorsal 8 . Although the posterior surface of dorsal 8 is incompletely preserved, the dimensions of the neural canal can be estimated at roughly $27 \mathrm{~mm}$ wide and $35 \mathrm{~mm}$ tall.

In most middle and posterior dorsals beyond dorsal 6, the PRPL is weakly developed, manifesting as a stout, rounded buttress of the prezygapophysis, lacking a laminar edge (Figs 10A-D, 11A-D). In dorsal 11, this broadly rounded surface develops a peaked ridge that extends vertically and curves somewhat posteriorly, towards the PRDL (Fig. $11 \mathrm{~A}-\mathrm{D}$ ), and in dorsal 12 the PRPL more closely resembles that of the anterior dorsals, being rounded and laminar in form, and separating the PRPADF and PACPRF (Fig. 12A-D). Like the PRPL, the PPDL is also much reduced in dorsal 6, where it has been shifted dorsally and anteriorly by the migration of the parapophysis and manifests as a pinched edge above the parapophysis that meets the PRDL anteriorly, near its union with the prezygapophysis. At this junction, there are additional, short ridges that intersect the PRDL and that may be serially homologous to the accessory ridge of the PRCDF of more anterior vertebrae (Fig. 10A, B). On the left side of dorsal 7, the PPDL intercepts the base of the PRDL, while in dorsals 9-11, an incipient PPDL extends only partway to the diapophysis, such that the PRPADF and PACDF remain confluent (Fig. 11A-D). In dorsal 12, the PPDL closely approaches the diapophysis and essentially divides the PRPADF from the CDF (Fig. 12A-D; see below for description of PCPL).

In middle dorsals 5-8, the CDF is deepest dorsally, beneath the diapophysis, and grades onto the lateral surface of the neural arch, becoming essentially flat at neural arch midheight (Fig. 10A-D). Although these CDFs contain some matrix, it appears that they were not divided by accessory ridges. The CDF is bounded posteriorly by stout PCDLs that dissipate into the lateral surface of the neural arch roughly halfway down the arch. In dorsal 5, the dorsal third of the left PCDL is bifurcated, producing a narrow, elongate fossa, a PCDL-F, within the lamina (Fig. 9A, B, E); the symmetry of this feature cannot be assessed, as the right PCDL is incompletely preserved. A similarly developed PCDL-F is also present in Saltasaurus (Zurriaguz \& Powell 2015, figs 10, 11) and Bellusaurus (IVPP V17768 03-95). 
An incipient PCPL is developed on the left side of dorsal 8, and in dorsals 9-11 PCPLs are distinctly developed on the lateral surface of the neural arch (Fig. 11A-D), and divide the CDF into a shallow, indistinct CPAF ventrally and a comparatively deep PACDF dorsally (Wilson et al. 2011). There is substantial serial variation and left-right asymmetry in the form of the PCPL across these vertebrae. In dorsal 9, the left side has a stout PCPL bounding the PACDF and an additional, broad lamina ventral to it, producing a low PCPL-F; the right side also has a stout PCPL below the PACDF, with weak development of a second, and possibly an incipient third, parallel lamina beneath it. The left side of dorsal 10 has a single, thick PCPL, while the right side exhibits a triplicated PCPL, with a robust dorsal lamina subparallel to two additional, thin laminae. The right side of dorsal 11 also shows three low, subparallel laminae, while the left side more closely resembles dorsal 9 in having two subparallel PCPLs, the more dorsal of which is stouter and shorter than the ventral lamina. In these elements, the PCDL extends somewhat anteroventrally and may merge with the posterodorsal edge of the PCPL, producing a continuously rimmed ventral margin of the PACDF (Fig. 11A-D). Dorsal 12 and the sacral vertebrae lack a PCPL. A single PCPL is present in the middle-posterior dorsal vertebrae of Mamenchisaurus youngi (Ouyang $\&$ Ye 2002, fig. 24A), turiasaurians and various neosauropods (Mannion et al. 2013; Gonzalez Riga et al. 2018), but is absent in Bellusaurus, with the possible exception of an incipient lamina in one or two posterior dorsal vertebrae (IVPP V17768). Double PCPLs are also present in the Rutland specimen of Cetiosaurus (right side of dorsal 10, LEICT G468.1968; contra Upchurch \& Martin 2003), and are otherwise present homoplastically in various neosauropod taxa (Mannion et al. 2013; Tschopp et al. 2015a; Gonzalez Riga et al. 2018). Despite asymmetry and serial variability in the development of the PCPL, we consider the morphogenetic potential to produce up to three subparallel PCPLs to be an autapomorphy of Klamelisaurus.

Zygapophyses and neural spine. Where it can be observed, the zygapophyseal articulation of middle and posterior dorsal vertebrae is sub-horizontal or shallowly inclined ventromedially. By dorsal 5, the zygapophyseal articulation is above the junction between the centra and, more posteriorly, this articulation shifts into a position almost entirely posterior to the anterior condylar rim of the more posterior of the articulating vertebrae (Supplementary Fig. 1D). In dorsal 6 and more posterior elements, this shift is accompanied by posterior displacement of the neural spine, such that the anterior surface of the spine is posterior to the posterior surface of the neural arch (Figs 10-12; see below). This morphology is common in Core Mamenchisauruslike Taxa (Chuanjiesaurus - LCD9701-I, Sekiya 2011, figs 16, 17; Mamenchisaurus hochuanensis - CCG V 20401, ZDM 0126; Mamenchisaurus youngi-Ouyang \& Ye 2002, pl. IX) and is also present in Dongbeititan (X. Wang et al. 2007, fig. 1). In middle and posterior dorsal vertebrae, the neural spine has subparallel lateral margins 
above the lateral flaring of the aliform processes and is tall and vertically straight or angled slightly anteriorly. At its apex, the neural spine of dorsal 12 preserves a subtriangular posterior projection (Fig. 12A-D, F); it is possible that this projection corresponds to metaplastic mineralization of a portion of the supraspinous or interspinous ligament (Woodruff 2014, 2017; Cerda et al. 2015; Horner et al. 2016), although this remains to be tested histologically.

SPRLs are present across the entire dorsal series, in contrast to some sauropod taxa in which the SPRLs become stranded spinal laminae (e.g. Tazoudasaurus: Allain \& Aquesbi 2008, fig. 14) or are lost via lamina capture to become SPDLs (e.g. JobariaWilson 2012; Camarasaurus supremus - Osborn \& Mook 1921, pls 70-73). In Klamelisaurus, the SPRLs are well-developed struts that bound the SDF anteriorly and the SPRF laterally, and remain roughly subparallel throughout their extent along the anterolateral edges of the neural spine. In dorsal 6 and more posterior elements, the SPRLs no longer reach the dorsal height of the neural spine, producing a triangular, plate-like buttress, the posterodorsal corner of which intersects the body of the spine at progressively lower levels, ascending less than half the height of the neural spine in the posterior-most dorsal vertebrae (Fig. 12A-D). Where the dorsal margin of the SPRL meets the anterior face of the neural spine, the lamina dissipates, becoming nearly indistinct from the rugose infilling of the SPRF. In all middle to posterior dorsal vertebrae, the SPRF lacks a distinct PRSL, and instead bears a low, rough infilling of the SPRF above the height of the prezygapophyses.

In middle and posterior dorsal vertebrae, an incipient SPDL generally falls short of reaching either the SPRL or SPOL, producing an incompletely divided SDF. The SDFs of dorsal 5 are only partially preserved, but it is clear that the SPDL was directed towards the SPRLs. The right SPDL of dorsal 6 incompletely divides the SDF, extending into the fossa at an angle subparallel to the SPRL and falling short of reaching the SPOL, while on the left side, an essentially vertical SPDL extends from the posteromedial edge of the diapophysis to the SPRL, although the robusticity of this lamina is likely exaggerated by substantial reconstruction. The SPDL is a single, stout lamina in the ventral half of the left SDF of dorsal 7, while on the right side of this element, a robust SPDL bifurcates dorsally into two branches that bound a large, deep pneumatic recess (Figs 10G, 13A). The form of the SPDL is ambiguous or poorly preserved in dorsals 8 and 11 but it is clearly bifurcated dorsally in dorsals 9 and 10 . In dorsal 12 and sacral 1, the SPDL is a single lamina that extends a short distance up the neural spine towards the SPOL, incompletely dividing the SDF into a large PRSDF and small POSDF. A dorsally bifurcate SPDL in middle and posterior dorsal vertebrae was identified as an autapomorphy of Bellusaurus by Mo (2013), although in the latter the anterior and posterior branches extend entirely to the SPRL and SPOL, respectively, in at least some elements (IVPP V17768). A bifurcate SPDL is relatively uncommon 
among sauropods, and is otherwise present only in the turiasaurians Mierasaurus and Moabosaurus and in some titanosaurians (Poropat et al. 2016; Mannion et al. 2019). We interpret a dorsally bifurcated SPDL in middle and posterior dorsal vertebrae without confluence with either the SPRL or the SPOL to be an autapomorphy of Klamelisaurus.

The SPOLs of the middle and posterior dorsal vertebrae are unbifurcated ventrally and extend farther laterally than the SPRLs, owing to the flaring aliform processes. The aliform processes have greater lateral excursion than the outer edges of the postzygapophyseal facets. In the posterior-most dorsal vertebrae, the SPOLs extend laterally and only slightly posteriorly (Fig. 12A-D). From the dorsal extent of the neural spine to a level just below mid-height of the neural spine, the midline rugosities in the SPOF of middle and posterior dorsal vertebrae become transversely rounded, extending posteriorly from the SPOF as a low, broad, rugose POSL. The interior faces of the SPOLs are smooth ventrally but become rugose dorsally as they approach the aliform processes. Above the aliform processes externally is a rugose, ' $\mathrm{J}$ '-shaped or sub-triangular platform that projects dorsolaterally and is confluent dorsally with the summit of the neural spine. In the posterior dorsal vertebrae, the posteroventral projection of the distal corner of the aliform process suggests that the process was connected to the lateral anterodorsal corner of the diapophysis of the succeeding vertebra by epaxial musculature or a ligament. This inference is bolstered by the presence of an anterolaterally positioned spur ('prediapophyseal spur' of Jensen 1988) on the left transverse process of dorsal 12 and sacral 1 that points towards the preceding aliform process (Fig. 12A-D, F) and by the presence of mineralized ligaments linking these structures in the sacrum of Camarasaurus lewisi (BYU 9047; Jensen 1988; McIntosh et al. 1996; see below).

Dorsal ribs. Zhao (1993) noted that few dorsal ribs are preserved and listed the dimensions of the ribs for dorsal vertebrae 3, 4 and 6 (Supplementary Table 6). We were able to locate only one of these, a right dorsal rib labelled as that of dorsal 4 (Fig. 14A). Only the proximal portion of this rib (approximately $290 \mathrm{~mm}$ ) is unreconstructed. We describe the rib as orientated in a transverse plane.

The capitulum is dorsoventrally elongate and in proximal view consists of an anteroposteriorly broad ventral head connected to a smaller dorsal head by a thin lamina of bone. The articular surface of the tuberculum is roughly sub-triangular in proximal view, with anteroventral, anterodorsal and posterior margins. The anteroventral margin is continuous with a fossa on the anterior surface of the thin plate of bone that stretches between the capitulum and tuberculum. A sharp ridge extends laterally from the junction of the anteroventral and anterodorsal surfaces of the tuberculum, extending along the anterior surface of the rib. The breadth of the 
anterodorsal surface of the rib increases distally. The proximal shaft of the rib is roughly sub-triangular in cross section and the posterior surface of the shaft is concave.

The fossa on the proximal anterior surface of the rib, between the two articular heads, is bounded distally by a partially reconstructed ridge, the orientation of which suggests that it reinforces the capitulum (Fig. 14A). Proximal to this, a broader, shorter, subparallel ridge is present within the fossa, just ventral to the sharp anterior ridge of the tuberculum. Similar dual ridges are present on the proximal anterior surface of an anterior dorsal rib of Bellusaurus (IVPP V17768.212; Fig. 14B). The distribution of these ridges among sauropods is unclear, but a ridge that reinforces the capitulum (which we infer to be homologous to the more distal of the two ridges in Klamelisaurus and Bellusaurus) is widespread in sauropods, including Mamenchisaurus hochuanensis (Young \& Zhao 1972, pl. 4), Qijianglong (QJGPM 1001), Haplocanthosaurus (Hatcher 1903, fig. 12), Camarasaurus (Osborn \& Mook 1921, pls 78, 81) and Apatosaurus (Gilmore 1936, pl. 29).

Sacral vertebrae. Our interpretation of the number of dorsal and sacral vertebrae in Klamelisaurus differs from that of Zhao (1993). We interpret Klamelisaurus as probably having an additional dorsosacral vertebra, with a total of six sacral vertebrae. Because the left ilium is missing and the right ilium is separate from the axial skeleton, the standard criterion for identifying sacral vertebrae - contact with the ilium - cannot be assessed. Another feature that typically characterizes sacral vertebrae in skeletally mature sauropods - coossification of adjacent vertebral centra - cannot be confidently determined for precaudal 23 (dorsal 13 of Zhao 1993; sacral 1 of the present study). Our hypothesis that precaudal 23 is the first sacral vertebra is based on the presence of a bridge of bone connecting the diapophysis and parapophysis on both sides of the element (Fig. 12A-D, E). This bridge forms the lateral margin of a transverse foramen lacking open costovertebral junctions. One interpretation for this structure is that it comprises the medial portion of the rib that encloses the transverse foramen laterally. Although the ribs of posterior dorsal vertebrae occasionally co-ossify with the diapophysis and parapophysis, such co-ossification is characteristic of sacral vertebrae in sauropods (Wilson 2011). This interpretation implies that the majority of the rib has been lost to weathering. An alternative explanation is that the rib is missing and this bar of bone instead represents incipient stages of the coalescence of the diapophysis and parapophysis that occurs over the course of the sacral series and that accounts for the loss of the transverse foramen in more posterior sacral vertebrae and caudal vertebrae (Wilson 2011). Such coalescence of the diapophyseal and parapophyseal components of the costovertebral junction is present in the succeeding vertebra, which lacks a transverse foramen and exhibits a vertically elongate articular surface that extends from the neural arch to the ventral half of the centrum. Although we refer to precaudal 23 as 
'sacral 1' for the remainder of the description, future discoveries are required to confirm that Klamelisaurus has six vertebrae that articulate with the ilium.

In most other respects, sacral 1 resembles the posterior-most dorsal vertebrae. Like dorsal 12, sacral 1 has a well-developed PRPL, as well as a triangular recess on its centrum that appears to lack a pneumatic foramen (Fig. 12A-D). In more posterior sacral vertebrae, there is no evidence of pneumatic excavation of the lateral surface of the centrum, although preservation in sacrals $3-6$ is poor, and none retain unweathered bone on their lateral surfaces (Fig. 15A, B). Poor preservation obscures the boundaries between sacral elements and the shape of the posterior surface of sacral 6. Nonetheless, it is apparent that the functional centrum length of the sacral vertebrae is much diminished with respect to the centra of posterior dorsal vertebrae, especially between sacrals 3 and 6 , where the average centrum length (c. $118 \mathrm{~mm}$ ) is $64-80 \%$ the length of the posterior dorsal vertebrae (Table 3 ). The ventral surfaces of the sacral vertebrae lack a midline keel; this surface is transversely concave in sacral 1 (Fig. 12H), nearly flat in sacrals 2-5 and markedly concave in sacral 6, although preservation of the latter is poor. The diapophyseal and parapophyseal projections of sacrals 3-6 are poorly preserved, but likely resembled the condition in sacral 2 in forming a dorsoventrally continuous process that articulated with the rib.

The neural spines of sacrals $2-5$ are co-ossified throughout their height, forming a continuous plate dorsally (Fig. 15). Fusion of at least four anterior sacral neural spines is also present in Mamenchisaurus youngi (Ouyang \& Ye 2002, fig. 29) and Bellusaurus (IVPP V17768.109), but is otherwise restricted to Tastavinsaurus and more derived titanosauriforms (Mannion et al. 2013, character 174). The neural spine of sacral 6 is separate from that of sacral 5 and has been reconstructed dorsally; its neural spine is very slightly canted posteriorly with respect to more anterior elements, but is still essentially vertical, unlike the posteriorly deflected spines of the anterior caudal vertebrae (see below). The dorsal surfaces of the neural spines are rugose and pitted, but there is no distinct, rod-like cap to the spines that would indicate the presence of a metaplastically mineralized supraspinous ligament, which is present in Epachthosaurus, Malawisaurus and several other titanosauriforms (Cerda et al. 2015; see also Woodruff et al. 2016). The aliform processes become especially drawn out and elongate in the sacral vertebrae, and extend well below midheight of the neural spine. A narrow buttress connects the left aliform process of sacral 1 with the anterolateral corner of the diapophysis of sacral 2 (Fig. 12A, B). No original bone is readily apparent in this buttress, and its surface appears to be composed entirely of reconstructive material. Nevertheless, as noted for the posterior dorsal and sacral vertebrae, the possible presence of a soft tissue structure linking the aliform process and diapophysis of adjacent vertebrae is consistent with the orientation of the bony 
projections of these processes and the preservation of identically situated mineralized ligaments in Camarasaurus lewisi (BYU 9047; Jensen 1988; McIntosh et al. 1996).

The homologies of the laminae associated with the fused neural spines are difficult to discern owing to their coalescence, but unlike more anterior vertebrae the left and right sides are highly symmetrical (Fig. 15A, B). The SPDL is identified by its association with the diapophysis. On both sides, a triangular fossa is present ventral and slightly anterior to the aliform process of sacral 3 . The anterodorsal edge of this fossa is composed of a lamina extending posterodorsally from the diapophysis of sacral 3 perhaps a continuation of the SPDL of the latter - while its posterodorsal edge is composed of a novel lamina extending from the SPDL of sacral 4; at their dorsal union, these two laminae form a composite lamina that supports the aliform process of sacral 3 ventrally. The ventral edge of the triangular fossa is formed by a lamina that spans the space between the SPDLs of sacrals 3 and 4 . The lamina supporting the aliform process of sacral 4 is especially stout, and is ventrally the product of the union of an SPDL extending anteromedially from the diapophysis of sacral 5 and a thick, short branch extending posteriorly from the diapophysis of sacral 4 . An additional strut projects anterodorsally from this union, forming the ventral boundary of a distinct, oval embayment on the lateral surface of the neural spine of sacral 4. In general, the lateral surface of the neural spine of sacral 4 shows the most pneumatic sculpturing among the sacral vertebrae. A large, triangular fossa is present on the lateral surface of sacral 6 . Posteriorly, it is bounded by the stout SPDL of sacral 6; its dorsal edge is formed by a short, horizontal ridge corresponding to the prezygapophysis, while its anteroventral edge comprises a thin, elongate strut that appears to unite the diapophysis of sacral 6 with the postzygapophysis of sacral 5 .

Caudal vertebrae. Zhao (1993) stated that the holotype included two complete anterior caudal vertebrae, 10 anterior caudal neural spines and 10 middle caudal centra, from which he estimated that more than 60 caudal vertebrae would have been present originally. Among the material housed with the specimen, we located four partial anterior-most caudal vertebrae, five anterior caudal neural spines and 11 middleposterior centra (Figs 15, 16; Tables 4, 5), all of which are poorly preserved and highly reconstructed. Below, we identify caudal vertebrae by the number ascribed to them when the specimen was mounted. The relative sizes of the caudal vertebrae suggest that they are correctly ordered from anterior to posterior.

The first caudal vertebra is preserved in articulation with sacral 6 (Fig. 15); caudals 2-4 are preserved in articulation in a separate block (Fig. 16A), but the size and form of these vertebrae suggest that they form a series continuous with caudal 1. Caudal 3 is the best preserved of the anterior-most caudal vertebrae (Fig. 16A). 
There is no evidence for pneumatic embayments on the anterior-most caudal centra, although poor preservation prevents detailed description of their lateral surfaces. Transverse processes are also missing from these elements, except in caudal 4 , where the ventral portion of the transverse process is poorly preserved and morphologically uninformative. The preserved portions of the centra of caudal 3 and caudal 4 suggest that these elements are procoelous, as described by Zhao (1993), but details of their form are difficult to determine, and the large, mushroom-shaped posterior condyle of caudal 4 is mostly fabricated.

In the anterior-most caudal vertebrae, the prezygapophysis extends far anterior to the anterior articular surface, while the postzygapophysis is short and does not extend beyond the posterior margin of the centrum. The angle of zygapophyseal articulation between sacral 6 and caudal 1 is much steeper than in the dorsal series, with transverse angulation of the zygapophyseal facet exceeding 60 , as is also true in the succeeding anteriormost caudal vertebrae. The left side of caudal 3 preserves a stout PRDL that extends anterodorsally from mid-height of the neural arch to the prezygapophysis, and overhangs an anteriorly and slightly laterally facing PRCDF. Dorsal to the PRDL, a distinct PODL courses posterodorsally, dissipating near the dorsal height of the postzygapophysis. The lateral and posterior surfaces of the neural arches meet at a rounded corner, with no distinct development of a lamina. In caudal 3, a short, stout midline ridge is preserved beneath the postzygapophyses, and presumably corresponds to a hyposphenal ridge.

The neural spines of the four anterior-most caudal vertebrae are claviform in shape: they expand anteroposteriorly and transversely towards their distal ends and lack distinct edges or well-developed spinozygapophyseal laminae separating their anterior, posterior and lateral surfaces (Figs 15, 16A). A claviform neural spine on the anteriormost caudal vertebrae is likewise present in Chuanjiesaurus (LCD9701-I), the holotype, but not the referred specimen, of Mamenchisaurus hochuanensis (CCG V 20401; ZDM 0126) and Wamweracaudia keranjei (HMN MB.R.2091.1-30; Janensch 1929b; Bonaparte et al. 2000; Mannion et al. 2019), although in the latter case the SPRLs and SPOLs are more fully developed and make the margins of the spine more distinct. By contrast, Mamenchisaurus youngi (Ouyang \& Ye 2002, fig. 30) has anterior-most caudal vertebrae with neural spines that are mediolaterally compressed. Caudal 1 of Klamelisaurus is notably distinct from the sacral vertebrae in having a neural spine that is canted strongly posterodorsally (Fig. 15), a feature described by Zhao (1993) as diagnostic for the taxon. Despite reconstruction of the neural arch, we consider this deflection of the neural spine to be genuine, given similar deflections in the succeeding three vertebrae, in which the neural arches are at least partly intact (Fig. 16A). Posterior deflection of the neural spine is typical of several other Middle-Late Jurassic Chinese sauropods (e.g. Wamweracaudia - HMN MB.R.2091.1-30; 
Chuanjiesaurus - LCD9701-I; Mamenchisaurus youngi - Ouyang \& Ye 2002, fig. 31, pl. XIV; Bellusaurus - IVPP V17768; Mamenchisaurus hochuanensis - CCG V 20401). Klamelisaurus may be unique in the degree and abruptness (with respect to sacrals 1-6) of the posterior deflection of the anterior-most caudal neural spines, but we withhold designation of this feature as diagnostic of the taxon in the absence of better-preserved specimens.

Elements labelled as caudals 6 and $8-11$ preserve only their neural spines. These anterior neural spines are similar to those in the anterior-most caudal vertebrae and are claviform in shape, though somewhat more compressed mediolaterally (Fig. 16B, C). The anterior neural spines lack the marked mediolateral expansion of the distal end of the spine that is present in Camarasaurus (e.g. BYU 9047; Osborn \& Mook 1921, pls 74-77), Omeisaurus tianfuensis (ZDM T5701) and some other sauropods (Mannion et al. 2013).

The remainder of the preserved caudal vertebrae are labelled as caudals 18-27 and 33 (Fig. 16D-N). All of these lack neural spines, and in most cases the centrum is at least partly reconstructed. Given their relatively small size and lack of transverse processes, these are likely to be posterior middle and posterior caudal vertebrae. Both articular surfaces of the centra are either flat or very shallowly concave, and all of the centra lack longitudinal ridges on their lateral surfaces. The centra are waisted, with the midwidth of the centrum generally two-thirds or less the width of the posterior articular surface. The ventral surfaces of caudals 18,19 and 22-25 are transversely convex, while caudals $20,21,26,27$ and 33 have a weak sulcus or are longitudinally flat. Where they can be assessed, the centrum articular surfaces are sub-circular (caudal 22) to sub-quadrangular (caudal 26) (Fig. 16G, J, M).

Chevrons. No chevrons were mentioned by Zhao (1993); however, we found four middle or posterior chevrons in the crates containing the holotype, labelled as chevrons 26 and 28-30 (Fig. 16O-V; Table 6). All are essentially complete and have separate anterior and posterior expansions of the distal blade (Fig. 16P, R, T, V), as is plesiomorphic for eusauropods (Wilson \& Sereno 1998). The two halves of the chevron are fused distally along their length. The lateral surfaces of the chevrons are rugose throughout, but lack accessory ridges or processes. The chevrons decrease in proximodistal height and increase in distal anteroposterior length along the series. Proximally, the haemal canals of chevrons 29 and 30 are fully bridged by bone, whereas in chevrons 26 and 28 the two arms bounding the haemal canal appear to be closely appressed but unfused. The anterior and posterior surfaces of chevron 29 bear a sub-triangular fossa just below the haemal canal (Fig. 16S), as in Zby (Mateus et al. 2014). The proximal articular surface of chevron 26 is concave transversely and convex anteroposteriorly and is only weakly divided into anterior and posterior facets, 
but partitioning of the proximal surface into separate facets is more clearly developed in chevrons 28-30. In chevron 29, the proximal surface is narrower anteroposteriorly than in other chevrons, and its haemal canal is wider than in the other elements.

\section{Scapula}

We describe the scapulocoracoid with its long axis orientated horizontally (Fig. 17). The scapula and coracoid are fused with no discrete suture between them, although their junction is marked by a slight ridge. Metal armature and a painted plaster jacket cover the entire medial surfaces of both elements and were in place when the photographs in Zhao (1993, pl. I) were taken. The brief description of the scapula offered by Zhao (1993) suggested that the scapular blade is intact; however, as preserved, most of the scapular blade is covered by plaster and paint, and we were unable to confidently trace signs of original bone beyond the midpoint of the blade. That the blade has been restored substantially is borne out by the dramatic flaring of the distal end of the blade in Zhao (1993, pl. I), which differs notably from the more gently expanded distal end currently in place. Thus, we do not consider the length or shape of the scapular blade to be reliable (Table 7). The dorsal extent of the junction between the scapula and coracoid has also been partially reconstructed, although the extent of fabrication is difficult to determine and might be slight.

The acromion process is anteroposteriorly broad, unlike the condition in Cetiosaurus (OUMNH J.13606), Shunosaurus (Y. Zhang 1988, fig. 42, pl. XII) and most noneusauropod sauropodomorphs, in which the breadth of the acromion process is less than $150 \%$ the minimum width of the scapular blade (Wilson \& Sereno 1998). The acromial ridge curves posterodorsally from an area just posterior to the glenoid fossa to the posterodorsal margin of the acromion process, bounding the acromial fossa posteroventrally. The posterior margin of the acromion process is straight, unlike the concave posterior margin present in Mamenchisaurus youngi (Ouyang \& Ye 2002, fig. 33, pl. XVI), Euhelopus (Young 1935, figs 2, 3), Daxiatitan (You et al. 2008, fig. 2) and various neosauropods (Mannion 2009; Mannion et al. 2013), and the acromial ridge is orientated at an angle just less than perpendicular to the scapular blade. There is no fossa or excavation posterior to the acromial ridge, unlike the condition seen in several neosauropod taxa (Upchurch et al. 2004). The dorsal margin of the acromion process projects above the height of the acromion ridge and is convexly rounded, lacking the dorsal concavity of rebbachisaurids (Mannion 2009).

In cross section, the scapular blade appears to be ' $\mathrm{D}$ '-shaped, as in most nonsomphospondylan eusauropods (Wilson 2002). Unfortunately, the ventral margin of the scapular blade is highly reconstructed, and the presence of a sub-triangular process cannot be confidently assessed; such a process is present in other Core 
Mamenchisaurus-like Taxa (Mamenchisaurus youngi - Ouyang \& Ye 2002, fig. 33, pl. XVI; Chuanjiesaurus - Sekiya 2011, figs 36, 37; perhaps the referred specimen of Mamenchisaurus hochuanensis - Ye et al. 2001, pl. I), Tienshanosaurus (IVPP RV37089; Young 1937, fig. 5), Euhelopus (Young 1935, figs 2, 3), Omeisaurus tianfuensis (ZDM T5701; He et al. 1988, pl. XIII), Bellusaurus (IVPP V17768) and some titanosauriforms (Mannion et al. 2013, character 216). The presence of a similar but distinct sub-triangular process on the posteroventral corner of the scapular acromion cannot be confidently assessed owing to reconstruction, but this feature is absent in most non-somphospondylan sauropods (D'Emic et al. 2011; Mannion et al. 2013).

The scapular portion of the glenoid is essentially flat, angled anteroventrally and bevelled somewhat medially with respect to the coracoid portion of the glenoid and the long axis of the scapula, such that the scapular and coracoid glenoid facets are offset with respect to each other (Fig. 17B). Medial deflection of the scapular glenoid is also present in Jobaria (MNBH TIG 4), Zby (Mateus et al. 2014), Euhelopus (Young 1935, fig. 2; Wilson \& Sereno 1998), Apatosaurus (Wilson 2002) and most titanosauriforms (Wilson \& Sereno 1998; Mannion et al. 2013).

\section{Coracoid}

The maximum anteroposterior length of the coracoid is subequal to its dorsoventral height (Table 7). In lateral view its shape is slightly trapezoidal, as noted by Zhao (1993), with a longer dorsal than ventral margin (Fig. 17). As in other non-titanosaurian sauropods, the dorsal margin of the coracoid lies below the level of the acromion process. The anterodorsal corner of the coracoid is squared as in Chuanjiesaurus (Sekiya 2011, figs 36, 37) and some titanosauriforms, but unlike Bellusaurus (IVPP V17768) and most sauropods. A sub-circular, matrix-filled coracoid foramen is well separated from the scapula-coracoid articulation. The medial surface of the coracoid is concave while its lateral surface is gently convex anteroposteriorly.

Much of the external surface of the coracoid is rugose, especially in its ventral half. The distinct biceps tubercle present in Spinophorosaurus (Remes et al. 2009) is absent in Klamelisaurus. The angle between the scapula and coracoid portions of the glenoid is slightly greater than 90 . The coracoid portion of the glenoid is gently convex transversely, with slightly less than two-thirds of the surface projecting posteroventrally and the remainder of the glenoid wrapping slightly onto the lateral face of the coracoid, producing two weakly distinguishable surfaces, as in other sauropods (D'Emic \& Foreman 2012). A broad, rugose, laterally projecting ridge is present at the juncture of the glenoid and the lateral face of the coracoid. The ventral edge of the coracoid anterior to the glenoid is weakly concave, as in Mamenchisaurus 
youngi (Ouyang \& Ye 2002, fig. 33, pl. XVI), Chuanjiesaurus (Sekiya 2011, fig. 37) and various other sauropods, but unlike the comparatively deep infraglenoid groove, or notch, present in Tienshanosaurus (IVPP RV37089) and Bellusaurus (IVPP V17768). Unlike Tehuelchesaurus (Carballido et al. 2011, fig. 14), there is no well-developed, pendant infraglenoid lip anterior to the infraglenoid groove.

\section{Humerus}

The right humerus is essentially complete and undistorted, save for light reconstruction of the distal articular surface and inward crushing of part of the posterior surface of the distal humerus, proximal to the lateral condyle (Fig. 18; Table 8). Metal armature extends along the length of the element on its posterior side.

The proximal articular surface is rugose and pitted. In anterior and posterior view, this surface is strongly and nearly symmetrically convex dorsally, and the humerus lacks the squared-off proximolateral corner present in Tehuelchesaurus (Carballido et al. 2011, fig. 15A), Haestasaurus (Upchurch et al. 2015, fig. 2), Euhelopus (Young 1935, fig. 5), rebbachisaurids (Carballido et al. 2012) and somphospondylan titanosauriforms (Upchurch 1999; Wilson 2002; Mannion et al. 2013). The dorsal peak of the proximal articular surface is approximately even with the lateral-most excursion of the curving medial edge of the humeral shaft. The anteroposteriorly widest part of the proximal end is located medial to the median longitudinal axis of the humerus (Fig. 18A, B). Unlike Bellusaurus (IVPP V17768), Euhelopus (Young 1935; Poropat et al. 2016), Cetiosauriscus (NHMUK PV R3078) and most neosauropods (Upchurch et al. 2015; Poropat et al. 2016), the humeral head does not form a sub-circular process that overhangs the posterior surface of the proximal end, and the proximal half of the posterior surface of the humerus is only weakly concave in lateral and medial views. Nevertheless, the gentle posterior expansion of the humeral head gives rise to a broad, low ridge that weakly separates the posterior surface into shallow medial and lateral fossae (Fig. 18D). Some previous authors have interpreted these fossae as housing the origins of medial and lateral portions of the triceps muscle (Borsuk-Białynicka 1977; Upchurch et al. 2015), but they might instead correspond to insertions for $\mathrm{Mm}$. scapulohumerales, with the humeral heads of the triceps musculature originating farther distally (Remes 2008; Otero 2018). Where it is not obscured by armature, the posteromedial edge of the proximal end bears longitudinal, parallel striations, which are especially prominent near the medial margin (Fig. 18D).

The proximolateral corner is less strongly expanded than the proximomedial corner, such that the medial margin of the humerus is more concave than the lateral margin. The asymmetry in degree of proximal flaring of the humerus in Klamelisaurus is distinct from the marked asymmetry of some turiasaurians (Losillasaurus - MCNV 
Lo-7; Turiasaurus - CPT 1195) and somphospondylans (Curry Rogers 2005; Mannion et al. 2013), where the entirety of the lateral margin of the humerus is essentially straight. Depression of the anterior aspect of the proximomedial corner of the humerus produces a shelf of bone, $170 \mathrm{~mm}$ in length, that extends transversely across nearly half of the anterior surface of the proximal humerus. The surface of this shelf is strongly sigmoid in anterior view, such that it is bounded medially by a low, rounded bump (Fig. $18 \mathrm{~A}, \mathrm{~B}, \mathrm{~F})$. This shelf-like depression of the proximomedial corner of the anterior surface is autapomorphic for Klamelisaurus.

The anterior surface of the proximal half of the humerus is mediolaterally concave. The surface of the bone has been fractured and the ventral portion of the anterior fossa has been depressed, making the anterior fossa appear deeper than it was in life, especially distally. A low, sub-circular rugosity is weakly expressed near the centre of the anterior concavity, presumably corresponding to insertions of $\mathrm{m}$. coracobrachialis (BorsukBiałynicka 1977; Remes 2008; Otero 2018). This scar is present in Janenschia (Mannion et al. 2019) and most neosauropods (Upchurch et al. 2015) and is weakly developed in Cetiosaurus (OUMNH J.13612), but is absent in Bellusaurus (IVPP V17768), although this might be due to the young ontogenetic stage of the known specimens. In Klamelisaurus, longitudinal striations are present at the proximomedial corner of the anterior surface, just distal to the autapomorphic proximomedial shelf.

With the distal articular surface orientated transversely, the deltopectoral crest projects anteriorly and very slightly medially. Where it arises proximally, the deltopectoral crest is relatively thin, but it doubles in mediolateral thickness distally. Distal widening of the deltopectoral crest is absent in other Core Mamenchisaurus-like Taxa (Mamenchisaurus youngi - Ouyang \& Ye 2002, fig. 35; Chuanjiesaurus - figs 38, 39) and Bellusaurus (IVPP V17768), but is present in Cetiosaurus (OUMNH J.13612), Turiasaurus (CPT 1195) and some titanosaurians (Poropat et al. 2016). The deltopectoral crest projects most prominently at approximately $38 \%$ of the distance from the proximal articular surface. At its minimum transverse width, the humeral shaft is $60 \%$ wider transversely than anteroposteriorly, exhibiting a degree of eccentricity that is typical of non-neosauropods other than Jobaria and Lapparentosaurus (Mannion et al. 2017), which have more circular cross sections. The humeral shaft is twisted, such that the long axes of the proximal and distal articular surfaces are not parallel. With the distal articular surface orientated transversely, the long axis of the proximal articular surface is canted anterolaterally, such that the proximal fossa bounded laterally by the deltopectoral crest faces somewhat anteromedially. The angle between these long axes is difficult to measure, but is at least $30^{\circ}$.

A pronounced, sub-circular, boss-like muscle scar projects posteriorly from the ventrolateral edge of the lateral, proximal fossa at a level above the most prominent 
point of the deltopectoral crest (Fig. 18G). Remes (2008, p. 279) observed that in Klamelisaurus and other Core Mamenchisaurus-like Taxa, this scar is in a similar position to the $\mathrm{m}$. latissimus dorsi scar of many non-sauropod sauropodomorphs, but is considerably larger, and noted that this scar might alternatively correspond to a distally shifted insertion of $\mathrm{m}$. deltoideus scapularis, or a specialized part of $\mathrm{m}$. deltoideus clavicularis. Based on muscle reconstructions by Borsuk-Białynicka (1977), this scar has also been interpreted as the insertion for the $\mathrm{m}$. scapulohumeralis anterior (Upchurch et al. 2015). In Cetiosauriscus (NHMUK PV R3078), Jobaria (Poropat et al. 2016) and several titanosauriforms (Upchurch et al. 2015; Poropat et al. 2016), this scar bulges laterally, and is visible in anterior view; in Klamelisaurus, a posterolateral scar is just barely perceptible as a low interruption in an otherwise essentially linear profile (Fig. 18D). Remes (2008) recognized only a single posterolateral tubercle in Klamelisaurus and other Core Mamenchisaurus-like Taxa; however, in Klamelisaurus there is a low, oblong, roughly striated area distal and slightly medial to the more proximal, bulging scar described above, approximately level with the most prominent point of the deltopectoral crest. Given the presence of dual, adjacent muscle scars, and the inferred close proximity of the insertions of $\mathrm{m}$. deltoideus scapularis and $\mathrm{m}$. latissimus dorsi (Otero 2018), we suggest that the more proximolateral scar (the ' $\mathrm{m}$. scapulohumeralis anterior' scar of Borsuk-Białynicka [1977]) corresponds to the insertion of $\mathrm{m}$. deltoideus scapularis and the more distomedial scar reflects the insertion of the $\mathrm{m}$. latissimus dorsi. Lateral and anterior to both of these muscle scars, an elongate region of low rugosity extends from the proximolateral corner of the humerus along the lateral side of the deltopectoral crest, fading distally as the crest begins to taper. This region probably corresponds to insertions for $\mathrm{m}$. deltoideus clavicularis (Remes 2008, figs 8-13; Otero 2018), and is abruptly separated from a similar rugosity on the anterolateral edge of the deltopectoral crest by a smooth, elongate groove ( $73 \mathrm{~mm}$ long and $4 \mathrm{~mm}$ wide), termed the 'paramarginal sulcus' by McPhee et al. (2014; Fig. 18G). This sulcus was suggested to be an autapomorphy of Antetonitrus (Yates \& Kitching 2003; McPhee et al. 2014), but is present in some other non-sauropod sauropodomorphs (McPhee et al. 2014) as well as in some sauropods, including Cetiosaurus (OUMNH J.13612), Omeisaurus tianfuensis (ZDM ?T5701), Turiasaurus (CPT 1195) and Bellusaurus (IVPP V17768). Anterior to the paramarginal sulcus, roughly textured bone wraps around the deltopectoral crest anteromedially, up to the border of the anterior fossa (Fig. 18F); the lateral and anterior portions of this rugosity presumably correspond to insertions for $\mathrm{m}$. supracoracoideus while the more medial portions reflect insertions of $\mathrm{m}$. pectoralis (Borsuk-Białynicka 1977; Remes 2008; Otero 2018).

The distal end of the humerus has been partially reconstructed and this region must be interpreted cautiously. In particular, the impression of strongly separated radial and 
ulnar condyles of the distal humerus could not be verified. Just above the reconstructed distal end, the anterior surface of the humerus bears two median ridges, or anterodistal processes, which are present in most sauropods (Upchurch et al. 2015). The anconeal fossa has been crushed inward and is largely obscured by the armature. Distally, the humerus develops a distinct medial surface; no such surface is present laterally, where instead the meeting of the anterior and posterior aspects of the humerus produces a low longitudinal ridge, which is far less distinct and flange-like than the laterodistal margin in $Z b y$ (Mateus et al. 2014). Thus, just above the distal articular surface, the cross section of the humerus is roughly triangular. The flat medial face exhibits fine striations and rugosities.

\section{Ulna}

The right radius and ulna are bound together in a metal armature (Fig. 19). The elements are not in full articulation, but are positioned in approximate anatomical correspondence to each other (see Radius), with a gap between them. The ulna has experienced some weathering at its distal end, but is otherwise essentially intact (Table 8 ). We describe the ulna with the long axis of the posterior process orientated anteroposteriorly.

The ulna is triradiate, with distinct anterolateral and anteromedial processes enclosing a radial fossa positioned opposite the posterior process (Fig. 19A). As in other Core Mamenchisaurus-like Taxa (Chuanjiesaurus - Sekiya 2011, figs 40, 41;

Mamenchisaurus youngi - Ouyang \& Ye 2002, fig. 36), but unlike in Omeisaurus tianfuensis (ZDM ?T5701), Cetiosaurus (OUMNH J.13612), Anhuilong (Ren et al. 2018) and Bellusaurus (IVPP V17768), the angle between the long axes of the anterolateral and anteromedial processes is $<80$. Zhao (1993) described the proximal end of the ulna as autapomorphic in its degree of expansion, but the ratio of the maximum proximal dimension to the proximodistal length of the ulna $(\sim 0.36)$ is comparable to that in various other eusauropods (e.g. Shunosaurus, $\sim 0.36$ [Y. Zhang 1988]; Chuanjiesaurus, 0.36 [Sekiya 2011]; Omeisaurus maoianus, $\sim 0.34$ [Tang et al. 2001]; Vouivria, 0.35 [Mannion et al. 2017]) and less than in Bellusaurus ( 0.43; IVPP V17768) and Opisthocoelicaudia (\$0.47; Borsuk-Białynicka 1977), among other sauropods. Following the measurement protocol of Upchurch et al. (2015), the anteromedial process is only slightly longer than the anterolateral one in Klamelisaurus (Table 8). As in most non-titanosaurian sauropods (Wilson \& Sereno 1998; Mannion et al. 2013; Upchurch et al. 2015), Klamelisaurus lacks an olecranon process projecting above the proximal articular surface. The anteromedial process of the ulna is dorsally convex along its length, rising roughly $10 \mathrm{~mm}$ above the level of the proximal surface of the posterior process before sloping strongly distally below this level. The angle of this distal slope (with respect to the proximal surface of the anteromedial process) is 
less extreme than in Tehuelchesaurus ( $\sim 31$ vs $\sim 40$; Carballido et al. 2011, fig. 16; Fig. 19C). The proximal surface of the anterolateral process is straight to gently concave and projects ventrally towards its tip ( 47); a ventrally sloping anterolateral process is also present in Haestasaurus and some titanosaurians (Upchurch et al. 2015), as well as in Bellusaurus (IVPP V17768). The posterior process separates the fossae on the posterolateral and posteromedial aspects of the ulna. Both of these fossae contain rugose longitudinal striations that merge with similarly textured bone on the posterior process. These fossae dissipate into the surface of the ulna by roughly mid-length. In lateral view, the shaft of the ulna is essentially straight, unlike the sigmoid curvature of Omeisaurus tianfuensis (ZDM ?T5701) and Anhuilong (Ren et al. 2018). Distally, the posterolateral surface of the ulna is expanded (Fig. 19D), and Klamelisaurus has the posteriorly expanded distal end typical of most non-titanosauriforms (D'Emic 2012; Mannion et al. 2013). The posterolateral surface exhibits fine striations, as well as a small, raised bump of unclear significance just below the more distal of two transverse breaks in the shaft of the ulna (Fig. 19D).

Proximally, the radial fossa is weakly rugose. The anterior surface of the ulna becomes smooth and forms a shallow longitudinal channel as the anteromedial and anterolateral processes taper into the shaft of the ulna distally. Towards the distal end of the ulna, the anterior, radial surface becomes flatter and, near the level of the interosseous ridge, twists slightly around the shaft of the ulna to face somewhat more medially. The interosseous ridge (approximately $64 \mathrm{~mm}$ long and $21 \mathrm{~mm}$ wide) is marked by longitudinal striations and is not as longitudinally extensive on the ulna or radius as it is in various macronarians (Curry Rogers 2005; Mannion et al. 2013).

The distal articular surface is roughly pear-or comma-shaped: in distal view, a blunt point - the distal remnant of the anteromedial process - is present anteromedially, the posterior surface is broadly rounded, and the anterior surface is gently concave for reception of the radius (Fig. 19E), as in most non-titanosaurian sauropods (Upchurch et al. 2015; Poropat et al. 2016).

\section{Radius}

The radius is mounted slightly out of its proper articulation with the ulna. This is most apparent in proximal view, where a 30 counter-clockwise rotation of the radius would bring its shallowly concave posterolateral face into tight contact with the slightly convex internal surface of the anterolateral process of the ulna (Fig. 19A), both of which bear low, rugose striations. This rotation also brings the distal interosseous ligament scars of the radius and ulna into closer alignment. We therefore describe the radius as if it were rotated in this way. With the long axis of the posterior process of the ulna orientated anteroposteriorly, the radius is wholly anterior to the ulna, and distally 
rotates slightly to be situated somewhat anteromedial to the ulna. See Table 8 for measurements.

The proximal articular surface is roughly quadrangular, with gently concave posterolateral and posteromedial margins, a slightly convex anterolateral margin and a more strongly convex anteromedial margin (Fig. 19A), and is similar in outline to the proximal articular surface in Ferganasaurus (Alifanov \& Averianov 2003, fig. 8E). The posterolateral and posteromedial margins unite at a blunt, rounded corner that fits into the deepest portion of the radial fossa. Although the shaft expands gently medially towards the proximal articular surface (see below), the radius appears to lack a welldeveloped, medially flaring proximal medial process (Fig. 19C); this process is prominent in Vulcanodon (Cooper 1984, fig. 6), Bellusaurus (IVPP V17768) and many neosauropods (Upchurch et al. 2015, figs 9,10), but is absent in some early branching eusauropods (e.g. Mamenchisaurus youngi - Ouyang \& Ye 2002, pl. XVII;

Omeisaurus tianfuensis - ZDM ?T5701; Ferganasaurus - Alifanov \& Averianov 2003, fig. 8; Turiasaurus - CPT 1196).

In anterior view, the lateral margin of the radius is straight along much of its length, but is expanded somewhat proximally, where the posterior corner is cradled by the radial fossa of the ulna, and distally, where expansion of the radius provides a broader distal articular surface for the ulna (Fig. 19C). The medial margin is essentially straight in the distal half of the radius, but is gently undulating proximally owing to the medial expansion of the proximal articular surface and the development of a low, proximodistally elongate bulge for $\mathrm{m}$. biceps brachii and possibly $\mathrm{m}$. brachialis (Borsuk-Białynicka 1977; Remes 2008; Otero 2018; Fig. 19C). Such strong development of this muscle scar was previously suggested to characterize Titanosauriformes (Upchurch et al. 2015). Subequal to the distal extent of this muscle scar, the anterior surface of the radius becomes flat, such that the midshaft cross section of the radius is essentially ' $\mathrm{D}$ '-shaped. Distally, this flat surface becomes shallowly concave between two low, vertical ridges, and exhibits longitudinal striations (Fig. 19C). A distal anterior fossa is uncommon among sauropods and has been regarded as an autapomorphy of Haestasaurus (Upchurch et al. 2015). Distal to and in line with the $\mathrm{m}$. biceps brachii muscle scar is an additional scar of unclear identity, just above and partly covered by the metal armature. Distally, the posteromedial edge of the radial shaft preserves a pronounced, elongate interosseous ridge $(90 \mathrm{~mm}$ long and $25 \mathrm{~mm}$ wide) that corresponds to a complementary ridge on the opposing face of the ulna (Fig. 19B, C).

The distal end of the radius is incompletely preserved, and it is not possible to ascertain whether the distal surface was bevelled or if it had separate articular condyles, as in some neosauropods (Upchurch et al. 2015). The distal articular surface is not elongate 
in any dimension; with the exception of a concave anterior edge, corresponding to the distal anterior fossa of the shaft, and a somewhat flattened posterolateral surface, for articulation with the ulna, the outline is roughly sub-circular and equidimensional, as in Ferganasaurus (Alifanov \& Averianov 2003, fig. 8F).

\section{Carpals}

The original description of Klamelisaurus mentioned two carpals (Zhao 1993), neither of which could be located by us for study. In the absence of figures, the descriptions of these elements by Zhao (1993) are difficult to confirm (see Supplementary Table 7).

\section{Manus}

Zhao (1993) noted the preservation of seven metapodial bones, including six nonungual elements that he considered to be manual phalanges on the basis of their size and morphology and a single large left ungual he considered to be from the left first digit of either the manus or pes. We likewise identified six metapodial elements and a single ungual from our survey of the preserved material, all of which are bound in metal armatures alongside wholly reconstructed elements of the manus and pes, often obscuring portions of the genuine material. Unlike Zhao (1993), we interpret these as pedal elements and describe them below (see Pes).

\section{Ilium}

The ilium is incomplete (Fig. 20). Most of the iliac blade has been substantially restored, and a clear line separates the roughened texture of bone and matrix from the smooth, reconstructed portions. The distal ends of the pubic and ischial peduncles are missing and the ischial peduncle has been worn anterolaterally, producing an elongate, trough-like depression that interrupts the outer margin of the acetabulum posteriorly. Like the scapulocoracoid, the internal surface of the ilium has been covered with a painted plaster jacket and is affixed to metal armature. Measurements are provided in Table 9.

With the exception of a small stretch of original bone at the level of the ischial peduncle, the entire dorsal surface of the ilium has been reconstructed, prohibiting verification of Zhao's (1993) assertion that the dorsal margin is straight. Where the contour of the original bone can be faithfully interpreted, the supracetabular portion of the ilium is gently concave anteroposteriorly. A distinct, medially instepped shelf is present just dorsal to the acetabulum, although the medial offset of this shelf might have been exaggerated by damage to this region. 
Zhao (1993) described the preacetabular lobe as being bluntly rounded, but this cannot be verified owing to substantial reconstruction at the distal end of this process. The angle of the anterior edge of the pubic peduncle with the ventral margin of the preacetabular lobe is $\$ 90$, while the pubic peduncle is at a slightly acute angle with respect to the long axis of the ilium. The preacetabular lobe projects anterolaterally at an angle of $\$ 20$. A pronounced anterolateral projection of the preacetabular lobe is present in all eusauropods other than Shunosaurus, Cetiosaurus (OUMNH J.1362213623) and Cetiosauriscus (NHMUK PV R3078) (Wilson \& Sereno 1998; Upchurch et al. 2004).

The acetabulum is transversely widest just anterior to its highest point dorsoventrally (when the pubic and ischial peduncles are resting on a horizontal plane), near to the base of the pubic peduncle. The transverse width of the pubic peduncle is greatest proximally and its anteroposterior thickness remains largely unchanged along its preserved length. At the base of the pubic peduncle, the lateral surface of the process is weakly excavated; although the bone in this region has been crushed, this excavation appears to be genuine, and we interpret it as an incipient expression of the subtriangular fossa that is present in various eusauropods, including Cetiosaurus (OUMNH J.13622-13623), Cetiosauriscus (NHMUK PV R3078), Omeisaurus tianfuensis (ZDM T5701), Jobaria (specimen housed at the University of Chicago, labelled 'Tw 77'), Haplocanthosaurus (Tschopp et al. 2015a) and some flagellicaudatans (Tschopp et al. 2015a). Damage to the ischial peduncle obscures the precise outline of its distal end, but its long axis seems to be directed somewhat posterolaterally. Even allowing for the incompleteness of the distal end of the pubic peduncle, a chord projected through the distal articular surfaces of the pubic and ischial peduncles would almost certainly have passed below the ventral margin of the postacetabular lobe, as in most non-somphospondylans (Upchurch 1998; Poropat et al. 2016).

Pubis

The original description of Klamelisaurus noted the presence of an incomplete right pubis, consisting of a flattened shaft with a smooth, concave medial surface and roughened lateral surface (Zhao 1993). Unfortunately, we could not identify any original bone in the reconstructed right pubis included in the mount of Klamelisaurus.

Ischium

Zhao (1993) described briefly a right ischium missing its proximal end, with a broad, flattened midshaft and flat distal end with a gently concave medial surface and rough and convex lateral surface. Little of the original surface of the right ischium is 
apparent, as most of it has been reconstructed with plaster and paint, but a few small regions of whitish-purple bone are exposed on the dorsal surface of the blade proximally. The distal end of the ischium has been weathered and reconstructed, but enough is preserved to suggest that it was little expanded dorsoventrally over the breadth of the shaft, and that the distal surface was sub-triangular, rather than bladelike, with a flat medial surface for the left ischium, a flat dorsomedial surface, and a convex lateral surface.

\section{Femur}

Although Zhao (1993) described only a right femur, which is complete except for a poorly preserved head and partially reconstructed distal condyles, the distal half of a left femur is also stored with the specimen. This second femur clearly belongs to the same animal, given its size and resemblance to the right femur (Figs 21, 22). Metal armature partially obscures the posterior surfaces of both elements. Measurements are provided in Table 10.

The head of the right femur has been weathered and crushed anteroposteriorly. The contour of the lateral margin of the right femur, above the medial deflection of the shaft, suggests that the original extent of the proximal end was not much greater than preserved currently (Fig. 21). Incomplete preservation does not allow determination of whether the femoral head had a stepped ventral margin. The femur appears to lack a flange-like longitudinal trochanteric shelf on the proximal half of the posterior surface, as in many somphospondylan femora (Otero 2010; Mannion et al. 2013), but the armature and incomplete preservation partially obscure this region. At the level of the lateral bulge, the posterolateral surface of the femur bears longitudinal striations.

The fourth trochanter is a low, elongate, rugose bulge, the most prominent point of which is located within the proximal half of the midshaft, at a position approximately $42 \%$ of the length of the femur (based on estimated femur length; Fig. 21C-E; Table 10). It is situated just medial to the centre of the femur, as in Spinophorosaurus (Remes et al. 2009, fig. 4), Patagosaurus (Bonaparte 1986a, fig. 62), Shunosaurus (Y. Zhang 1988, fig. 53, pl. XIII), Mamenchisaurus youngi (Ouyang \& Ye 2002, fig. 40, pl. XIX) and Euhelopus (PMU 234; Wiman 1929, pl. IV), but unlike the holotype of Chuanjiesaurus (Sekiya 2011, fig. 52), Omeisaurus spp. (Omeisaurus tianfuensis ZDM T5701; Omeisaurus maoianus - Tang et al. 2001; Omeisaurus junghsiensis Young 1939), Mamenchisaurus constructus (Young 1958, fig. 8), Yuanmousaurus (Lü et al. 2006), Eomamenchisaurus (Lü et al. 2008) and other sauropods, in which the fourth trochanter is positioned near or at the medial edge of the shaft. Interestingly, Bellusaurus shows polymorphic development of this character (IVPP V17768). Just 
dorsomedial to the fourth trochanter, a large nutrient foramen pierces the shaft; the foramen is $8 \mathrm{~mm}$ wide and is associated with a dorsally directed channel (Fig. 21C).

Except for the medial surface of the tibial condyle, the distal condyles of the right femur are heavily reconstructed, but both condyles are essentially intact and unrestored in the left femur. A deep intercondylar groove separates the condyles (Fig. 22A). The anterior surface of the distal femur bears a slight fossa just proximal to the condyles and the articular surfaces of the femur curve upwards on to the anterior surface of the shaft. The anterior margins of both condyles gently flare anteriorly as they approach the distal articular surfaces. In the transverse plane, the distal articular surfaces are perpendicular to the shaft long axis (Fig. 22A), as in Bellusaurus (IVPP V17768), the referred specimen of Chuanjiesaurus (Sekiya 2011, fig. 53) and Omeisaurus maoianus (Tang et al. 2001, fig. 39), but unlike the proximolaterally bevelled distal ends of Mamenchisaurus youngi (Ouyang \& Ye 2002, fig. 40, pl. XIX), Euhelopus (PMU 234), the holotype of Chuanjiesaurus (Sekiya 2011, fig. 52), Omeisaurus tianfuensis (He et al. 1988, fig. 52) and other early branching eusauropods (e.g. Shunosaurus - Y. Zhang 1988, fig. 53, pl. XIII; Cetiosaurus - OUMNH J.13615). As in other sauropods, the posterior surface of the fibular condyle exhibits a wide, shallow longitudinal groove, bounded medially and laterally by low ridges (Upchurch et al. 2004); the medial ridge is more strongly expressed than the lateral ridge (Fig. 22C, D). The tibial condyle extends farther posteriorly than the fibular condyle (Fig. 22D), the former being at least $20 \%$ longer than the latter (a ratio of 1.24), unlike Mamenchisaurus youngi (Ouyang \& Ye 2002, fig. 40) or Bellusaurus (IVPP V17768) but as in Euhelopus (Wiman 1929), Dongbeititan, Daxiatitan, Tastavinsaurus, an isolated femur from the Tendaguru Formation (Mannion et al. 2019) and saltasaurid titanosaurians (Upchurch et al. 2004; Mannion et al. 2013). The tibial condyle is also substantially narrower mediolaterally than is the fibular condyle (a ratio of 0.79), as in Euhelopus (PMU 234), Omeisaurus tianfuensis (ZDM T5701) and many titanosauriforms (Wilson 2002; Poropat et al. 2016). The medial surface of the distal femur, across the tibial condyle, is irregular and highly rugose.

\section{Tibia}

The right tibia, fibula and astragalus are bound together in a metal armature. These elements are so heavily covered with plaster and paint as to be almost entirely uninformative morphologically, although enough original bone can be discerned along the length of the tibia to allow estimation of several linear measurements, including its length (Table 10). However, the proximal part of a left tibia - not mentioned by Zhao (1993) - is included with the rest of the specimen. It is bound in a metal armature with part of a left fibula - also not mentioned by Zhao (1993) - and a wholly reconstructed astragalus. We base the description of the tibia and fibula on these elements (Fig. 23). 
The proximal articular surface of the tibia is broader transversely than it is anteroposteriorly, and the cnemial crest projects primarily laterally, as in most other eusauropods (Wilson \& Sereno 1998; Upchurch et al. 2004). A fossa embays the proximal articular surface longitudinally. In proximal view, the tibia has a broadly rounded anteromedial margin, while the anterolateral and posterolateral edges are nearly flat and meet at $\sim 90^{\circ}$. The anteromedial face of the proximal end of the left tibia is slightly weathered. The cnemial crest is a stout flange, the distal end of which has suffered some weathering. It does not extend far laterally, but obscures the weak anterior crest of the fibula in anterior view. The proximal lateral edge of the tibia forms a weak lip that overhangs the internal fossa for the fibula; no pinched projection - the 'second cnemial process' (Bonaparte et al. 2000) - is present along this edge. The second cnemial process is also absent in Bellusaurus (IVPP V17768), Euhelopus, Jobaria, Janenschia and various neosauropods (Mannion et al. 2013, 2017, 2019).

\section{Fibula}

The proximal part of a left fibula is preserved in articulation with the tibia (Fig. 23). Its anterior surface has been slightly crushed inward and the posterolateral portion of the proximal end has been weathered. In proximal view, the fibula is roughly semicircular, with a convex anterolateral margin and a relatively flat posteromedial margin. The proximal articular surface is depressed by a shallow fossa. The articulation of the fibula with the tibia appears to be anatomically accurate: the anterior half of the posteromedial margin of the fibula is closely applied to the complementary lateral surface of the tibia, and the weakly developed and anteriorly worn anteromedial crest of the fibula projects behind the cnemial crest of the tibia, as in other sauropods. The long axis of the proximal end of the fibula is approximately perpendicular to that of the tibia. See Table 10 for measurements.

\section{Astragalus}

Although the right astragalus is largely obscured (see above), it is clear that it caps most or all of the distal end of the tibia medially, as in most non-titanosauriforms (Wilson \& Upchurch 2009; Ksepka \& Norell 2010; Mannion et al. 2013). Measurements are provided in Supplementary Table 8.

\section{Calcaneum}

Zhao (1993) noted the presence of a small, irregularly shaped and incompletely preserved right calcaneum, in articulation with the astragalus, but we could not identify this element in the mount. 
Pes

The seven metapodial elements described by Zhao (1993) belong to the pes. Among these elements, we identify a right metatarsal I, a metatarsal II or III, four pedal phalanges and a right pedal ungual, probably from the first or second digit. For convenience, we describe these elements in a plantigrade position, with their proximodistal axis orientated in a horizontal plane (Fig. 24). Measurements for all of these elements are provided in Table 11.

Right(?) metatarsal I. This element is included in the mount as right metatarsal I, a designation with which we agree tentatively. Its dorsal, medial and proximal surfaces are well exposed and mostly unreconstructed, but adjacent reconstructed elements and the armature obscure its distal, lateral and ventral surfaces. Metatarsal I is short and stout. Its proximal articular surface is dorsoventrally tall and sub-quadrangular, although a rounded dorsomedial corner gives the proximal surface a faintly ' $\mathrm{D}$ '-shaped outline, typical of the metatarsal I in other sauropods (Upchurch et al. 2004; Fig. 24A). This surface appears to be mostly intact, with the rough and cratered texture typical of sauropod long bone articular surfaces, including several distinct pits at the dorsomedial corner. The proximal articular surface slopes anterodorsally, as is apparent in medial view (Fig. 24B), but the transverse dimension of the proximal surface is essentially perpendicular to the long axis of the shaft, lacking the distomedial canting of the proximal articular surface present in most eusauropods (Wilson 2002), including Bellusaurus (IVPP V17768), Cetiosauriscus (NHMUK PV R3078) and Euhelopus (PMU 234). The dorsolateral corner of the proximal articular surface bears a shallow concave facet that is overhung dorsally by a small, prong-like projection, presumably to accommodate metatarsal II. The dorsal surface of the shaft lacks the conspicuous nutrient foramina present in Cetiosauriscus (NHMUK PV R3078), Bellusaurus (IVPP V17768) and some flagellicaudatans (Tschopp et al. 2015a), and also lacks the dorsolateral rugosity present in most flagellicaudatans (Upchurch 1995; Bonnan 2005; Tschopp et al. 2015a). Towards the distal articular surface, the dorsal surface of metatarsal I develops two shallow fossae (Fig. 24C). Owing to reconstruction and articulation with a wholly fabricated phalanx, it is not possible to tell whether the distal articular surface is bevelled proximomedially, as in most sauropods (Wilson 2002), and the strongly concave distal margin depicted in dorsal view (Fig. 24C) is an artifact of digitally cropping away the fabricated phalanx. It is also not possible to tell whether Klamelisaurus has the tab-like ventrolateral process of the distal end present in Omeisaurus tianfuensis (He et al. 1988, fig. 55B, D, pl. XVII), Omeisaurus maoianus (Tang et al. 2001, fig. 42), Cetiosauriscus (NHMUK PV R3078; Tschopp et al. 2015a, fig. 105), Bellusaurus (IVPP V17768), flagellicaudatans (Upchurch 1995; Tschopp et al. 2015a) and some titanosauriforms (D’Emic et al. 2011; Mannion et al. 2013). 
Distally, both the medial and lateral edges of the dorsal surface expand dramatically, such that the distal end is notably broader than the proximal end. On its medial side, this expansion is limited to the dorsomedial edge, which develops into a flange-like ridge that overhangs the medial surface of the shaft. We interpret this ridge to be an autapomorphy of Klamelisaurus.

Metatarsal II or III. This element is included in the mount as right metacarpal IV, but we identify it as metatarsal II or III, possibly from the right side. The entire proximal articular surface appears to be composed of plaster and paint, although it is possible that the reconstructed portion captures the general outline of the proximal shaft (Fig. $24 \mathrm{E})$. As reconstructed, the element is transversely rectangular in proximal view, which is unusual for a sauropod metatarsal. If this outline is accurate, it most closely resembles the metatarsal II of Cetiosauriscus (NHMUK PV R3078) and Shunosaurus (Y. Zhang 1988, fig. 55). The strongly concave lateral and medial margins of the shaft are reminiscent of metatarsals II and III of Euhelopus (PMU 234), Camarasaurus (Tschopp et al. 2015b, fig. 23) and Apatosaurus (Gilmore 1936, figs 25, 27), metatarsal II of Shunosaurus (Y. Zhang 1988, pl. XIV-2) and metatarsals II-IV of Jobaria (cast of MNBH TIG 3 at the University of Chicago). The lateral and medial surfaces of the shaft are relatively smooth, but the dorsal surface is marked by a narrow band of rugosity along its midline (Fig. 24D); a similar feature is present in metatarsals I-III of Turiasaurus (CPT 1246-1248) and most flagellicaudatans (Upchurch 1995; Bonnan 2005; Tschopp et al. 2015a), and a shallow striated depression is present on the distal dorsal surface of metatarsal III of Antetonitrus (McPhee et al. 2014). Immediately proximal to this rugose line is a smooth, shallow fossa, while distally the dorsal surface of the element is generally rugose. The shape of the distal articular surface is obscured by a reconstructed phalanx.

Left(?) pedal phalanx III-1(?). The phalanx is squat, blockish and distinctly broader than proximodistally long (Fig. 24F, G). It is largely complete, although a portion of its proximodorsal margin has been chipped away and the ventral and distal surfaces are obscured by plaster. It is therefore unclear whether the distal articular surface was divided into low condyles. Both the proximal and distal articular surfaces preserve a flared lip that circumnavigates the portion of their visible margins. The presumed medial surface is taller than the lateral surface, suggesting that this is a left element. Its shaft is gently waisted and the medial margin is markedly concave. A distinct, shallow, longitudinal fossa is present on the dorsomedial edge of the element (Fig. 24F, G).

The squat form, waisted midsection and lipped margins are most reminiscent of pedal phalanges II-1 and III-1 of Turiasaurus (CPT 1252-1253), III-1 of Mierasaurus (RoyoTorres et al. 2017, fig. 51) and, to a lesser degree, II-1 and IV-1 of Camarasaurus (Tschopp et al. 2015b, figs 26,29). They are also similar in dimensions and shape to 
manual phalanges III-1 and IV-1 of Camarasaurus (USNM 13786; Tschopp et al. 2015 b, figs 18, 19), although in these elements, the distal articular surface is notably wider than the proximal articular surface, a condition not seen in Klamelisaurus. We tentatively identify this element as left pedal phalanx III-1, in accord with the position in which it is mounted.

Other non-ungual ?pedal phalanges. Three other elements, included in the mount as right pedal phalanx III-1, left pedal phalanx IV-3 and right manual phalanx IV-2, appear to include at least some original bone, but their morphology is obscured by poor preservation, plaster and adjacent elements. We note that these elements are all broader transversely than long proximodistally and provide approximate measurements for pedal phalanges IV-3 and III-1 in Table 11.

Right pedal ungual. A large, recurved ungual is preserved, included in the mount as right pedal ungual II-3, but it could also be from digit I or III. It is largely complete, missing only a small portion of its distal tip. It is obscured ventrally by the armature and its proximal end is covered by plaster and a reconstructed penultimate phalanx, so it is not possible to determine the extent to which the proximal articular surface is bevelled. The length of the ungual is greater than the length of metatarsal I.

As in other sauropods, the medial surface of the ungual is convex anteroposteriorly and dorsoventrally, while its lateral surface is flat to weakly concave in these orientations. In dorsal view, the ungual tapers distally and curves very slightly laterally (Fig. 24J). Distinct attachment grooves are present on the medial and lateral surfaces (Fig. 24H, I). On the lateral surface, the attachment groove generally follows the curvature of the ungual; distally, it undercuts the dorsal surface of the ungual before intersecting this surface, tapering out before reaching its distal tip. The medial groove is positioned much farther ventrally than the lateral groove and is less deep overall. Like its lateral counterpart, the medial groove does not extend to the distal tip of the ungual, but tapers into the ventral edge of the ungual distally instead.

Phylogenetic methods and materials

\section{Data matrices}

We assessed the relationships of Klamelisaurus gobiensis using the character matrices of Carballido et al. (2015) (hereafter 'CEA') and Gonzalez Riga et al. (2018) (hereafter 'GEA'), both of which include a broad sample of eusauropod diversity. In addition to Klamelisaurus, we added several other taxa to these matrices (Supplementary Table 1). We scored Antetonitrus, Massospondylus and Pulanesaura in the CEA matrix and Cetiosaurus oxoniensis in the GEA matrix. To both matrices, we added the Haute 
Moulouya taxon, Cetiosauriscus and numerous East Asian sauropods and putative 'mamenchisaurids', some of which (marked with an asterisk) have not previously been included in a published phylogenetic analysis, including Mamenchisaurus constructus, Mamenchisaurus sinocanadorum, the holotype and a referred specimen of Mamenchisaurus hochuanensis, Wamweracaudia keranjei, the holotype and referred specimens of Chuanjiesaurus anaensis, Qijianglong guokr, Tienshanosaurus chitaiensis, the Phu Kradung taxon, a series of cervicodorsal vertebrae from the Shishugou Formation (hereafter 'Shishugou cervicodorsal vertebrae'), the holotype of Omeisaurus junghsiensis, Omeisaurus tianfuensis and Omeisaurus maoianus. Our sampling of putative 'mamenchisaurids' emphasized inclusion of single-specimen operational taxonomic units (OTUs) rather than genus-level amalgamations of presumed conspecific taxa. This approach allows for finer tracing of character evolution within 'mamenchisaurids' and is particularly warranted in light of recent phylogenetic analyses that have failed to recover monophyletic groupings of both Mamenchisaurus spp. and Omeisaurus spp. (Sekiya 2011; Xing et al. 2015; Ren et al. 2018). Unlike previous authors (Sekyia 2011; Xing et al. 2015; Ren et al. 2018), we include the holotype and referred specimens of Chuanjiesaurus as separate OTUs in our phylogenetic analyses because we have observed several anatomical differences between them (see Supplementary material).

In addition to the taxa listed above, we revised character scorings for several taxa included in the GEA and CEA matrices based on personal observations and photographs; these changes are detailed in the Supplementary material. Given the controversial taxonomic history of Euhelopus, we detail and justify numerous character state changes for this taxon (see 'Revised scorings for Euhelopus' in the Supplementary material). Sources of comparative information for newly scored taxa and characters are detailed in Supplementary Tables 1 and 9. Character matrices were assembled in Mesquite v. 3.2 (Maddison \& Maddison 2018); input files for phylogenetic analyses, which include all character scorings for the CEA and GEA matrices, are provided in the Supplementary material, Appendices 1-6. The final CEA matrix comprised 94 taxa and 385 characters, and the final GEA matrix comprised 103 taxa and 436 characters (Supplementary material, Appendices 7,8).

As in previous phylogenetic analyses of the data sets included here, trees were rooted on Plateosaurus and Shunosaurus for the CEA and GEA matrices, respectively. Multistate character ordering followed previous analyses of these matrices (except as detailed in Supplementary Table 10), and included only those characters with nested states or a clear transformation series. Following the analysis of Gonzalez Riga et al. (2018) and previous iterations of that matrix (Mannion et al. 2013, 2017; Upchurch et al. 2015; Poropat et al. 2016), nine highly unstable and incomplete taxa 
(Astrophocaudia, Australodocus, Brontomerus, Fukuititan, Fusuisaurus, Liubangosaurus, Malarguesaurus, Mongolosaurus, Tendaguria) were excluded a priori from analyses of the GEA matrix. After conducting analyses, character optimization was performed in TNT v. 1.5 and Mesquite v. 3.2.

New and amended characters

We reformulated seven characters in the CEA matrix and six characters in the GEA matrix, as detailed in Supplementary Table 10. Revised versions of these characters were added to the end of the matrix. To preserve character numbering across iterations of the CEA and GEA matrices, the previously formulated versions of these characters were not removed from the matrix but were made inactive prior to phylogenetic analysis. In addition, we added numerous characters to the CEA and GEA matrices, 10 of which are new and have not previously been included in a phylogenetic analysis. These are detailed in the Supplementary material.

\section{Revised scorings for Euhelopus}

Given the controversial taxonomic history of Euhelopus, we detail and justify numerous character state changes for this taxon, including our interpretation that Euhelopus lacks a preantorbital opening (see 'Revised scorings for Euhelopus' in the Supplementary material). All changes to character scorings are provided in the Supplementary material, along with those for other taxa.

\section{Analytical approaches}

To test the phylogenetic affinities of Klamelisaurus and other Middle-Late Jurassic East Asian eusauropods, we conducted both equal- and implied-weights parsimony analysis, as well as tip-dated Bayesian inference. We discuss our use of multiple optimality criteria in the Supplementary material.

Parsimony analyses. Phylogenetic analyses under a parsimony optimality criterion were performed using TNT v. 1.5, with both equal and extended implied weighting of characters (Goloboff et al. 2003, 2008; Goloboff 2014; Goloboff \& Catalano 2016). For extended implied weighting, we used a concavity constant $(\mathrm{K})$ of 12 ; higher values of $\mathrm{K}$ less severely down-weight homoplasious characters than does the default value of 3 in TNT, and $\mathrm{K}=12$ was recently found to outperform lower $\mathrm{K}$ values in simulation analyses (Goloboff et al. 2017). Heuristic searches were conducted using 'New Technology' algorithms: 50 search replications were used as a starting point for each hit, and were run until the best score was hit 10 times, using random and constraint sectorial searches under default settings, five ratchet iterations and five rounds of tree fusing per replicate ('xmult=replications 50 hits 10 css rss ratchet 5 fuse 5'). The most 
parsimonious trees (MPTs) resulting from the primary search were subjected to an additional round of tree bisection and reconnection (TBR) branch swapping to ensure a thorough sampling of MPTs, with the maximum number of trees in memory set to 200,000 . While there may be more than 200,000 MPTs, our experience suggests that including more trees will not change the consensus calculation. To identify unstable OTUs, we implemented the iterative reduced positional congruence protocol (PCR) protocol of Pol and Escapa (2009), using the iterpcr.run script in TNT v. 1.5.

To assess branch support, we conducted symmetric resampling, which is appropriate for analyses in which characters have different prior weights (as with implied weighting or matrices with additive characters; Goloboff et al. 2003). For both equaland implied-weights parsimony analyses, we report both the absolute and the "Groups present/Contradicted" (GC) frequencies of clades (i.e. the difference in frequency between the recovered group and its most frequent contradictory resampled group; Goloboff et al. 2003) on reduced strict consensus trees, based on 1000 replications. For equal-weights analyses, we also calculated decay indices as a measure of node support, collecting 100,000 suboptimal trees for each additional step in tree length.

Bayesian analyses. Time-calibrated Bayesian analyses were conducted in MrBayes v. 3.2.6 (Nylander et al. 2004), with terminal taxa treated as non-contemporaneous tips. For the root age of the CEA and GEA trees, we used uniform priors on the age of Sauropodomorpha (Ladinian-Carnian) and Eusauropoda (Norian-Hettangian), respectively, following recent discussion and fossil discoveries informing the age of these groups (Langer 2014; Nicholl et al. 2018). For the OTUs, ages were enforced as a uniform distribution on the age of the tip; these distributions are based on a survey of the geological and palaeontological literature. A wealth of studies in the last several decades has provided refined chronostratigraphic constraints on the age of many sauropod specimens; where possible, we constrained the ages of specimens using data from studies that conducted radiometric dating, rather than relying solely on the stated age of the specimen in the descriptive literature. Chronostratigraphic data and relevant sources are provided in Supplementary Table 9.

To model morphological evolution, we considered two different models: the Mkv model of Lewis (2001) (i.e. the Mk model, conditioned on the inclusion of only variable characters in the matrix), and a recently developed extension of that model, the MkA model (Pyron 2016). Under the Mkv model, the frequencies and rates of all character states are equivalent. By contrast, the MkA model treats the codification of binary characters as biologically meaningful for those characters for which a confident a priori hypothesis of plesiomorphy and apomorphy can be made: if binary characters are coded with meaningful state labels, wherein 0 is plesiomorphic and 1 is apomorphic, then state frequencies and thus asymmetric rates of forward and backward 
transitions between plesiomorphy and apomorphy can be estimated. The MkA model thus incorporates the biological reality that state frequencies are not evenly distributed in morphological matrices, and has produced higher marginal model likelihoods for morphological matrices that include fossils than have analyses using only the Mkv model (Pyron 2016).

The subset of binary characters for which a strong prior hypothesis of plesiomorphyapomorphy could be made were included in their own MkA partition, while all other binary characters and all multistate characters were included in an unlinked Mkv partition, following Pyron (2016). Each partition received its own gamma-distributed rate heterogeneity parameter, to accommodate among-character rate variation. To test the relative fit of the MkA + Mkv over the Mkv-only approach, we

used Bayesian stepping-stone analysis; this method provides a more accurate estimation of the marginal likelihood of a model than does the harmonic mean calculated from the posterior distribution (Xie et al. 2011). We used the resulting marginal likelihoods to compute Bayes factors (calculated as twice the difference of the marginal log likelihoods of competing models) for model comparison. Because stepping-stone analyses are computationally intensive, we executed these analyses on matrices in which the age of the fossil was fixed at the youngest age of its uniform distribution (Supplementary Table 9), implementing four runs of four chains each for $200,000,000$ generations over a total of 50 stepping-stone samples. For the CEA matrix, the marginal model likelihood was 5687.65 for the analysis including the MkA model and 5726.88 for the Mkv-only analysis, for a Bayes factor of 78.46; for the GEA matrix, these likelihoods were 7155.64 and 7208.24, respectively, for a Bayes factor of 105.20. Given that a Bayes factor of $>10$ is usually considered 'very strong' support (Kass \& Raftery 1995), we conclude that, for both matrices, the inclusion of the MkA model produces a much better fit to the data. Thus, for the full time-calibrated Bayesian analyses, we employed the MkA + Mkv models, as described above.

Following C. Zhang et al. (2016), we used the fossilized birth-death prior on branch lengths. The fossilized birth-death prior is a sampled-ancestor process (Stadler 2010; Gavryushkina et al. 2014; C. Zhang et al. 2016), and allows fossils to be direct ancestors of descendant branches. Because no extant taxa are included in either matrix, we added an extant dummy outgroup taxon to each matrix, constrained to one node more basal than Plateosaurus (CEA matrix) and Shunosaurus (GEA matrix). This dummy taxon serves only to provide an extant benchmark for establishing a time scale for the analysis, and represents a hypothetical extant archosaurian relative; it receives no character scorings (i.e. all characters are scored as '?'), and thus does not bear on character polarization. Given the presence of a single (hypothetical) extant 
archosaurian relative, we included a sampling probability of 0.0001 for extant taxa, and specified that sampling was random, rather than diversified.

We followed previous authors (Pyron 2016; C. Zhang et al. 2016) in placing broad, uninformative priors on speciation (exp[10]), extinction (beta[1,1]) and fossilization (beta[1,1]). Because fossil sampling in our analyses ceases at the CretaceousPaleogene boundary, we used piecewise-constant rates in the fossilized birth-death prior, with a rate shift at $66 \mathrm{Ma}$. Rates of morphological evolution were modeled using a relaxed clock approach. For the relaxed clock rates, we used the independent gamma rates (IGR) model (Ronquist et al. 2012) with a broad prior of $\exp (10)$ on the parameter describing rate variance through time, and calculated a broad prior on the base rate of the clock, following Pyron (2016). Each analysis consisted of four runs of the Metropolis-coupled Markov chain Monte Carlo algorithm across four parallelized chains (one cold chain and three heated chains; Altekar et al. 2004) on the George Washington University's Colonial One high-performance computing cluster for at least $100,000,000$ generations, sampling every 5000 generations. Stationarity and appropriate burn-in were assessed in Tracer v. 1.7 (Rambaut et al. 2018). We assumed convergence had been reached when effective sample sizes for all parameters were $>200$.

We present the fully resolved maximum clade credibility trees (MCCT) from the CEA and GEA analyses, produced using TreeAnnotator v. 1.8.4 (Bouckaert et al. 2014). To describe branch support, we report both the posterior probability of branches in the MCCT as well as a recently developed metric termed the transfer bootstrap expectation (or TBE; Lemoine et al. 2018), which we here apply for the first time to summarizing Bayesian posterior distributions of trees and call the 'transfer posterior expectation' (TPE). Although described solely as a modification to traditional bootstrapping approaches, the method employed by Lemoine et al. (2018) is appropriate for any distribution of trees for which a corresponding reference tree (e.g. a maximum likelihood tree or MCCT) is available. Unlike traditional bootstrapping methods, which strictly interpret a given bipartition as either present or absent, the TBE/TPE approach recognizes that an inferred branch is not simply correct or incorrect, and may include errors that reflect the relative lability of constituent tips in the tree distribution (Lemoine et al. 2018). The transfer distance between a given branch of the reference tree and a branch from a tree within the bootstrapped or posterior distribution is equal to the number of taxa that must be moved to make the two clades identical. Using the transfer distance, a separate TBE/TPE score ranging from 0 to 1 is calculated for each node in the reference tree, scaled to the size of the smaller clade in the bipartition, such that a score of 0 indicates that the distribution of trees is random with respect to that node in the reference tree and higher values reflect increasing stability of the members within a given clade, with a value of 1 indicating 
that the bipartition is present in all trees in the distribution (Lemoine et al. 2018). The method is thus less sensitive to variability in topology induced by rogue taxa than are bootstrap frequencies and posterior probabilities, making it especially suitable to analyses where high lability of some taxa obscures underlying

consistency in topology. For nodes subtending two terminal taxa, the TPE score is equivalent to the posterior probability of the node. TPE values were calculated from a pool of 10,000 trees from the posterior distribution using the BOOSTER web portal (https://booster. pasteur.fr).

\section{Phylogenetic results}

\section{Overview}

Before describing the results of each analysis separately, we first summarize several noteworthy features across analyses (Figs 25-30; Supplementary Table 11). In all of the analyses presented here, Klamelisaurus is a nested member of a core group of predominantly Middle-Late Jurassic Chinese eusauropods that includes Qijianglong, Mamenchisaurus spp. (with the exception of the labile Mamenchisaurus constructus), the holotype and referred specimens of Chuanjiesaurus, Wamweracaudia, the Shishugou cervicodorsal vertebrae and the Phu Kradung taxon, which we refer to as 'Core Mamenchisaurus-like Taxa' throughout. Symmetric resampling support and posterior probabilities for the clade containing these taxa are low, but TPE values from Bayesian analysis of both the CEA and GEA matrices are 90, suggesting that a few relatively unstable taxa mask underlying support for the group. Bayesian analyses also provide moderate support for placement of Tienshanosaurus, Cetiosauriscus and Omeisaurus species as close outgroups of the Core Mamenchisaurus-like Taxa.

The clade containing Klamelisaurus also includes Euhelopus (and is thus termed Euhelopodidae) in all but the extended implied-weights analysis of the GEA matrix, in which Euhelopus is instead recovered as a somphospondylan and Klamelisaurus belongs to a non-neosauropod Mamenchisauridae. Euhelopodidae/ Mamenchisauridae lies outside of Neosauropoda except in the extended implied-weights analysis of the CEA matrix, in which a Euhelopodidae that includes Bellusaurus is found to be an early branching macronarian lineage.

In accord with recent appraisals of Middle-Late Jurassic Chinese sauropod phylogeny (Sekiya 2011; Xing et al. 2015; Ren et al. 2018; see below), none of our analyses recovered a monophyletic Mamenchisaurus or Omeisaurus, suggesting that these genera have become wastebasket taxa in need of systematic revision. In half of the analyses, Mamenchisaurus constructus is recovered within Euhelopodidae (Bayesian 
analysis of the CEA and GEA matrices) or Mamenchisauridae (extended impliedweights parsimony analysis of GEA matrix), whereas it is found outside of these clades in a subset of trees in the remaining analyses. The holotype and referred specimens of Mamenchisaurus hochuanensis are not supported as sister taxa in any of our analyses, a result also recovered by Sekiya (2011). Whereas Sekiya (2011) included the holotype and referred specimens of Chuanjiesaurus as a single OTU, we split them (see Supplementary material). Character score differences between these specimens often result in non-monophyly of the taxon, although a monophyletic Chuanjiesaurus is found in the extended implied-weights analysis of the GEA matrix. Bellusaurus is found to be a macronarian or a diplodocoid in all analyses except the Bayesian analysis of the GEA matrix, in which it is sister to Neosauropoda. Whereas previous studies have recovered Turiasauria just outside Neosauropoda (e.g. Royo-Torres et al. 2006, 2017; Mannion et al. 2019), two of our analyses find the clade within Neosauropoda, as sister to Diplodocimorpha (equal-weights parsimony analysis of the GEA matrix; implied-weights parsimony analysis of the CEA matrix).

The lack of topological consistency between analyses and generally low branch support within Euhelopodidae/ Mamenchisauridae highlights the need for redescriptions of most Middle-Late Jurassic Chinese sauropods, continued development of characters that discriminate taxa within the group, and systematic and taxonomic revision of Mamenchisaurus and Omeisaurus. This lack of topological consistency is troubling, but we suspect that similar levels of uncertainty typify morphological matrices of other fossil lineages.

\section{GEA matrix}

Equal-weights parsimony (GEA-EW). The equal-weights parsimony analysis produced 200,000 MPTs with lengths of 2017 steps (consistency index $[\mathrm{CI}]=0.232$; retention index $[\mathrm{RI}]=0.567$ ). The resultant strict consensus is poorly resolved (Fig. 25A). However, a posteriori pruning of two labile taxa (Mamenchisaurus constructus and Xianshanosaurus) dramatically improves tree resolution (Fig. 25B). The position of $M$. constructus is highly labile within the clade of mostly Chinese eusauropods that includes Klamelisaurus, and across the set of MPTs this taxon occupies nearly every branch above Tienshanosaurus. In a small subset of MPTs, M. constructus is recovered outside of this clade, as sister to Omeisaurus maoianus. Iterative PCR analysis of this and the other parsimony analyses (see below) indicate the labile position of $M$. constructus can be attributed to missing data, as only limited character information can be gleaned from its original description (Young 1954). Xianshanosaurus is recovered in this analysis as either a titanosaurian that is sister to Malawisaurus, or as a turiasaurian more closely related to Mierasaurus and Moabosaurus than to Losillasaurus, Turiasaurus and Zby. 
After pruning Mamenchisaurus constructus, a clade of mostly Chinese eusauropods constituting a non-neosauropod Euhelopodidae becomes well resolved, although nodal support is poor. In a subset of MPTs, Omeisaurus tianfuensis and Cetiosauriscus are recovered in a lineage that is sister to the remaining members of Euhelopodidae, while in other MPTs the O. tianfuensis + Cetiosauriscus lineage is sister to Euhelopodidae + all other eusauropods, or these two taxa are successive outgroups to the latter clade. Near the base of Euhelopodidae is a novel clade of Chinese taxa comprising Dongbeititan, Daxiatitan and Euhelopus. Wamweracaudia and Tienshanosaurus are successive outgroup taxa to a clade comprising two major sister lineages. The first includes Klamelisaurus, the Phu Kradung taxon, the Shishugou cervicodorsal vertebrae, Mamenchisaurus youngi and the referred specimen of M. hochuanensis, while its sister clade includes both the holotype and referred specimens of Chuanjiesaurus, Qijianglong, M. sinocanadorum and the holotype specimen of $M$. hochuanensis. Chuanjiesaurus is not recovered as monophyletic.

Apart from finding Euhelopus, Dongbeititan and Daxiatitan in a non-neosauropod Euhelopodidae, the most notable departures in our results from previous studies are the recovery of Bellusaurus as sister to Diplodocimorpha and the placement of Turiasauria within Diplodocoidea as sister to Bellusaurus + Diplodocimorpha, both of which are novel positions for these taxa.

Extended implied-weights parsimony (GEA-IW). This analysis produced 30 MPTs (tree lengths $=77.66935$ steps; Fig. 26). The most striking difference from the equalweights analysis is that Euhelopus is recovered in a titanosauriform Euhelopodidae as a close relative of Erketu and Gobititan. Bellusaurus is recovered as the earliest branching lineage of Diplodocoidea, and Turiasauria is found to be sister to Neosauropoda. The taxa identified as labile in the equal-weights analysis are more stable under extended implied weighting. Mamenchisaurus constructus is nested in a mostly Chinese, non-neosauropod Mamenchisauridae, although it is highly labile within the clade, and Xianshanosaurus is only recovered within Lithostrotia. Mamenchisaurus constructus can parsimoniously occupy most positions above the node subtending Omeisaurus junghsiensis. Pruning M. constructus resolves the large polytomy in Mamenchisauridae. Interrelationships of the remaining taxa are consistent with results of the equal-weights analysis in favouring close kinship between Klamelisaurus, the Phu Kradung taxon, the Shishugou cervicodorsal vertebrae and $M$. youngi, and between Qijianglong and M. sinocanadorum. The monophyly of $M$. hochuanensis is not supported, and the holotype and referred specimens of the taxon are instead recovered as successive sister taxa of the clade that includes M. youngi, the Shishugou cervicodorsal vertebrae, the Phu Kradung taxon and Klamelisaurus. Chuanjiesaurus is found to be monophyletic, supported by possession of a radius less than 0.65 times the length of the humerus (a feature otherwise restricted to 
titanosauriforms) and medial deflection of the proximolateral margin of the femur. Omeisaurus tianfuensis is found to be more closely related to Turiasauria + Neosauropoda than to mamenchisaurids, while Cetiosauriscus is recovered as an early branching eusauropod.

Time-calibrated Bayesian (GEA-B). The MCCT from the time-calibrated Bayesian analysis recovers Klamelisaurus in a large, non-neosauropod Euhelopodidae that includes Mamenchisaurus constructus (Fig. 27). Posterior probabilities for nodes throughout the clade are low, although TPE values provide relatively strong support for several early branching nodes. The relationships within this clade differ substantially from those found in the parsimony analyses. Cetiosauriscus stewarti and Omeisaurus spp. are recovered within Euhelopodidae in an early branching clade that is sister to all other euhelopodids. Unlike the equal-weights parsimony analysis, a subclade comprising Euhelopus, Daxiatitan, Dongbeititan and (unique to the Bayesian analysis) Xianshanosaurus is in a relatively well-nested position within Euhelopodidae, rather than sister to euhelopodids other than Omeisaurus junghsiensis.

The Bayesian analysis is more similar to the GEA-IW than to the GEA-EW analysis with respect to the branching order of non-neosauropod eusauropods and early branching neosauropods. As in the GEA-IW analysis, Jobaria and Lapparentosaurus are found to be turiasaurians and Turiasauria is outside of Neosauropoda. Atlasaurus, recovered in parsimony analyses as a non-neosauropod eusauropod (GEA-EW) or brachiosaurid (GEA-IW), is found to be an early branching macronarian. Unlike in the parsimony analyses, Bellusaurus is found just outside of Neosauropoda, rather than as sister to Diplodocimorpha.

\section{CEA matrix}

Equal-weights parsimony (CEA-EW). Equal-weights parsimony produced 200,000 trees of length 1349 steps $(\mathrm{CI}=0.341 ; \mathrm{RI}=0.692$; Fig. 28). The strict consensus of the equal-weights parsimony analysis is generally poorly resolved, although a posteriori pruning of several early branching sauropods and East Asian taxa produces a reduced strict consensus with much greater resolution (Fig. 28B). Mamenchisaurus constructus is highly labile and recovered within Euhelopodidae (on any branch above the node subtending Omeisaurus junghsiensis) and, surprisingly, as sister to Isisaurus. Wamweracaudia exhibits the same pattern of parsimonious positions as M.constructus, except that it is never recovered outside of Euhelopodidae. As for M. constructus, iterative PCR attributes the lack of stability in the positions of Wamweracaudia and the Shishugou cervicodorsal vertebrae within Euhelopodidae to missing data. A clade comprising Bellusaurus + Cetiosauriscus is recovered as the earliest branching macronarian lineage. Tienshanosaurus is variably found to be an early branching 
euhelopodid or sister to Bellusaurus + Cetiosauriscus. Jobaria is recovered outside of Euhelopodidae + more derived sauropods, and Haplocanthosaurus is found in a polytomy with Diplodocimorpha and Macronaria. Notably, the Haute Moulouya taxon is recovered outside of Eusauropoda (contra Nicholl et al. 2018).

Pruning the most labile taxa reveals a clade of mostly Chinese eusauropods constituting a non-neosauropod Euhelopodidae. Omeisaurus tianfuensis is found to be the earliest branching euhelopodid; above this node, $O$. junghsiensis is sister to a group comprising the Core Mamenchisaurus-like Taxa, which here also includes O. maoianus and Euhelopus. As in the GEA-EW analysis, symmetric resampling support for Euhelopodidae is very low.

Extended implied-weights parsimony (CEA-IW). Extended implied-weights parsimony produced 24,925 trees (tree length $=50.39402$ steps). In the strict consensus (Fig. 29), Euhelopodidae is entirely unresolved and is in a novel position as an early branching macronarian lineage that includes Bellusaurus, Omeisaurus junghsiensis and Tienshanosaurus. Omeisaurus tianfuensis, which was the earliest branch of a nonmacronarian Euhelopodidae in the equal-weights analysis, is found in a lineage with Cetiosauriscus that is sister to Jobaria + all remaining sauropods. Omeisaurus maoianus and Mamenchisaurus constructus form a novel clade with Cetiosaurus and Patagosaurus. As in the GEA-EW analysis, Turiasauria is recovered within Diplodocoidea, as sister to Diplodocimorpha. As in the GEA-EW and CEA-EW analyses, symmetric resampling support for Euhelopodidae is very low.

Time-calibrated Bayesian (CEA-B). The MCCT from the time-calibrated Bayesian analysis produces a large, non-macronarian Euhelopodidae that is sister to Turiasauria + Neosauropoda (Fig. 30). Unlike the CEA-IW analysis, Euhelopodidae includes all species of Mamenchisaurus and Omeisaurus, as well as Klamelisaurus, Qijianglong, Chuanjiesaurus, Tienshanosaurus, the Phu Kradung taxon, Wamweracaudia and Euhelopus. Posterior probabilities for Euhelopodidae and its basal-most nodes are modest, but TPE values for these branches are all $>90$. Unlike in the CEA-IW analysis, Turiasauria is sister to Neosauropoda, and includes Jobaria and Haplocanthosaurus. As in the parsimony analyses, Bellusaurus is found to be an early branching macronarian, with strong support. The MCCT agrees with the GEA-B analysis in finding Omeisaurus tianfuensis and Cetiosauriscus to be close relatives in an early branching lineage of Euhelopodidae.

Topology tests

To further test the phylogenetic position of Euhelopus, we assessed the relative support for macronarian vs non-neosauropod euhelopodid affinities of Euhelopus using 
constrained topologies. The GEA-IW analysis is the only one in which Euhelopus was not recovered in a Euhelopodidae that includes Klamelisaurus and Core Mamenchisaurus-like Taxa (see above). For the GEA-IW constraint analysis, Euhelopus was forced into monophyly with Klamelisaurus, Mamenchisaurus youngi and other Core Mamenchisaurus-like Taxa; for all other analyses, Euhelopus was constrained to lie within Macronaria, to the exclusion of Core Mamenchisaurus-like Taxa. These constrained topologies were then compared to the best fit topology produced from the unconstrained analyses (Figs 25-30) using Templeton tests (Templeton 1983) for the parsimony analyses and Bayes factor comparisons of marginal model likelihoods produced from stepping-stone analysis (Xie et al. 2011; see above) for the Bayesian analyses. Templeton tests were conducted in TNT using a custom script (Schmidt-Lebuhn 2016).

For the GEA-EW, CEA-EW and CEA-IW analyses, constrained trees are 1, 5 and 0.23108 steps longer, respectively, than the unconstrained trees, and Templeton tests failed to reject topologies in which Euhelopus is a macronarian ( $p>0.05)$. For the GEAIW, the constraint tree is 0.26778 steps longer than the unconstrained tree; as in Wilson \& Upchurch (2009), a Templeton test did not reject the hypothesis that Euhelopus lies outside of Neosauropoda $(\mathrm{p}>0.05)$.

Owing to the computationally intensive nature of stepping-stone analysis, we were unable to achieve convergence across four independent runs of the constrained and unconstrained Bayesian topologies.

\section{Discussion}

Systematics of Klamelisaurus, Euhelopus and Middle-Late Jurassic Chinese sauropods

Klamelisaurus is consistently recovered as a nested member of a clade of mostly Chinese eusauropods. This clade is termed Mamenchisauridae in the GEA-IW analysis; however, in all other analyses, Euhelopus also falls within this lineage, which thus takes the name Euhelopodidae. The inconsistent placement of Euhelopus is symptomatic of the larger disagreement across analyses over the contents and interrelationships of Euhelopodidae/Mamenchisauridae (Figs 25-30). Other notable conflicts include whether Omeisaurus tianfuensis belongs inside or outside this clade, whether Chuanjiesaurus is monophyletic, and even whether the type species of Mamenchisauridae, Mamenchisaurus constructus, is closely related to other named species of Mamenchisaurus. 
This substantial disagreement presents many difficulties for interpretation and comparison of the results, which might be ameliorated if the results of some analyses could be preferred over the others. As discussed in the Supplementary material, the findings of recent simulation studies (Wright \& Hillis 2014; O'Reilly et al. 2016; Goloboff et al. 2017, 2019; Puttick et al. 2017) lead us to favour implied-weights parsimony and Bayesian inference over equal-weights parsimony, as these approaches are expected to yield more accurate topologies. With respect only to relationships within Mamenchisauridae/Euhelopodidae, output from iterative PCR analysis (Pol \& Escapa 2009) of our parsimony results also supports a preference for topologies produced from analyses of the GEA matrix. Although the ratio of characters to taxa is comparable for the two matrices (4.1 for the CEA matrix and 4.2 for the GEA matrix), iterative PCR analysis found the GEA matrix to be better suited than the CEA matrix to resolving the interrelationships of Middle-Late Jurassic East Asian eusauropods. In parsimony analyses of the CEA matrix, but not the GEA matrix, missing data accounted for instability in the positions of Qijianglong, the Phu Kradung taxon, the Shishugou cervicodorsal vertebrae, Wamweracaudia, the referred specimen of Chuanjiesaurus, both specimens of Mamenchisaurus hochuanensis and $M$. sinocanadorum. By contrast, only the unstable position of M. constructus could be attributed to missing data in the GEA matrix.

This reasoning provides a modest basis for favouring the results of the GEA-IW and GEA-B analyses in considering mamenchisaurid/euhelopodid interrelationships. However, even after narrowing down our results to focus on these two analyses, profound topological disagreements remain (Fig. 31). For the focal taxa of this study, consensus between the GEA-IW and GEA-B analyses is limited to recovery of a clade comprising the Core Mamenchisaurus-like Taxa (Klamelisaurus, Wamweracaudia, Qijianglong, Chuanjiesaurus, the Phu Kradung taxon, the Shishugou cervicodorsal vertebrae, most species of Mamenchisaurus) as well as M. constructus, with Tienshanosaurus positioned as a close relative of this core group (Fig. 31).

Comparisons to previous studies focused on mamenchisaurid phylogeny. The most diverse phylogenetic sampling of Middle-Late Jurassic Chinese sauropods to date was provided by Sekiya (2011), Xing et al. (2015) and Ren et al. (2018). Sekiya (2011) added numerous Chinese sauropods and characters to the matrices of Wilson (2002) and Upchurch et al. (2004). Xing et al. (2015) employed a modified version of the matrix of Harris (2006), adding numerous Chinese and putative eusauropod taxa as well as cranial characters derived from Xing et al. (2013). Ren et al. (2018) updated the matrix of Xing et al. (2015) to include Anhuilong and Huangshanlong, although apart from finding these taxa to be more closely related to Omeisaurus tianfuensis than to other mamenchisaurids, the interrelationships of Mamenchisauridae recovered by Ren et al. (2018) are identical to those of Xing et al. (2015). These analyses do not have 
complete taxonomic overlap of Middle-Late Jurassic Chinese sauropods with each other or with the present study, but all of them include Omeisaurus tianfuensis, Chuanjiesaurus, Mamenchisaurus youngi, M. sinocanadorum, the holotype of $M$. hochuanensis and M. constructus (Fig. 31).

None of these previous studies recovered Euhelopus in a non-macronarian Euhelopodidae, and thus referred to the clade of Chinese eusauropods composed of Mamenchisaurus spp. and related taxa as Mamenchisauridae, although these studies did not offer a phylogenetic definition for the group. The GEA-IW and GEA-B topologies (as well as our other phylogenetic results) are consistent with those of Sekiya (2011), Xing et al. (2015) and Ren et al. (2018) in finding Omeisaurus tianfuensis to be outside a core group of Mamenchisaurus-like taxa, either as the sister taxon to all remaining mamenchisaurids (Sekiya 2011: modified matrix of Upchurch et al. 2004; Xing et al. 2015; Ren et al. 2018) or wholly outside this clade (Sekiya 2011: modified matrix of Wilson 2002) (Fig. 31). In addition, the GEA-IW and GEA-B analyses (as well as the CEA-B results) concur with these previous studies in recovering all species of Mamenchisaurus in a clade, to the exclusion of other nonneosauropod eusauropods (e.g. Shunosaurus, Cetiosaurus, Patagosaurus), although the monophyly of Mamenchisaurus is not supported in any analysis.

Beyond these commonalities, there is no consensus across studies on other aspects of mamenchisaurid phylogeny. This is not unexpected, given the differences in character composition and taxonomic sampling across studies, and topological results vary between the two analyses conducted by Sekiya (2011), as well as between analyses of the CEA and GEA matrices conducted here. There are, however, a few additional areas of agreement for taxa that our analyses share with either Sekiya (2011) or Xing et al. (2015) (Fig. 31). First, both the results of Sekiya (2011) and the GEA-IW and GEA-B analyses challenge the referral of ZDM 0126 to Mamenchisaurus hochuanensis by Ye et al. (2001), which was made without reference to a character-based diagnosis of the taxon. Neither set of analyses recovered the monophyly of Mamenchisaurus hochuanensis, and nine additional steps were required to force ZDM 0126 to be the sister taxon of the holotype of Mamenchisaurus hochuanensis in the Upchurch et al. (2004) matrix (Sekiya 2011). Second, the results of the GEA-IW and GEA-B analyses are in accord with those of Xing et al. (2015) and Ren et al. (2018) in failing to support the monophyly of Omeisaurus. These authors included Omeisaurus maoianus in addition to $O$. tianfuensis in their phylogenetic analyses, finding the former taxon to be more closely related to species of Mamenchisaurus than to $O$. tianfuensis. We found a similar result in the CEA-EW analysis, but in our preferred topologies, O. maoianus either branches earlier than Mamenchisauridae and $O$. tianfuensis (GEA-IW) or is in a clade in which Cetiosauriscus disrupts the monophyly of Omeisaurus (GEA-B). Regardless, these results collectively cast doubt on the monophyly of Omeisaurus. 
Finally, our preferred topologies agree with Xing et al. (2015) in finding M. youngi and the holotype of $M$. hochuanensis to be more closely related to each other than either is to Qijianglong (although this was not the case in the equal-weights parsimony analyses of the GEA and CEA matrices).

The largely discordant findings of these analyses, coupled with their agreement on the non-monophyly of Mamenchisaurus and Omeisaurus, emphasize the need for systematic revision of the anatomy and taxonomy of Middle-Late Jurassic East Asian sauropods. As noted by Xing et al. (2015), the first step should be a re-examination of the type species of Mamenchisaurus and Omeisaurus, as these species and their respective genera presently lack apomorphy-based diagnoses. Mamenchisaurus constructus, the type species of Mamenchisaurus, is among the most labile members of Euhelopodidae/Mamenchisauridae in our analyses, and is not recovered as closely allied with most other Middle-Late Jurassic Chinese sauropods in the CEA-IW analysis or in a small subset of MPTs of the GEA-EW and CEA-EW analyses. This lack of strong support for close kinship with other Middle-Late Jurassic Chinese eusauropods might be because of the limited character information that could be gleaned from the original description (Young 1954), or could foretell substantial revisions to the taxonomy of the group when more character information for $M$. constructus becomes available. Taken at face value, the sheer number of named sauropod taxa from the Middle-Late Jurassic strata of the Junggar and Sichuan basins of China - at least 14 genera, with six species of Mamenchisaurus and eight species of Omeisaurus - suggests that the species richness of this region rivals that of the Late Jurassic Morrison Formation of North America (Ikejiri 2005; Harris 2006; Tschopp et al. 2015a; Mannion et al. 2019), although the Chinese faunas are sampled over a longer time span. While some of these taxa might be subsumed into others following revision of the group, ideally through specimen-level phylogenetic analysis (Tschopp \& Upchurch 2019), we note that character score differences for all but the most incomplete taxa sampled here indicate substantial morphological, and presumably taxonomic, diversity.

Evolutionary relationships of Euhelopus. In the first published cladistic analysis of sauropods, Russell \& Zheng (1993) found Euhelopus to be more closely related to Brachiosaurus and Camarasaurus than to other Chinese sauropods, although the authors suggested that "links between the Chinese genera [Omeisaurus and Mamenchisaurus] and Euhelopus may be closer than suggested by this analysis" (Russell \& Zheng 1993, p. 2090). Analysis of the more comprehensive character matrices provided by Upchurch $(1995,1998)$ recovered Euhelopus outside of Neosauropoda in a clade with the Chinese taxa Shunosaurus, Omeisaurus and Mamenchisaurus, but subsequent cladistic analyses by Wilson \& Sereno (1998) and Wilson (2002) demonstrated that several of the characters that allied Euhelopus with these Chinese taxa in previous 
analyses were homoplastic, plesiomorphic for eusauropods, ambiguously distributed in sauropods or incorrectly scored, and provided character evidence that Euhelopus is instead an early branching somphospondylan. Upchurch et al. (2004) found Euhelopus, Mamenchisaurus and Omeisaurus as a paraphyletic grade of non-neosauropod eusauropods, but analysis of a rescored version of this matrix conducted as part of the redescription of Euhelopus by Wilson \& Upchurch (2009) recovered Euhelopus as a basally branching somphospondylan. Over the course of the last decade, consensus has emerged across phylogenetic analyses that Euhelopus is a non-titanosauriform macronarian (e.g. Royo-Torres et al. 2009; Carballido et al. 2011, 2017; Carballido \& Sander 2014; Lacovara et al. 2014; Gonzalez-Riga et al. 2016; Tykoski \& Fiorillo 2016; Britt et al. 2017; Fernandez-Baldor et al. 2017) or a somphospondylan (e.g. Curry Rogers 2005; You et al. 2008; Gonzalez Riga et al. 2009; Mannion 2010; Mateus et al. 2011; Sekiya 2011; Taylor et al. 2011; Zaher et al. 2011; D'Emic 2012, 2013; Royo-Torres et al. 2012, 2017; L€u et al. 2013; Mannion et al. 2013, 2017, 2019; Xing et al. 2015; R. D. F. Martınez et al. 2016; D'Emic et al. 2016; Gorscak \& O'Connor 2016; Poropat et al. 2016; Averianov et al. 2018; Gonzalez Riga et al. 2018).

The volume of studies cited above speaks to the substantial effort in recent years to document the anatomy and elucidate the evolutionary relationships of macronarian sauropods. Although the continued development of the CEA and GEA matrices and those of other authors has (until now) failed to perturb the position of Euhelopus within Macronaria, the severity of the test of non-macronarian affinities has arguably been limited by the lack of a commensurate effort to incorporate new observations on the anatomy of Middle-Late Jurassic Chinese eusauropods. That such observations should remain relevant to investigations of the evolutionary relationships of Euhelopus was demonstrated by Wilson \& Upchurch (2009), who found that a non-neosauropod position for Euhelopus could not be rejected by a Templeton test of the revised Upchurch et al. (2004) matrix analysed in that study. Nevertheless, some phylogenetic analyses of macronarian neosauropods conducted in the last 15 years have included Euhelopus without incorporating any early branching, non-neosauropod Chinese eusauropods (e.g. Curry Rogers 2005; Gorscak \& O’Connor 2016), or without including species of Mamenchisaurus (e.g. D'Emic 2012).

Unfortunately, the discovery of new character information pertinent to robust testing of the phylogenetic position of Euhelopus is greatly hampered by the generally poor representation of Middle-Late Jurassic Chinese sauropods in the literature. Although many taxa have been named (see above), most of this material has been insufficiently described and figured, preventing confident scoring of many characters - as, for example, with the original, brief description of Klamelisaurus by Zhao (1993). As a result, most phylogenetic studies that sample non-neosauropod eusauropods have relied principally on the comparatively rich and well-figured descriptions of Mamenchisaurus 
youngi (Ouyang \& Ye 2002) and Omeisaurus tianfuensis (He et al. 1988) to capture 'mamenchisaurid' morphology. However, although these monographs are critical sources of data for phylogenetic analyses, they do not capture the full anatomical diversity of Middle-Late Jurassic Chinese sauropods. Indeed, many of the features identified here as uniting Euhelopus with Klamelisaurus and other Core Mamenchisaurus-like Taxa are not present in Mamenchisaurus youngi or Omeisaurus tianfuensis, or cannot be confidently ascertained from the information presented in their respective publications.

Our focused study of Klamelisaurus and other Middle-Late Jurassic Chinese sauropods has revealed new character information that, in most of our phylogenetic analyses, supports a Euhelopodidae that includes Klamelisaurus and other Core Mamenchisaurus-like Taxa, although it is important to note that nodal support for this clade is poor in parsimony analyses, and topology tests of our parsimony results cannot reject alternative affinities for Euhelopus. In addition to the synapomorphies of a nonmacronarian Euhelopodidae and the node subtending Core Mamenchisaurus-like Taxa in the Bayesian analyses conducted here (Supplementary Tables 12, 13), Euhelopus shares numerous additional features with a subset of Core Mamenchisaurus-like Taxa (Supplementary Table 14). Below, we detail some of these traits.

Distolingual boss of the dentition. Noteworthy among the characters shared by some euhelopodids is the presence of a distinct boss on the distolingual surface of the teeth (Fig. 32). A rounded, boss-like structure on the lingual surface of the dentition has previously been considered an autapomorphy of Euhelopus (Wilson \& Sereno 1998; Wilson \& Upchurch 2009). We concur with Suteethorn et al. (2013) in noting the presence of an incipient boss on the distolingual surface of the dentary teeth of Mamenchisaurus sinocanadorum that is consistent with the placement and form of the boss in Euhelopus (Fig. 32). Although the holotype and only specimen of $M$.

sinocanadorum does not preserve the premaxilla or maxilla and thus exhibits the boss on the dentary teeth only, the presence of the distolingual boss in the teeth of the upper and lower jaws in Euhelopus (PMU 24705/1o; Wiman 1929, pl. I) suggests that this feature might also have been present in the upper jaw teeth of M. sinocanadorum.

The presence of a distolingual boss in isolated sauropod teeth has been invoked to indicate the presence of Euhelopus-like taxa outside the Mengyin Formation, in East Asia and in Spain during the Early Cretaceous (Canudo et al. 2002; Barrett \& Wang 2007; Suteethorn et al. 2013). Of particular significance among these instances is the presence of isolated teeth in the Lujiatun Beds of the Yixian Formation, which Barrett \& Wang (2007) referred to cf. Euhelopus. Two sauropods - Liaoningotitan (Zhou et al. 2018) and Dongbeititan (X. Wang et al. 2007) - have since been named from the Yixian Formation, both from the stratigraphically younger Jianshangou Beds. 
Liaoningotitan preserves teeth in the maxilla and dentary, but these were described as lacking the lingual convexity of Euhelopus (Zhou et al. 2018). The holotype of Dongbeititan does not preserve teeth, but the novel recovery in the GEA-EW and GEA-B analyses of Dongbeititan as a close relative of Euhelopus and bracketed by $M$. sinocanadorum predicts that Dongbeititan has teeth like those of Euhelopus, and suggests that the isolated teeth from the Lujiatun Beds may eventually be shown to be referable to Dongbeititan or a close relative. Notably, isolated teeth from a different locality of the Phu Kradung Formation than the site that yielded the Phu Kradung taxon also exhibit a low distolingual boss that is similar in form and position to those of Euhelopus and M. sinocanadorum (Suteethorn et al. 2013, fig. 6A-C). As with Dongbeititan, definitive referral of isolated teeth to other sauropod remains from the same formation cannot be made. Nevertheless, the recovery in most of our phylogenetic analyses of the Phu Kradung taxon as a relatively close relative of Euhelopus is significant, insofar as it provides another example in which isolated, Euhelopus-like teeth co-occur with skeletal material that phylogenetic analysis supports as being closely related to Euhelopus.

Our novel recovery of Dongbeititan in a non-macronarian Euhelopodidae (GEA-EW and GEA-B analyses) is supported by numerous features that it shares with Euhelopus and Core Mamenchisaurus-like Taxa, and deserves additional brief discussion, as previous studies have indicated that Dongbeititan is a macronarian. X. Wang et al. (2007) described Dongbeititan as a titanosauriform on the basis of camellate internal pneumatic tissue in the presacral vertebrae (also shared with Core Mamenchisauruslike Taxa and Euhelopus), pneumatopores in the dorsal ribs (also present in Qijianglong [Xing et al. 2015] and possibly O. tianfuensis [He et al. 1988, pl. VIII), strongly procoelous anterior caudal centra (present in Core Mamenchisaurus-like Taxa, turiasaurians and lithostrotians), and a medially deflected proximal end of the femur (present in some Core Mamenchisaurus-like Taxa and various eusauropods), and Dongbeititan has since been recovered in phylogenetic analyses as a somphospondylan (e.g. Mannion \& Calvo 2011; D'Emic 2012; Mannion et al. 2013, 2017; Poropat et al. 2015, 2016; Gonzalez Riga et al. 2018) or non-titanosaurian macronarian (Mannion et al. 2013). Dongbeititan shares several additional features with Core Mamenchisauruslike Taxa that otherwise have a relatively restricted distribution among eusauropods, including an increase in cervical number to at least 17 (otherwise definitively present only in Core Mamenchisaurus-like Taxa, Euhelopus and Rapetosaurus), an anterior margin of the neural spine in posterior dorsal vertebrae that is level with or posterior to the posterior margin of the neural arch (X. Wang et al. 2007, fig 1B; an exclusive synapomorphy of the node subtending Core Mamenchisaurus-like Taxa, secondarily lost in Euhelopus and Daxiatitan in the GEA-B and CEA-B analyses; Supplementary Table 13), a medially deflected scapular glenoid (also present in Klamelisaurus), and a 
tibial condyle of the femur that is at least 1.2 times as long anteroposteriorly as the fibular condyle (present in Klamelisaurus, Euhelopus and Daxiatitan).

Robustly developed anterior epipophyseal muscle scar. A rugose anterior epipophyseal muscle scar that extends anteriorly into the SDF, giving the fossa a scalloped posterior margin, was previously described as the EPRL, and is present in Klamelisaurus, Euhelopus and some other sauropods. We discuss the homology and distribution of this feature at length below (see Homology of the 'epipophysealprezygapophyseal lamina').

Ventrally convex PRDL in middle and posterior cervical vertebrae. Euhelopus, Klamelisaurus and Qijianglong exhibit a ventrally convex PRDL in middle and posterior cervical vertebrae. A more tab-like or discrete bulge in the contour of the PRDL is present in Tienshanosaurus (Young 1937, fig. 1B), Shunosaurus (Y. Zhang 1988, fig. 26), some cervical vertebrae of Bellusaurus (IVPP V17768) and some posterior cervical vertebrae of Qijianglong (QJGPM 1001; Xing et al. 2015, fig. 12B, F). Given the presence of both types of interruption of the margin of the PRDL in Qijianglong, we score these morphologies as a single character. Interestingly, convexity of the middle-posterior cervical PRDL is apparently limited to a subset of Chinese sauropods, but these taxa do not form a monophyletic group in any of our analyses.

Ventrally bifurcate PODL in posterior-most cervical vertebrae. This character appears to be unique to Klamelisaurus, Euhelopus, an isolated posterior cervical vertebra from the Phu Kradung Formation of Thailand and the 'Shishugou cervicodorsal vertebrae' (Figs 6A, C, 33). A ventrally bifurcate PODL is strongly developed in the anterior-most of the three Shishugou cervicodorsal vertebrae, wherein two distinct rami extend onto the posterodorsal and posterior aspects of the diapophysis (Fig. 33D). Though not described by Suteethorn et al. (2013), a ventrally bifurcate PODL is also well developed on the left side of a posterior cervical vertebra from the Phu Kradung Formation referred to Mamenchisaurus sp. (Fig. 33C). Bifurcation of the PODL is less robustly developed in Klamelisaurus and Euhelopus, but is nonetheless clearly present. In Klamelisaurus, a ventrally bifurcated PODL is distinct on the left side of cervicals 15 and 16, but is absent on the right side of cervical 15 and indeterminate on the right side of cervical 16 (Fig. 6A, C). As in Klamelisaurus, PODL bifurcation in Euhelopus exhibits left-right asymmetry: the PODL is split on the right but not the left side of cervical 15, is unsplit on the right side of cervical 16 but shallowly bifurcate on the left side, and is shallowly bifurcated on both sides of cervical 17 (Fig. 33A, B; Wiman 1929, pl. III; Wilson \& Upchurch 2009, figs 11, 12). Although a ventrally bifurcate PODL was not mentioned by Wilson \& Upchurch (2009) or Suteethorn et al. (2013), this subtle feature of the posterior-most cervical 
vertebrae consistently supports a subclade of Mamenchisauridae/Euhelopodidae in our phylogenetic analyses, highlighting the importance of reporting small serial variations in vertebral pneumatization and lamina conformation.

That these sorts of features may be serially and symmetrically variable does not justify their exclusion from specimen-based phylogenetic analysis (Tschopp \& Upchurch 2019). Asymmetry and serial variation are pervasive in the development of sauropod vertebral laminae (Wilson 1999, 2012; Tschopp \& Upchurch 2019). Numerous examples of such variation are described in this study, including ventral bifurcation of the PODL in the posterior-most cervical vertebrae of Euhelopus and some Core Mamenchisaurus-like Taxa, expression of the PCPL in posterior dorsal vertebrae of Klamelisaurus, and the development of accessory struts of the SDF in the cervical vertebrae of various sauropods. Asymmetry of sauropod vertebral laminae and other structures influenced by pneumatic epithelia probably reflects the opportunistic pneumatization of bone by diverticula of the respiratory system; such asymmetry is expected to be particularly pronounced in the early evolutionary history of these structures (Wedel \& Taylor 2013b). Thus, ignoring repeated instantiations of otherwise uncommon pneumatic features or scoring them as polymorphic (' $0 / 1^{\prime}$ ') or ambiguous ('?') because they are asymmetric risks eliminating phylogenetically informative observations (Tschopp \& Upchurch 2019).

Fourth femoral trochanter positioned near midline of posterior surface. The position of the femoral fourth trochanter has a complex distribution near the origins of Sauropoda (McPhee \& Choiniere 2017). Plesiomorphically for sauropodomorphs, the fourth trochanter is positioned on or near the midline of the posterior surface of the femur, but around the base of Sauropoda several taxa exhibit a medially shifted fourth trochanter, including Antetonitrus (Yates \& Kitching 2003; McPhee et al. 2014), Lessemsaurus (Pol \& Powell 2007), Isanosaurus (Buffetaut et al. 2000), Vulcanodon (Raath 1972; Cooper 1984) and Tazoudasaurus (Allain \& Aquesbi 2008), and this condition is maintained in most eusauropods (Fig. 34). However, Patagosaurus (Bonaparte 1986a), Spinophorosaurus (Remes et al. 2009), Shunosaurus (Y. Zhang 1988), some specimens of Bellusaurus (IVPP V17768), Klamelisaurus, Euhelopus (PMU 224), Mamenchisaurus youngi (Ouyang \& Ye 2002) and Oceanotitan (Mocho et al. 2019) all exhibit the plesiomorphic condition of having the fourth trochanter positioned at or near the midline of the femur (Fig. 34A-E). One possible explanation for these apparent reversals to a medially positioned fourth trochanter among nonneosauropod eusauropods is that they reflect an interval of conserved developmental plasticity - a 'zone of variability' (Bever et al. 2011) - in the insertion site of $\mathrm{m}$. caudofemoralis musculature, with population-level polymorphism in the position of the fourth trochanter maintained across several speciation events. The latter interpretation might garner support from the observation that the placement of the fourth trochanter 
in Bellusaurus is variable: the fourth trochanter lies on the posterior midline in IVPP V17768-62 (Fig. 34E), while in IVPP V17768-276, the fourth trochanter nearly abuts the medial margin (Mo 2013, pl. 59; note that anteroposterior crushing of this specimen positions the fourth trochanter more laterally than it would have been in life). Whatever the reason for the substantial homoplasy in this character, a fourth trochanter positioned near the femoral midline supports a clade comprising Euhelopus, Klamelisaurus and Mamenchisaurus youngi in all analyses of the CEA matrix.

\section{The evolutionary relationships of Bellusaurus and insights into the ontogeny of} postcranial skeletal pneumaticity. Bellusaurus sui is an enigmatic eusauropod that, like Klamelisaurus, Tienshanosaurus and Mamenchisaurus sinocanadorum, is known from the Middle-Late Jurassic Shishugou Formation. Bellusaurus is of particular importance for studies of Shishugou Formation sauropods because all known Bellusaurus material is juvenile - as evidenced by gross skeletochronological indicators (e.g. unfused neurocentral sutures and porous, striated bone texture) and osteohistological evidence indicating death within the first 2 years post-hatching (Dong 1990; Mo 2013; Moore et al. 2018) - and could therefore represent an earlier ontogenetic stage of another named Shishugou Formation taxon. It was presumably on these grounds that Paul (2010) hypothesized that Klamelisaurus is the adult form of Bellusaurus, but the limited anatomical information available for Klamelisaurus in the literature prior to this study has prevented explicit consideration of this hypothesis.

Bellusaurus lacks the autapomorphies identified in Klamelisaurus, and the weight of evidence from our anatomical comparisons (see Differential diagnosis, above) and phylogenetic analyses indicates that Klamelisaurus and Bellusaurus are distinct and relatively distantly related eusauropods. Nevertheless, Bellusaurus shares a number of features with Klamelisaurus and other Core Mamenchisaurus-like Taxa that support close kinship of these sauropods in the CEA-IW analysis, highlighting the need for additional data on the anatomy and ontogeny of Middle-Late Jurassic East Asian eusauropods.

The phylogenetic position of Bellusaurus has been uncertain since its original, brief description by Dong (1990). Recent phylogenetic analyses including Bellusaurus have agreed that the taxon lies just outside Neosauropoda (Wilson \& Upchurch 2009; RoyoTorres \& Upchurch 2012; Mo 2013; Mannion et al. 2019) or near the base of Macronaria (Upchurch et al. 2004; Carballido \& Sander 2014; Royo-Torres et al. 2017), and several cranial features supporting a position in or near Neosauropoda were recently identified (Moore et al. 2018). Some of our phylogenetic analyses are broadly consistent with previous work in finding Bellusaurus to be either the sister taxon of Neosauropoda (GEA-B) or an early branching constituent of Macronaria (all analyses of the CEA matrix). 
However, both equal and extended implied-weights parsimony analysis of the GEA matrix recover Bellusaurus in a novel position within Diplodocoidea, as sister to Diplodocimorpha, which would make Bellusaurus the second diplodocoid known from East Asia (X. Xu et al. 2018). This finding is supported by numerous features from across the skeleton, including proatlantal facets on the otoccipital (also present in turiasaurians and some titanosaurians), the lack of a foramen or foramina between the basal tubera and basipterygoid processes (a condition also exhibited by some titanosaurians), posterior dorsal neural arches with steeply orientated postzygapophyses (also present in Tehuelchesaurus and some somphospondylans), and a fibular facet of the astragalus that faces posterolaterally (unique among sauropods to Haplocanthosaurus, Bellusaurus and Diplodocoidea: Whitlock 2011; Tschopp et al. 2015a). A close affinity to diplodocoids might be supported by features not included in one or both matrices analysed here, including a vertical strut or struts within the lateral pneumatic foramen of the dorsal centra (present in various diplodocoids, including Katepensaurus, Dinheirosaurus, Supersaurus and Diplodocus, as well as a few macronarians), a lateral branch of the CPOL in middle and posterior dorsal neural arches (present in various flagellicaudatans as well as Losillasaurus, Giraffatitan and Camarasaurus; Tschopp et al. 2015a) and a well-developed ambiens process of the pubis (present in various flagellicaudatans: Tschopp et al. 2015a). In addition, Bellusaurus shares with numerous flagellicaudatans, including the recently described Middle Jurassic Chinese dicraeosaurid Lingwulong, a squamosal with a posterior/ posteroventral process that buttresses the paroccipital process (Moore et al. 2018; X. $\mathrm{Xu}$ et al. 2018).

Although most of our phylogenetic analyses find Bellusaurus to be only distantly related to Klamelisaurus and other Core Mamenchisaurus-like Taxa, Bellusaurus is recovered as an early branching member of a macronarian Euhelopodidae in the CEAIW analysis. Such a close relationship with Core Mamenchisaurus-like Taxa is unique to this study and is supported by several features (e.g. posteriorly projecting, prong-like epipophyses in the cervical vertebrae; a PRDL of the middle-posterior cervical vertebrae interrupted by a bulging projection; pleurocoels of anterior and middle dorsal centra with acute posterior margins; procoelous anterior-most caudal vertebrae; at least one middle caudal vertebra with a neural spine that doubles in anteroposterior length dorsally), although with the exception of a bulging projection of the PRDL and anteroposteriorly expanded middle caudal neural spines, these features are also present in other eusauropods. Bellusaurus also shares with Klamelisaurus a few peculiar features that are otherwise absent or uncommon among sauropods: both exhibit a dorsally bifurcate SPDL in middle and posterior dorsal vertebrae (considered an autapomorphy of Bellusaurus by Mo [2013], but also present in Moabosaurus, Mierasaurus and some titanosaurians; Poropat et al. 2016; Mannion et al. 2019), and 
have two distinct ridges on the proximal, anterior face of anterior dorsal ribs (Fig. 14), although the distribution of this feature in sauropods is unclear. In addition, the presence of dorsolaterally flattened and transversely expanded SPRLs in some middleposterior cervical vertebrae of Bellusaurus could represent nascent development of the sheet-like muscle insertions identified here as autapomorphic for Klamelisaurus. It is also noteworthy that Bellusaurus exhibits denticles on dentary teeth only, reminiscent of the distribution of denticles in Mamenchisaurus sinocanadorum and M. youngi (see above). Considered together, these observations and the results of the CEA-IW analysis indicate that we cannot exclude the possibility that Bellusaurus is a juvenile euhelopodid/mamenchisaurid.

Hypothesizing that Bellusaurus represents the juvenile form of Klamelisaurus (Paul 2010) or a close relative implies a large suite of ontogenetic transformations, many of which concern pneumatic features of the axial skeleton. Despite a general dearth of single-taxon ontogenetic series of sauropods, several studies over the last two decades have offered considerable insight into ontogenetic variation in sauropod postcranial skeletal pneumaticity (Wedel 2003; Schwarz et al. 2007; Carballido \& Sander 2014; Melstrom et al. 2016).

The most robustly established ontogenetic trend in postcranial skeletal pneumaticity is one of increasing invasiveness and ramification of pneumatic air spaces throughout development. Empirical observations in sauropods and theoretical extrapolations from the available data indicate that camerate and camellate architecture can be derived from acamerate and procamerate conditions, although for camellate taxa, described ontogenetic series are scarce and represent end points on a pneumatic continuum, leaving open the question of how, exactly, camellate morphologies derive from procamerate precursors (Wedel 2003; Schwarz et al. 2007; Carballido \& Sander 2014; Melstrom et al. 2016).

An ontogenetic link between Bellusaurus and mature exemplars of Klamelisaurus or other Core Mamenchisaurus-like Taxa requires that pneumatic structures of the latter taxa could have developed from morphologies present in Bellusaurus. Unfortunately, the internal anatomy of Klamelisaurus is not discernible from external examination of the lateral pneumatic excavations of its centra and is obscured or unclear at broken surfaces of the vertebrae. However, closely related taxa with similar external morphology have been described as exhibiting camellate internal architecture (Young \& Zhao 1972; Russell \& Zheng 1993; Ouyang \& Ye 2002), and euhelopodids/ mamenchisaurids evolved camellate pneumaticity in their centra independently of titanosauriforms (Wedel et al. 2000; Wedel 2003). Given this, it is reasonable to hypothesize that Klamelisaurus bears internal pneumatic architecture similar to that of closely related taxa. 
Many of the referred cervical centra of Bellusaurus (IVPP V17768) exhibit procamerate, or perhaps weakly camerate, conditions (sensu Wedel et al. 2000; Fig. $35 \mathrm{~A}, \mathrm{~B})$. Lateral pneumatic excavations of the centra are generally deep, leaving only a thin midline septum, and are often sharp-lipped throughout their circumference. Pneumatic excavations occupy at least half of the lateral surface of the centrum (exclusive of the anterior condyle), although many exceed more than two-thirds the length of the centrum; internally, pneumatic chambers do not extend appreciably into the anterior condyle. Nearly all of the excavations are fully subdivided by an oblique septum, and several elements have an additional subparallel or orthogonal strut that crosses this excavation (Fig. 35A, B).

The cervical centra of Bellusaurus exhibit a degree of pneumatization distinct from that described for very early stage juvenile neosauropod centra, which show weakly procamerate or ambiguously pneumatic fossae that generally lack subdividing septa (Carpenter \& McIntosh 1994; Wedel et al. 2000; Carballido \& Sander 2014). Bellusaurus is instead more similar to SMA 0009, a juvenile brachiosaurid (Schwarz et al. 2007; Carballido et al. 2012), and a recently described juvenile Barosaurus (Melstrom et al. 2016) in exhibiting nascent subdivision of the lateral pneumatic excavation. Like some Bellusaurus specimens, the preserved cervical vertebrae of the juvenile Barosaurus bear one or two oblique, subparallel laminae that subdivide a welldeveloped, procamerate lateral pneumatic chamber, the outer margins of which occupy at least half the length of the centrum (Melstrom et al. 2016). Adult Barosaurus develop an irregular, camellate internal architecture (Lull 1919; Taylor \& Wedel 2013), thus bearing out the hypothesis that camellate pneumatic architecture is presaged ontogenetically by relatively large, simple chambers, which ramify as vertebrae are remodelled through ontogeny (Wedel 2003).

These observations suggest that more mature individuals of Bellusaurus might have had the capacity to develop an internal cervical architecture as complex as that of Barosaurus, although at present we cannot rule out the possibility that adult Bellusaurus exhibited a less complicated camerate, or polycamerate, structure. Pneumaticity in Bellusaurus cervical centra thus fails to contradict the hypothesis that Bellusaurus is a juvenile euhelopodid/mamenchisaurid (contra Moore et al. 2018), but remains consistent with evolutionary hypotheses that place Bellusaurus as an early branching diplodocoid or macronarian, because both lineages exhibit cervical pneumaticity that is more complex than the procamerate condition in taxa close to the origin of these clades.

Although pneumatic morphologies of the centrum do not contradict the possibility that Bellusaurus is a juvenile euhelopodid/mamenchisaurid, several striking morphologies of the dorsal series of Bellusaurus do contradict this hypothesis. Bellusaurus exhibits 
several apparently pneumatic features of the dorsal neural arches and spines that are absent in Klamelisaurus and its closest relatives, a condition contrary to that expected if Bellusaurus were a juvenile euhelopodid/mamenchisaurid (Wedel 2003; Carballido $\&$ Sander 2014). We present three examples to illustrate this point, two of which were mentioned in the description and comparison above. First, Bellusaurus exhibits a ventrally bifurcate PCDL in middle and posterior dorsal vertebrae (Fig. 13B), a feature most common in titanosaurians but also occurring in a few non-titanosauriform eusauropods, including Tehuelchesaurus (Salgado et al. 1997; Carballido et al. 2011; Mannion et al. 2013). Second, Bellusaurus has a sharp-edged lateral branch of the $\mathrm{CPOL}$ in posterior dorsal vertebrae that is recessed within a relatively deep fossa on the posterior aspect of the diapophysis (Fig. 13B); in contrast, this feature is only weakly developed in Klamelisaurus (Fig. 13A). Lastly, unlike Core Mamenchisaurus-like Taxa, the diapophyses of at least some posterior dorsal vertebrae of Bellusaurus bear longitudinal, trough-like depressions with distinct rims on their dorsal surfaces (Fig. $35 \mathrm{C}, \mathrm{D})$. This morphology corresponds closely to that observed in posterior dorsal vertebrae of Europasaurus (Carballido \& Sander 2014) (Figs 21D, 22D), and is evocative of the dorsal fossae of the diapophyses of anterior dorsal vertebrae in Tendaguria (Mannion et al. 2019) and of the laterodiapophyseal fossa/fenestra of the rebbachisaurid Katepensaurus, the latter being almost certainly pneumatic in origin (Ibiricu et al. 2013, 2015, 2017).

In summary, while the weight of character evidence across the phylogenetic analyses presented here indicates that Bellusaurus is a neosauropod (or close relative) that is only distantly related to Klamelisaurus, some conspicuous similarities between Bellusaurus, Klamelisaurus and other euhelopodids/mamenchisaurids suggest that future analyses might favour closer kinship between Bellusaurus and Core Mamenchisaurus-like Taxa. Robust resolution of the evolutionary affinities of Bellusaurus remains complicated by a host of factors. Most obvious of these is the difficulty of identifying ontogenetically variable characters, and whether and how to account for such variability in phylogenetic analyses (see discussion in Moore et al. 2018). Some studies have suggested that juvenile specimens may slip stem-ward if they lack apomorphies that are acquired during ontogeny (Kammerer 2011; Campione et al. 2013; Choiniere et al. 2013; Carballido \& Sander 2014; Tsai \& Fordyce 2014; Currie et al. 2016; S. Wang et al. 2017). However, juveniles of lineages with convergently evolved features - such as the camellate internal architecture of cervical vertebrae in euhelopodids/mamenchisaurids, titanosauriforms and Barosaurus - might slip to the stem of other lineages if unrelated character evidence does not anchor them to their true lineage; such would be the case for our phylogenetic analyses, if Bellusaurus is eventually shown to be a member of a non-neosauropod Euhelopodidae/ Mamenchisauridae. Although we have documented numerous characteristics of 
Bellusaurus that appear to be lacking in penecontemporaneous Chinese sauropods, including seemingly derived instances of neural arch pneumatization in dorsal vertebrae, in the absence of comprehensive descriptions for the vast majority of Middle-Late Jurassic Chinese taxa, the true distribution of these features remains uncertain. Ultimately, future progress will require sustained efforts to comprehensively document the anatomy of Jurassic Chinese sauropods, new discoveries of immature sauropods, and continual re-evaluation of the phylogenetic affinities of Bellusaurus as new information comes to light.

\section{Implications for sauropod palaeobiogeography}

The presence of apparently endemic lineages of Chinese eusauropods and other Mesozoic vertebrate groups has been suggested to reflect geographical isolation of East Asia during the Middle-Late Jurassic, principally by the establishment of the Russian Sea as a north-south marine barrier between eastern Europe and Central Asia (Russell 1993; Upchurch 1995; Barrett et al. 2002; Wilson \& Upchurch 2009; Poropat et al. 2016; X. Xu et al. 2018). Whereas early proponents of the hypothesis suggested that the period of isolation extended into the Early Cretaceous (Russell 1993; Barrett et al. 2002), a recent review of palaeogeographical evidence for dispersal barriers limits the window of East Asian isolation to the late Callovian-early Kimmeridgian (X. Xu et al. 2018).

The recent discovery of the dicraeosaurid Lingwulong from the Middle Jurassic of north-central China (X. Xu et al.2018) and the recovery of Bellusaurus as an early branching neosauropod (Upchurch et al. 2004; Carballido \& Sander 2014; Royo-Torres et al. 2017; Moore et al. 2018; this study) indicate that diplodocoids, and perhaps macronarians, were present in East Asia from the early Middle Jurassic onwards, with their low abundance in palaeontological collections perhaps reflecting biases in spatial and temporal sampling (X. Xu et al. 2018). Although multiple instances of dispersal and isolation can provide more, not less, support for hypotheses of isolation and endemism (Wilson \& Upchurch 2009), it is nonetheless apparent that the supposed distinctiveness of Middle-Late Jurassic East Asian sauropod faunas might not have been as great as thought previously.

The recovery of the non-East Asian taxa Wamweracaudia and Cetiosauriscus as nested members of Mamenchisauridae/Euhelopodidae in some or all of our phylogenetic analyses provides palaeobiogeographical support for the hypothesis that the separation between East Asia and the rest of Pangaea was not absolute throughout the MiddleLate Jurassic, and that the window of palaeogeographical isolation might have been limited to the Callovian-early Kimmeridgian (X. Xu et al. 2018). Cetiosauriscus stewarti is from the Callovian Oxford Clay of England, while Wamweracaudia keranjei 
was recovered from the Tithonian (or possibly Berriasian) Tendaguru Formation of Tanzania (Bussert et al. 2009; Mannion et al. 2019). The placement of these taxa within a clade otherwise composed entirely of Asian forms implies the presence of dispersal routes out of East Asia prior to the onset of isolation in the Callovian, and could be consistent with their re-establishment by the Tithonian. However, given the probable separation of Laurasia from Gondwana beginning in the Callovian (Poropat et al. 2016; Mannion et al. 2019), it is more likely that the presence of Wamweracaudia in Africa resulted from pre-isolation emigration of this lineage out of East Asia. Importantly, our results, and recent palaeobiogeographical analyses that include Wamweracaudia (Mannion et al. 2019), undermine the hypothesis that euhelopodids/mamenchisaurids constitute an endemic radiation of non-neosauropod eusauropods restricted to East Asia. These findings also leave open the possibility that euhelopodids/ mamenchisaurids, like other non-neosauropod eusauropods, were more broadly distributed across much of Pangaea in the Middle Jurassic, with sampling biases in the fossil record and regional extinctions masking the signal of this earlier distribution (X. $\mathrm{Xu}$ et al. 2018; Mannion et al. 2019).

The stratigraphic range of non-neosauropod East Asian eusauropods Bayesian analyses of the GEA and CEA matrices predict the origination of a nonmacronarian Euhelopodidae in the latter half of the Early Jurassic or earliest Middle Jurassic, with the $95 \%$ highest posterior density on the age of the node concentrated in the late Sinemurian, the Pliensbachian and the first half of the Toarcian for the GEA matrix (179.9-193.7Ma) and the late Pliensbachian through the earliest Aalenian for the CEA matrix (173.3-184.0 Ma). Chuanjiesaurus, from the Middle Jurassic Chuanjie Formation (Fang et al. 2000), constitutes the oldest body fossil record for the group and conservatively implies an origin by the Aalenian. Recovery of Euhelopus, Dongbeititan, Daxiatitan and (in the GEA-B analysis) Xianshanosaurus within a nonmacronarian Euhelopodidae extends the stratigraphic range of non-neosauropod eusauropods well into the Early Cretaceous. Until recently, such a phylogenetic position, and age estimates based largely on vertebrate biostratigraphy, would have implied a chronostratigraphic gap between Euhelopus (the oldest of these Early Cretaceous taxa) and Core Mamenchisaurus-like Taxa of the Sichuan and Junggar basins of up to $\sim 50$ million years (using, for example, the age ranges provided for many of the relevant formations in Weishampel et al. [2004]); however, recent radiometric studies have closed this gap substantially (see below). These studies, and the phylogenetic results presented here, provide evidence for the persistence of euhelopodids/ mamenchisaurids into the Early Cretaceous, contradicting inferences by previous authors that no non-neosauropod lineages survived across the JurassicCretaceous boundary (Mannion et al. 2011, 2013; Xing et al. 2015). 
The Mengyin Formation, from which Euhelopus was recovered, has historically been considered Late Jurassic (early Tithonian) to Early Cretaceous in age (as late as Aptian; see review in Wilson \& Upchurch 2009). Uncertainty in the age of the Mengyin Formation was recently narrowed by a stratigraphic series of non-conflicting detrital zircon ages from the Luxi Basin. These data indicate that the Mengyin Formation is 144-130.7Ma (J. Xu \& Li 2015), constraining the age of Euhelopus to the BerriasianHauterivian. Less extreme uncertainty in age has typified studies of the Shaximiao Formation, which has been considered Middle-Late Jurassic (Dong et al. 1983; Russell 1993) or wholly Middle Jurassic in age (Li et al. 2011; Xing et al. 2015). Recent radiometric dating of detrital zircons from beds of the lower Shaximiao Formation at Dashanpu resolves much of this uncertainty, providing an age of $159 \pm 2 \mathrm{Ma}$ (based on recalculations of the ages of the zircons with $10 \%$ discordance and 1 standard deviation error; J. Stiegler, pers. comm., 2018) and placing the entirety of the Shaximiao Formation within the Late Jurassic (J. Wang et al. 2018; see also Y. Li et al. [2018] for concordant age estimates for the lower Shaximiao Formation; Supplementary Table 9). These results imply that taxa from the upper Shaximiao Formation could be as young as Tithonian in age. This shift in taxic diversity of the Shaximiao Formation from the Callovian to the Oxfordian, as well as radiometric dates placing most of the Shishugou Formation in the Oxfordian (Choiniere et al. 2013; Han et al. 2016), might dampen the severity of the apparent crash in Oxfordian sauropod diversity inferred by previous studies (Upchurch \& Barrett 2005; Barrett et al. 2009; Mannion et al. 2011).

Confirmation via phylogenetic analysis that an isolated posterior cervical vertebra from the Phu Kradung Formation (Suteethorn et al. 2013) is closely related to species of Mamenchisaurus indicates that the lineage survived at least until the JurassicCretaceous boundary, as the Phu Kradung Formation is considered to be latest Jurassic or earliest Cretaceous in age on the basis of vertebrate biostratigraphy and palynological evidence (Racey \& Goodall 2009; Suteethorn et al. 2013). In addition, the Suining Formation, which overlies the Shaximiao Formation and has yielded Qijianglong and M. anyuensis (He et al. 1996), was recently found to be as young as Aptian in age based on U-Pb radiometric dating of detrital zircons (Liu et al. 2017; J. Wang et al. 2019); these dates imply substantial extension of Mamenchisaurus-like taxa into the Early Cretaceous, regardless of the phylogenetic affinities of Euhelopus. Taken in sum, these data close the implied chronostratigraphic gap between Euhelopus and Core Mamenchisaurus-like Taxa and indicate that, even if Euhelopus, Daxiatitan, Dongbeititan and Xianshanosaurus are considered titanosauriforms, East Asian nonneosauropods survived into the Early Cretaceous (J. Wang et al. 2019), although more research on the phylogenetic affinities of taxa from the Suining Formation and overlying Penglaizhen Formation is needed. In concert with the recovery of the Early Cretaceous Mierasaurus and Moabosaurus in a non-neosauropod Turiasauria (Royo- 
Torres et al. 2017; Mannion et al. 2019; GEA-IW, GEA-B, CEA-EW and CEA-B analyses), this finding suggests that two distinct lineages of non-neosauropods survived across the Jurassic-Cretaceous boundary.

Homology of the 'epipophyseal-prezygapophyseal lamina' (EPRL)

In saurischians and other sauropsids, the lateral surface of the cervical neural arch may be crossed by a horizontally orientated ridge or ridges of variable prominence. In various squamates, a 'postzygoprezygapophyseal lamina' spans the interval between the pre- and postzygapophyses (Tschopp 2016). Some saurischians with large cervical epipophyses develop a lamina that crosses the SDF, linking the epipophysis and prezygapophysis (Wilson 2012). Among non-avian theropods, this lamina is restricted to ceratosaurians and is well developed in Carnotaurus (Bonaparte 1996, 1986b; Mendez 2014, figs 4-9), Majungasaurus (O'Connor 2007, figs 7, 8) and Dahalokely (Farke \& Sertich 2013, figs 2, 3), where a smooth, sharp lamina demarcates the dorsal and lateral surfaces of the neural arch. Among sauropods, a similar feature was first noted in Nigersaurus, where it was figured in the fifth cervical vertebra and termed the 'prezygapophyseal-epipophyseal lamina' (Sereno et al. 2007, fig. 3). In accord with the nomenclature devised by Wilson (1999), this strut was renamed the epipophysealprezygapophyseal lamina (EPRL) by Wilson \& Upchurch (2009), who identified its presence throughout the cervical series as an autapomorphy of Euhelopus, convergently present in Nigersaurus, several other sauropods and some theropods. In Nigersaurus MNBH GAD 512 and some other sauropods, the EPRL does not reach either the epipophysis or the prezygapophysis directly, but is considered to be connected to these landmarks via its union with the SPRL and PODL (Wilson et al. 2011).

In posterior cervical vertebrae of Klamelisaurus, two topologically and morphologically distinct structures on the lateral side of the neural arch are potentially interpretable as an EPRL. The first is a rugose anterior extension of the epipophysis that invades the SDF posteriorly. Because it is continuous with the epipophysis but well removed from the prezygapophysis and SPRL, the ridge might be called a 'stranded epipophyseal lamina' (Wilson 2012). This is the interpretation made by Wilson (2012) for a similar ridge in Uberabatitan, and an identically situated rugosity in Euhelopus was considered to constitute a portion of the EPRL by Wilson \& Upchurch (2009, figs 9,11). The second feature that could be considered an EPRL in Klamelisaurus is a horizontal strut within the SDF that is not attached to surrounding landmarks (epipophysis, prezygapophysis) or laminae (PODL, SPRL). Such an unmoored horizontal strut of the SDF has previously been interpreted to constitute an incipient EPRL in taxa such as Mamenchisaurus, Camarasaurus, Limaysaurus and Apatosaurus 
louisae (Wilson et al. 2011; Tschopp et al. 2015a, character 187), and is similar in position to the well-developed strut that fully connects the SPRL and PODL in Nigersaurus.

It is possible to simultaneously interpret both of these features in Klamelisaurus as a form of EPRL by invoking the concept of 'segmented laminae'. The possibility that a lamina might be segmented was proposed by Salgado \& de Souza Carvalho (2008), who described the apparently novel segmentation of the PODL in anterior and middle cervical vertebrae of Uberabatitan into zygapophyseal and diapophyseal components. Wilson (2012) instead interpreted Uberabatitan, as well as the 'Series A' titanosaurian (Powell 1987, 2003), as having stranded serial variants of two different laminae: a stranded epipophyseal lamina (a variant of the EPRL) and a stranded diapophyseal lamina (a variant of the PODL). Wilson (2012) noted, however, that truly segmented laminae - those in which separate, smaller segments can be recombined to form the original lamina - do occur, as in the case of the segmentation in anterior dorsal vertebrae of the ACDL into the ACPL and PPDL by the parapophysis. Although they have not been explicitly described as such, the anterior and middle cervical vertebrae of some non-sauropod sauropodomorphs (e.g. Sarahsaurus, Massospondylus) appear to exhibit a segmented EPRL, with separate, overlapping epipophyseal and prezygapophyseal components that, at least in some posterior cervical vertebrae, combine to make a single, broad ridge (TMM 43646-2; TMM 43646-3; BP/1/4934; A. D. Marsh \& Rowe 2018; Barrett et al. 2019; see below).

Because both a rugose anterior extension of the epipophysis and an unmoored strut of the SDF have previously been interpreted as an EPRL, the joint presence of these structures in middle and posterior cervical vertebrae of Klamelisaurus might indicate that the EPRL is segmented. This interpretation is, however, unsatisfactory. As we elaborate below, the two types of ridges observed in Klamelisaurus are inferred to have resulted from the morphogenetic influence of distinct soft tissues, with the rugose extension of the epipophysis produced by muscle attachments and the horizontal strut of the SDF associated with pneumatizing epithelium. Although sauropod vertebral laminae undoubtedly served as attachments for muscles - the crista-transverso-obliqua (=SPOL) of extant birds, for example, is an insertion for portions of the $\mathrm{mm}$. intercristales (Wedel \& Sanders 2002; Tsuihiji 2005) - the observed partitioning of the 'segmented EPRL' of Klamelisaurus into what can plausibly be interpreted as separate muscular and pneumatic parts suggests that these structures are more informatively considered distinct, rather than co-dependent, entities. As we show below, the distinctiveness of these structures is evidenced in other sauropod taxa. Camarasaurus, Euhelopus and Nigersaurus can also be interpreted as having a 'segmented EPRL' composed of muscular and pneumatic parts. In addition, a pronounced anterior extension of the epipophysis can be present in the absence of a strut of the SDF (e.g. 
Camarasaurus - BYU 9047; Uberabatitan - Silva Junior et al. 2019, figs 3, 4), and struts of the SDF may be present without a marked anterior epipophyseal ridge (e.g. Apatosaurus louisae - Gilmore 1936, pl. 24, Tschopp et al. 2015a; Galeamopus Tschopp \& Mateus 2017, fig. 27).

The great advantage of the nomenclature for vertebral laminae devised by Wilson (1999) and elaborated in subsequent studies (Wilson et al. 2011; Wilson 2012) is that its straightforward reliance on the connectivity of laminae to vertebral landmarks allows for the objective and repeatable recognition of primary homologues (Pinna 1991), the historical relationships of which can be subsequently tested by phylogenetic analysis (Wilson 1999, 2012). In the limited case of the EPRL, however, the position of struts or ridges on the lateral surface of the sauropodomorph cervical neural arch, in the absence of other evidence for assessing similarity (Patterson 1982; Rieppel \& Kearny 2002; Simões et al. 2017), is often an insufficient basis for assertions of primary homology, because these morphologies can be plausibly interpreted to have arisen from the influence of distinct soft tissues. Ignoring this possibility allows for erroneous assertions of primary homology, which in turn undermine the clarity of descriptive anatomical work and have the potential to bias phylogenetic analysis.

That phylogenetic analyses may be affected by a lack of clarity surrounding recognition of the EPRL is evidenced by the widely different scoring of characters concerning the EPRL in two recent phylogenetic analyses of eusauropods. Using an expanded version of the GEA matrix, Mannion et al. (2019, character 129) found a cervical EPRL to be sparsely and homoplastically distributed among eusauropods, present only in Camarasaurus, Lourinhasaurus, rebbachisaurids and Euhelopus. In marked contrast, D'Emic (2012, character 25) considered the presence of a thin EPRL passing nearly horizontally across the neural arch to be plesiomorphic for eusauropods, with some form of EPRL present in Shunosaurus, Omeisaurus, Jobaria, Atlasaurus and all macronarians of that study that preserve cervical vertebrae. Reconciling these differences in scoring requires scrutiny of the morphologies that have been interpreted to constitute an EPRL.

In the following sections, we review the muscular and pneumatic soft tissues that sculpt the lateral surface of the neural arch and reinterpret the morphology of several taxa that have been described as having an EPRL, an incipient EPRL, or a stranded epipophyseal lamina. We demonstrate that an anterior ridge of the epipophysis and struts of the SDF can often be recognized as distinct in sauropods and show that the structure called an EPRL in taxa such as Nigersaurus can be rejected as a primary homologue of the EPRL of some non-sauropod sauropodomorphs. In light of our observations, we recommend that anatomical descriptions and phylogenetic characters 
should, where possible, take explicit stock of the distinct morphogenetic effects that different soft tissues can have on vertebral morphology.

Muscular and pneumatic structures of the neural arch. In studies of sauropodomorph vertebral anatomy, the epipophysis is generally considered to comprise the entire extent of any raised, rugose hump above the postzygapophysis. However, research on the osteological correlates of axial musculature in extant birds indicates that the epipophysis - equivalent to the avian torus dorsalis - can be more narrowly described as that raised portion of the dorsal surface of the postzygapophyseal ramus that serves as an attachment site for part of $\mathrm{m}$. longus colli dorsalis and $\mathrm{m}$. ascendens cervicalis (or their non-avian dinosaurian homologues), as well as a few other epaxial muscles (e.g. m. complexus, m. splenius anticus) in the anterior cervical vertebrae (Baumel \& Witmer 1993; Wedel \& Sanders 2002; Tsuihiji 2005). In various extant birds, including Struthio camelus, a distinct ridge or series of ridges is continuous anterolaterally with the region of the postzygapophyseal ramus that serves as an attachment for epipophysis-specific musculature. These ridges mark the attachment of the aponeuroses of another set of epaxial muscles, the mm. intercristales (Fig. 36A; T. Tsuihiji, pers. comm., 2018). In addition to leaving these distinctive scars on the neural arch and anterior surface of the postzygapophysis, the mm. intercristales of Struthio camelus originate on the crista transverso-obliqua (=SPOL) and the lower portion of the neural spine. The crocodilian homologues of these muscles $(\mathrm{mm}$. interarticulares and the lateral, $\mathrm{m}$. tendinoarticularis part of $\mathrm{m}$. transversospinalis cervicis) similarly insert on the anterodorsal aspect of the postzygapophysis (Tsuihiji 2005).

Like Struthio and other extant birds, Klamelisaurus, Euhelopus, Nigersaurus, Sarahsaurus and various other sauropodomorph taxa also exhibit a distinctive, anteriorly directed projection of the epipophysis, which we interpret to be an epaxial muscle scar (Fig. 36). Continuity of this raised ridge with the epipophysis, the distinctly irregular and rugose texture of the ridge (which can be especially striking visa-vis the comparatively smooth, polished surface of the SDF in sauropods), and the expectation from myological studies of extant archosaurs of insertions of epaxial musculature anterolaterally continuous with the epipophysis indicate insertion of $\mathrm{mm}$. intercristales (or its non-avian dinosaurian homologue) along much of this area (Fig. 36). To distinguish separate portions of a contiguous series of muscle attachments in sauropods, we have coined the term 'anterior epipophyseal muscle scar' to refer to the anteriorly positioned attachments of the $\mathrm{mm}$. intercristales, while we use the term 'epipophysis' to refer to the attachment site for $\mathrm{m}$. longus colli dorsalis and $\mathrm{m}$. ascendens cervicalis. The anterior epipophyseal muscle scar is equivalent to what some previous studies have labelled an EPRL (Wilson \& Upchurch 2009, figs 9, 11) or a stranded epipophyseal lamina (Wilson 2012). Continuity of the anterior epipophyseal muscle scar with the epipophysis and the lack of soft tissue preservation in sauropod 
body fossils precludes determination of the precise boundary between muscle attachments for $\mathrm{mm}$. intercristales and epipophysis-specific musculature; in accordance with approximate territories for these muscles or their homologues in extant diapsids, we estimate this boundary to occur near the level of the anterior margin of the postzygapophyseal articular facet (Tsuihiji 2005, fig. 2; Fig. 36A).

Sauropodomorphs also preserve rugose texturing that extends posteriorly from the preepipophysis (when present) along the lateral aspect of the prezygapophysis towards the diapophysis, which likely corresponds to attachments of $\mathrm{m}$. ascendens cervicalis and $\mathrm{mm}$. intertransversarii (or, in the latter case, their non-avian saurian homologues, which comprise portions of the m. longissimus group; Wedel \& Sanders 2002; Tsuihiji 2005, 2007; Fig. 36). In most sauropods (e.g. Cetiosaurus - LEICT G468.1968; Klamelisaurus - IVPP 9497; Camarasaurus - BYU 9047; Diplodocus - CM 84), the lateral surface of the prezygapophyseal ramus exhibits some amount of low, rugose texturing, which does not take on the raised, linear prominence of a lamina. By contrast, in some non-sauropod sauropodomorphs, including Sarahsaurus (TMM 43646-2; TMM 43646-3) and Massospondylus (BP/1/4934), this region forms a laterally raised ridge that extends posteriorly and somewhat ventrally from the prezygapophysis and exhibits subtle rugosities and longitudinal striae (Fig. 36B, C). In anterior and middle cervical vertebrae, this ridge passes ventral to and is distinct from the ridge extending anteriorly from the epipophysis. In more posterior cervical vertebrae, however, these two ridges can merge, forming what was called a 'table-like shelf' by A. D. Marsh \& Rowe (2018) and what Barrett et al. (2019) interpreted as an EPRL. The merging of serially separate ridges to form a single laminar structure bridging the interval between the prezygapophysis and epipophysis indicates that this structure - the EPRL of Barrett et al. (2019) - is serially segmented in these taxa.

In addition to muscle scars, various sauropods preserve conspicuous pneumatic structures in the SDF of their cervical vertebrae. A clearly demarcated SDF is absent in non-sauropod sauropodomorphs (e.g. R. N. Martınez \& Alcober 2009; McPhee \& Choinere 2017; Barrett et al. 2019), but is present as a distinct fossa in Tazoudasaurus and other sauropods (Wilson et al. 2011), where it is understood to house a pneumatic diverticulum of the cervical air sacs (e.g. Janensch 1947; Schwarz \& Fritsch 2006; Schwarz et al. 2007; Wilson et al. 2011). Although many eusauropods have a simple, relatively smooth-walled SDF (e.g. Shunosaurus - Y. Zhang 1988; Cetiosaurus LEICT G468.1968; Jobaria - MNBH TIG 6; Dicraeosaurus - Janensch 1929a; Malawisaurus - Gomani 2005), others develop various pneumatic ridges and rimmed fossae (e.g. Qijianglong - QJGPM 1001; Klamelisaurus - Fig. 5C; Diplodocus Hatcher 1901; Vouivria - Mannion et al. 2017, fig. 6a; Giraffatitan - Janensch 1947). Of particular interest here is the development of sub-horizontal struts of variable prominence, as these laminae, when single and partly or completely crossing the SDF, 
are often called an EPRL (e.g. Haluza et al. 2012; Mocho et al. 2014; Zurriaguz \& Powell 2015; Averianov et al. 2018), or an incipient or stranded variant thereof (e.g. Wilson et al. 2011; Melstrom et al. 2016; Wilson et al. 2019). A smooth-edged lamina extending the full distance between the epipophysis and prezygapophysis is rare among sauropods, although the vertebrae of a few taxa (e.g. cervical 3 of Camarasaurus lewisi and Euhelopus; see below) approximate this condition. More commonly, struts that have been identified as an EPRL in sauropods completely or partially span the interval between the SPRL and PODL, and through these laminae are understood to connect to the epipophysis and prezygapophysis. Under the same reasoning, PRDL-to-SPOL struts are also recognized as an EPRL (Wilson et al. 2011; D'Emic 2012). It is worth noting that on the left side of cervicals 14 and 15 of Klamelisaurus (Figs 5,6) there exists a pair of horizontal struts, neither of which is differentiable on its own from what has been called an incipient EPRL in other taxa.

That the morphologies resulting from the influence of muscle attachments and pneumatic epithelia on the neural arch should, where possible, be considered distinct, rather than broadly homologized as an EPRL, can be demonstrated by an examination of variation in the development of these structures in Camarasaurus, Euhelopus and other sauropods, as we detail below.

Camarasaurus. Serial variation in the morphology of the neural arches of cervicals 3-7 of Camarasaurus lewisi (BYU 9047) provide strong evidence that an anterior epipophyseal muscle scar and a pneumatic strut of the SDF - structures that have been considered on their own to constitute an EPRL or a serial variant thereof (see above) are distinct entities.

In cervical 3 of Camarasaurus lewisi, a nodular, highly rugose ridge tops the posterior end of the postzygapophyseal ramus and anteriorly abuts a stout lamina that crosses the SDF (Fig. 37B, F, J). This latter structure approximates the simplest form of the EPRL described by Wilson et al. (2011), although the anterior, prezygapophyseal end of the lamina intersects the SPRL before reaching the prezygapophysis. Cervical 4 also bears a nodular mass on the dorsal surface of the postzygapophyseal ramus, although the anterior projection of this mass - the anterior epipophyseal muscle scar - extends farther anteriorly towards the SDF than in cervical 3 (Fig. 37C, G, K). The SDF of cervical 4 contains a thin, smooth-edged accessory strut (equivalent to the PODLSPRL lamina of Carballido et al. [2012] and the EPRL of Wilson et al. [2011]) that is essentially confluent with the PODL posteriorly and the SPRL anteriorly. Although the anterior epipophyseal muscle scar approaches the lamina of the SDF, the two structures are easily distinguished in texture and girth, the accessory lamina of the SDF being narrow and having the same, relatively smooth texture as the surrounding SDF. A similar arrangement is present in cervical 5 of Camarasaurus lewisi, although in this 
element, a smooth expanse of the SDF separates the anterior epipophyseal muscle scar from the thin, horizontal accessory lamina of the SDF (Fig. 37D, H, L), as is the case for all preserved cervical elements of Klamelisaurus with accessory lamina(e) (Figs 46). In more posterior elements of Camarasaurus lewisi, the accessory strut of the SDF is absent, but a well-developed anterior epipophyseal muscle scar persists on all preserved cervical vertebrae (Fig. 37E, I, M).

If the anterior epipophyseal muscle scar and the accessory strut of the SDF are interpreted to constitute a single entity, then Camarasaurus lewisi must be considered to have a segmented EPRL in cervical 5 and a stranded epipophyseal lamina in cervical 7 and more posterior vertebrae. We believe it is more precise and anatomically informative to describe the cervical neural arches of Camarasaurus lewisi as bearing two distinct structures, an epaxial muscle scar and a horizontal strut within a presumably pneumatized SDF. This interpretation is supported by the textural differences between the anterior epipophyseal muscle scar and the accessory lamina of the SDF, the serial dissipation of a close correspondence between these structures, and the subsequent disappearance of the horizontal strut but not the anterior epipophyseal muscle scar.

Euhelopus. A similar correspondence between an epipophyseal ridge and a strut of the SDF is apparent in Euhelopus. However, unlike Camarasaurus lewisi, the dissipation of a close association between these structures occurs much farther posteriorly in the cervical series in Euhelopus. The horizontal lamina subdividing the SDF of Euhelopus on the right (but not the left) side of cervical 3 is similar to that described above for cervical 3 of Camarasaurus lewisi and closely resembles the simplest form of the EPRL described by Wilson et al. (2011). The left side of cervical 4 preserves a sharpedged ridge that crosses the SDF and approaches the SPRL, but preservation of the lateral surface of the neural spine is otherwise poor in cervicals 4-6. In cervicals 7-11, a rugose ridge extends anteriorly from the epipophysis into the SDF, towards the prezygapophysis. In most of these vertebrae, this projection tapers dramatically into a thin, low ridge that weakly divides the anterior half of the SDF and dissipates as it approaches the prezygapophysis (Fig. 36E; Wilson \& Upchurch 2009, figs 9, 10). This dramatic distal tapering is reminiscent of the condition present in cervical 4 of Camarasaurus lewisi, in which the anterior epipophyseal muscle scar and horizontal strut of the SDF are in apposition, and might reflect the approximate boundary between the muscular and pneumatic soft tissues of the neural arch. Although the sudden tapering of the ridge in Euhelopus could conceivably reflect a change in the nature of the attachment of mm. intercristales (e.g. to a more aponeurotic attachment), the morphology of subsequent posterior cervical vertebrae leads us to favour the former interpretation. In these elements, a rugose ridge continues to extend anteriorly from the epipophysis into the comparatively smooth-walled SDF, but the thin strut of the SDF 
becomes markedly displaced ventrally from the level of the ridge, producing a stepped configuration (Figs 33A, 36G).

As in Camarasaurus lewisi, if the ridge extending anteriorly from the epipophysis and the thin strut of the SDF are interpreted to constitute a single entity, then Euhelopus must be considered to have a segmented EPRL in posterior cervical vertebrae, where the strut becomes ventrally displaced from the epipophyseal ridge. We prefer to interpret Euhelopus as showing evidence of distinct muscular and pneumatic soft tissues sculpting the lateral surface of the neural arch, producing an epaxial muscle scar and a pneumatic strut of variable prominence whose distinctiveness is most clearly demonstrated in posterior cervical vertebrae. While our interpretation of these structures would indicate that Euhelopus is not autapomorphic in possessing a single, continuous EPRL throughout the cervical series, as proposed by Wilson \& Upchurch (2009), the taxon might remain autapomorphic in the extent of excursion of the anterior epipophyseal muscle scar into the SDF, which is greater than that observed in Klamelisaurus or other taxa with a well-developed muscle scar (e.g. Camarasaurus lewisi; Moabosaurus - Britt et al. 2017, fig. 15).

Nigersaurus. As in Camarasaurus lewisi, Klamelisaurus and Euhelopus, the cervical vertebrae of Nigersaurus have a rugose anterior extension of the epipophysis that can be distinguished from a smooth-edged strut crossing the SDF (MNBH GAD 512). Unlike these taxa, however, in which the muscle scar for the mm. intercristales is a robust projection and the strut of the SDF is generally low and fine, the anterior epipophyseal muscle scar in Nigersaurus is weakly expressed as a thin line of rugosities dorsomedial to the smooth, rounded edge of the PODL, and the strut of the SDF is robust and completely confluent with the PODL posteriorly and the SPRL anteriorly (Fig. 36F). In none of the cervical vertebrae of Nigersaurus MNBH GAD 512 that we examined (cervicals 3 and 5-10) does the strut of the SDF conform to the simplest example of an EPRL described by Wilson et al. (2011); instead, it lacks direct continuity with either the epipophysis or prezygapophysis, and manifests in cervicals 5-10 as a stout lateral strut that merges with the SPRL and PODL. In all of these vertebrae, the anterior epipophyseal muscle scar dissipates before reaching the posterior end of the PODL-SPRL strut (Fig. 36F). Wilson (2012, fig. 3) labelled both the anteriorly projecting rugosities of the epipophysis and the bracing strut of the SDF as an EPRL. We prefer to describe these features in Nigersaurus as distinct, one arising from epaxial muscle attachments and the other in contact with the pneumatic epithelium of the SDF.

Uberabatitan. Recognizing that epaxial musculature can produce anterior projections of the epipophysis offers an alternative explanation for the development of 'segmented laminae' in some titanosaurians (Salgado \& de Souza Carvalho 2008; Wilson 2012; 
Silva Junior et al. 2019). In diagnosing the Brazilian titanosaurian Uberabatitan, Salgado \& de Souza Carvalho (2008) described the PODL of anterior and middle cervical vertebrae as being segmented into two unconnected laminae, with a zygapophyseal segment of the PODL ('PODL(z)') extending anterodorsally over a diapophyseal segment ('PODL(d)') (Fig. 36H). As discussed above, Wilson (2012) instead interpreted these structures as stranded serial variants of the EPRL and PODL. From the published images of these specimens, the dorsal of the two structures appears to be rugose and stouter where it overlaps the ventral lamina (Salgado \& de Souza Carvalho 2008, figs 4-6; Wilson 2012, fig. 8; Silva Junior et al. 2019, figs 3, 4). As discussed by Wilson (2012), these two structures can be very similar in orientation, and - if acting as biomechanical braces - would seem to be semi-redundant in resisting similar forces.

If the stranded epipophyseal lamina, or PODL(z), is instead reinterpreted as an epaxial muscle scar - the anterior epipophyseal muscle scar of this study - this confusion dissolves: the ridge need no longer be explained within a paradigm that anticipates elongate ridges of the neural spine to be bracing struts that resist compression or separate pneumatic fossae, and there is no reason to expect an epaxial muscle scar to link separate landmarks of a single vertebra. Notably, the conformation of the anterior epipophyseal muscle scar and the PODL in Uberabatitan is strikingly similar to that observed in Klamelisaurus and Euhelopus (Fig. 36D, E, G, H): the muscle scar overlaps and passes the PODL anterodorsally, and, as in some cervical vertebrae of Klamelisaurus (Fig. 36D), the PODL dissipates before fully reaching the postzygapophysis. Although our explanation resolves the seemingly anomalous development of segmented or semi-redundant laminae in sauropod cervical vertebrae, it leaves open the question of the functional significance of serial and interspecific variation in the robusticity and anterior extent of the anterior epipophyseal muscle scar. Addressing such questions is an area for future research.

Primary homology of ridges of the neural arch in sauropodomorphs. Having reviewed and reinterpreted the identity of ridges of the neural arch in various sauropods, we now consider how different expert observers could recognize an EPRL in eusauropods as either ubiquitous and symplesiomorphic (D'Emic 2012, character 25) or rare and homoplastic (Mannion et al. 2019, character 129). This difference of opinion appears to turn, at least in part, on whether 'incipient' or variable struts of the SDF are considered an EPRL. Recognition of an EPRL by Mannion et al. (2019) is limited to those taxa with a robust horizontal strut that fully (or nearly) spans the SDF, as exemplified by Nigersaurus, Limaysaurus (Calvo \& Salgado 1995) and the posterior-most cervical vertebrae of Euhelopus (Fig. 33A). By contrast, D’Emic (2012) scored an EPRL as present in taxa without robust horizontal struts (Jobariaarticulated cervicals 2-10 [MNBH TIG 6] lack struts in their SDF, but a cervicodorsal 
vertebra labelled 'F257' has a low, short strut in its left SDF) or that have variable pneumatic structures, but no singularly distinct horizontal strut (Europasaurus Carballido \& Sander 2014; Giraffatitan - Janensch 1950, MB.R.2180, MB.R. 2181). Although these taxa appear to lack singular, clearly demarcated, horizontal struts of the SDF, they have well-developed epipophyseal rugosities that extend up to or slightly beyond the anterior margin of the postzygapophyseal facet, which could be reasonably considered an EPRL (or stranded epipophyseal lamina) following previous work (Wilson \& Upchurch 2009; Wilson et al. 2011; Wilson 2012). Whatever the cause of these differences in scoring, that they exist for a structure that, as a named vertebral lamina, is meant to be objectively and repeatably identifiable across researchers, speaks to the lack of clarity surrounding the EPRL.

Reconciling differences in interpretation will be particularly important as phylogenetic matrices focusing on eusauropods are expanded to include non-sauropod sauropodomorphs. As a case in point, Barrett et al. (2019) described an EPRL in the posterior cervical vertebrae of Massospondylus and a similar feature is present in Sarahsaurus (TMM 43646-2, 43646-3). As noted above, this structure forms serially from the union of an anteriorly projecting ridge of the epipophysis and a posteriorly projecting ridge that originates on the prezygapophysis. Both of these ridges bear fine rugosities and longitudinal striae that indicate they were attachment sites for cervical muscles (Fig. 36B, C). Whereas these two bodies of muscle scars are in close proximity in Sarahsaurus and Massospondylus, the homologous rugosities in sauropods are well removed from one another, and are separated by the SDF (Fig. 36). No cervical vertebra of Massospondylus or Sarahsaurus has an SDF or shows evidence of external pneumatization. Thus, the ridge that connects the epipophysis to the prezygapophysis in the posterior cervical vertebrae of these taxa cannot be considered a primary homologue of the smooth-edged struts situated in the pneumatized SDF of taxa such as Nigersaurus and Klamelisaurus. The important point here is that connectivity and position alone are an insufficient basis for rejecting the homology of the 'EPRL' of Massospondylus with the 'EPRL' of Nigersaurus. As we have sought to demonstrate throughout this discussion, recourse to the inferred influence of morphogenetic soft tissues is required to make defensible hypotheses of primary homology for some structures that have previously been considered an EPRL.

To be clear, we are not arguing that muscles always leave discrete attachment scars, or that every stranded ridge near the epipophysis was produced by a muscle. Cervical 3 of Jobaria (MNBH TIG 6), for example, exhibits a stranded lamina without rugose texturing that projects anteroventrally from the postzygapophysis, which is most likely a serial variant of the PODL. Although differences in the texture and position of muscular and pneumatic structures are abrupt and distinct in the taxa discussed above, we recognize that this is not always the case (Apostolaki et al. 2015); in such instances, 
it may not be possible to make primary homology statements like those advanced here. We also are not arguing that the axial musculature and vertebral laminae of sauropodomorphs existed in isolation from one another: laminae often bear rugose texturing that suggests muscle or ligament attachments, and the autapomorphic bony projections of the cervical SPRLs in Klamelisaurus were presumably produced by the attachment of epaxial musculature.

Instead, we have tried to demonstrate that, in sauropodomorphs, some previous identifications of the EPRL for the purposes of descriptive anatomy and phylogenetic character construction have almost certainly conflated distinct muscular and pneumatic structures as components of a single entity. We argue that the osteological products of these soft tissues can often be distinguished, and that when they can, it is more anatomically informative and precise to describe these structures separately for the reason that they show a capacity for independent development in sauropods. Accordingly, morphological characters should be constructed so as to exploit the independent phylogenetic information that these structures carry; this is the approach we have taken in the phylogenetic analyses presented here, with separate characters for the presence of an anterior epipophyseal muscle scar (GEA character 435; CEA character 386) and singular bridging struts of the SDF (GEA character 129; CEA character 119), both of which avoid the term 'EPRL' in favour of direct descriptions of morphology. Given the substantial variability in shape and relative size of the anterior epipophyseal muscle scar and pneumatic struts of the SDF across sauropodomorphs, we anticipate that additional phylogenetic information remains to be gleaned from careful comparative study of these structures.

\section{Conclusions}

Our detailed description and reassessment of Klamelisaurus indicates that it is a valid taxon that can be distinguished from other sauropods from the Middle-Late Jurassic of China. Comparisons to the Early Cretaceous taxon Euhelopus reveal several striking similarities. Applying parsimony and time-calibrated Bayesian phylogenetic inference to two character matrices incorporating these new observations and numerous additional East Asian eusauropods places Klamelisaurus in a non-macronarian Euhelopodidae in most of our analyses, calling into question the macronarian affinities of Euhelopus and indicating that rigorous testing of the phylogenetic position of the taxon in future analyses requires inclusion of a diversity of Middle-Late Jurassic East Asian forms, including Klamelisaurus. The phylogenetic and comparative anatomical data presented here provide a foundation for future revision of the taxonomy and systematics of sauropods from the Junggar and Sichuan basins, with re-description of the type species of Mamenchisaurus and Omeisaurus being obvious first steps. The difficulty of clearly recognizing in the cervical vertebrae of Klamelisaurus whether an 
anterior epipophyseal muscle scar or horizontal strut of the SDF (or both, or neither) are appropriately considered a primary homologue of the EPRL is symptomatic of a wider lack of clarity surrounding repeatable recognition of this structure. Anatomical comparisons of the cervical vertebrae of Klamelisaurus to several other sauropodomorphs and insights from myological studies of extant archosaurs strongly suggest that osteological correlates of muscular and pneumatic soft tissues - sometimes conflated as a single EPRL - produce a diversity of morphologies on the lateral surface of the neural spine and postzygapophyseal ramus of sauropod cervical vertebrae, the functional significance of which is a potentially rich area of future inquiry.

\section{Acknowledgements}

This paper represents a portion of the doctoral thesis of AJM conducted at The George Washington University. For hospitality and access to specimens in their care, we wish to thank A. Henrici (CM), B. Britt \& R. Scheetz (BYU), R. Royo-Torres (CPT), J. Choiniere (ESI), Zheng F. \& Geng BH. (IVPP), M. Evans (LEICT), M. Belinchon (MCNV), S. Chapman (NHMUK), M. Herrero (MPG \& ZH), D. Schwarz-Wings \& T. Schossleitner (MB.R.), H. Ketchum (OUMNH), P. Sereno \& T. Keillor (University of Chicago), M. Brown \& C. Sagebiel (TMM), and Jiang S., Peng GZ, Li F. \& Ouyang H. (ZDM). Fieldwork on the Navajo Nation Reservation that led to the excavation of Sarahsaurus aurifontanalis was conducted under a permit from the Navajo Nation Minerals Department. Any persons wishing to conduct geological investigations on the Navajo Nation Reservation must first apply for and receive a permit from the Navajo Nation Minerals Department, P. O. Box 1910, Window Rock, Arizona 86515, telephone number (928) 871-6587. We are grateful to S. Poropat and B. Kear for scans of Euhelopus cranial material (PMU 24705). J. Wilson, S. Poropat, B. Kear, T. Sekiya, T. Miyashita and J. Carballido all kindly provided photographs of taxa used in our phylogenetic analyses. We thank Feng Y. and Hou Y.-M. for assistance with CT scanning. The authors are indebted to Wang S., D. Hone, C. Sullivan, Zhou Z., Wang Y., Qin Z.-C., Zhou Y., Wang J., Liao J.-Q. and Li F. for their unflagging generosity during research trips to Beijing. We gratefully acknowledge the Willi Hennig Society for sponsoring the development and free distribution of TNT. Translations of Dong et al. (1983), Zhao (1993), He et al. (1996), Pi et al. (1996), Y. Zhang et al. (1998) and Fang et al. (2000) were carried out by Will Downs and the translation of Janensch (1947) was carried out by G. Maier; these translations were obtained courtesy of the Polyglot Paleontologist website (http://www.paleoglot.org). Wang S. kindly assisted with translations of Zhou et al. (2018). E. Schweizerbart'sche Verlagsbuchhandlung (http://www.schweizerbart.de/) kindly provided permission to reproduce an image from Janensch (1961) in Figure 34. Thoughtful reviews by J. Wilson, an anonymous reviewer, and the Associate Editor significantly improved the manuscript. AJM was 
supported by the National Science Foundation (DGE-1246908; OISE 1515288), Jurassic Foundation, Cosmos Club and George Washington University, and wishes to thank C. Forster, J. Stiegler, A. Pyron and J. Wilson for useful discussion. This work was also supported by grants from The Royal Society of London to PU and PMB; from the Leverhulme Trust to PU; from the Earth Sciences Departmental Investment Fund (Natural History Museum) to PMB; from the National Geographic Society and the National Science Foundation (EAR 0922187 and EAR 0310217) to JMC; and from the National Natural Science Foundation of China (No. 41688103, 91514302, 41120124002) and the Strategic Priority Research Program of the Chinese Academy of Sciences (Grant No. XDB18030504) to XX.

\section{ORCID}

Andrew J. Moore @ittp://orcid.org/0000-0002-3023-2502

\section{References}

Alifanov, V. R. \& Averianov, A. O. 2003. Ferganasaurus verzilini, gen. et sp. nov., a new neosauropod (Dinosauria, Saurischia, Sauropoda) from the Middle Jurassic of Fergana Valley, Kirghizia. Journal of Vertebrate Paleontology, 23, 358-372. doi:10.1671/02724634(2003)023[0358:FVGESN]2.0.CO;22.0.CO;2]

Allain, R. \& Aquesbi, N. 2008. Anatomy and phylogenetic relationships of Tazoudasaurus naimi (Dinosauria, Sauropoda) from the late Early Jurassic of Morocco. Geodiversitas, 30, 345-424.

Altekar, G., Dwarkadas, S., Huelsenbeck, J. P. \& Ronquist, F. 2004 . Parallel Metropolis coupled Markov chain Monte Carlo for Bayesian phylogenetic inference. Bioinformatics, 20, 407-415. doi:10.1093/bioinformatics/btg427

Apostolaki, N. E., Rayfield, E. J. \& Barrett, P. M. 2015. Osteological and soft-tissue evidence for pneumatization in the cervical column of the ostrich (Struthio camelus) and observations on the vertebral columns of non-volant, semi-volant and semi-aquatic birds. PLoS ONE, 10, e0143834. doi:10.1371/journal.pone.0143834

Averianov, A., Ivantsov, S., Skutschas, P., Faingertz, A. \& Leshchinskiy, S. 2018. A new sauropod dinosaur from the Lower Cretaceous Ilek Formation, Western Siberia, Russia. Geobios, 51, 1-14. doi:10.1016/j.geobios.2017.12.004 
Barrett, P. M. \& Wang, X.-L. 2007. Basal titanosauriform (Dinosauria, Sauropoda) teeth from the Lower Cretaceous Yixian Formation of Liaoning Province, China. Palaeoworld, 16, 265-271. doi:10.1016/j.palwor.2007.07.001

Barrett, P. M., McGowan, A. J. \& Page, V. 2009. Dinosaur diversity and the rock record. Proceedings of the Royal Society B, 276, 2667-2674. doi:10.1098/rspb.2009.0352

Barrett, P. M., Hasegawa, Y., Manabe, M., Isaji, S. \& Matsuoka, H. 2002. Sauropod dinosaurs from the Lower Cretaceous of eastern Asia: taxonomic and biogeographical implications. Palaeontology, 45, 1197-1217. doi:10.1111/1475-4983.00282

Barrett, P. M., Chapelle, K. E. J., Staunton, C. K., Botha, J. \& Choiniere, J. N. 2019. Postcranial osteology of the neotype specimen of Massospondylus carinatus Owen, 1854 (Dinosauria: Sauropodomorpha) from the upper Elliot formation of South Africa. Palaeontologia Africana, 53, 114-178.

Baumel, J. J. \& Witmer, L. M. 1993. Osteologia. Pp. 45-132 in J. J. Baumel, A. S. King, J. E. Breazile, H. E. Evans \& J. C. Vanden Berge (eds) Handbook of avian anatomy: nomina anatomica avium. Publications of the Nuttall Ornithological Club, Cambridge, USA.

Bever, G. S., Gauthier, J. A. \& Wagner, G. P. 2011. Finding the frame shift: digit loss, developmental variability, and the origin of the avian hand. Evolution \& Development, 13, 269279. doi:10.1111/j.1525-142X.2011.00478.X

Bonaparte, J. F. 1986a. Les dinosaures (Carnosaures, Allosaurides, Sauropodes, Cetiosaurides) du Jurassique Moyen de Cerro Condor (Chubut, Argentine). Annales de Paleontologie, 72, 325386.

Bonaparte, J. F. 1986b. The early radiation and phylogenetic relationships of the Jurassic sauropod dinosaurs, based on vertebral anatomy. Pp. 247-258 in K. Padian (ed.) The beginning of the age of dinosaurs. Cambridge University Press, Cambridge.

Bonaparte, J. F. 1996. Cretaceous tetrapods of Argentina. Pp. 73-130 in G. Arratia (ed.) Contributions of southern South America to vertebrate paleontology. M€unchner Geowissenschaftliche Abhandlungen, Reihe A, Geologie und Pal€aontologie, 30.

Bonaparte, J. F., Heinrich, W.-D. \& Wild, R. 2000. Review of Janenschia Wild, with the description of a new sauropod from the Tendaguru beds of Tanzania and a discussion on the systematic value of procoleous caudal vertebrae in the Sauropoda. Palaeontographica, Abteilung A, 256, 25-76.

Bonnan, M. F. 2005. Pes anatomy in sauropod dinosaurs: implications for functional morphology, evolution, and phylogeny. Pp. 346-380 in V. Tidwell \& K. Carpenter (eds) Thunder-lizards: the sauropodomorph dinosaurs. Indiana University Press, Bloomington and Indianapolis. 
Borsuk-Białynicka, M. 1977. A new camarasaurid sauropod Opisthocoelicaudia skarzynskii, gen. n., sp. n. from the Upper Cretaceous of Mongolia. Palaeontologia Polonica, 37, 1-64.

Bouckaert, R., Heled, J., Ku€hnert, D., Vaughan, T., Wu, C-H., Xie, D., Suchard, M. A., Rambaut, A. \& Drummond, A. J. 2014. BEAST 2: a software platform for Bayesian evolutionary analysis. PLoS Computational Biology, 10(4), e1003537. doi:10.1371/journal.pcbi. 1003537

Britt, B. B., Scheetz, R. D., Whiting, M. F. \& Wilhite, D. R. 2017. Moabosaurus utahensis, n. gen., n. sp., a new sauropod from the Early Cretaceous (Aptian) of North America. Contributions from the Museum of Paleontology, University of Michigan, 32, 189-243.

Bussert, R., Heinrich, W.-D. \& Aberhan, M. 2009. The Tendaguru Formation (Late Jurassic to Early Cretaceous, southern Tanzania): definition, palaeoenvironments, and sequence stratigraphy. Fossil Record, 12, 141-174, doi:10. 1002/mmng.200900004.

Buffetaut, E., Suteethorn, V., Cuny, G., Tong, H., Loeuff, J. L., Khansubha, S. \& Jongautchariyakul, S. 2000. The earliest known sauropod dinosaur. Nature, 407, 72-74. doi: $10.1038 / 35024060$

Calvo, J. O. \& Salgado, L. 1995. Rebbachisaurus tessonei sp. nov. a new Sauropoda from the Albian-Cenomanian of Argentina; new evidence on the origin of the Diplodocidae. Gaia, 11, 1333.

Campione, N. E., Brink, K. S., Freedman, E. A., McGarrity, C. T. \& Evans, D. C. 2013. 'Glishades ericksoni', an indeterminate juvenile hadrosaurid from the Two Medicine Formation of Montana: implications for hadrosauroid diversity in the latest Cretaceous (CampanianMaastrichtian) of western North America. Palaeobiodiversity and Palaeoenvironments, 93, 6575. doi:10.1007/s12549-012-0097-1

Canudo, J. I., Ruiz-Omen aca, J. I., Barco, J. L. \& Royo Torres, R. 2002. >Sauropodos asiaticos en el Barremiense inferior (Cretacico Inferior) de Espa na? Ameghiniana, 39, 443-452.

Carballido, J. L., Rauhut, O. W. M., Pol, D. \& Salgado, L. 2011. Osteology and phylogenetic relationships of Tehuelchesaurus benitezii (Dinosauria, Sauropoda) from the Upper Jurassic of Patagonia. Zoological Journal of the Linnean Society, 163, 605-662. doi:10.1111/j.1096-3642. 2011.00723.x

Carballido, J. L., Marpmann, J. S., Schwarz-Wings, D. \& Pabst, B. 2012. New information on a juvenile sauropod specimen from the Morrison Formation and the reassessment of its systematic position. Palaeontology, 55, 567-582. doi:10.1111/j.1475-4983.2012.01139.x

Carballido, J. L. \& Sander, P. M. 2014. Postcranial axial skeleton of Europasaurus holgeri (Dinosauria, Sauropoda) from the Upper Jurassic of Germany: implications for sauropod ontogeny and phylogenetic relationships of basal Macronaria. Journal of Systematic Palaeontology, 12, 335-387. doi:10.1080/14772019.2013.764935 
Carballido, J. L., Pol, D., Ruge, M. L. P., Bernal, S. P., Paramo-Fonseca, M. E. \& Etayo-Serna, F. 2015. A new Early Cretaceous brachiosaurid (Dinosauria, Neosauropoda) from northwestern Gondwana (Villa de Leiva, Colombia). Journal of Vertebrate Paleontology, 35, e980505. doi: 10.1080/02724634.2015.980505

Carballido, J. L., Pol, D., Otero, A., Cerda, I. A., Salgado, L., Garrido, A. C., Ramezani, J., Cuneo, N. R. \& Krause, J. M. 2017. A new giant titanosaur sheds light on body mass evolution among sauropod dinosaurs. Proceedings of the Royal Society B, 284, 20171219. doi: 10.1098/ rspb.2017.1219

Carpenter, K. \& McIntosh, J. S. 1994. Upper Jurassic sauropod babies from the Morrison Formation. Pp. 265-278 in K. Carpenter, K. F. Hirsch \& J. R. Horner (eds) Dinosaur eggs and babies. Cambridge University Press, Cambridge.

Cerda, I. A., Casal, G. A., Martinez, R. D. \& Ibiricu, L. M. 2015. Histological evidence for a supraspinous ligament in sauropod dinosaurs. Royal Society Open Science, 2, 150369. doi: 10.1098/rsos.150369

Choiniere, J. N., Clark, J. M., Forster, C. A., Norell, M. A., Eberth, D. A., Erickson, G. M., Chu, H. \& Xu, X. 2013. A juvenile specimen of a new coelurosaur (Dinosauria: Theropoda) from the Middle-Late Jurassic Shishugou Formation of Xinjiang, People's Republic of China. Journal of Systematic Palaeontology, 12, 177-215. doi:10.1080/14772019.2013.781067

Clark, J. M., Xu, X., Eberth, D. A., Forster, C. A., Malkus, M., Hemming, S. \& Hernandez, R. 2006. The Middle to Late Jurassic terrestrial transition: new discoveries from the Shishugou Formation, Xinjiang, China. Pp. 26-28 in P. M. Barrett \& S. E. Evans (eds) Ninth international symposium on Mesozoic terrestrial ecosystems and biota. Natural History Museum, London.

Cooper, M. R. 1984. A reassessment of Vulcanodon karibaensis Raath (Dinosauria: Saurischia) and the origin of the Sauropoda. Palaeontologia Africana, 25, 203-231.

Currie, P. J., Holmes, R. B., Ryan, M. J. \& Coy, C. 2016. A juvenile chasmosaurine ceratopsid (Dinosauria, Ornithischia) from the Dinosaur Park Formation, Alberta, Canada. Journal of Vertebrate Paleontology, 36, e1048348. doi:10.1080/02724634.2015.1048348

Curry Rogers, K. A. 2005. Titanosauria: a phylogenetic overview. Pp. 50-103 in K. A. Curry Rogers \& J. A. Wilson (eds) The sauropods: evolution and paleobiology. University of California Press, Berkeley.

D'Emic, M. D. 2012. The early evolution of titanosauriform sauropod dinosaurs. Zoological Journal of the Linnean Society, 166, 624-671. doi:10.1111/j.1096-3642.2012.00853.x

D'Emic, M. D. 2013. Revision of the sauropod dinosaurs of the Lower Cretaceous Trinity Group, southern USA, with the description of a new genus. Journal of Systematic Palaeontology, 11, 707-726. doi:10.1080/14772019.2012. 667446 
D'Emic, M. D., Wilson, J. A. \& Williamson, T. E. 2011. A sauropod dinosaur pes from the latest Cretaceous of North America and the validity of Alamosaurus sanjuanensis (Sauropoda, Titanosauria). Journal of Vertebrate Paleontology, 31, 1072-1079. doi:10.1080/02724634.2011. 595856

D'Emic, M. D. \& Foreman, B. Z. 2012. The beginning of the sauropod dinosaur hiatus in North America: insights from the Lower Cretaceous Cloverly Formation of Wyoming. Journal of Vertebrate Paleontology, 32, 883-902. doi:10.1080/02724634.2012.671204

D’Emic, M. D., Foreman, B. Z. \& Jud, N. A. 2016. Anatomy, systematics, paleoenvironment, growth, and age of the sauropod dinosaur Sonorasaurus thompsoni from the Cretaceous of Arizona, USA. Journal of Paleontology, 90, 102-132. doi:10.1017/jpa.2015.67

Dong, Z. 1990. Sauropoda from the Kelameili Region of the Junggar Basin, Xinjiang Autonomous Region. Vertebrata PalAsiatica, 28, 43-58.

Dong, Z. 1997. A gigantic sauropod (Hudiesaurus sinojapanorum gen. et sp. nov.) from the Turpan Basin, China. Pp. 102-110 in Z. Dong (ed.) Sino-Japanese Silk Road dinosaur expedition. China Ocean Press, Beijing.

Dong, Z., Zhou, S. \& Zhang, Y. 1983. Dinosaurs from the Jurassic of Sichuan. Palaeontologica Sinica, Series C, 162, 1-136.

Dong, Z. \& Tang, Z. 1984. Note on a new mid-Jurassic sauropod (Datousaurus bashanensis gen. et sp. nov.) from Sichuan Basin, China. Vertebrata PalAsiatica, 22, 69-75.

Fang, X., Long, Q., Lu, L., Zhang, Z., Pan, S., Wang, Y., Li, X. \& Cheng, Z. 2000. Lower, Middle, and Upper Jurassic subdivision in the Lufeng region, Yunnan Province. Pp. 208-214 in Editorial Committee (ed.) Proceedings of the third national stratigraphical congress of China. Geological Publishing House, Beijing.

Farke, A. A. \& Sertich, J. J. W. 2013. An abelisauroid theropod dinosaur from the Turonian of Madagascar. PLoS ONE, 8, e62047, doi: 10.1371/journal.pone.0062047 Fernandez-Baldor, F. T., Canudo, J. I.,

Huerta, P., Moreno-Azanza, M. \& Montero, D. 2017. Europatitan eastwoodi, a new sauropod from the Lower Cretaceous of Iberia in the initial radiation of somphospondylans in Laurasia. PeerJ, 5, e3409. doi:10.7717/peerj.3409

Gavryushkina, A., Welch, D., Stadler, T. \& Drummond, A. 2014. Bayesian inference of sampled ancestor trees for epidemiology and fossil calibration. PLoS Computational Biology, 10, e1003919. doi:10.1371/journal.pcbi.1003919

Gilmore, C. W. 1936. Osteology of Apatosaurus: with special reference to specimens in the Carnegie Museum. Memoirs of the Carnegie Museum, 11, 175-300. 
Goloboff, P. A., Farris, J. S., K€allersj€o, M., Oxelman, B., Ramıacuterez, M. J. \& Szumik, C. A. 2003. Improvements to resampling measures of group support. Cladistics, 19, 324-332. doi: 10.1111/j.1096-0031.2003. tb00376.x

Goloboff, P., Farris, J. S. \& Nixon, K. C. 2008. TNT, a free program for phylogenetic analysis. Cladistics, 24, 774-786. doi:10.1111/j.1096-0031.2008.00217.x

Goloboff, P. A. 2014. Extended implied weighting. Cladistics, 30, 260-272. doi:10.1111/cla. 12047

Goloboff, P. A. \& Catalano, S. A. 2016. TNT version 1.5, including a full implementation of phylogenetic morphometrics. Cladistics, 32, 221-238. doi:10.1111/cla. 12160

Goloboff, P. A., Torres, A. \& Arias, J. S. 2017. Weighted parsimony outperforms other methods of phylogenetic inference under models appropriate for morphology. Cladistics, 34, 407-437. doi:10.1111/cla.12205

Goloboff, P. A., Pittman, M., Pol, D. \& Xu, X. 2019. Morphological data sets fit a common mechanism much more poorly than DNA sequences and call into question the Mkv Model. Systematic Biology, 68, 494-504. doi:10. 1093/sysbio/syy077

Gomani, E. M. 2005. Sauropod dinosaurs from the Early Cretaceous of Malawi, Africa. Palaeontologia Electronica, 8, 1-37.

Gonzalez Riga, B. J., Previtera, E. \& Pirrone, C. A. 2009. Malarguesaurus florenciae gen. et sp. nov., a new titanosauriform (Dinosauria, Sauropoda) from the Upper Cretaceous of Mendoza, Argentina. Cretaceous Research, 30, 135-148. doi:10.1016/j.cretres.2008.06.006

Gonzalez Riga, B. J., Lamanna, M. C., David, L. D. O., Calvo, J. O. \& Coria, J. P. 2016. A gigantic new dinosaur from Argentina and the evolution of the sauropod hind foot. Scientific Reports, 6, 19165. doi:10.1038/ srep19165

Gonzalez Riga, B. J., Mannion, P. D., Poropat, S. F., David, O., D, L. \& Coria, J. P. 2018. Osteology of the Late Cretaceous Argentinean sauropod dinosaur Mendozasaurus neguyelap: implications for basal titanosaur relationships. Zoological Journal of the Linnean Society, 184, 136-181. doi:10.1093/zoolinne/zlx103

Gorscak, E. \& O’Connor, P. M. 2016. Time-calibrated models support congruency between Cretaceous continental rifting and titanosaurian evolutionary history. Biology Letters, 12, 20151047. doi:10.1098/rsbl.2015.1047

Haluza, A., Canale, J. I., Otero, A., Perez, L. M. \& Scanferla, C. A. 2012. Changes in vertebral laminae across the cervicodorsal transition of a well-preserved rebbachisaurid (Dinosauria, Sauropoda) from the Cenomanian of Patagonia, Argentina. Journal of Vertebrate Paleontology, 32, 219-224. doi:10.1080/02724634.2012.620674 
Han, F.-L., Forster, C. A., Clark, J. M. \& Xu, X. 2015. Cranial anatomy of Yinlong downsi (Ornithischia: Ceratopsia) from the Upper Jurassic Shishugou Formation of Xinjiang, China. Journal of Vertebrate Paleontology, 36, e1029579. doi:10.1080/02724634.2015.1029579

Harris, J. D. 2006. The significance of Suuwassea emilieae (Dinosauria: Sauropoda) for flagellicaudatan intrarelationships and evolution. Journal of Systematic Palaeontology, 4, 185198. doi:10.1017/ S1477201906001805

Hatcher, J. 1901. Diplodocus (Marsh): its osteology, taxonomy, and probable habits, with a restoration of the skeleton. Memoirs of the Carnegie Museum, 1, 1-63.

Hatcher, J. 1903. Osteology of Haplocanthosaurus, with description of a new species and remarks on the probable habits of the Sauropoda and the age and origin of the Atlantosaurus beds. Memoirs of the Carnegie Museum, 2, 1-72.

He, X., Li, K. \& Cai, K. 1988. The Middle Jurassic dinosaur fauna from Dashanpu, Zigong, Sichuan. IV. Sauropod dinosaurs (2). Omeisaurus tianfuensis. Sichuan Publishing House of Science and Technology, Chengdu, 143 pp.

He, X., Yang, S., Cai, K., Li, K. \& Liu, Z. 1996. A new species of sauropod, Mamenchisaurus anyuensis sp. nov. Pp. 83-86 in Anonymous (ed.) Papers on geosciences contributed to the 30th International Geological Congress. Chengdu University of Technology, Chengdu.

Horner, J. R., Woodward, H. N. \& Bailleul, A. M. 2016. Mineralized tissues in dinosaurs interpreted as having formed through metaplasia: a preliminary evaluation. Comptes Rendus Palevol, 15, 176-196. doi:10.1016/j.crpv. 2015.01.006

Huene, F. von. 1932. Die fossil Reptil-Ordnung Saurischia, ihre Entwicklung und Geschichte. Monographien zur Geologie und Palaeontologie, 1, 1-361.

Ibiricu, L. M., Casal, G. A., Martinez, R. D., Lamanna, M. C., Luna, M. \& Salgado, L. 2013. Katepensaurus goicoecheai, gen. et sp. nov., a Late Cretaceous rebbachisaurid (Sauropoda, Diplodocoidea) from central Patagonia, Argentina. Journal of Vertebrate Paleontology, 33, 13511366. doi:10.1080/02724634.2013.776562

Ibiricu, L. M., Casal, G. A., Martınez, R. D., Lamanna, M. C., Luna, M. \& Salgado, L. 2015. New material of Katepensaurus goicoecheai (Sauropoda: Diplodocoidea) and its significance for the morphology and evolution of Rebbachisauridae. Ameghiniana, 52, 430-446. doi:10. 5710/ AMGH.24.04.2015.2830

Ibiricu, L., Lamanna, M., Martinez, R., Casal, G., Cerda, I., Martinez, G. \& Salgado, L. 2017. A novel form of postcranial skeletal pneumaticity in a sauropod dinosaur: implications for the paleobiology of Rebbachisauridae. Acta Palaeontologica Polonica, 62, 221-236. doi:10.4202/ app.00316.2016

Ikejiri, T. 2005. Distribution and biochronology of Camarasaurus (Dinosauria, Sauropoda) from the Jurassic Morrison Formation of the Rocky Mountain Region. Pp. 367-379 in S. G. Lucas, Z. 
E. Zeigler, V. W. Lueth \& D. E. Owen (eds) Geology of the Chama Basin, guidebook, 56th field conference. New Mexico Geological Society, Socorro.

Janensch, W. 1929a. Die Wirbels€aule der Gattung Dicraeosaurus. Palaeontographica (Supplement 7), 2, 38-133.

Janensch, W. 1929b. Material und Formegehalt der Sauropoden in der Ausbeute der TendaguruExpedition, 1909-1912. Palaeontographica (Supplement 7), 2, 3-34.

Janensch, W. 1947. Pneumatizitat bei Wirbeln von Sauropoden und anderen Saurischien. Palaeontographica, (Supplement 7), 3, 1-25.

Janensch, W. 1950. Die Wirbelsäule von Brachiosaurus brancai. Palaeontographica (Supplement 7), 7, 27-93. Janensch, W. 1961. Die Gleidmaszen und Gliedmaszengürtel der Sauropoden der Tendaguru-Schichten. Palaeontographica (Supplement 7), 3, 177-235.

Jensen, J. A. 1988. A fourth new sauropod dinosaur from the Upper Jurassic of the Colorado Plateau and sauropod bipedalism. The Great Basin Naturalist, 48, 121-145. Jiang, S., Li, F., Peng, G.-Z. \& Ye, Y. 2011. A new species of Omeisaurus from the Middle Jurassic of Zigong,

Sichuan. Vertebrata PalAsiatica, 49, 185-194.

Kammerer, C. F. 2011. Systematics of the Anteosauria (Therapsida: Dinocephalia). Journal of Systematic Palaeontology, 9, 261-304. doi:10.1080/14772019.2010. 492645

Kass, R. E. \& Raftery, A. E. 1995. Bayes factors. Journal of the American Statistical Association, 90, 773-795. doi:10. 1080/01621459.1995.10476572

Ksepka, D. T. \& Norell, M. 2006. Erketu ellisoni, a long- necked sauropod from Bor Guve (Dornogov Aimag, Mongolia). American Museum Novitates, 3508, 1-16.

Ksepka, D. T. \& Norell, M. A. 2010. The illusory evidence for Asian Brachiosauridae: new material of Erketu ellisoni and a phylogenetic reappraisal of basal Titanosauriformes. American Museum Novitates, 3700, 1-27. doi:10.1206/3700.2

Lacovara, K. J., Lamanna, M. C., Ibiricu, L. M., Poole, J. C., Schroeter, E. R., Ullmann, P. V., Voegele, K. K., Boles, Z. M., Carter, A. M., Fowler, E. K., Egerton, V. M., Moyer, A. E., Coughenour, C. L., Schein, J. P., Harris, J. D., Martınez, R. D. \& Novas, F. E. 2014. A gigantic, exceptionally complete titanosaurian sauropod dinosaur from southern Patagonia, Argentina. Scientific Reports, 4, 6196. doi:10.1038/srep06196

Langer, M. C. 2014. The origins of Dinosauria: much ado about nothing. Palaeontology, 57, 469-478. doi:10.1111/ pala.12108

Lemoine, F., Entfellner, J.-B. D., Wilkinson, E., Correia, D., Felipe, M. D., Oliveira, T. D. \& Gascuel, O. 2018. Renewing Felsenstein's phylogenetic bootstrap in the era of big data. Nature, 556, 452-456. doi:10.1038/s41586- 018-0043-0 
Lewis, P. O. 2001. A likelihood approach to estimating phylogeny from discrete morphological character data. Systematic Biology, 50, 913-925. doi:10.1080/ 106351501753462876

Li, K., Yang, C.-Y., Liu, J. \& Wang, Z.-X. 2010. A new sauropod from the Lower Jurassic of Huili, Sichuan, China. Vertebrata PalAsiatica, 48, 185-202.

Li, K., Liu, J., Yang, C. \& Hu, F. 2011. Dinosaur assemblages from the Middle Jurassic Shaximiao Formation and Chuanjie Formation in the Sichuan-Yunnan Basin, China. Volumina Jurassica, 9, 21-42.

Li, Y., He, D., Li, D., Lu, R., Fan, C., Sun, Y. \& Huang, H. 2018. Sedimentary provenance constraints on the Jurassic to Cretaceous paleogeography of Sichuan Basin, SW China. Gondwana Research, 60, 15-33. doi:10.1016/j.gr. 2018.03.015

Liu, G., Dong, S.-W., Chen, X.-H. \& Cui, J.-J. 2017. Detrital zircon U-Pb dating of Suining Formation sandstone from the Daba Mountains, northeastern Sichuan and its stratigraphic implications. Palaeoworld, 26, 380-395. doi:10.1016/j.palwor.2017.03.002

Lu€, J., Li, S., Ji, Q., Wang, G., Zhang, J. \& Dong, Z. 2006. New eusauropod dinosaur from Yuanmou of Yunnan Province, China. Acta Geologica Sinica, 80, 1-10.

Lu€ ,J.,Li,T.,Zhong,S.,Ji,Q.\&Li,S.2008.Anew mamenchisaurid dinosaur from the Middle Jurassic of Yuanmou, Yunnan Province, China. Acta Geologica Sinica, 82, 17-26.

Lu€, J., Xu, L., Jia, S., Zhang, X., Zhang, J., Yang, L., You, H. \& Ji, Q. 2009. A new gigantic sauropod dinosaur from the Cretaceous of Ruyang, Henan, China. Geological Bulletin of China, $28,1-10$.

Lu€, J., Xu, L., Pu, H., Zhang, X., Zhang, Y., Jia, S., Chang, H., Zhang, J. \& Wei, X. 2013. A new sauropod dinosaur (Dinosauria, Sauropoda) from the late Early Cretaceous of the Ruyang Basin (central China). Cretaceous Research, 44, 202-213. doi:10.1016/j.cretres. 2013.04.009

Lull, R. S. 1919. The sauropod dinosaur Barosaurus Marsh. Memoirs of the Connecticut Academy of Arts and Sciences, 6, 1-42 p pls I-VII.

Maddison, W. P. \& Maddison, D. R. 2018. Mesquite: a modular system for evolutionary analysis. Version 3.40. Updated at: http://www.mesquiteproject.org.

Mannion, P. D. 2009. A rebbachisaurid sauropod from the Lower Cretaceous of the Isle of Wight, England. Cretaceous Research, 30, 521-526. doi:10.1016/j.cretres. 2008.09.005

Mannion, P. D. 2010. A reassessment of Mongolosaurus haplodon Gilmore, 1933, a titanosaurian sauropod dinosaur from the Early Cretaceous of Inner Mongolia, People's Republic of China. Journal of Systematic Palaeontology, 9, 355-378. doi:10.1080/14772019.2010. 527379

Mannion, P. D. \& Calvo, J. O. 2011. Anatomy of the basal titanosaur (Dinosauria, Sauropoda) Andesaurus delgadoi from the mid-Cretaceous (Albian-early Cenomanian) R1o Limay 
Formation, Neuquen Province, Argentina: implications for titanosaur systematics. Zoological Journal of the Linnean Society, 163, 155-181. doi:10.1111/j.1096- 3642.2011.00699.x

Mannion, P. D., Upchurch, P., Carrano, M. T. \& Barrett, P. M. 2011. Testing the effect of the rock record on diversity: a multidisciplinary approach to elucidating the generic richness of sauropodomorph dinosaurs through time. Biological Reviews, 86, 157-181. doi: 10.1111/j. 1469-185X.2010.00139.x

Mannion, P. D., Upchurch, P., Barnes, R. N. \& Mateus, O. 2013. Osteology of the Late Jurassic Portuguese sauropod dinosaur Lusotitan atalaiensis (Macronaria) and the evolutionary history of basal titanosauriforms. Zoological Journal of the Linnean Society, 168, 98-206. doi:10.1111/ zoj. 12029

Mannion, P. D., Allain, R. \& Moine, O. 2017. The earliest known titanosauriform sauropod dinosaur and the evolution of Brachiosauridae. PeerJ, 5, e3217. doi:10. 7717/peerj.3217

Mannion, P. D., Upchurch, P., Schwarz, D. \& Wings, O. 2019. Taxonomic affinities of the putative titanosaurs from the Late Jurassic Tendaguru Formation of Tanzania: phylogenetic and biogeographic implications for eusauropod dinosaur evolution. Zoological Journal of the Linnean Society, 185, 784-909. doi:10.1093/zoolinnean/ zly068

Marsh, A. D. \& Rowe, T. B. 2018. Anatomy and systematics of the sauropodomorph Sarahsaurus aurifontanalis from the Early Jurassic Kayenta Formation. PLoS ONE, 13, e0204007 doi: 10.1371/journal.pone.0204007

Marsh, O. C. 1878. Principal characters of American Jurassic dinosaurs. Pt. I. American Journal of Science (Series 3), 16, 411-416. doi:10.2475/ajs.s3-16.95.411

Martin, V., Buffetaut, E. \& Suteethorn, V. 1994. A new genus of sauropod dinosaur from the Sao Khua Formation (Late Jurassic or Early Cretaceous) of northeastern Thailand. Comptes Rendus de l'Academie des Sciences de Paris, 319, 1085-1092.

Martin-Rolland, V. 1999. Les sauropodes chinois. Revue de Paleobiologie, 18, 287-315.

Martınez, R. N. \& Alcober, O. A. 2009. A basal sauropodomorph (Dinosauria: Saurischia) from the Ischigualasto Formation (Triassic, Carnian) and the early evolution of Sauropodomorpha. PLoS ONE, 4, e4397. doi: 10.1371/journal.pone.0004397

Martınez, R. D. F., Lamanna, M. C., Novas, F. E., Ridgely, R. C., Casal, G. A., Martınez, J. E., Vita, J. R. \& Witmer, L. M. 2016. A basal lithostrotian titanosaur (Dinosauria: Sauropoda) with a complete skull: implications for the evolution and paleobiology of Titanosauria. PLoS ONE, 11, e0151661. doi:10.1371/ journal.pone.0151661

Mateus, O., Jacobs, L. L., Schulp, A. S., Polcyn, M. J., Tavares, T. S., Buta Neto, A., Morais, M. L. \& Antunes, M. T. 2011. Angolatitan adamastor, a new sauropod dinosaur and the first record from Angola. Anais da Academia Brasileira de Ci^encias, 83, 221-233. doi:10. 1590/ S0001-37652011000100012 
Mateus, O., Mannion, P. D. \& Upchurch, P. 2014. Zby atlanticus, a new turiasaurian sauropod (Dinosauria, Eusauropoda) from the Late Jurassic of Portugal. Journal of Vertebrate Paleontology, 34, 618-634. doi:10.1080/ 02724634.2013.822875

McIntosh, J. S. 1990. Sauropoda. Pp. 345-401 in D. B. Weishampel, P. Dodson \& H. Osmolska (eds) The Dinosauria. University of California Press, Berkeley.

McIntosh, J. S., Miller, W. E., Stadtman, K. L. \& Gillette, D. D. 1996. The osteology of Camarasaurus lewisi (Jensen, 1988). Brigham Young University Geology Studies, 41, 73-115.

McPhee, B. W., Yates, A. M., Choiniere, J. N. \& Abdala, F. 2014. The complete anatomy and phylogenetic relationships of Antetonitrus ingenipes (Sauropodiformes, Dinosauria): implications for the origins of Sauropoda. Zoological Journal of the Linnean Society, 171, 151205. doi:10.1111/zoj12127

McPhee, B. W. \& Choiniere, J. N. 2017. The osteology of Pulanesaura eocollum: implications for the inclusivity of Sauropoda (Dinosauria). Zoological Journal of the Linnean Society, 182, 830-861. doi:10.1093/zoolinnean/zlx074

Melstrom, K. M., D’Emic, M. D., Chure, D. \& Wilson, J. A. 2016. A juvenile sauropod dinosaur from the Late Jurassic of Utah, USA, presents further evidence of an avian style air-sac system. Journal of Vertebrate Paleontology, 36, e1111898. doi:10.1080/02724634.2016. 1111898

Mendez, A. 2014. The cervical vertebrae of the Late Cretaceous abelisaurid dinosaur Carnotaurus sastrei. Acta Palaeontologica Polonica, 59, 569-579. doi:10.4202/app. 2011.0129

Mo, J. 2013. Topics in Chinese dinosaur paleontology: Bellusaurus sui. Henan Science and Technology Press, Zhengzhou, 231 pp.

Mo, J., Xu, X.\& Buffetaut, E. 2010. A new eusauropod dinosaur from the Lower Cretaceous of Guangxi Province, southern China. Acta Geologica Sinica, 84, 1328-1335. doi:10.1111/j. 1755-6724.2010.00331.x

Mocho, P., Royo-Torres, R. \& Ortega, F. 2014. Phylogenetic reassessment of Lourinhasaurus alenquerensis, a basal Macronaria (Sauropoda) from the Upper Jurassic of Portugal. Zoological Journal of the Linnean Society, 170,875-916. doi:10.1111/zoj12113

Mocho, P., Royo-Torres, R. \& Ortega, F. 2019. A new macronarian sauropod from the Upper Jurassic of Portugal. Journal of Vertebrate Paleontology, 39, e1578782 doi: $10.1080 / 02724634.2019 .1578782$

Moore, A. J., Mo, J., Clark, J. M. \& Xu, X. 2018. Cranial anatomy of Bellusaurus sui (Dinosauria: Eusauropoda) from the Middle-Late Jurassic Shishugou Formation of northwest China and a review of sauropod cranial ontogeny. PeerJ, 6, e4881. doi:10.7717/peerj.4881

Nicholl, C., Mannion, P. \& Barrett, P. 2018. Sauropod dinosaur remains from a new Early Jurassic locality in the Central High Atlas of Morocco. Acta Palaeontologica Polonica, 63, 1-11, doi:10.4202/app.00425.2017 
Nylander, J. A. A., Ronquist, F., Huelsenbeck, J. P., Nieves-Aldrey, J. \& Buckley, T. 2004. Bayesian phylogenetic analysis of combined data. Systematic Biology, 53, 47-67. doi: 10.1080/10635150490264699

O'Connor, P. M. 2006. Postcranial pneumaticity: an evaluation of soft-tissue influences on the postcranial skeleton and the reconstruction of pulmonary anatomy in archosaurs. Journal of Morphology, 267, 1199-1226. doi: 10.1002/jmor.10470

O'Connor, P. M. 2007. The postcranial axial skeleton of Majungasaurus crenatissimus (Theropoda: Abelisauridae) from the Late Cretaceous of Madagascar. Memoir of the Society of Vertebrate Paleontology, 8, 127-162.

O’Reilly, J. E., Puttick, M. N., Parry, L., Tanner, A. R., Tarver, J. E., Fleming, J., Pisani, D. \& Donoghue, P. C. J. 2016. Bayesian methods outperform parsimony but at the expense of precision in the estimation of phylogeny from discrete morphological data. Biology Letters, 12, 20160081. doi:10.1098/rsbl.2016.0081

Organ, C. L. 2006. Thoracic epaxial muscles in living archosaurs and ornithopod dinosaurs. The Anatomical Record, 288A, 782-793. doi:10.1002/ar.a.20341

Osborn, H. F. \& Mook, C. C. 1921. Camarasaurus, Amphicoelias, and other sauropods of Cope. Memoirs of the American Museum of Natural History, New Series, 3, 249-387.

Otero, A. 2010. The appendicular skeleton of Neuquensaurus, a Late Cretaceous saltasaurine sauropod from Patagonia, Argentina. Acta Palaeontologica Polonica, 55, 399-426. doi:10.4202/ app.2009.0099

Otero, A. 2018. Forelimb musculature and osteological correlates in Sauropodomorpha (Dinosauria, Saurischia). PLoS ONE, 13, e0198988. doi:10.1371/journal.pone. 0198988

Ouyang, H. \& Ye, Y. 2002. The first mamenchisaurian skeleton with complete skull: Mamenchisaurus youngi. Sichuan Science and Technology Press, Chengdu, 111 pp.

Owen, R. 1842. Report on British Fossil Reptiles, Part II. Report of the British Association for the Advancement of Science, 11, 60-204.

Patterson, C. 1982. Morphological characters and homology. Pp. 21-74 in K. A. Joysey \& A. E. Friday (eds) Problems of phylogenetic reconstruction. Systematics Association Special Volume (Volume 21). Academic Press, London.

Paul, G. S. 2010. Euhelopids and mamenchisaurids. P. 178 in The Princeton field guide to dinosaurs. Princeton University Press, Princeton.

Peng, G., Ye, Y., Gao, Y., Shu, C. \& Jiang, S. 2005. Jurassic dinosaur faunas in Zigong. Sichuan People's Publishing House, Chengdu, 236 pp. 
Pi, L., Ou, Y. \& Ye, Y. 1996. A new species of sauropod from Zigong, Sichuan, Mamenchisaurus youngi. Pp. 87-91 in Anonymous (ed.) Papers on geosciences contributed to the 30th International Geological Congress. Chengdu University of Technology, Chengdu.

Pinna, M. G. G. de 1991. Concepts and tests of homology in the cladistic paradigm. Cladistics, 7, 367-394. doi:10. 1111/j.1096-0031.1991.tb00045.x

Pol, D. \& Powell, J. E. 2007. New information on Lessemsaurus sauropoides (Dinosauria: Sauropodomorpha) from the Upper Triassic of Argentina. Special Papers in Palaeontology, 77, 223-243.

Pol., D. \& Escapa, I. H. 2009. Unstable taxa in cladistic analysis: identification and the assessment of relevant charaters. Cladistics, 25, 515-527. doi:10.1111/j.10960031.2009.00258.x

Poropat, S. F., Upchurch, U., Mannion, P. D., Hocknull, S. A., Kear, B. P., Sloan, T., Sinapius, G. H. K. \& Elliot, D. A. 2015. Revision of the sauropod dinosaur Diamantinasaurus matildae Hocknull et al. 2009 from the mid-Cretaceous of Australia: Implications for Gondwanan titanosauriform dispersal. Gondwana Research, 27, 995-1033.

Poropat, S. F., Mannion, P. D., Upchurch, P., Hocknull, S. A., Kear, B. P., Kundrat, M., Tischler, T. R., Sloan, T., Sinapius, G. H. K., Elliott, J. A. \& Elliott, D. A. 2016. New Australian sauropods shed light on Cretaceous dinosaur palaeobiogeography. Scientific Reports, 6, 34467. doi: $10.1038 /$ srep34467

Powell, J. E. 1987. Morfologia del esqueleto axial de los dinosaurios titanosauridos (Saurischia, Sauropoda) del Estado de Minas Gerais, Brasil. Pp. 155-171 in Anonymous (ed.) Anais do X Congreso Brasileiro de Paleontologia, Volume 1. congreso Brasileiro de paleontologia, Rio de Janeiro.

Powell, J. E. 2003. Revision of South American titanosaurid dinosaurs: palaeobiological, palaeobiogeographical, and phylogenetic aspects. Records of the Queen Victoria Museum, 111, $1-173$.

Puttick, M. N., O’Reilly, J. E., Tanner, A. R., Fleming, J. F., Clark, J., Holloway, L., LozanoFernandez, J., Parry, L. A., Tarver, J. E., Pisani, D. \& Donoghue, P. C. J. 2017. Uncertain-tree: discriminating among competing approaches to the phylogenetic analysis of phenotypic data. Proceedings of the Royal Society B, 284, 20162290 doi:10.1098/rspb.2016.2290

Pyron, R. A. 2016. Novel approaches for phylogenetic inference from morphological data and total-evidence dating in squamate reptiles (lizards, snakes, and amphisbaenians). Systematic Biology, 66, 38-56. doi:10. 1093/sysbio/syw068

Raath, M. A. 1972. Fossil vertebrate studies in Rhodesia: a new dinosaur (Reptilia: Saurischia) from near the Trias-Jurassic boundary. Arnoldia, 5(30), 1-37. 
Racey, A. \& Goodall, J. G. S. 2009. Palynology and stratigraphy of the Mesozoic Khorat Group red bed sequences of Thailand. Pp. 69-83 in E. Buffetaut, G. Cuny, J. Le Loeuff \& V. Suteethorn (eds) Late Palaeozoic and Mesozoic ecosystems in SE Asia. Geological Society of London, Special Publication, 315.

Rambaut, A., Drummond, A. J., Xie, D., Baele, G. \& Suchard, M. A. 2018. Posterior summarization in Bayesian phylogenetics using Tracer 1.7. Systematic Biology, 67, 901-904. doi:10.1093/sysbio/syy032

Remes, K. 2007. A second Gondwana diplodocid dinosaur from the Upper Jurassic Tendaguru Beds of Tanzania, East Africa. Palaeontology, 50, 653-667.

Remes, K. 2008. Evolution of the pectoral girdle and forelimb in Sauropodomorpha (Dinosauria, Saurischia): osteology, myology and function. Unpublished $\mathrm{PhD}$ thesis, Ludwig- MaximiliansUniversit $€$ at $M €$ unchen, $355 \mathrm{pp}$.

Remes, K., Ortega, F., Fierro, I., Joger, U., Kosma, R., Ferrer, J. M. M., for the Project Paldes, for the Niger Project SNHM, Ide, O. A. \& Maga, A. 2009. A new basal sauropod dinosaur from the Middle Jurassic of Niger and the early evolution of Sauropoda. PLoS ONE, 4, e6924. doi: 10.1371/journal.pone.0006924

Ren, X.-X., Huang, J.-D. \& You, H.-L. 2018. The second mamenchisaurid dinosaur from the Middle Jurassic of Eastern China. Historical Biology. doi:10.1080/08912963. 2018.1515935

Rieppel, O. \& Kearney, M. 2002. Similarity. Biological Journal of the Linnean Society, 75, 5982. doi: $10.1046 / j .1095-8312.2002 .00006 . x$

Ronquist, F., Klopfstein, S., Vilhelmsen, L., Schulmeister, S., Murray, D. L. \& Rasnitsyn, A. P. 2012. A total- evidence approach to dating with fossils, applied to the early radiation of the Hymenoptera. Systematic Biology, 61, 973-999. doi:10.1093/sysbio/sys058

Royo-Torres, R., Cobos, A. \& Alcala, L. 2006. A giant European dinosaur and a new sauropod clade. Science, 314, 1925-1927. doi:10.1126/science.1132885

Royo-Torres, R., Cobos, A., Luque, L., Aberasturi, A., Espılez, E., Fierro, I., Gonzalez, A., Mampel, L. \& Alcala, L. 2009. High European sauropod dinosaur diversity during JurassicCretaceous transition in Riodeva (Teruel, Spain). Palaeontology, 52, 1009-1027. doi:10. 1111/j. 1475-4983.2009.00898.x

Royo-Torres, R. \& Upchurch, P. 2012. The cranial anatomy of the sauropod Turiasaurus riodevensis and implications for its phylogenetic relationships. Journal of Systematic Palaeontology, 10, 553-583. doi:10.1080/14772019.2011. 598577

Royo-Torres, R., Alcala, L. \& Cobos, A. 2012. A new specimen of the Cretaceous sauropod Tastavinsaurus sanzi from El Castellar (Teruel, Spain), and a phylogenetic analysis of the Laurasiformes. Cretaceous Research, 34, 61-83. doi:10.1016/j.cretres.2011.10.005 
Royo-Torres, R., Upchurch, P., Kirkland, J. I., DeBlieux, D. D., Foster, J. R., Cobos, A. \& Alcala, L. 2017. Descendants of the Jurassic turiasaurs from Iberia found refuge in the Early Cretaceous of western USA. Scientific Reports, 7, 14311. doi:10.1038/s41598-017-14677-2

Russell, D. A. 1993. The role of Central Asia in dinosaurian biogeography. Canadian Journal of Earth Sciences, 30, 2002-2012. doi:10.1139/e93-176

Russell, D. A. \& Zheng, Z. 1993. A large mamenchisaurid from the Junggar Basin, Xinjiang, People's Republic of China. Canadian Journal of Earth Sciences, 30, 2082-2095. doi:10.1139/ e93-180

Salgado, L., Coria, R. A. \& Calvo, J. O. 1997. Evolution of titanosaurid sauropods. I: phylogenetic analysis based on the postcranial evidence. Ameghiniana, 34, 3-32.

Salgado, L. \& de Souza Carvalho, I. 2008. Uberabatitan ribeiroi, a new titanosaur from the Marlia Formation (Bauru Group, Upper Cretaceous), Minas Gerais, Brazil. Palaeontology, 51, 881-901. doi:10.1111/j.1475-4983.2008.00781.x

Schmidt-Lebuhn, A. N. 2016. TNT script for the Templeton test. doi:10.13140/RG.2.1.3484.9522

Schwarz, D. \& Fritsch, G. 2006. Pneumatic structures in the cervical vertebrae of the Late Jurassic Tendaguru sauropods Brachiosaurus brancai and Dicraeosaurus. Eclogae Geologicae Helvetiae, 99, 65-78. doi:10.1007/ s00015-006-1177-X

Schwarz, D., Frey, E. \& Meyer, C. A. 2007. Pneumaticity and soft-tissue reconstructions in the neck of diplodocid and dicraeosaurid sauropods. Acta Palaeontologica Polonica, 52, 167-188.

Schwarz-Wings, D. 2009. Reconstruction of the thoracic epaxial musculature of diplodocid and dicraeosaurid sauropods. Journal of Vertebrate Paleontology, 29, 517-534. doi:

10.1671/039.029.0229

Seeley, H. G. 1887. On the classification of the fossil animals commonly named Dinosauria. Proceedings of the Royal Society of London, 43, 165-171.

Sekiya, T. 2011. Re-examination of Chuanjiesaurus anaensis (Dinosauria: Sauropoda) from the Middle Jurassic Chuanjie Formation, Lufeng County, Yunnan Province, Southwest China. Memoir of the Fukui Prefectural Dinosaur Museum, 10, 1-54.

Sereno, P. C. 1990. Psittacosauridae. Pp. 579-592 in D. B. Weishampel, P. Dodson \& H. Osmolska (eds) The Dinosauria. University of California Press, Berkeley.

Sereno, P. C., Beck, A. L., Dutheil, D. B., Larsson, H. C. E., Lyon, G. H., Moussa, B., Sadleir, R. W., Sidor, C. A., Varricchio, D. J., Wilson, G. P. \& Wilson, J. A. 1999. Cretaceous sauropods from the Sahara and the uneven rate of skeletal evolution among dinosaurs. Science, 286, 13421347. doi:10.1126/science.286.5443. 1342 
Sereno, P. C., Wilson, J. A., Witmer, L. M., Whitlock, J. A., Maga, A., Ide, O. \& Rowe, T. A. 2007. Structural extremes in a Cretaceous dinosaur. PLoS ONE, 2, e1230. doi:10.1371/ journal.pone. 0001230

Silva Junior, J. C. G., Marinho, T. S., Martinelli, A. G. \& Langer, M. C. 2019. Osteology and systematics of

Uberabatitan ribeiroi (Dinosauria; Sauropoda): a Late Cretaceous titanosaur from Minas Gerais, Brazil. Zootaxa, 3, 401-328.

Simões, T. R., Caldwell, M. W., Palci, A. \& Nydam, R. L. 2017. Giant taxon-character matrices: quality of character constructions remains critical regardless of size. Cladistics, 33, 198-219.

Stadler, T. 2010. Sampling-through-time in birth-death trees. Journal of Theoretical Biology, 267, 396-404. doi:10. 1016/j.jtbi.2010.09.010

Stevens, K. A. \& Parrish, J. M. 1999. Neck posture and feeding habits of two Jurassic sauropod dinosaurs. Science, 284, 798-800. doi:10.1126/science.284.5415.798

Stevens, K. A. \& Parrish, J. M. 2005. Digital reconstructions of sauropod dinosaurs and implications for feeding. Pp. 178-200 in K. Curry Rogers \& J. A. Wilson (eds) The sauropods: evolution and paleobiology. University of California Press, Berkeley.

Suteethorn, S., Le Loeuff, J., Buffetaut, E., Suteethorn, V. \& Wongko, K. 2013. First evidence of a mamenchisaurid dinosaur from the Upper Jurassic-Lower Cretaceous Phu Kradung Formation of Thailand. Acta Palaeontologica Polonica, 58, 459-469. doi:10.4202/app.2009.0155

Tan, C., Dai, H., He, J.-J., Zhang, F., Hu, X.-F., Yu, H.-D., Li, N., Wei, G.-B., Peng, G.-Z., Ye, Y., Zhang, Q.-N., Ren, X.-X. \& You, H.-L. 2019. Discovery of Omeisaurus (Dinosauria: Sauropoda) in the Middle Jurassic Shaximiao Formation of Yunyang, Chongqing, China. Vertebrata Palasiatica, 57, 105-116, doi: 10.19615/j.cnki.1000-3118. 181115.

Tan, C., Xiao, M., Dai, H., Hu, X.-F., Li, N., Ma, Q.-Y., Wei, Z.-Y., Yu, H. D., Xiong, C., Peng, G.-Z., Jiang, S., Ren, X.-X. \& You, H-L. 2020. A new species of Omeisaurus (Dinosauria:

Sauropoda) from the Middle Jurassic of Yunyang, Chongqing, China. Historical Biology. doi: 10.1080/08912963.2020.1743286

Tang, F., Jin, X., Kang, X. \& Zhang, G. 2001. Omeisaurus maoianus, a complete Sauropoda from Jingyan, Sichuan. Research Works of Natural History Museum of Zhejiang, 10, 1-127.

Taylor, M. P., Wedel, M. J. \& Cifelli, R. L. 2011. A new sauropod dinosaur from the Lower Cretaceous Cedar Mountain Formation, Utah, USA. Acta Palaeontologica Polonica, 56, 75-98. doi:10.4202/app.2010.0073

Taylor, M. P. \& Wedel, M. J. 2013. Why sauropods had long necks; and why giraffes have short necks. PeerJ, 1, e36. doi:10.7717/peerj.36 
Templeton, A. R. 1983. Phylogenetic inference from restriction endonuclease cleavage site maps with particular reference to the evolution of humans and the apes. Evolution, 37, 221-244. doi: $10.2307 / 2408332$

Tsai, C.-H. \& Fordyce, R. E. 2014. Juvenile morphology in baleen whale phylogeny. Naturwissenschaften, 101, 765-769. doi:10.1007/s00114-014-1216-9

Tschopp, E. \& Mateus, O. 2013. The skull and neck of a new flagellicaudatan sauropod from the Morrison Formation and its implication for the evolution and ontogeny of diplodocid dinosaurs. Journal of Systematic Palaeontology, 11, 853-888. doi:10.1080/14772019.2012. 746589

Tschopp, E., Mateus, O. \& Benson, R. B. J. 2015a. A specimen-level phylogenetic analysis and taxonomic revision of Diplodocidae (Dinosauria, Sauropoda). PeerJ, 3, e857. doi:10.7717/peerj. 857

Tschopp, E., Wings, O., Frauenfelder, T. \& Brinkmann, W. 2015b. Articulated bone sets of manus and pedes of Camarasaurus (Sauropoda, Dinosauria). Palaeontologia Electronica, 18(2), 44A. doi:10.26879/559

Tschopp, E. 2016. Nomenclature of vertebral laminae in lizards, with comments on ontogenetic and serial variation in Lacertini (Squamata, Lacertidae). PLoS ONE, 11, e0149445. doi:10.1371/ journal.pone.0149445

Tschopp, E. \& Mateus, O. 2017. Osteology of Galeamopus pabsti sp. nov. (Sauropoda: Diplodocidae), with implications for neurocentral closure timing, and the cervico-dorsal transition in diplodocids. PeerJ, 5, e3179. doi:10.7717/peerj.3179

Tschopp, E. \& Upchurch, P. 2019. The challenges and potential utility of phenotypic specimenlevel phylogeny based on maximum parsimony. Earth and Environmental Science Transactions of the Royal Society of Edinburgh, 109, 301-323. doi:10.1017/S1755691018000877

Tsuihiji, T. 2005. Homologies of the transversospinalis muscles in the anterior presacral region of Sauria (crown Diapsida). Journal of Morphology, 263, 151-178. doi:10. 1002/jmor.10294

Tsuihiji, T. 2007. Homologies of the longissimus, iliocostalis, and hypaxial muscles in the anterior presacral region of extant Diapsida. Journal of Morphology, 268, 986-1020. doi: 10.1002/jmor.10565

Tykoski, R. S. \& Fiorillo, A. R. 2016. An articulated cervical series of Alamosaurus sanjuanensis Gilmore, 1922 (Dinosauria, Sauropoda) from Texas: new perspective on the relationships of North America's last giant sauropod. Journal of Systematic Palaeontology, 15, 339-364. doi:10. 1080/14772019.2016.1183150

Upchurch, P. 1995. The evolutionary history of sauropod dinosaurs. Philosophical Transactions of the Royal Society B, 349, 365-390. doi:10.1098/rstb.1995.0125

Upchurch, P. 1998. The phylogenetic relationships of sauropod dinosaurs. Zoological Journal of the Linnean Society, 124, 43-103. doi:10.1111/j.1096-3642.1998. tb00569.x 
Upchurch, P. 1999. The phylogenetic relationships of the Nemegtosauridae (Saurischia, Sauropoda). Journal of Vertebrate Paleontology, 19, 106-125. doi:10.1080/

02724634.1999 .10011127

Upchurch, P. \& Martin, J. 2002. The Rutland Cetiosaurus: the anatomy and relationships of a Middle Jurassic British sauropod dinosaur. Palaeontology, 45, 1049-1074. doi:10.

$1111 / 1475-4983.00275$

Upchurch, P., Hunn, C. A. \& Norman, D. B. 2002. An analysis of dinosaurian biogeography: evidence for the existence of vicariance and dispersal patterns caused by geological events. Proceedings of the Royal Society of London, Series B, 269, 613-621. doi:10.1098/rspb.2001. 1921

Upchurch, P. \& Martin, J. 2003. The anatomy and taxonomy of Cetiosaurus (Saurischia, Sauropoda) from the Middle Jurassic of England. Journal of Vertebrate Paleontology, 23, 208231.

Upchurch, P., Barrett, P. M. \& Dodson, P. 2004. Sauropoda. Pp. 259-322 in D. B. Weishampel, P. Dodson \& H. Osmolska (eds) The Dinosauria. Second Edition. University of California Press, Berkeley.

Upchurch, P. \& Barrett, P. M. 2005. Phylogenetic and taxic perspectives on sauropod diversity. Pp. 104-124 in K. A. Curry Rogers \& J. A. Wilson (eds) The sauropods: evolution and paleobiology. University of California Press, Berkeley.

Upchurch, P., Mannion, P. D. \& Barrett, P. M. 2011. Sauropod dinosaurs. Pp. 476-525 in D. J. Batten (ed.) Field guide to English Wealden fossils. Palaeontological Association, London.

Upchurch, P., Mannion, P. D. \& Taylor, M. P. 2015. The anatomy and phylogenetic relationships of 'Pelorosaurus' becklesii (Neosauropoda, Macronaria) from the Early Cretaceous of England. PLoS ONE, 10, e0125819. doi:10. 1371/journal.pone.0125819

Wang, J., Ye, Y., Pei, R., Tian, Y., Feng, C., Zheng, D. \& Chang, S.-C. 2018. Age of Jurassic basal sauropods in Sichuan, China: a reappraisal of basal sauropod evolution. Geological Society of America Bulletin, 130, 1493-1500. doi:10.1130/B31910.1

Wang, J., Norell, M. A., Pei, R., Ye, Y. \& Chang, S.-C. 2019. Surprisingly young age for the mamenchisaurid sauropods in South China. Cretaceous Research, 104, 104176.

Wang, S., Stiegler, J., Amiot, R., Wang, X., Du, G., Clark, J. M. \& Xu, X. 2017. Extreme ontogenetic changes in a ceratosaurian theropod. Current Biology, 27, 144-148. doi: 10.1016/ j.cub.2016.10.043

Wang, X., You, H., Meng, Q., Gao, C., Cheng, X. \& Liu, J. 2007. Dongbeititan dongi, the first sauropod dinosaur from the Lower Cretaceous Jehol Group of Western Liaoning Province, China. Acta Geologica Sinica, 81, 911-916. 
Wedel, M. J. 2003. The evolution of vertebral pneumaticity in sauropod dinosaurs. Journal of Vertebrate Paleontology, 23, 344-357. doi:10.1671/0272-

4634(2003)023[0344:TEOVPI]2.0.CO;22.0.CO;2]

Wedel, M. J. 2006. Pneumaticity, neck length, and body size in sauropods. Journal of Vertebrate Paleontology, 26(3, Suppl.), 137A.

Wedel, M. J. 2009. Evidence for bird-like air sacs in saurischian dinosaurs. Journal of Experimental Zoology 311A, 611-628. doi: 10.1002/jez.513

Wedel, M. J., Cifelli, R. L. \& Sander, R. K. 2000. Osteology, paleobiology, and relationships of the sauropod dinosaur Sauroposeidon. Acta Paleontologica Polonica, 45, 343-388.

Wedel, M. J. \& Sanders, R. K. 2002. Osteological correlates of cervical musculature in Aves and Sauropoda (Dinosauria: Saurischia), with comments on the cervical ribs of Apatosaurus.

PaleoBios, 22, 1-6.

Wedel, M. J. \& Taylor, M. P. 2013a. Neural spine bifurcation in sauropod dinosaurs from the Morrison Formation: ontogenetic and phylogenetic implications. PalArch's Journal of Vertebrate Palaeontology, 10, 1-34.

Wedel, M. J. \& Taylor, M. P. 2013b. Caudal pneumaticity and pneumatic hiatuses in the sauropod dinosaurs Giraffatitan and Apatosaurus. PLoS ONE, 8, e78213. doi: 10.1371/ journal.pone. 0078213

Weishampel, D. B., Barrett, P. M., Coria, R. A., Le Loeuff, J., Xu, X., Zhao, X., Sahni, A., Gomani, E. M. \& Noto, C. R. 2004. Dinosaur distribution. Pp. 517-606 in D. B. Weishampel, P. Dodson \& H. Osmolska (eds) The Dinosauria. Second Edition. University of California Press, Berkeley.

Whitlock, J. A. 2011. Re-evaluation of Australodocus bohetii, a putative diplodocoid sauropod from the Tendaguru Formation of Tanzania, with comment on Late Jurassic sauropod faunal diversity and palaeoecology. Palaeogeography, Palaeoclimatology, Palaeoecology, 309, 333-341. doi:10.1016/j.palaeo.2011.07.001

Wilson, J. A. 1999. A nomenclature for vertebral laminae in sauropods and other saurischian dinosaurs. Journal of Vertebrate Paleontology, 19, 639-653. doi:10.1080/

02724634.1999 .10011178

Wilson, J. A. 2002. Sauropod dinosaur phylogeny: critique and cladistic analysis. Zoological Journal of the Linnean Society, 136, 215-275. doi:10.1046/j.1096-3642.2002. 00029.x

Wilson, J. A. 2011. Anatomical terminology for the sacrum of sauropod dinosaurs. Contributions from the Museum of Paleontology, University of Michigan, 32, 59-69.

Wilson, J. A. 2012. New vertebral laminae and patterns of serial variation in vertebral laminae of sauropod dinosaurs. Contributions from the Museum of Paleontology, University of Michigan, $32,91-110$. 
Wilson, J. A. \& Sereno, P. C. 1998. Early evolution and higher-level phylogeny of sauropod dinosaurs. Memoir of the Society of Vertebrate Paleontology, 5, 1-79. doi:10.

1080/02724634.1998.10011115

Wilson, J. A. \& Upchurch, P. 2003. A revision of Titanosaurus Lydekker (Dinosauria Sauropoda), the first dinosaur genus with a 'Gondwanan' distribution. Journal of Systematic Palaeontology, 1, 125-160. doi:10.1017/ S1477201903001044

Wilson, J. A. \& Upchurch, P. 2009. Redescription and reassessment of the phylogenetic affinities of Euhelopus zdanskyi (Dinosauria: Sauropoda) from the Early Cretaceous of China. Journal of Systematic Palaeontology, 7, 199-239. doi:10.1017/S1477201908002691

Wilson, J. A., D’Emic, M. D., Ikejiri, T., Moacdieh, E. M. \& Whitlock, J. A. 2011. A nomenclature for vertebral fossae in sauropods and other saurischian dinosaurs. PLoS ONE, 6 , e17114. doi:10.1371/journal.pone.0017114

Wilson, J. A., Mohabey, D. M., Lakra, P. \& Bhadran, A. 2019. Titanosaur (Dinosauria: Sauropoda) vertebrae from the Upper Cretaceous Lameta Formation of western and central India. Contributions from the Museum of Paleontology University of Michigan, 33, 1-27.

Wiman, C. 1929. Die Kreide-Dinosaurier aus Shantung. Palaeontologia Sinica (Series C), 6, 167.

Wings, O., Schwarz-Wings, D. \& Fowler, D. W. 2011. New sauropod material from the Late Jurassic part of the Shishugou Formation (Junggar Basin, Xinjiang, NW China). Neues Jahrbuch f€ur Geologie und Pal€aontologie, Abhandlungen, 262, 129-150. doi:

$10.1127 / 0077-7749 / 2011 / 0183$

Woodruff, D. C. 2017. Nuchal ligament reconstructions in diplodocid sauropods support horizontal neck feeding postures. Historical Biology, 29, 308-319. doi:10.1080/

08912963.2016.1158257

Woodruff, D. C. 2014. The anatomy of the bifurcated neural spine and its occurrence within Tetrapoda. Journal of Morphology, 275, 1053-1065. doi:10.1002/jmor.20283

Woodruff, D. C. \& Fowler, D. W. 2012. Ontogenetic influence on neural spine bifurcation in Diplodocoidea (Dinosauria: Sauropoda): a critical phylogenetic character. Journal of Morphology, 273, 754-764. doi:10.1002/jmor.20021

Woodruff, D. C., Atwood, N. J. \& Madill, A. 2016. The structural preservation of a titanosaurid (Dinosauria: Sauropoda) vertebral ligament. Cretaceous Research, 60, 253-266. doi:10.1016/ j.cretres.2015.12.009 Woodward, A. S. 1905. On parts of the skeleton of Cetiosaurus leedsi, a sauropodous dinosaur from the Oxford Clay of Peterborough. Proceedings of the Zoological Society of London, 1, 232-243. 
Wright, A. M., \& Hillis, D. M. 2014. Bayesian analysis using a simple likelihood model outperforms parsimony for estimation of phylogeny from discrete morphological data. PLoS ONE, 9, e109210 doi:10.1371/journal.pone.0109210

Wu, W., Zhou, C., Wings, O., Sekiya, T. \& Dong, Z. 2013. A new gigantic sauropod dinosaur from the Middle Jurassic of Shanshan, Xinjiang. Global Geology, 32, 437-446.

Xie, W., Lewis, P. O., Fan, Y., Kuo, L. \& Chen, M.-H. 2011. Improving marginal likelihood estimation for Bayesian phylogenetic model selection. Systematic Biology, 60, 150-160. doi: 10.1093/sysbio/syq085

Xing, L., Miyashita, T., Currie, P. J., You, H., Zhang, J. \& Dong, Z. 2013. A new basal eusauropod from the Middle Jurassic of Yunnan, China, and faunal compositions and transitions of Asian sauropodomorph dinosaurs. Acta Palaeontologica Polonica, 60, 145-154. doi:10.4202/ app. 2012.0151

Xing, L., Miyashita, T., Zhang, J., Li, D., Ye, Y., Sekiya, T., Wang, F. \& Currie, P. J. 2015. A new sauropod dinosaur from the Late Jurassic of China and the diversity, distribution, and relationships of mamenchisaurids. Journal of Vertebrate Paleontology, 35, e889701. doi:10. 1080/02724634.2014.889701

Xu, J. \& Li, Z. 2015. Middle-Late Mesozoic sedimentary provenances of the Luxi and Jiaolai areas: implications for tectonic evolution of the North China Block. Journal of Asian Earth Sciences, 111, 284-301. doi:10.1016/j.jseaes. 2015.07.008

Xu, X., Upchurch, P., Mannion, P. D., Barrett, P. M., Regalado-Fernandez, O. R., Mo, J., Ma, J. \& Liu, H. 2018. A new Middle Jurassic diplodocoid suggests an earlier dispersal and diversification of sauropod dinosaurs. Nature Communications, 9, 2700. doi:10.1038/s41467018-05128-1

Yates, A. M. \& Kitching, J. W. 2003. The earliest known sauropod dinosaur and the first steps towards sauropod locomotion. Proceedings of the Royal Society of London B, 270, 1753-1758. doi:10.1098/ rspb.2003.2417

Ye, Y., Ouyang, H. \& Fu, Q.-M. 2001. New material of Mamenchisaurus hochuanensis from Zigong, Sichuan. Vertebrata PalAsiatica, 39, 266-271.

Ye, Y., Gao, Y.-H. \& Jiang, S. 2005. A new genus of sauropod from Zigong, Sichuan. Vertebrata PalAsiatica, 43, 175-181.

Yeh, C. H. 1975. Jurassic system. Pp. 11-30 in Z. Su (ed.) Mesozoic redbeds of Yunnan. Geological Publishing House, Beijing.

You, H., Tang, F. \& Luo, Z. 2003. A new basal titanosaur (Dinosauria: Sauropoda) from the Early Cretaceous of China. Acta Geologica Sinica, 77, 424-429. 
You, H., Li, D., Zhou, L. \& Ji, Q. 2006. Huanghetitan liujiaxiaensis, a New Sauropod Dinosaur from the Lower Cretaceous Hekou Group of Lanzhou Basin, Gansu Province, China. Geological Review, 52, 668-674.

You, H., Li, D., Zhou, L. \& Ji, Q. 2008. Daxiatitan binglingi: a giant sauropod dinosaur from the Early Cretaceous of China. Gansu Geology, 17, 1-17.

Young, C.-C. 1935. Dinosaurian remains from Mengyin, Shantung. Bulletin of the Geological Society of China, 15, 519-533. doi:10.1111/j.1755-6724.1935.mp14004004.x

Young, C.-C. 1937. A new dinosaurian from Sinkiang. Palaeontologia Sinica (series C), 2, 1-25.

Young, C.-C. 1939. On a new Sauropoda, with notes on other fragmentary reptiles from

Szechuan. Bulletin of the Geological Society of China, 19, 279-315. doi:10.1111/j.

1755-6724.1939.mp19003005.x

Young, C.-C. 1954. On a new sauropod from Yiping, Szechuan, China. Scientia Sinica, 3, 491505.

Young, C.-C. 1958. New sauropods from China. Vertebrata PalAsiatica, 2, 1-28.

Young, C.-C. \& Zhao, X. 1972. [Mamenchisaurus hochuanensis, sp. nov.]. Academica Sinica, 8, 10-30. [In Chinese.]

Zaher, H., Pol, D., Carvalho, A. B., Nascimento, P. M., Riccomini, C., Larson, P., Juarez-Valieri, R., Pires- Domingues, R., Jr, N. J. da S. \& Campos, D. de A. 2011. A complete skull of an Early Cretaceous sauropod and the evolution of advanced titanosaurians. PLoS ONE, 6, e16663. doi: 10.1371/journal.pone.0016663

Zhang, C., Stadler, T., Klopfstein, S., Heath, T. A. \& Ronquist, F. 2016. Total-evidence dating under the fossilized birth-death process. Systematic Biology, 65, 228-249. doi:10.1093/sysbio/ syv080

Zhang, X., Lu€, J., Xu, L., Yang, L., Hu, W., Jia, S., Ji, Q. \& Zhang, C. 2009. A new sauropod from the Late Cretaceous Gaogou Formation of Nanyang, Henan Province. Acta Geologica Sinica, 83, 212-221. doi:10. 1111/j.1755-6724.2009.00032.x

Zhang, X.-Q., Li, D.-Q., Xie, Y. \& You, H.-L. 2018. Redescription of the cervical vertebrae of the mamenchisaurid Sauropod Xinjiangtitan shanshanesis Wu et al. 2013. Historical Biology. doi:10.1080/08912963. 2018.1539970

Zhang, Y. 1988. The Middle Jurassic dinosaur fauna from Dashanpu, Zigong, Sichuan, Volume 1. Sauropod dinosaur (I): Shunosaurus. Sichuan Publishing House of Science and Technology, Chengdu, 89 pp. [In Chinese, with English summary.]

Zhang, Y., Li, K. \& Zeng, Q. 1998. A new species of sauropod from the Late Jurassic of the Sichuan Basin (Mamenchisaurus jingyanensis sp. nov.). Journal of the Chengdu University of Technology, 25, 61-68. [In Chinese, with English summary.] 
Zhao, X. 1993. A new Middle Jurassic sauropod subfamily (Klamelisaurinae subfam. nov.) from Xinjiang Autonomous Region, China. Vertebrata PalAsiatica, 31, 132-138. [In Chinese, with English summary.]

Zhou, C., Wu, W., Sekiya, T. \& Dong, Z. 2018. A new Titanosauriformes dinosaur from Jehol Biota of western Liaoning, China. Global Geology, 37, 327-333.

Zurriaguz, V. \& Powell, J. 2015. New contributions to the presacral osteology of Saltasaurus loricatus (Sauropoda, Titanosauria) from the Upper Cretaceous of northern Argentina.

Cretaceous Research, 54, 283-300. doi:10. 1016/j.cretres.2014.12.012 
Table 1. Dimensions of cervical vertebrae of Klamelisaurus gobiensis (IVPP V9492). Cervical position is approximated (see text). Values are given in millimetres. Underlined values indicate the element is incompletely preserved, and italicized values are approximations. Abbreviations: DPW, width across diapophyses; fEI, functional elongation index; FL, functional length; MLW, midlength width of centrum; NSL, neural spine length at dorsal end; PCH, posterior centrum height; PCW, posterior centrum width; TH, maximum midline height of vertebra.

\begin{tabular}{|c|c|c|c|c|c|c|c|c|}
\hline Vertebra & FL & $\mathrm{PCH}$ & PCW & DPW & NSL & TH & MLW & fEI \\
\hline ' 9 ' & 435 & 120 & 160 & - & - & 280 & - & 3.1 \\
\hline '10' & 420 & 125 & 150 & 225 & 69 & 350 & 104 & 3.1 \\
\hline '11' & 435 & 161 & 187 & 240 & 89 & 360 & - & 2.5 \\
\hline '12' & 403 & 166 & 192 & $\underline{263}$ & 62 & 365 & 125 & 2.3 \\
\hline '13' & 410 & 173 & 185 & 281 & 72 & 380 & 123 & 2.3 \\
\hline '14' & 405 & 206 & 191 & 303 & 65 & 390 & 136 & 2.0 \\
\hline ‘15’ & 430 & 214 & 202 & 320 & 74 & 470 & 131 & 2.1 \\
\hline '16' & 340 & 220 & 206 & 370 & - & 430 & - & 1.6 \\
\hline
\end{tabular}


Table 2. Dimensions of dorsal vertebrae and dorsosacral 1 of Klamelisaurus gobiensis (IVPP V9492). Values are given in millimetres. Underlined values indicate the element is incompletely preserved, and italicized values indicate approximations. Abbreviations: DPW, width across diapophyses; FL, functional length; MLW, midlength width; NSL, neural spine length at dorsal end; PCH, posterior centrum height; PCW, posterior centrum width; TH, maximum midline height of vertebra; WPOZ, width across outer edges of postzygapophyses.

\begin{tabular}{|c|c|c|c|c|c|c|}
\hline Vertebra & FL & PCH & PCW & DPW & WPOZ & TH \\
\hline 1 & 267 & 210 & 222 & $\underline{392}$ & 197 & 430 \\
\hline 2 & 237 & 234 & 183 & $\underline{422}$ & - & 490 \\
\hline 3 & 191 & 217.5 & 178 & - & 158 & 534 \\
\hline 4 & 170 & 219 & 190 & - & 151 & 538 \\
\hline 5 & 160 & 230 & 181 & - & 146 & 520 \\
\hline 6 & 172 & 224 & 185 & 380 & 144 & 550 \\
\hline 7 & 165 & 235 & 185 & $\underline{410}$ & 127 & 570 \\
\hline 8 & 140 & 212 & 183 & 350 & 128 & 640 \\
\hline 9 & 184 & 249 & 169 & 325 & 127 & 670 \\
\hline 10 & 159 & 248 & 188 & 325 & $\underline{112}$ & 680 \\
\hline 11 & 178 & 255 & 191 & - & 127 & 710 \\
\hline 12 & 146 & 224 & - & - & 113 & 698 \\
\hline Dorsosacral 1 & 163 & 215 & - & - & 77 & 694 \\
\hline
\end{tabular}


Table 3. Functional lengths of sacral vertebrae of Klamelisaurus gobiensis (IVPP V9492). Values are given in millimetres. Italicized values indicate approximations.

\begin{tabular}{|c|c|c|c|c|c|c|}
\hline Vertebra & $\mathbf{1}$ & $\mathbf{2}$ & $\mathbf{3}$ & $\mathbf{4}$ & $\mathbf{5}$ & $\mathbf{6}$ \\
\hline $\begin{array}{c}\text { Functional } \\
\text { length }\end{array}$ & 163 & 163 & 105 & 105 & 105 & 147 \\
\hline
\end{tabular}


Table 4. Dimensions of the neural spines of anteriormost and anterior caudal vertebrae $(\mathrm{Ca})$ of Klamelisaurus gobiensis (IVPP V9492). Values given in millimetres. Underlined values indicate the element is incompletely preserved and italicized values indicate approximations.

\begin{tabular}{|c|c|c|c|c|c|}
\hline & $\begin{array}{l}\text { Total neural } \\
\text { spine height }\end{array}$ & $\begin{array}{l}\text { Proximal } \\
\text { width }\end{array}$ & $\begin{array}{l}\text { Distal } \\
\text { maximum } \\
\text { width }\end{array}$ & $\begin{array}{l}\text { Proximal } \\
\text { anteroposterior } \\
\text { length }\end{array}$ & $\begin{array}{l}\text { Distal } \\
\text { anteroposterior } \\
\text { length }\end{array}$ \\
\hline Ca1 & $\underline{188}$ & $\underline{57}$ & 76 & $\underline{58}$ & 55 \\
\hline $\mathrm{Ca} 2$ & 195 & 50 & 67 & 52 & 59 \\
\hline Ca3 & 200 & 54 & 59 & 59 & 57 \\
\hline $\mathrm{Ca} 4$ & 205 & 45 & 56 & 47 & 59 \\
\hline Ca6 & $\underline{101}$ & $\underline{36}$ & 49 & $\underline{44}$ & 55 \\
\hline Ca8 & $\underline{153}$ & $\underline{41}$ & 54 & $\underline{44}$ & 54 \\
\hline Ca9 & $\underline{76}$ & - & 42 & - & 52 \\
\hline Ca10 & $\underline{67}$ & - & 50 & - & 48 \\
\hline Ca11 & - & - & 44 & - & 50 \\
\hline
\end{tabular}


Table 5. Dimensions of the centra of the middle to posterior caudal vertebrae of Klamelisaurus gobiensis (IVPP V9492). Values are given in millimetres. Underlined values indicate the element is incompletely preserved and italicized values indicate approximations. Abbreviations: FL, functional length; PCH, posterior centrum height; PCW, posterior centrum width.

\begin{tabular}{|r|r|c|c|}
\hline & FL & PCH & PCW \\
\hline '18' & 133 & 112 & 100 \\
\hline '19' & 147 & 99 & 94 \\
\hline '20' & 156 & - & - \\
\hline '21' & $\underline{135}$ & 99 & - \\
\hline '22' & 139 & 91 & 79 \\
\hline '23' & 116 & 101 & 77 \\
\hline '24' & 132 & 106 & 67 \\
\hline '25' & 135 & 78 & 69 \\
\hline '26' & 139 & $\underline{80}$ & $\underline{66}$ \\
\hline '27' & 76 & - & - \\
\hline '33' & 81 & 56 & 51 \\
\hline
\end{tabular}


Table 6. Dimensions of the chevrons of Klamelisaurus gobiensis (IVPP V9492). Values are given in millimetres. Italicized values indicate approximations. Abbreviations: HCH, height of haemal canal; HCW, maximum width of haemal canal; PDH, maximum proximodistal height along the midline; PL, proximal anteroposterior length; PW, proximal transverse width.

\begin{tabular}{|c|c|c|c|c|c|}
\hline Chevron & PDH & PW & PL & HCH & HCW \\
\hline '26' & 102 & 68 & 30 & 47 & 15 \\
\hline '28' & 90 & 65 & 30 & 33 & 10 \\
\hline '29' & 81 & 67 & 21 & 30 & 19 \\
\hline '30' & 72 & 56 & 29 & 35 & 11 \\
\hline
\end{tabular}


Table 7. Measurements of the right scapula and coracoid of Klamelisaurus gobiensis (IVPP V9492). Values are given in millimetres or degrees, as appropriate. Italicized values are approximations.

\begin{tabular}{|c|c|c|}
\hline Element & Dimension & Measurements \\
\hline \multirow[t]{6}{*}{ Scapula } & Minimum breadth of blade proximally & 158 \\
\hline & $\begin{array}{l}\text { Acromion dorsoventral height, perpendicular to the } \\
\text { blade, measured between horizontal lines extending } \\
\text { through dorsal- and ventral-most points of acromion }\end{array}$ & 540 \\
\hline & Angle of blade with respect to coracoid articulation & 83 \\
\hline & $\begin{array}{l}\text { Anteroposterior breadth of scapular blade, from } \\
\text { posterior margin to anterodorsal corner, where it } \\
\text { meets the coracoid }\end{array}$ & 380 \\
\hline & Length of glenoid & 188 \\
\hline & Maximum width of glenoid & 142 \\
\hline \multirow[t]{4}{*}{ Coracoid } & Anteroposterior length & 360 \\
\hline & Maximum dorsoventral height & 370 \\
\hline & Length of glenoid & 130 \\
\hline & Maximum width of glenoid & 123 \\
\hline Glenoid & Greatest expanse of glenoid & 267 \\
\hline
\end{tabular}


Table 8. Measurements of the right forelimb of Klamelisaurus gobiensis (IVPP V9492). Values given in millimetres. Italicized values indicate approximations.

\begin{tabular}{|c|c|c|}
\hline Element & Dimension & Measurement \\
\hline \multirow[t]{9}{*}{ Humerus } & Proximodistal length & 880 \\
\hline & Transverse width of proximal end & 363 \\
\hline & Anteroposterior width across humeral head & 118 \\
\hline & Midshaft transverse width & 148 \\
\hline & Minimum midshaft circumference & 422 \\
\hline & Midshaft anteroposterior width & 95 \\
\hline & Transverse width of distal end & 310 \\
\hline & $\begin{array}{c}\text { Maximum anteroposterior width of distal end (across } \\
\text { ulnar condyle) }\end{array}$ & 161 \\
\hline & $\begin{array}{c}\text { Distance from proximal end to most prominent point of } \\
\text { deltopectoral crest }\end{array}$ & 340 \\
\hline \multirow[t]{10}{*}{ Ulna } & Proximodistal length & 605 \\
\hline & $\begin{array}{l}\text { Length of anteromedial process } \\
\text { (following Upchurch et al. 2015) }\end{array}$ & 154 \\
\hline & Maximum length along anteromedial process & 218 \\
\hline & $\begin{array}{l}\text { Length of anterolateral process } \\
\text { (following Upchurch et al. 2015) }\end{array}$ & 105 \\
\hline & Maximum length along anterolateral process & 192 \\
\hline & Midshaft transverse width & 92 \\
\hline & Midshaft anteroposterior width & 130 \\
\hline & Midshaft circumference & 326 \\
\hline & Transverse width of distal end & 103 \\
\hline & Anteroposterior width of distal end & 134 \\
\hline \multirow[t]{5}{*}{ Radius } & Proximodistal length & 540 \\
\hline & Transverse width of proximal end & 146 \\
\hline & Anteroposterior width of proximal end & 108 \\
\hline & Midshaft transverse width & 86 \\
\hline & Midshaft anteroposterior width & 67 \\
\hline
\end{tabular}




\begin{tabular}{|c|c|c|}
\hline Element & Dimension & Measurement \\
\hline & Midshaft circumference & 245 \\
\hline & Transverse width of distal end & 117 \\
\hline & Anteroposterior width of distal end & 115 \\
\hline
\end{tabular}


Table 9. Measurements of the right ilium of Klamelisaurus gobiensis (IVPP V9492). Values given in millimetres. Underlined values indicate the element is incompletely preserved and italicized values indicate approximations.

\begin{tabular}{|c|c|}
\hline Dimension & Measurement \\
\hline Length of ilium along longest axis & $\underline{690}$ \\
\hline Length of pubic peduncle & $\underline{137}$ \\
\hline Anteroposterior length of distal end of pubic peduncle & $\underline{80}$ \\
\hline Transverse width of distal end of pubic peduncle & $\underline{148}$ \\
\hline Anteroposterior length of distal end of ischial peduncle & $\underline{116}$ \\
\hline Transverse width of distal end of ischial peduncle & $\underline{136}$ \\
\hline Greatest transverse width of acetabulum & 167 \\
\hline
\end{tabular}


Table 10. Measurements of the hind limb of Klamelisaurus gobiensis (IVPP V9492). Values given in millimetres. Italicized values indicate approximations. Asterisk indicates values given by Zhao (1993) that could not be reproduced owing to poor preservation or reconstruction. Abbreviations: L, left; R, right.

\begin{tabular}{|c|c|c|}
\hline Element & Dimension & Measurement \\
\hline \multirow[t]{8}{*}{ Femur (L) } & Distal transverse width & 323 \\
\hline & Anteroposterior width of tibial condyle & 251 \\
\hline & Anteroposterior width of fibular condyle & 203 \\
\hline & Midshaft transverse width & 189 \\
\hline & Midshaft anteroposterior width & 115 \\
\hline & Minimum shaft circumference & 525 \\
\hline & Transverse width of tibial condyle & 93 \\
\hline & Transverse width of fibular condyle & 117 \\
\hline \multirow[t]{7}{*}{ Femur (R) } & Proximodistal length & 1240 \\
\hline & Proximal transverse width & 325 \\
\hline & Proximal anteroposterior width & 135 \\
\hline & $\begin{array}{l}\text { Distance from proximal end to maximum height of } \\
\text { fourth trochanter }\end{array}$ & 530 \\
\hline & Minimum shaft circumference & 556 \\
\hline & Midshaft transverse width & 198 \\
\hline & Midshaft anteroposterior width & 132 \\
\hline \multirow[t]{6}{*}{ Tibia (L) } & Proximodistal length & 740 \\
\hline & Maximum breadth of proximal end & 216 \\
\hline & Width orthogonal to maximum proximal breadth & 170 \\
\hline & Midshaft transverse width & 145 \\
\hline & Midshaft anteroposterior width & 67 \\
\hline & Midshaft circumference & 386 \\
\hline \multirow[t]{3}{*}{ Tibia (R) } & Proximodistal length & $740^{\star}$ \\
\hline & Proximal breadth & $240^{*}$ \\
\hline & Distal breadth & $190^{*}$ \\
\hline Fibula (L) & Proximodistal length & 750 \\
\hline
\end{tabular}




\begin{tabular}{|c|r|c|}
\hline Element & Dimension & Measurement \\
\hline & Maximum breadth of proximal end & 178 \\
\hline & Width orthogonal to maximum proximal breadth & 90 \\
\hline Midshaft transverse width & 71 \\
\hline & Midshaft anteroposterior width & 111 \\
\hline & Proximodistal length & $770^{*}$ \\
\hline & Proximal breadth & $190^{\star}$ \\
\hline
\end{tabular}


Table 11. Measurements of the pedal elements of Klamelisaurus gobiensis (IVPP V9492). Values are given in millimetres. Asterisk indicates the position of the element as mounted; we interpret these elements as being pedal phalanges. Italicized values indicate approximations. Abbreviations: L, left; R, right.

\begin{tabular}{|c|c|c|c|c|c|c|c|c|}
\hline Element & Side & Length & $\begin{array}{l}\text { Proximal } \\
\text { width }\end{array}$ & $\begin{array}{c}\text { Proximal } \\
\text { height }\end{array}$ & $\begin{array}{l}\text { Mid } \\
\text { width }\end{array}$ & $\begin{array}{c}\text { Mid } \\
\text { height }\end{array}$ & $\begin{array}{l}\text { Distal } \\
\text { width }\end{array}$ & $\begin{array}{l}\text { Distal } \\
\text { height }\end{array}$ \\
\hline Metatarsal I & ?R & 130 & 72 & 98 & 63 & 59 & 85 & 49 \\
\hline $\begin{array}{c}\text { Metatarsal II } \\
\text { or III }\end{array}$ & ?R & 135 & 80 & - & - & - & 65 & - \\
\hline $\begin{array}{c}\text { Pedal } \\
\text { phalanx III-1 }\end{array}$ & $\mathbf{L}$ & 63 & 102 & 79 & 98 & - & 101 & 75 \\
\hline $\begin{array}{l}\text { Pedal } \\
\text { phalanx } \\
\text { III-1* }^{\star}\end{array}$ & $\mathbf{R}$ & 66 & 108 & - & 96 & - & 105 & 71 \\
\hline $\begin{array}{c}\text { Pedal } \\
\text { phalanx } \\
\text { IV-3* }^{\star}\end{array}$ & $\mathbf{L}$ & 50 & 70 & 58 & 64 & 49 & 60 & 36 \\
\hline $\begin{array}{c}\text { Pedal } \\
\text { phalanx }\end{array}$ & $\mathbf{R}$ & 160 & 55 & 97 & - & - & - & - \\
\hline
\end{tabular}



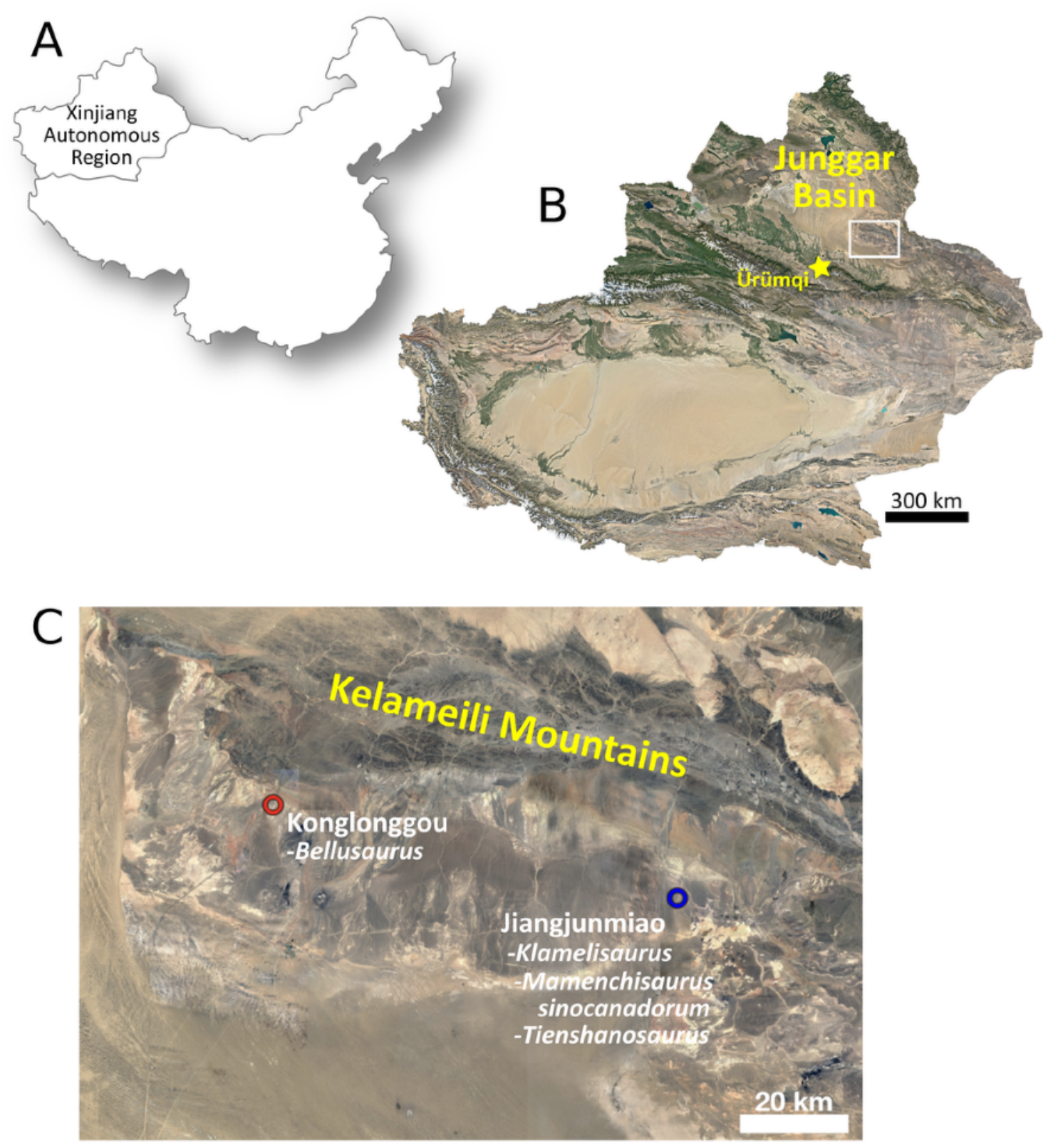

Figure 1. Occurrence of Klamelisaurus gobiensis (IVPP V9492). A, map of China, demarcating the Xinjiang Autonomous Region; B, close-up of Xinjiang Autonomous Region, highlighting the location of Shishugou Formation outcrops; C, collection sites of sauropod dinosaurs from the Shishugou Formation. Map credit: Google Earth, US Dept of State Geographer, Image Landsat / Copernicus, (C) 2017 Google. 


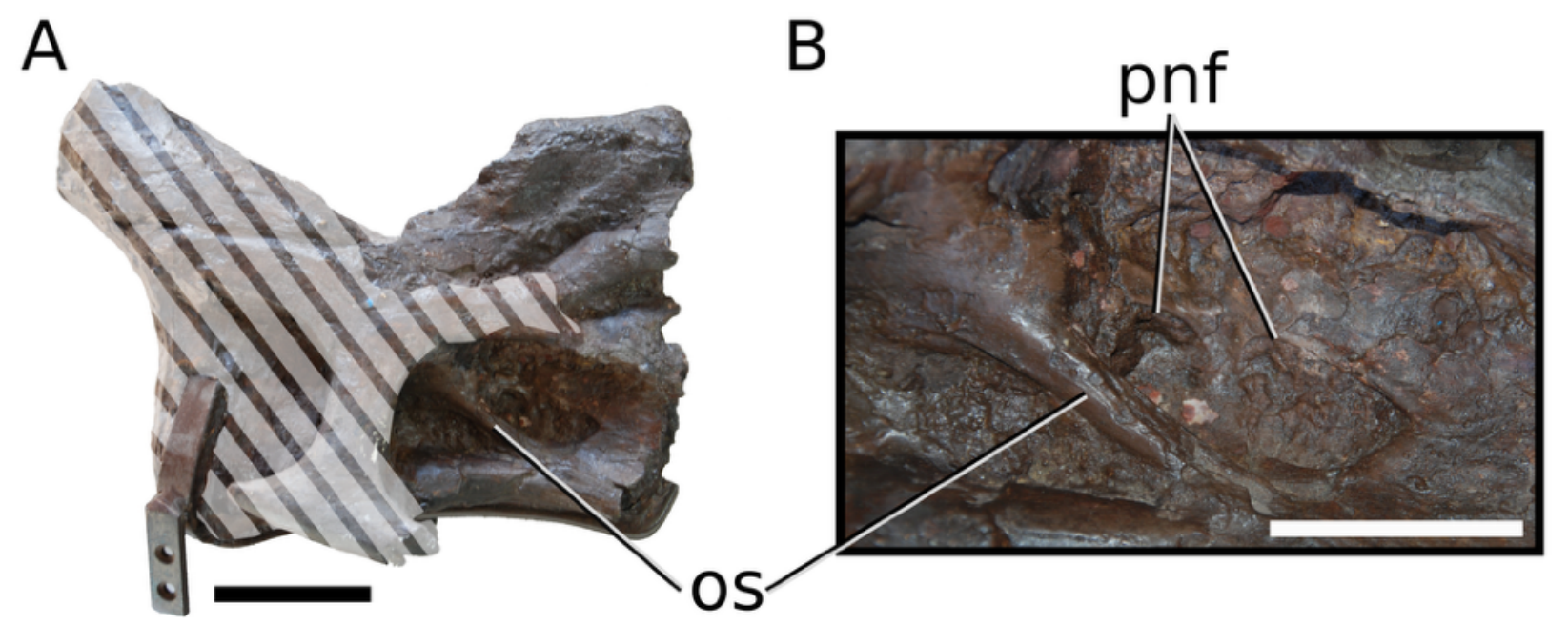

Figure 2. Cervical vertebrae 8 and 9 of Klamelisaurus gobiensis (IVPP V9492). Only part of the posterior half of cervical 8 and the anterior half of cervical 9 are present in this block; the remainder of cervical 9 is preserved in articulation with cervical 10. A, left lateral view; B, closeup of left lateral pneumatic fossa of cervical 9, highlighting the anterodorsally oblique strut and adjacent, sharp-lipped pneumatic foramina. Abbreviations: os, anterodorsally oblique strut; pnf, pneumatic foramen. Striped regions indicate reconstruction. Scale bars: $A=10 \mathrm{~cm} ; \mathrm{B}=5 \mathrm{~cm}$. 


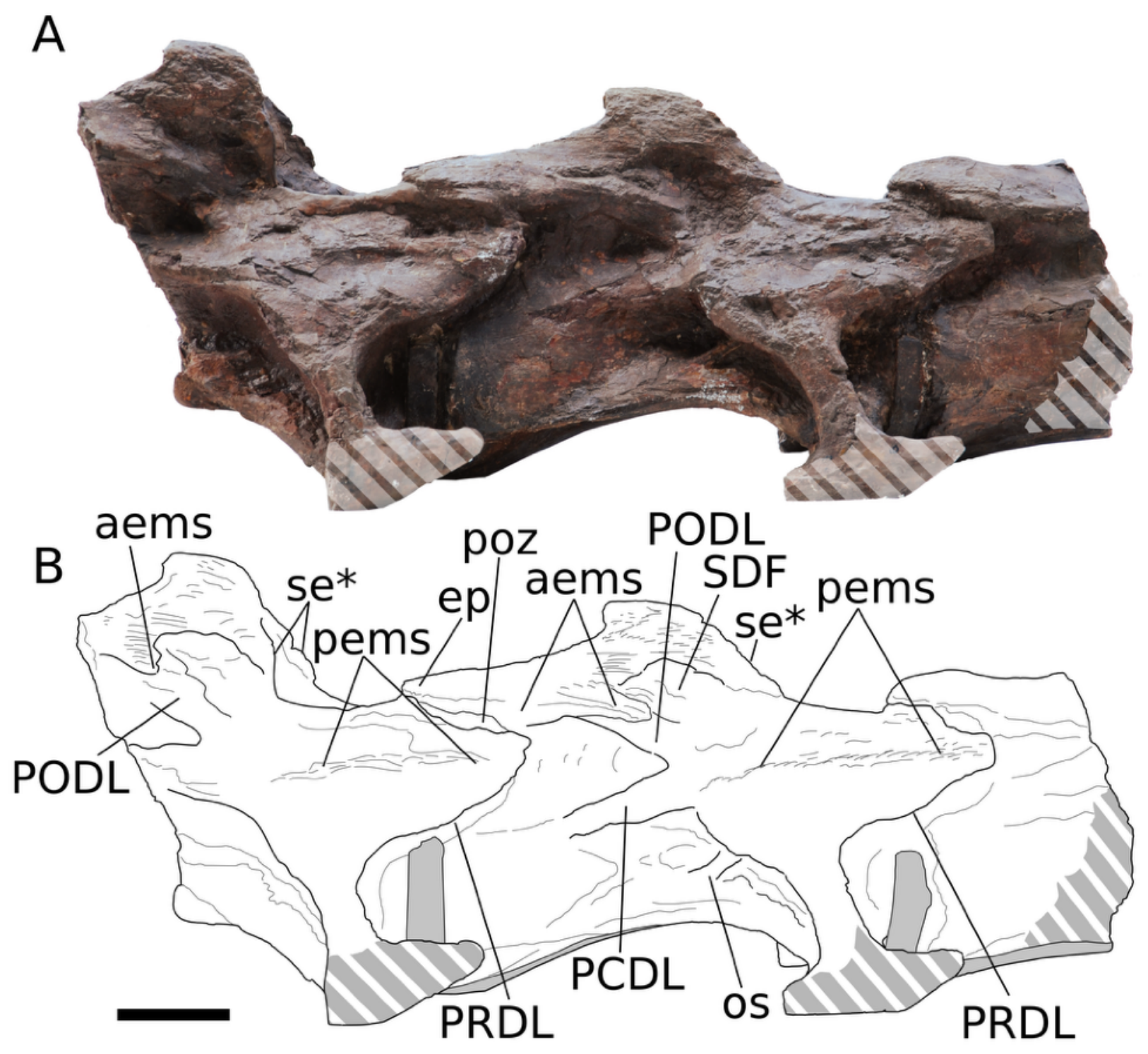

Figure 3. Cervical vertebrae 9-11 of Klamelisaurus gobiensis (IVPP V9492). A, right lateral view; B, schematic of right lateral view. Note the gentle convexity of the prezygodiapophyseal lamina in cervicals 10 and 11. Abbreviations: aems, anterior epipophyseal muscle scar; ep, epipophysis; os, anterodorsally oblique strut; PCDL, posterior centrodiapophyseal lamina; pems, pre-epipophyseal muscle scar; PODL, postzygodiapophyseal lamina; poz, postzygapophysis; PRDL, prezygodiapophyseal lamina; SDF, spinodiapophyseal fossa; se, sheet-like extensions of the SPRL. Asterisk indicates an autapomorphy. Striped regions indicate reconstruction. Scale bar $=10 \mathrm{~cm}$. 

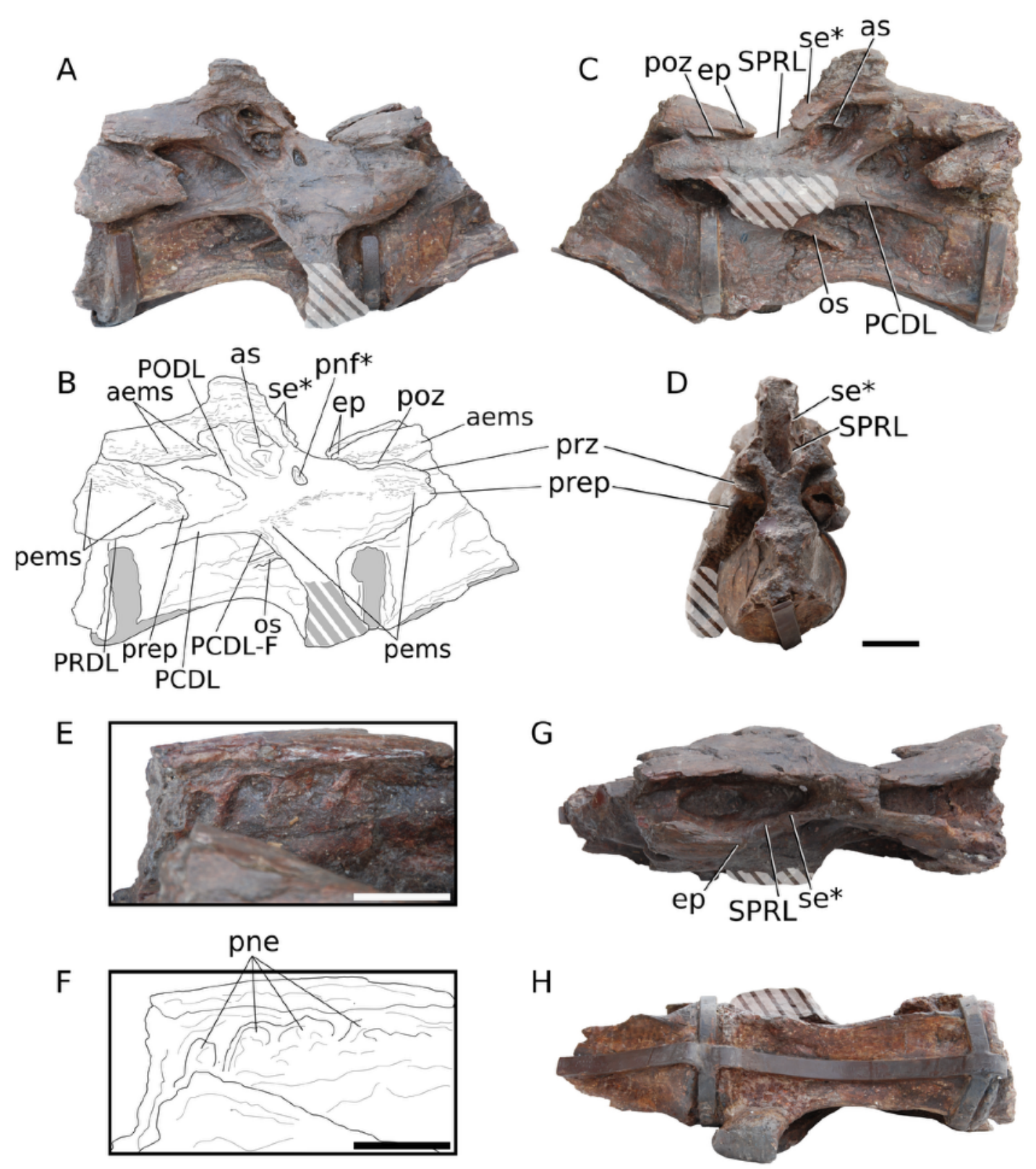

Figure 4. Cervical vertebrae 11-13 of Klamelisaurus gobiensis (IVPP V9492). A, right lateral view; B, schematic of right lateral view. Note the convexity of the prezygodiapophyseal lamina in right lateral view; $\mathrm{C}$, left lateral view; $\mathrm{D}$, anterior view; $\mathrm{E}$, internal surface of the right spinopostzygapophyseal lamina of cervical $11 ; \mathrm{F}$, schematic of internal surface of right spinopostzygapophyseal lamina of cervical 11. Note the sharp-lipped pneumatic embayments; G, dorsal view; H, ventral view. Abbreviations: aems, anterior epipophyseal muscle scar; as, accessory strut; ep, epipophysis; os, anterodorsally oblique strut; PCDL, posterior centrodiapophyseal lamina; PCDL-F, posterior centrodiapophyseal lamina fossa; pems, preepipophyseal muscle scar; pne, pneumatic excavation; pnf, pneumatic foramen; PODL, postzygodiapophyseal lamina; poz, postzygapophysis; PRDL, prezygodiapophyseal lamina; 
prep, pre-epipophysis; prz, prezygapophysis; se, sheet-like extensions of the spinoprezygapophyseal lamina; SPRL, spinoprezygapophyseal lamina. Asterisk indicates an autapomorphy. Striped regions indicate reconstruction. Scale bars: A-D, G, H $=10 \mathrm{~cm} ; \mathrm{E}, \mathrm{F}=5$ $\mathrm{cm}$. 


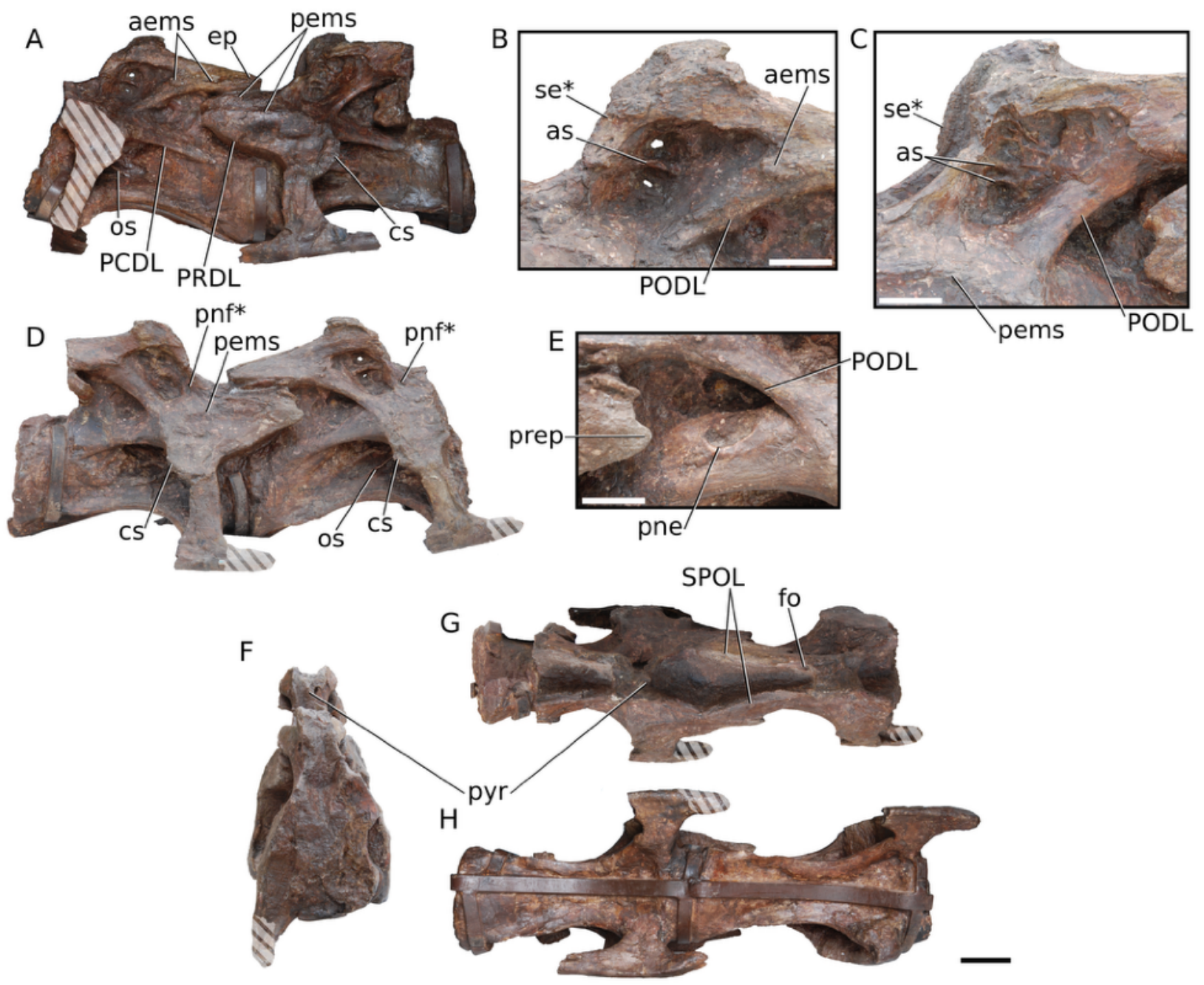

Figure 5. Cervical vertebrae 13 and 14 of Klamelisaurus gobiensis (IVPP V9492). A, left lateral view; B, close-up of left spinodiapophyseal fossa of cervical 13; C, close-up of left spinodiapophyseal fossa of cervical 14; D, right lateral view; E, close-up of right postzygapophyseal centrodiapophyseal fossa of cervical 13; F, anterior view; G, dorsal view; H, ventral view. Abbreviations: aems, anterior epipophyseal muscle scar; as, accessory strut; cs, costal spur; ep, epipophysis; fo, fossa; os, anterodorsally oblique strut; PCDL, posterior centrodiapophyseal lamina; pems, pre-epipophyseal muscle scar; pne, pneumatic excavation; pnf, pneumatic foramen; PODL, postzygodiapophyseal lamina; PRDL, prezygodiapophyseal lamina; prep, pre-epipophysis; pyr, pyramidal boss; se, sheet-like extensions of the SPRL; SPOL, spinopostzygapophyseal lamina. Asterisk indicates an autapomorphy. Striped regions indicate reconstruction. Scale bars: A, F-H $=10 \mathrm{~cm} ; \mathrm{B}, \mathrm{C}, \mathrm{E}=5 \mathrm{~cm}$. 


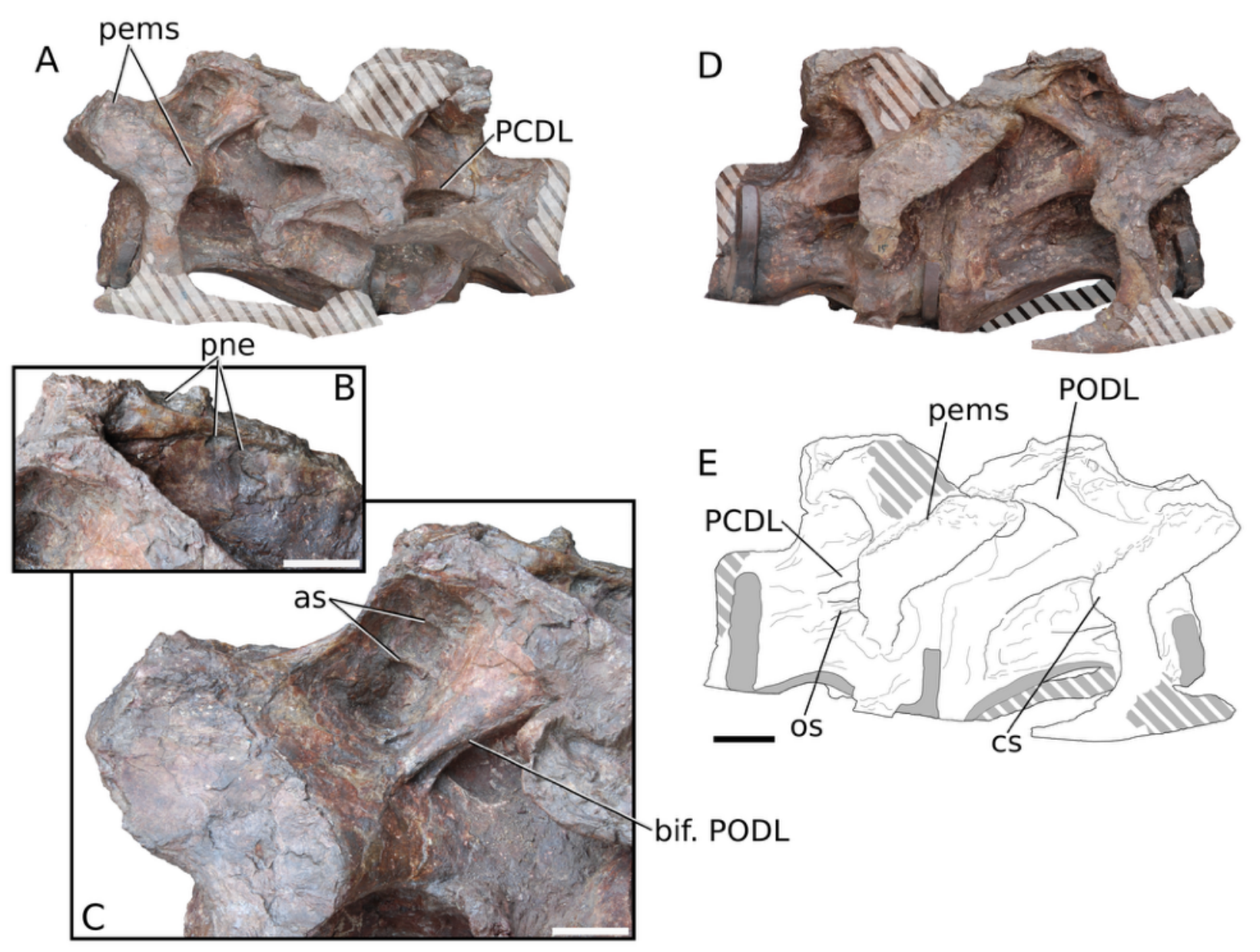

Figure 6. Cervical vertebrae 15 and 16 of Klamelisaurus gobiensis (IVPP V9492). A, left lateral view; B, close-up, left dorsolateral view of the neural spine and spinopostzygapophyseal laminae of cervical 15; C, close-up, left posterolateral view of neural arch of cervical 15. Note the ventrally bifurcated postzygodiapophyseal lamina; D, right lateral view; E, schematic of right lateral view. Abbreviations: as, accessory strut; bif. PODL, bifurcated postzygodiapophyseal lamina; cs, costal spur; os, anterodorsally oblique lamina; PCDL, posterior centrodiapophyseal lamina; pems, pre-epipophyseal muscle scar; pne, pneumatic excavation; PODL, postzygodiapophyseal lamina. Striped regions indicate reconstruction. Scale bars: A, D, E = 10 $\mathrm{cm} ; \mathrm{B}, \mathrm{C}=5 \mathrm{~cm}$. 

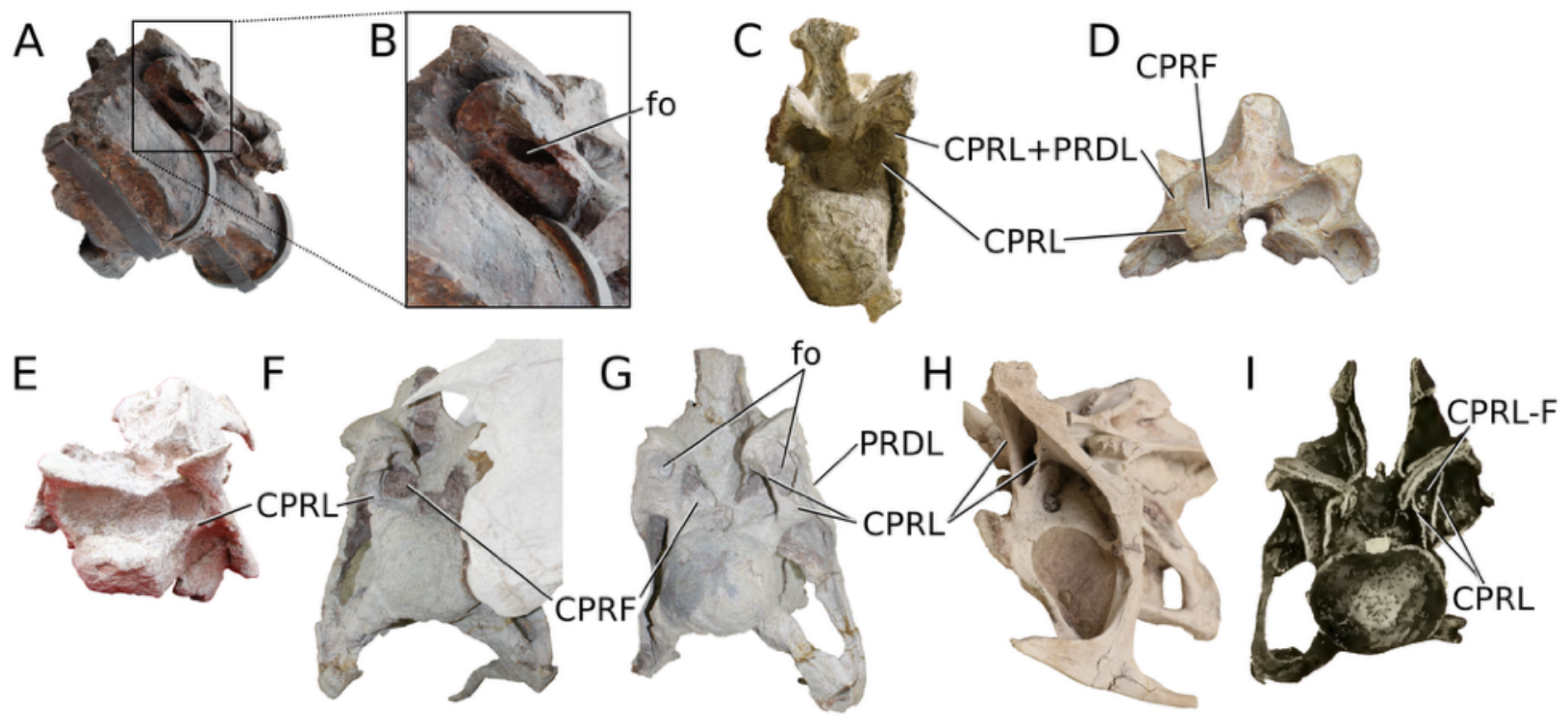

Figure 7. Morphology of the centroprezygapophyseal lamina in Klamelisaurus gobiensis and other sauropods. A, left anteroventrolateral view of cervical 12 of Klamelisaurus gobiensis; B, close-up of cervical 12; C, anterior view of cervical 11 of Jobaria (MNBH TIG 6); D, anterior view of an anterior cervical neural arch of Bellusaurus (IVPP V17768 03-53); E, anterior view of cervical 3 of Qijianglong (QJGPM 1001); F, anterior view of cervical 9 of Qijianglong (QJGPM 1001); G, anterior view of cervical 14 of Qijianglong (QJGPM 1001); H, left anterolateral view of cervical 6 of Nigersaurus (MNBH GAD 512); I, anterior view of cervical 14 of Diplodocus carnegii (CM 84), modified from Hatcher (1901, pl. V). Note the presence of lateral and medial centroprezygapophyseal laminae that both contact the prezygapophysis dorsally. See also Tschopp et al. (2015a, fig. 54). Abbreviations: CPRF, centroprezygapophyseal fossa; CPRL, centroprezygapophyseal lamina; CPRL-F, centroprezygapophyseal lamina fossa; CPRL+PRDL, conjoined centroprezygapophyseal lamina and prezygodiapophyseal lamina; fo, fossa. Images of Qijianglong used with permission of T. Miyashita. Not to scale. 

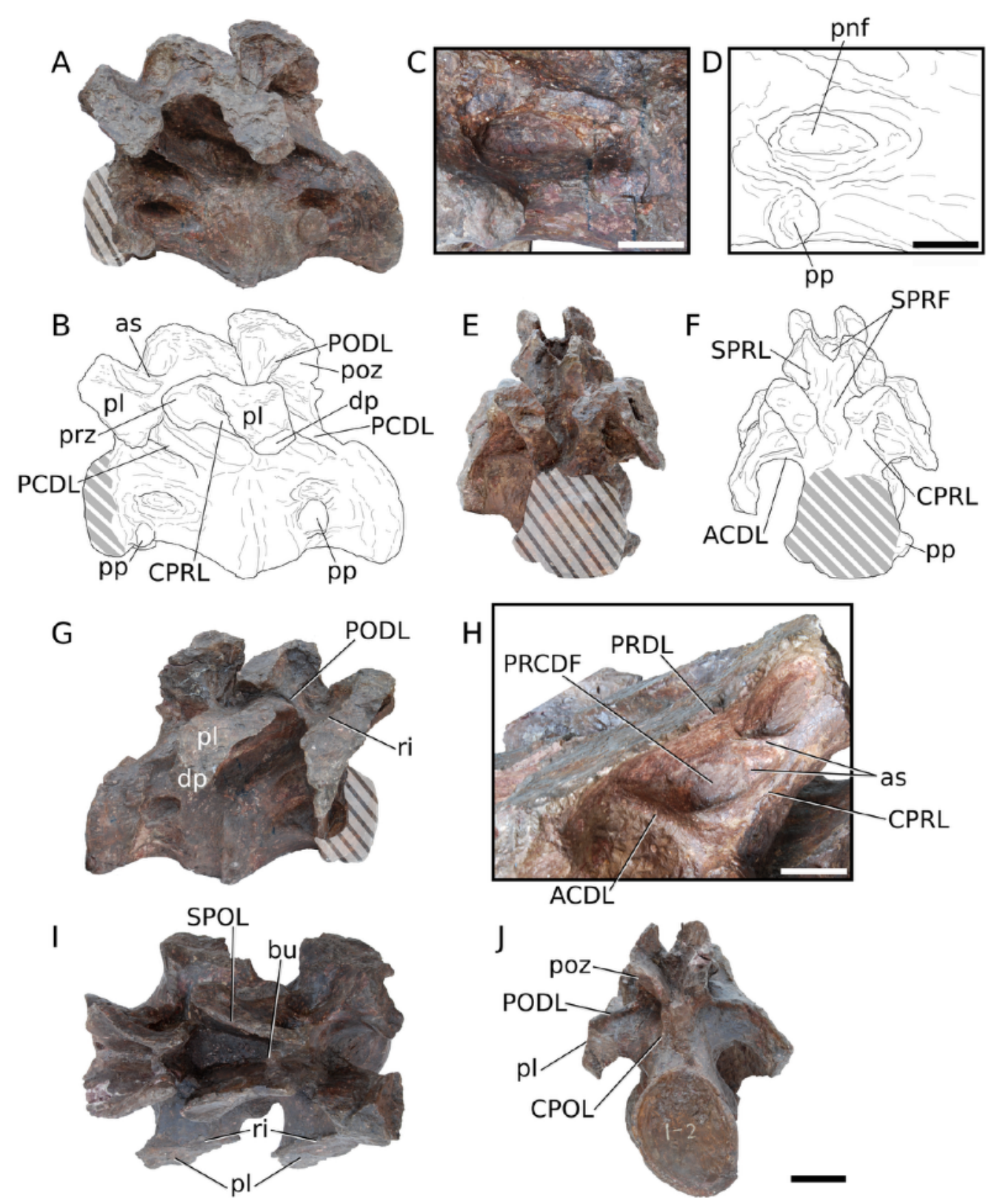

Figure 8. Dorsal vertebrae 1 and 2 of Klamelisaurus gobiensis (IVPP V9492). A, left lateral view; B, schematic of left lateral view; C, close-up view of left lateral pneumatic excavation of the centrum of dorsal 1 . Note the acute posterior margin of the pneumatic foramen, which is set within a larger fossa; D, schematic of left lateral pneumatic excavation of the centrum of dorsal $1 ; \mathrm{E}$, anterior view; F, schematic of anterior view, omitting in-place matrix supporting the right diapophysis; $\mathrm{G}$, right lateral view; $\mathrm{H}$, close-up view of right prezygapophyseal centrodiapophyseal fossa. Note the accessory struts dividing the fossa; I, dorsal view; J, posterior view. Abbreviations: ACDL, anterior centrodiapophyseal lamina; as, accessory strut; bu, bump; CPOL, centropostzygapophyseal lamina; CPRL, centroprezygapophyseal lamina; dp, diapophysis; PCDL, posterior centrodiapophyseal lamina; pl, dorsolaterally facing platform of the diapophysis; pnf, pneumatic foramen; PODL, postzygodiapophyseal lamina; poz, postzygapophysis; pp, parapophysis; PRCDF, prezygapophyseal centrodiapophyseal fossa; PRDL, prezygodiapophyseal lamina; ri, ridge; SPRF, spinoprezygapophyseal fossa; SPRL, 
spinoprezygapophyseal lamina. Striped regions indicate reconstruction. Scale bars: A, B, E-G, I, $\mathrm{J}=10 \mathrm{~cm} ; \mathrm{C}, \mathrm{D}, \mathrm{H}=5 \mathrm{~cm}$. 

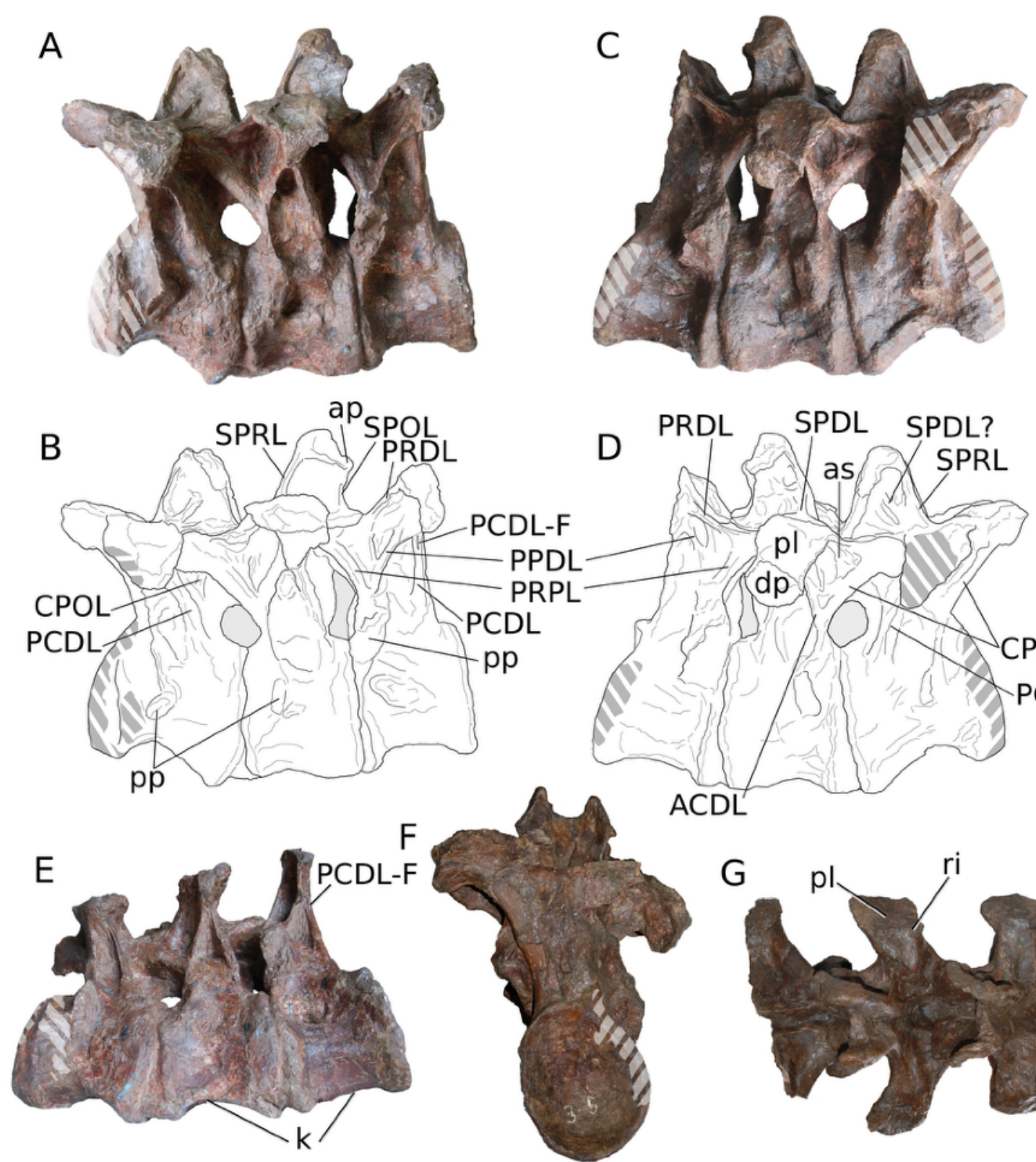

PRDL
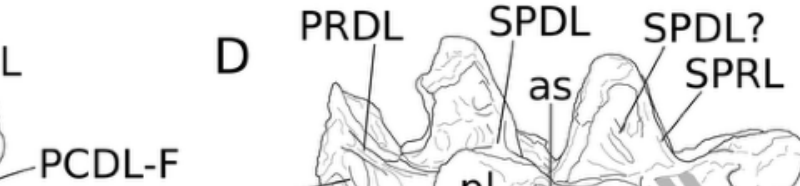
PPDL PRPL PCDL pp

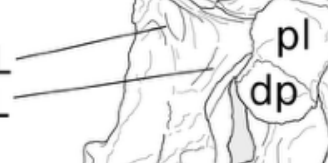




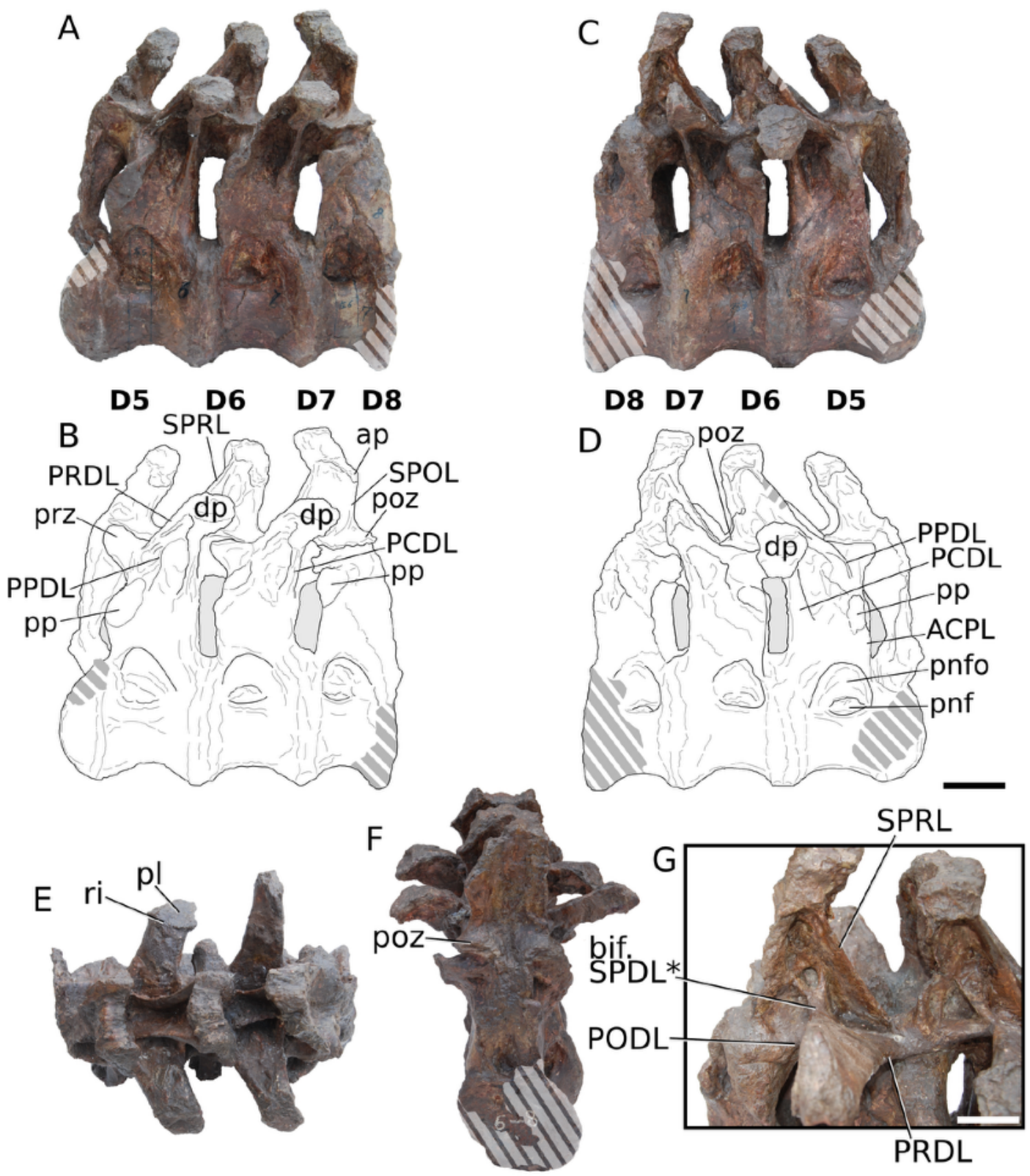

Figure 10. Dorsal vertebrae 5-8 of Klamelisaurus gobiensis (IVPP V9492). A, left lateral view; $\mathrm{B}$, schematic of left lateral view; C, right lateral view; D, schematic of right lateral view; E, dorsal view; F, posterior view; G, close-up of right dorsolateral view of spinodiapophyseal fossae of dorsals 6 and 7. Note bifurcated spinodiapophyseal lamina and subfossae within the spinodiapophyseal fossa. Abbreviations: ACPL, anterior centroparapophyseal lamina; ap, aliform process; bif. SPDL, bifurcated spinodiapophyseal lamina; D, dorsal; dp, diapophysis; PCDL, posterior centrodiapophyseal lamina; pl, dorsolaterally facing platform of the diapophysis; pnf, pneumatic foramen; pnfo, pneumatic fossa; PODL, postzygodiapophyseal lamina; poz, postzygapophysis; pp, parapophysis; PPDL, paradiapophyseal lamina; PRDL, prezygodiapophyseal lamina; prz, prezygapophysis; ri, ridge; SPOL, spinopostzygapophyseal lamina; SPRL, spinoprezygapophyseal lamina. Asterisk indicates an autapomorphy. Striped regions indicate reconstruction. Scale bars: $A-F=10 \mathrm{~cm} ; G=5 \mathrm{~cm}$. 
A

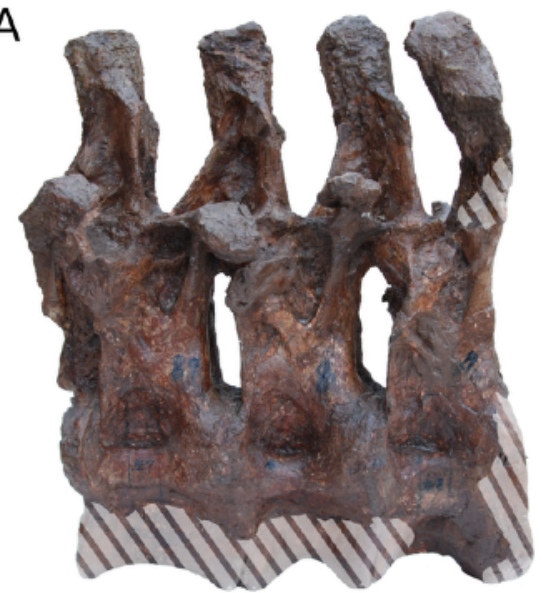

D8 D9 D10 D11
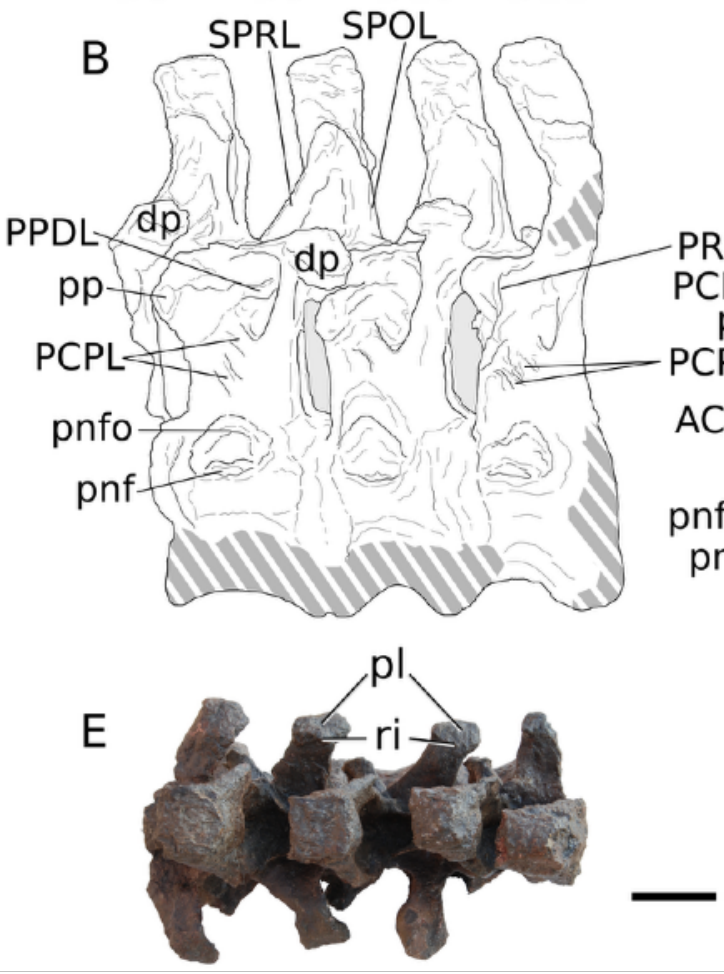

C

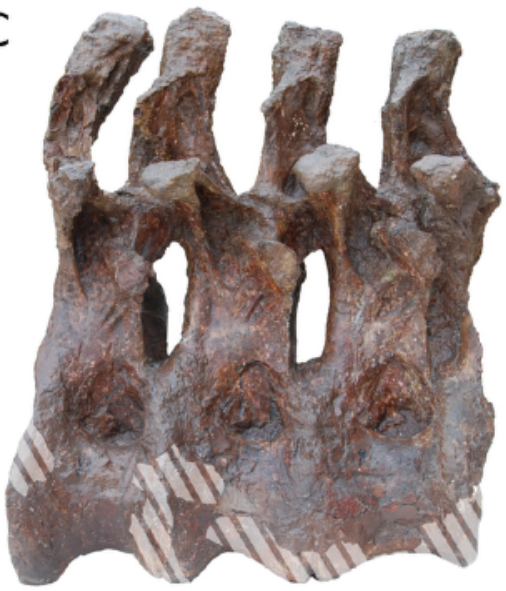

D11 D10 D9 D8

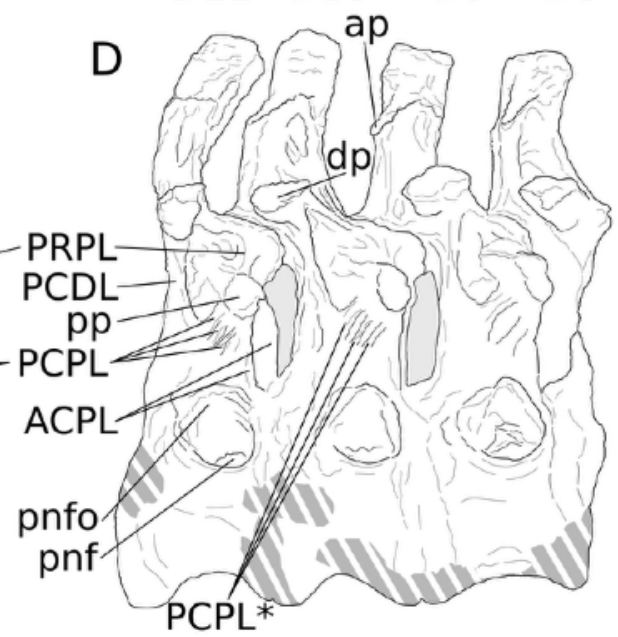

Figure 11. Dorsal vertebrae 8-11 of Klamelisaurus gobiensis (IVPP V9492). A, left lateral view; $\mathrm{B}$, schematic of left lateral view; C, right lateral view; D, schematic of right lateral view; E, dorsal view. Abbreviations: ap, aliform process; D, dorsal; dp, diapophysis; PCDL, posterior centrodiapophyseal lamina; PCPL, posterior centroparapophyseal lamina; pnf, pneumatic foramen; pnfo, pneumatic fossa; $\mathrm{pl}$, dorsolaterally facing platform of the diapophysis; $\mathrm{pp}$, parapophysis; PPDL, paradiapophyseal lamina; PRPL, prezygoparapophyseal lamina; ri, ridge; SPOL, spinopostzygapophyseal lamina; SPRL, spinoprezygapophyseal lamina. Asterisk indicates an autapomorphy. Striped regions indicate reconstruction. Scale bar $=10 \mathrm{~cm}$. 


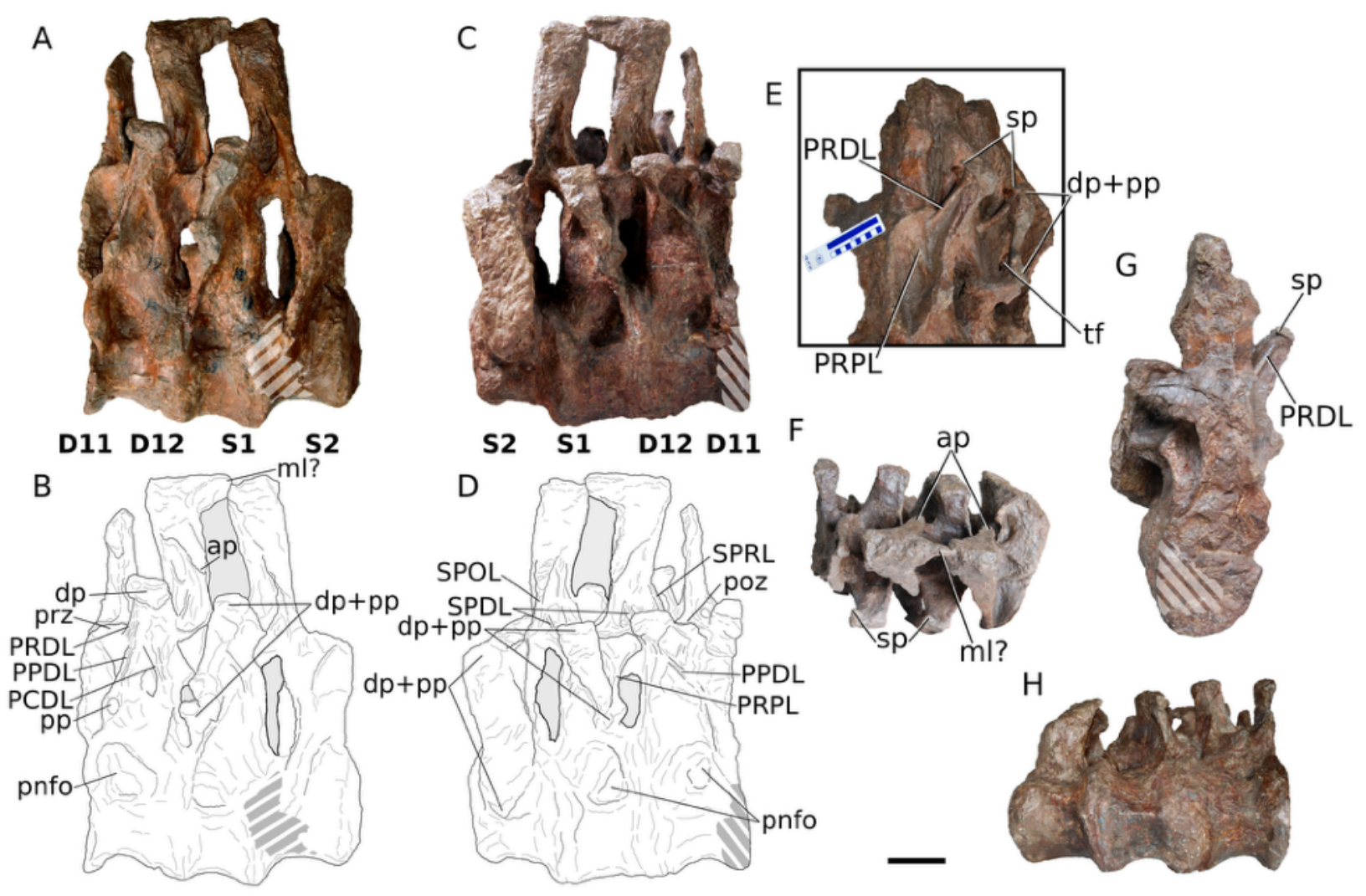

Figure 12. Dorsal vertebrae 11 and 12 and sacral vertebrae 1 and 2 of Klamelisaurus gobiensis (IVPP V9492). A, left lateral view; B, schematic of left lateral view; C, right lateral view; D, schematic of right lateral view; E, left anterolateral view. Note transverse foramen bounded by diapophysis, parapophysis and a bridge of bone connecting these landmarks; F, dorsal view; G, anterior view; $\mathrm{H}$, right ventrolateral view. Abbreviations: ap, aliform process; $\mathrm{D}$, dorsal; dp, diapophysis; dp1pp, conjoined diapophysis and parapophysis; ml, mineralized ligament; PCDL, posterior centrodiapophyseal lamina; pnfo, pneumatic fossa; pp, parapophysis; PPDL, paradiapophyseal lamina; PRDL, prezygodiapophyseal lamina; PRPL, prezygoparapophyseal lamina; prz, prezygapophysis; S, sacral; sp, spur; SPDL, spinodiapophyseal lamina; SPOL, spinopostzygapophyseal lamina; SPRL, spinoprezygapophyseal lamina; tf, transverse foramen. Striped regions indicate reconstruction. Scale bar $=10 \mathrm{~cm}$. 


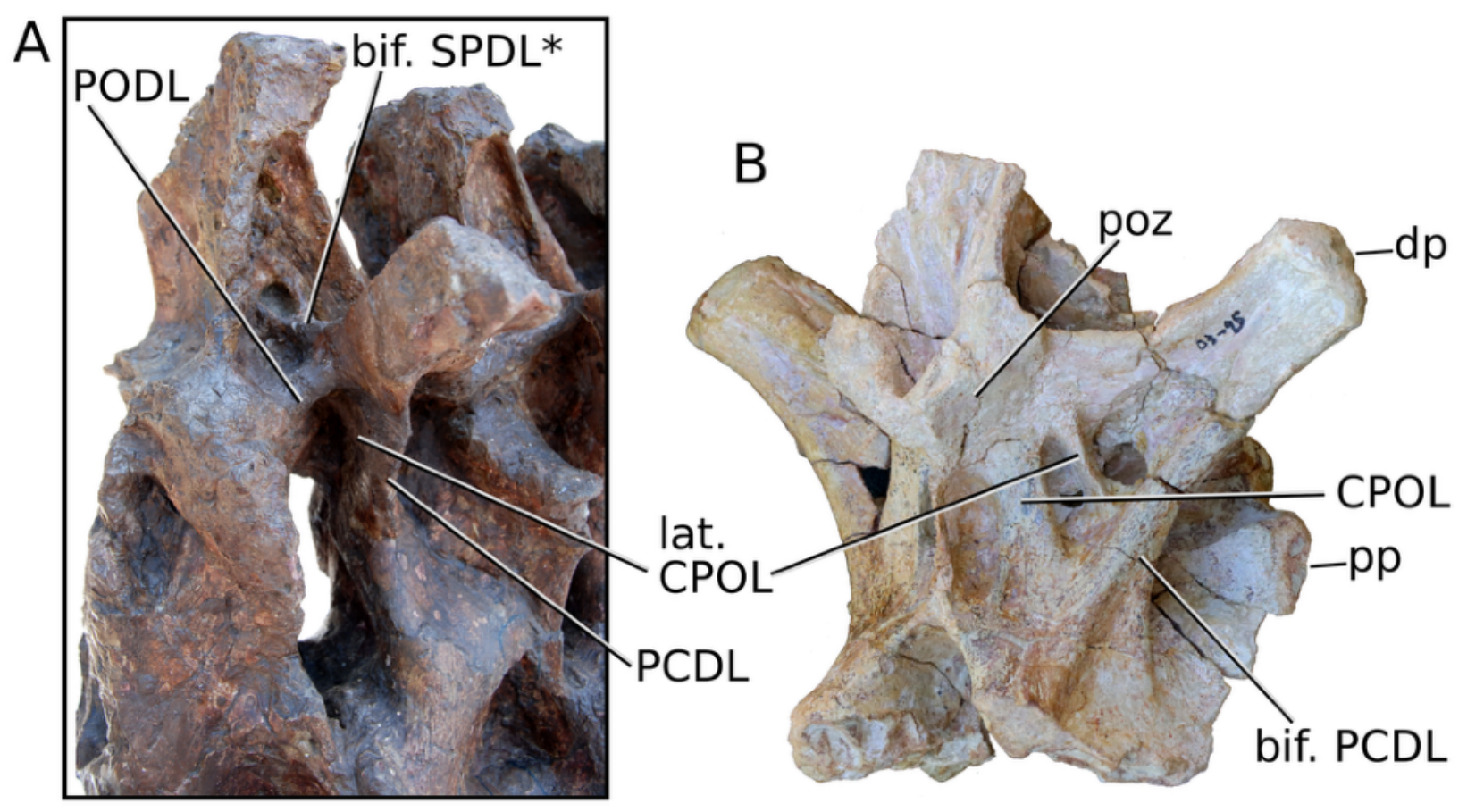

Figure 13. Lateral centropostzygapophyseal lamina in posterior dorsal vertebrae of Klamelisaurus gobiensis and Bellusaurus sui. A, right posterolateral view of dorsal 7 of Klamelisaurus gobiensis (IVPP V9492); B, right posterolateral view of a posterior dorsal neural arch of Bellusaurus sui (IVPP V17768 03-95). Note the well-defined fossa in which the lateral centropostzygapophyseal lamina is placed in Bellusaurus; no such fossa is present in Klamelisaurus. IVPP V17768 03-95 also differs from middle-posterior dorsal vertebrae of Klamelisaurus in several other respects, including having a ventrally bifurcate posterior centrodiapophyseal lamina, lacking a posterior centroparapophyseal lamina, having strongly dorsolaterally directed diapophyses, and having steeply inclined zygapophyseal facets. Abbreviations: bif. PCDL, ventrally bifurcated posterior centrodiapophyseal lamina; bif. SPDL, bifurcated spinodiapophyseal lamina; CPOL, centropostzygapophyseal lamina; dp, diapophysis; lat. CPOL, lateral centropostzygapophyseal lamina; PCDL, posterior centrodiapophyseal lamina; PODL, postzygodiapophyseal lamina; poz, postzygapophysis; pp, parapophysis. Asterisk indicates autapomorphy. Not to scale. 


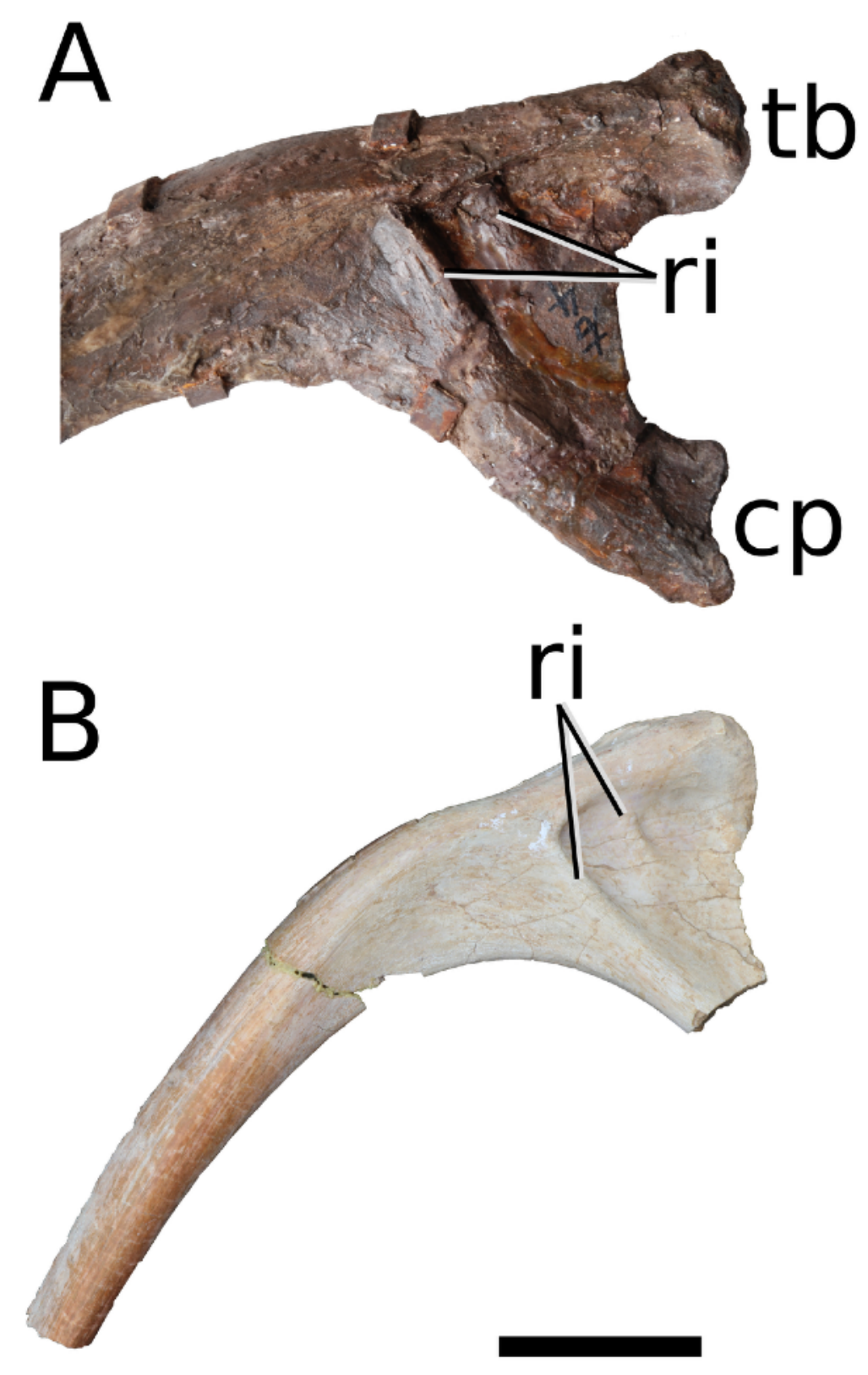

Figure 14. Dorsal ribs of Klamelisaurus gobiensis and Bellusaurus sui. A, dorsal rib ?4 of Klamelisaurus gobiensis (IVPP V9492) in anterior view; B, anterior dorsal rib of Bellusaurus sui (IVPP V17768.212) in anterior view. Abbreviations: cp, capitulum; ri, ridge; tb, tuberculum. Scale bar: $\mathrm{A}=10 \mathrm{~cm} ; \mathrm{B}=5 \mathrm{~cm}$. 


\section{A}

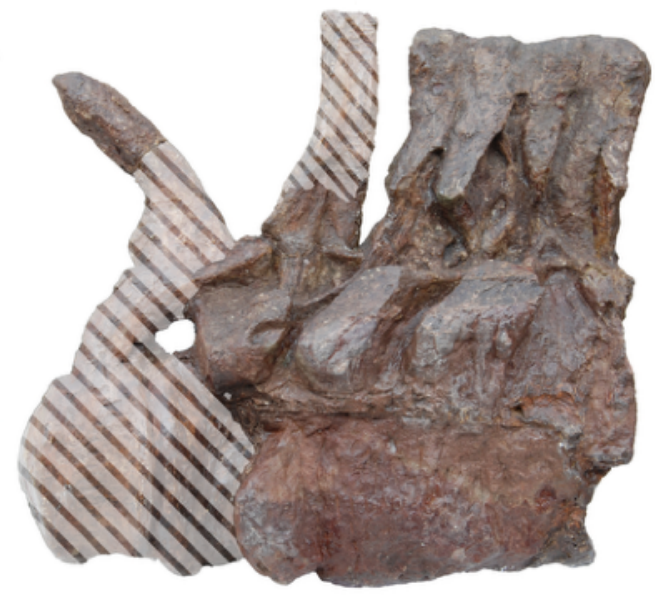

B

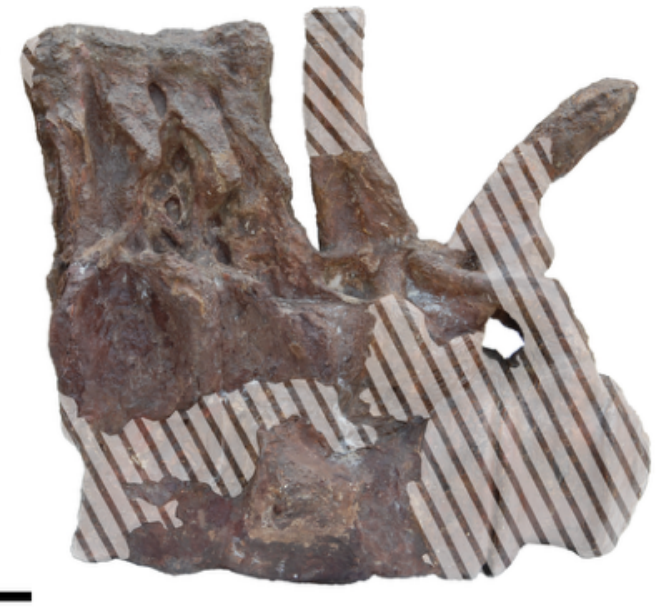

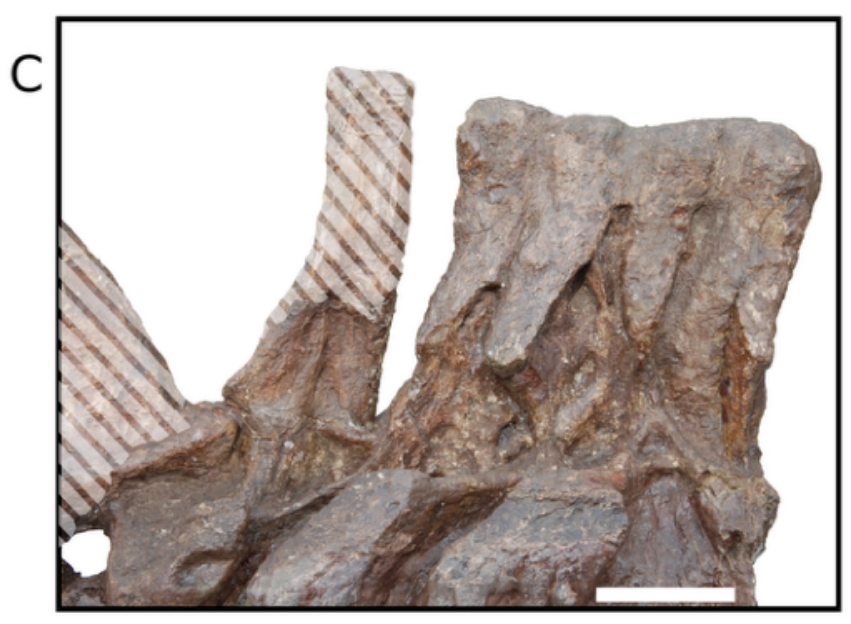

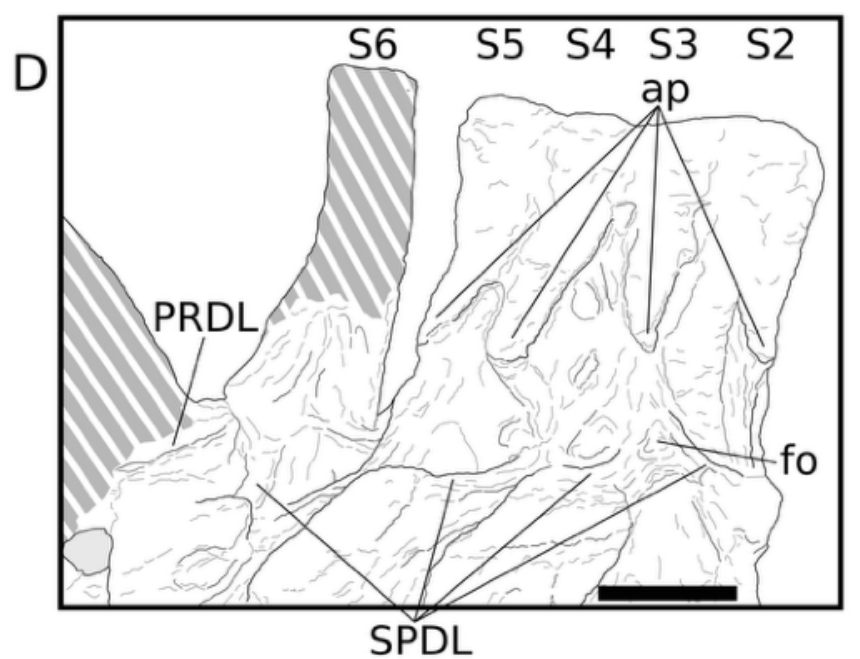

Figure 15. Sacral vertebrae 2-6 and caudal vertebra 1 of Klamelisaurus gobiensis (IVPP V9492). A, right lateral view; B, left lateral view; C, close-up of right neural arches of sacral vertebrae 26; D, schematic of close-up of right neural arches of sacral vertebrae 2-6. Abbreviations: ap, aliform process; fo, fossa; PRDL, prezygodiapophyseal lamina; S, sacral; SPDL, spinodiapophyseal lamina. Striped regions indicate reconstruction. Scale bar $=10 \mathrm{~cm}$. 


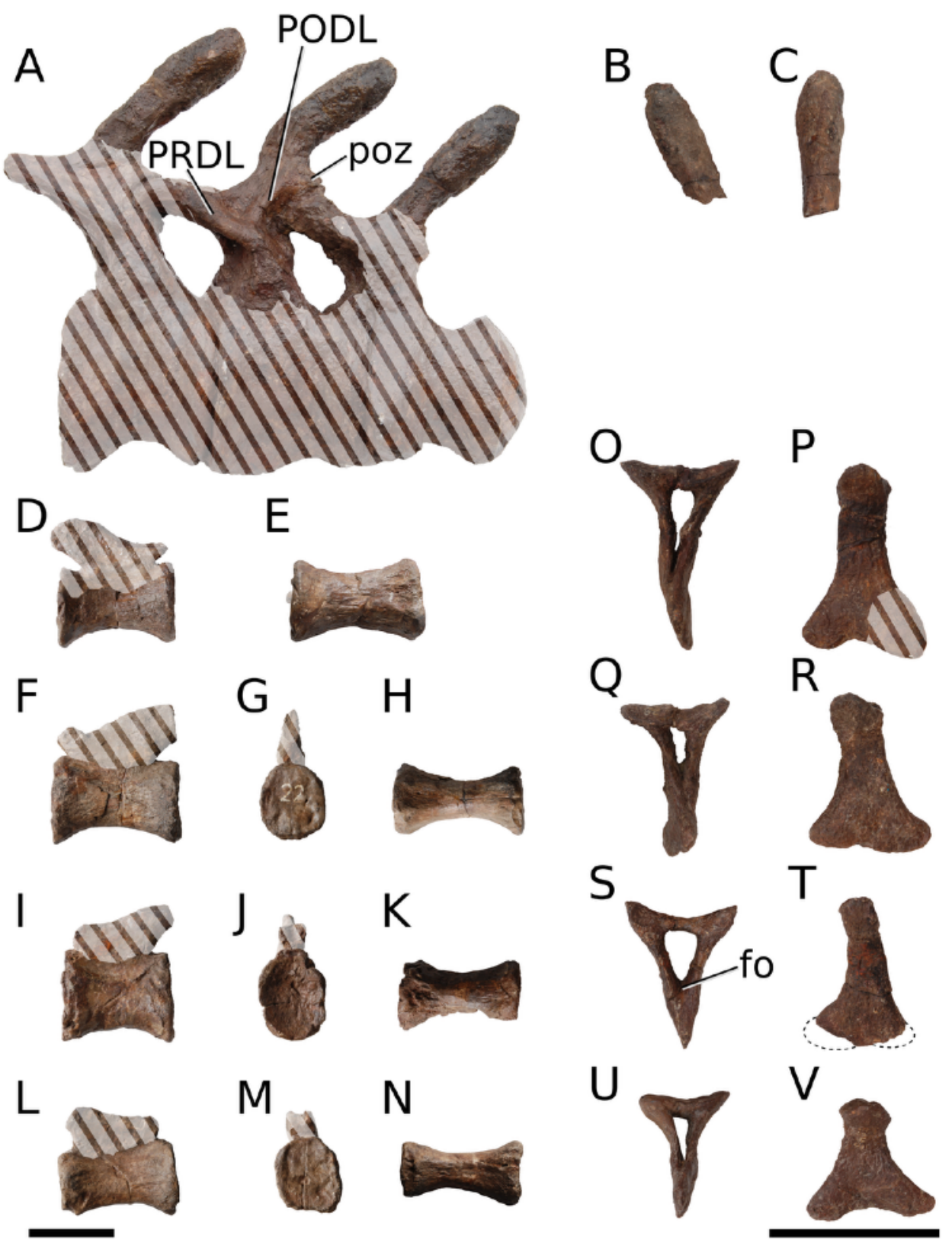

Figure 16. Caudal vertebrae of Klamelisaurus gobiensis (IVPP V9492). A, caudal vertebrae 2-4 in left lateral view; B, right lateral view of neural spine of caudal 8; C, posterior view of neural spine of caudal 8; D, right lateral view of centrum of caudal 19; E, ventral view of centrum of caudal 19. Centrum of caudal 22 in $(F)$ lateral view, $(G)$ posterior view and $(H)$ ventral view. Centrum of caudal 24 in (I) left lateral view, $(\mathrm{J})$ anterior view and $(\mathrm{K})$ ventral view. Centrum of caudal 26 in (L) right lateral view, $(\mathrm{M})$ anterior view and $(\mathrm{N})$ ventral view. Chevron 26 in (O) anterior view and (P) left lateral view. Chevron 28 in $(\mathrm{Q})$ anterior view and $(\mathrm{R})$ left lateral view. Chevron 29 in (S) anterior view and (T) right lateral view. Chevron 30 in (U) anterior view and (V) left lateral view. Abbreviations: fo, fossa; PODL, postzygodiapophyseal lamina; poz, postzygapophysis; PRDL, prezygodiapophyseal lamina. Striped regions indicate reconstruction. Scale bars $=10 \mathrm{~cm}$. 


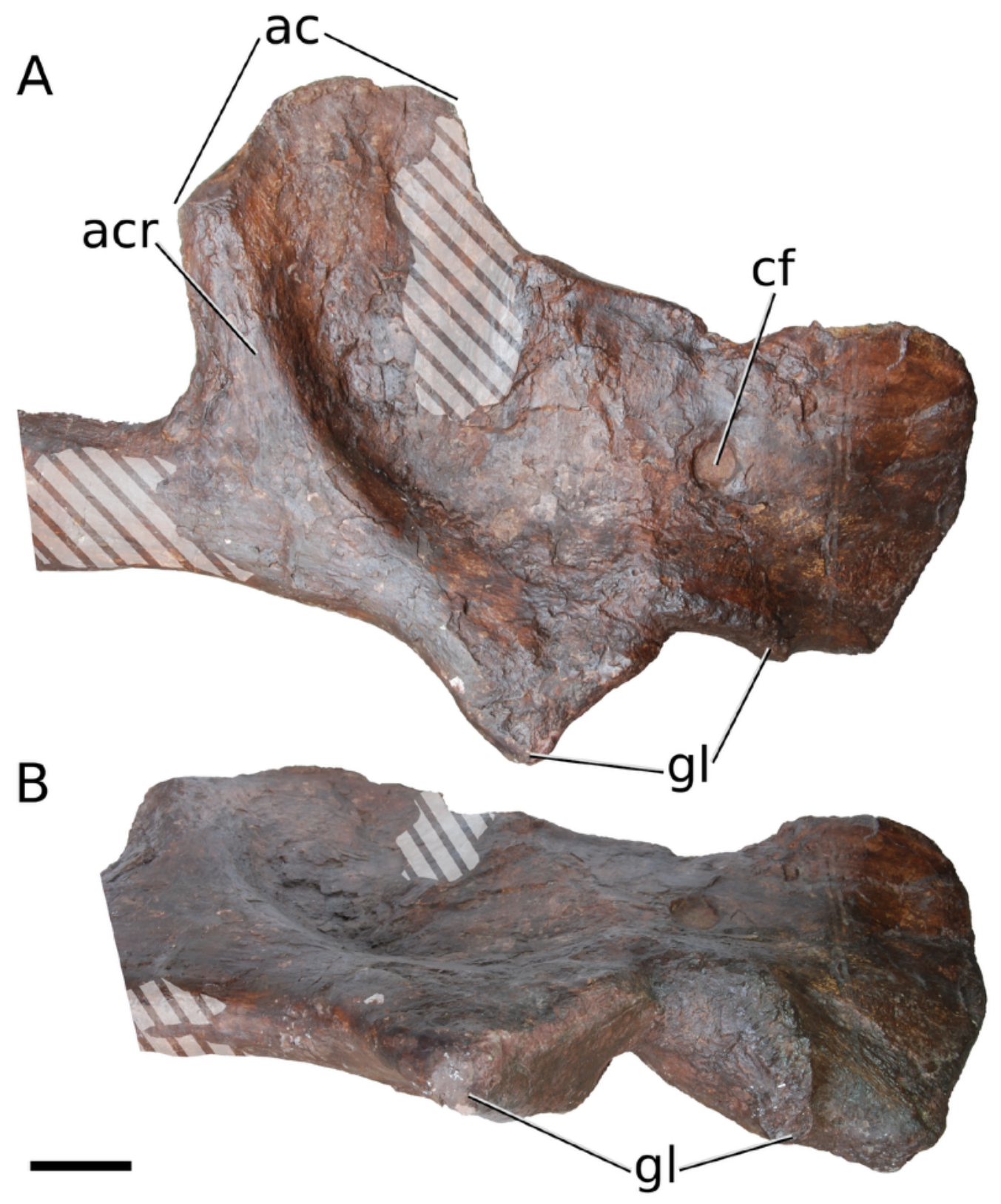

Figure 17. Right scapulocoracoid of Klamelisaurus gobiensis (IVPP V9492). A, lateral view; B, ventrolateral view. Note the medial deflection of the scapular glenoid. Regions of the scapular blade beyond the point depicted are morphologically uninformative and largely reconstructed. Abbreviations: ac, acromion; acr, acromial ridge; cf, coracoid foramen; gl, glenoid. Striped regions indicate reconstruction. Scale bar $=10 \mathrm{~cm}$. 


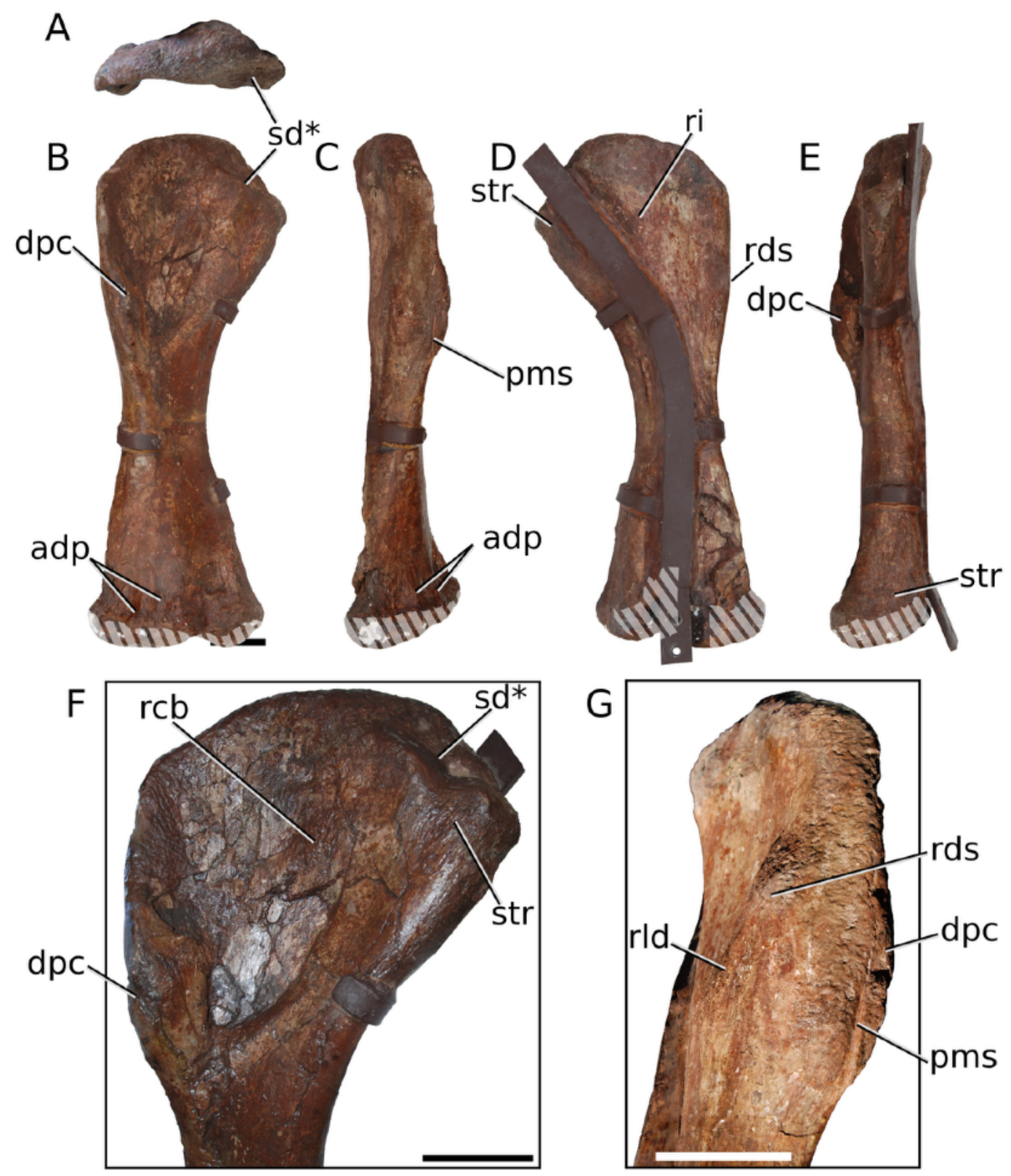

Figure 18. Right humerus of Klamelisaurus gobiensis (IVPP V9492). A, proximal view; B, anterior view; C, lateral view; D, posterior view; E, medial view; F, close-up of proximal anterior fossa, in anterior and slightly medial view; $G$, close-up of posterior and lateral edge of the humerus, at the level of the deltopectoral crest. Abbreviations: adp, anterodistal process; dpc, deltopectoral crest; pms, paramarginal sulcus; rcb, low rugosity for m. coracobrachialis; rds, rugosity for $\mathrm{m}$. deltoideus scapularis; ri, ridge; rld, rugosity for m. latissimus dorsi; sd, shelf-like depression; str, striations. Asterisk indicates an autapomorphy. Striped regions indicate reconstruction. Scale bars $=10 \mathrm{~cm}$. 


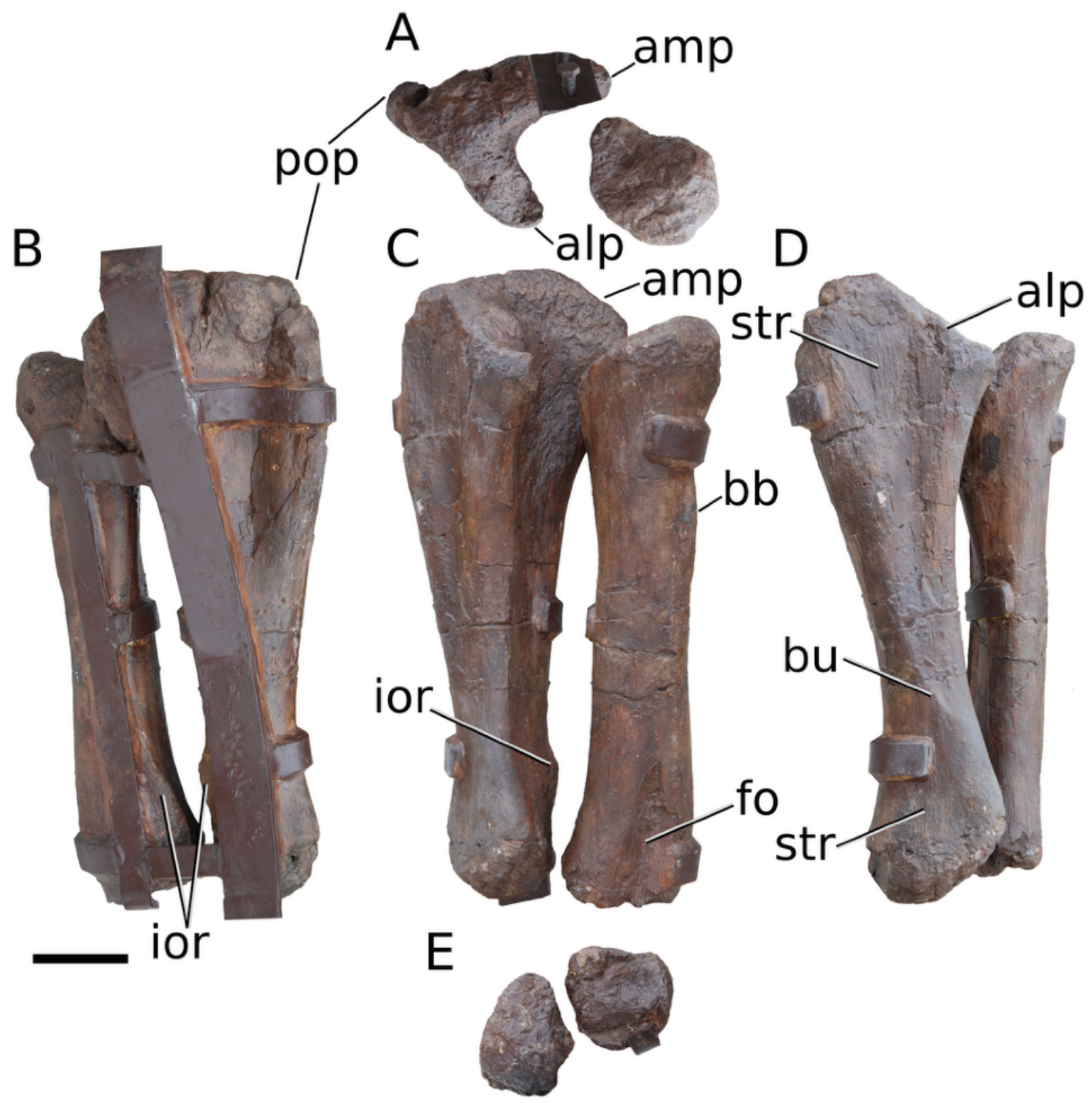

Figure 19. Right ulna and radius of Klamelisaurus gobiensis (IVPP V9492). Orientational descriptors for B-D reflect the positioning of the ulna; as described in the text, the radius has been rotated slightly out of proper anatomical relation to the ulna. A, proximal view (as positioned within armature); B, posteromedial view; $\mathrm{C}$, anterolateral view; $\mathrm{D}$, posterolateral view; E, distal view. Abbreviations: alp, anterolateral process; amp, anteromedial process; bb, bulge for the m. biceps brachii; bu, bump; fo, fossa; ior, interosseous ridge; pop, posterior process; str, striations. Scale bar $=10 \mathrm{~cm}$. 


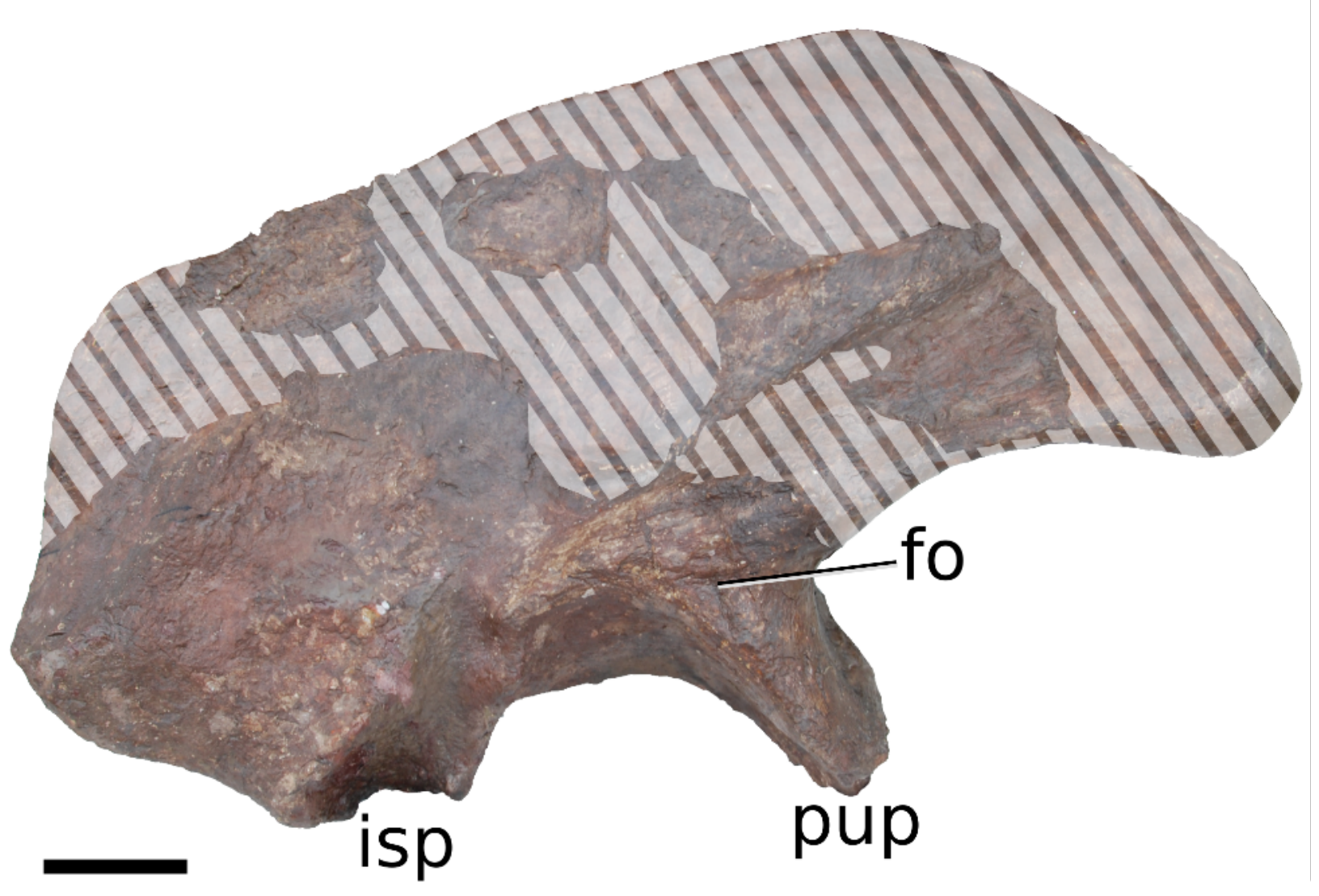

Figure 20. Right ilium of Klamelisaurus gobiensis (IVPP V9492) in lateral view. Abbreviations: fo, fossa; isp, ischial peduncle; pup, pubic peduncle. Striped regions indicate reconstruction. Scale bar $=10 \mathrm{~cm}$. 
A

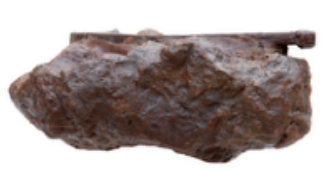

B
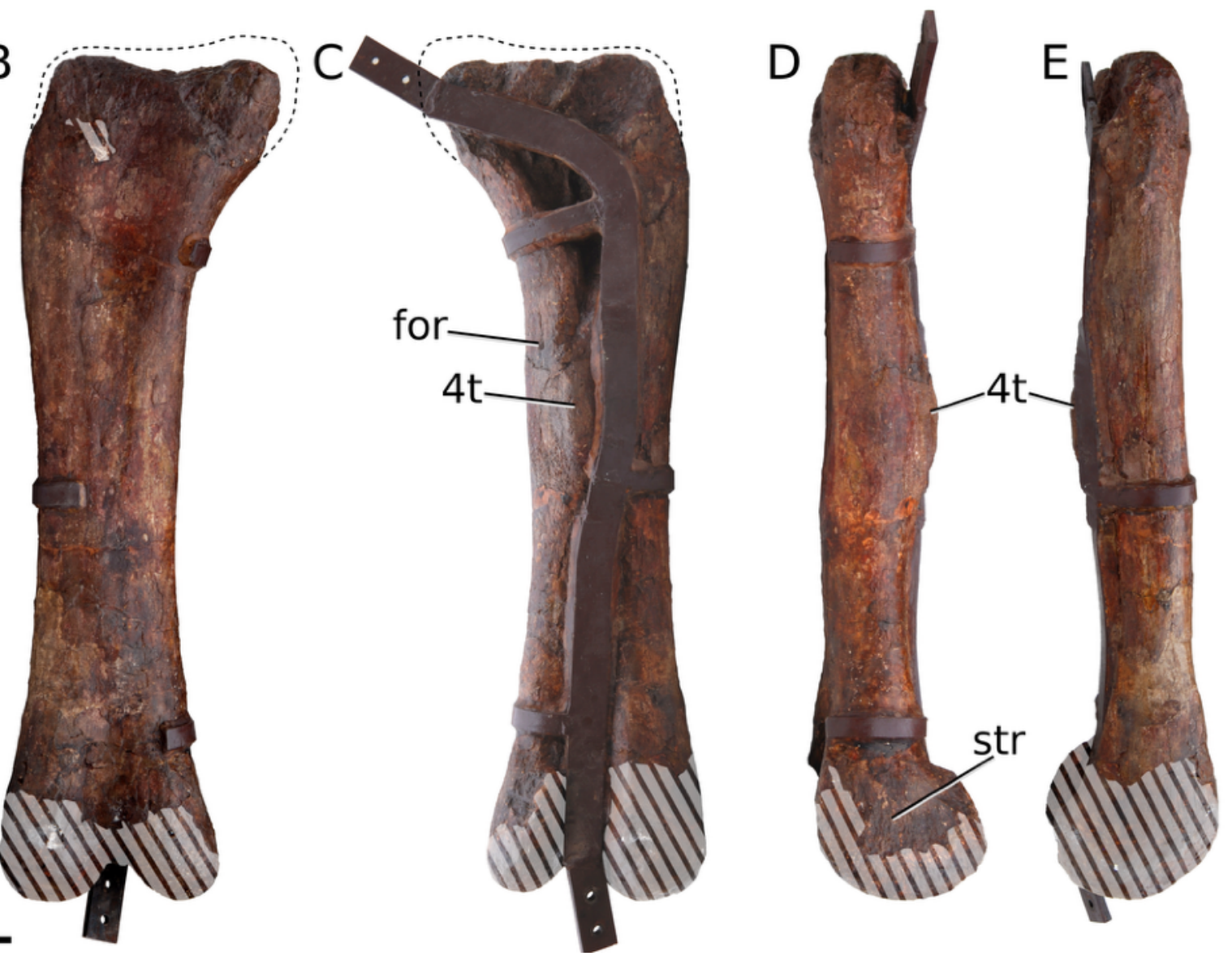

Figure 21. Right femur of Klamelisaurus gobiensis (IVPP V9492). A, proximal view; B, anterior view; C, posterior view; $\mathrm{D}$, medial view; $\mathrm{E}$, lateral view. Abbreviations: 4t, fourth trochanter; for, foramen; str, striations. Striped regions indicate reconstruction. Scale bar $=10 \mathrm{~cm}$. 


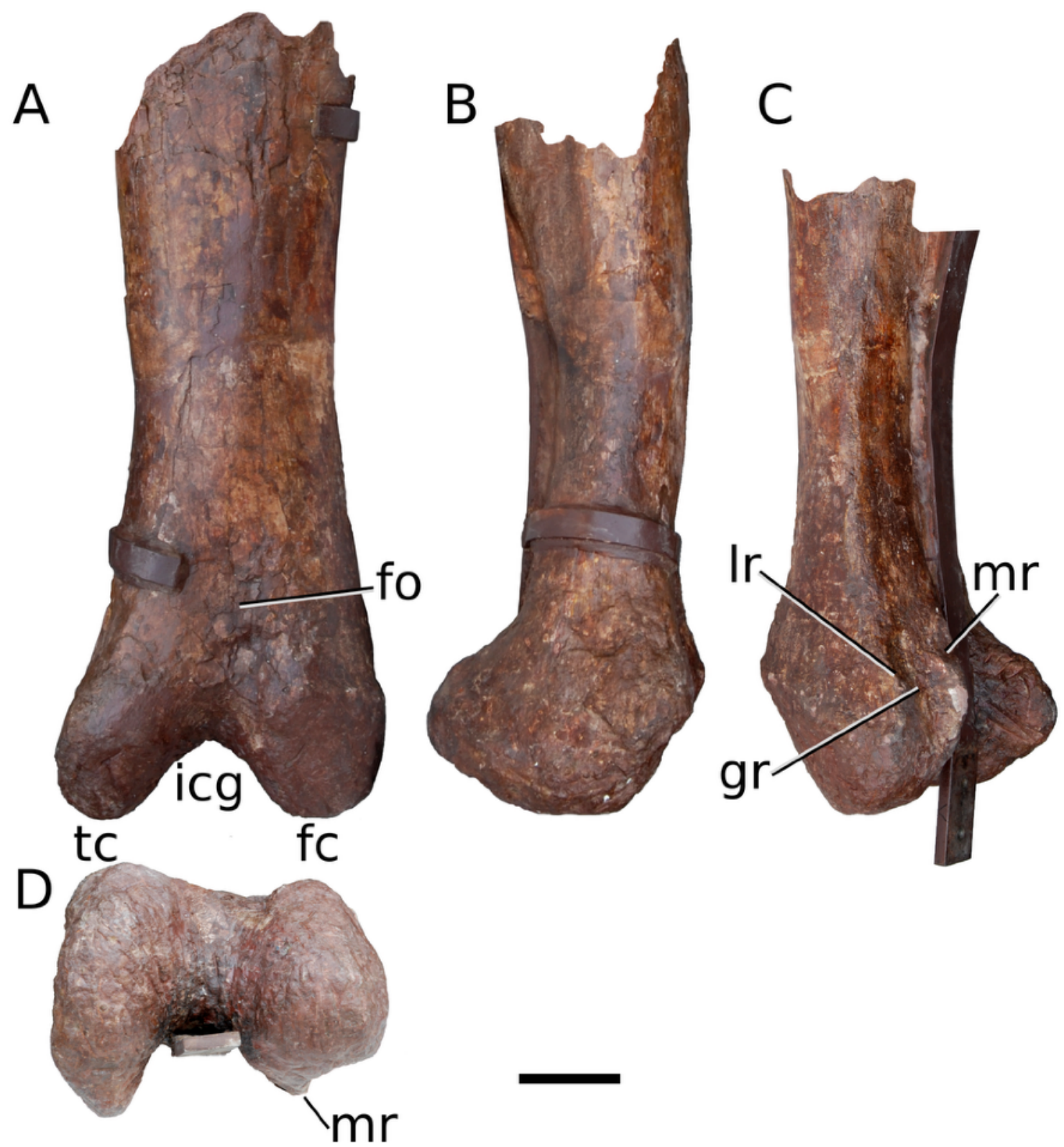

Figure 22. Left femur of Klamelisaurus gobiensis (IVPP V9492). Only the distal half of the element is unreconstructed. A, anterior view; B, medial view; $\mathrm{C}$, posterolateral view; $\mathrm{D}$, distal view. Abbreviations: fc, fibular condyle; fo, fossa; gr, groove; icg, intercondylar groove; lr, lateral ridge; $\mathrm{mr}$, medial ridge; tc, tibial condyle. Striped regions indicate reconstruction. Scale bar $=10 \mathrm{~cm}$. 


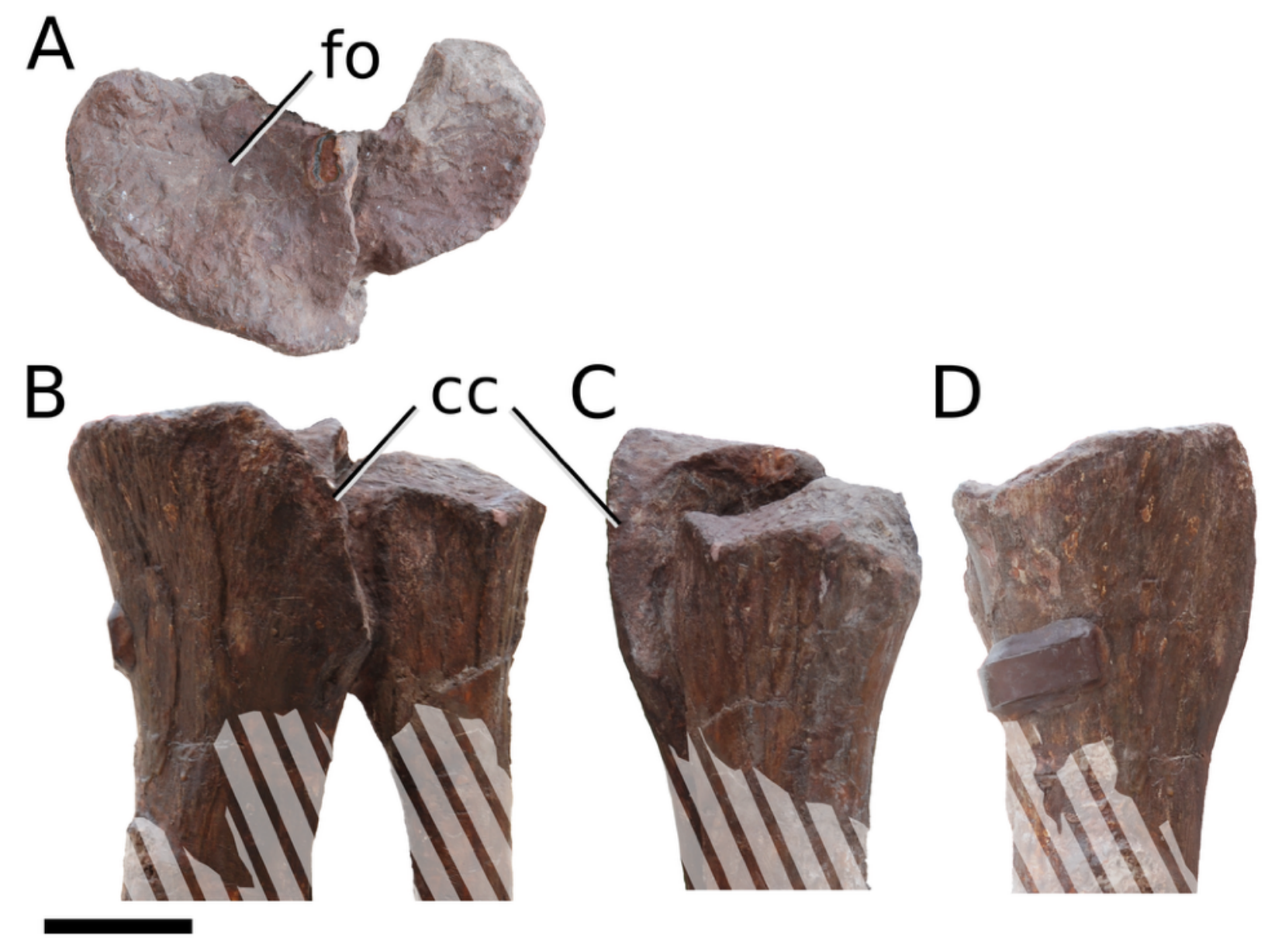

Figure 23. Left tibia and fibula of Klamelisaurus gobiensis (IVPP V9492). A, proximal view; B, anterior view; C, lateral view; D, medial view. Abbreviations: cc: cnemial crest; fo, fossa. Striped regions indicate reconstruction. Scale bar $=10 \mathrm{~cm}$. 


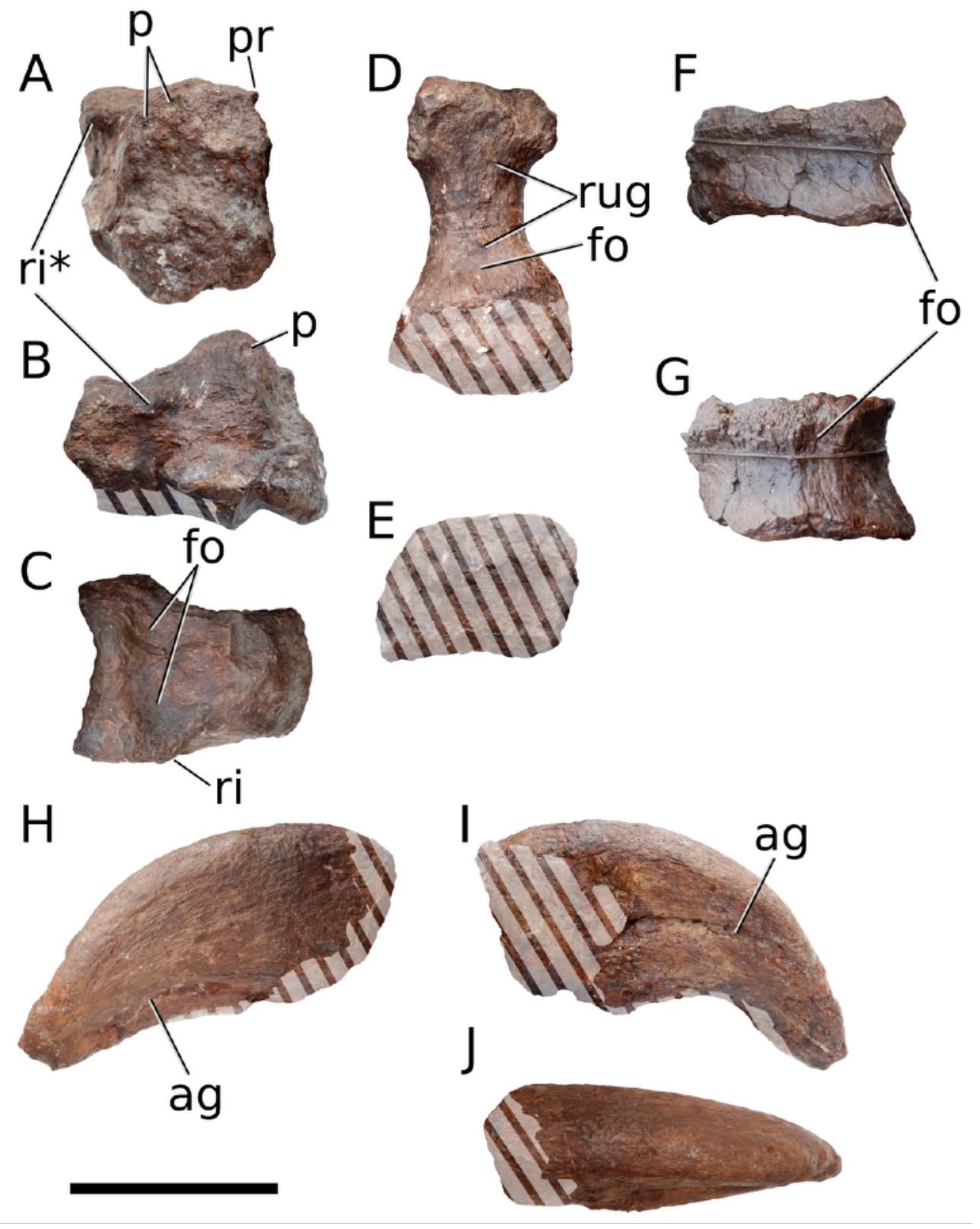

Figure 24. Pedal elements of Klamelisaurus gobiensis (IVPP V9492). Right metatarsal I in (A) proximal, (B) medial and (C) dorsal views. ?Right metatarsal II or III in (D) dorsal and (E) proximal views. ?Left pedal phalanx ?III-1 in (F) dorsal and (G) dorsolateral views. Right pedal ungual in $(\mathrm{H})$ medial, (I) lateral and (J) dorsal views. Abbreviations: ag, attachment groove; fo, fossa; p, pit; pr, projection; ri, ridge; rug, rugosity. Asterisk indicates an autapomorphy. Striped regions indicate reconstruction. Scale bar $=10 \mathrm{~cm}$. 


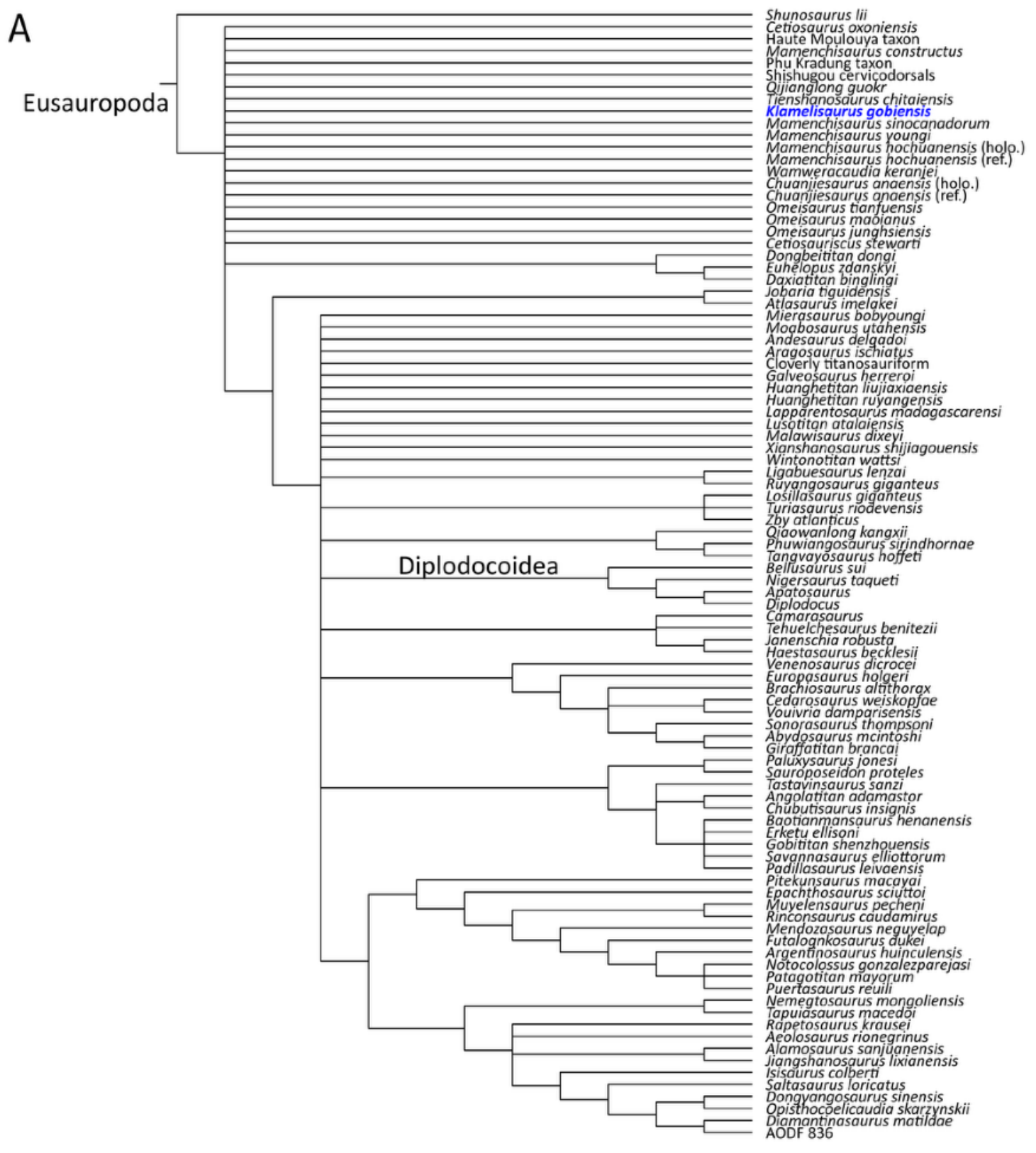




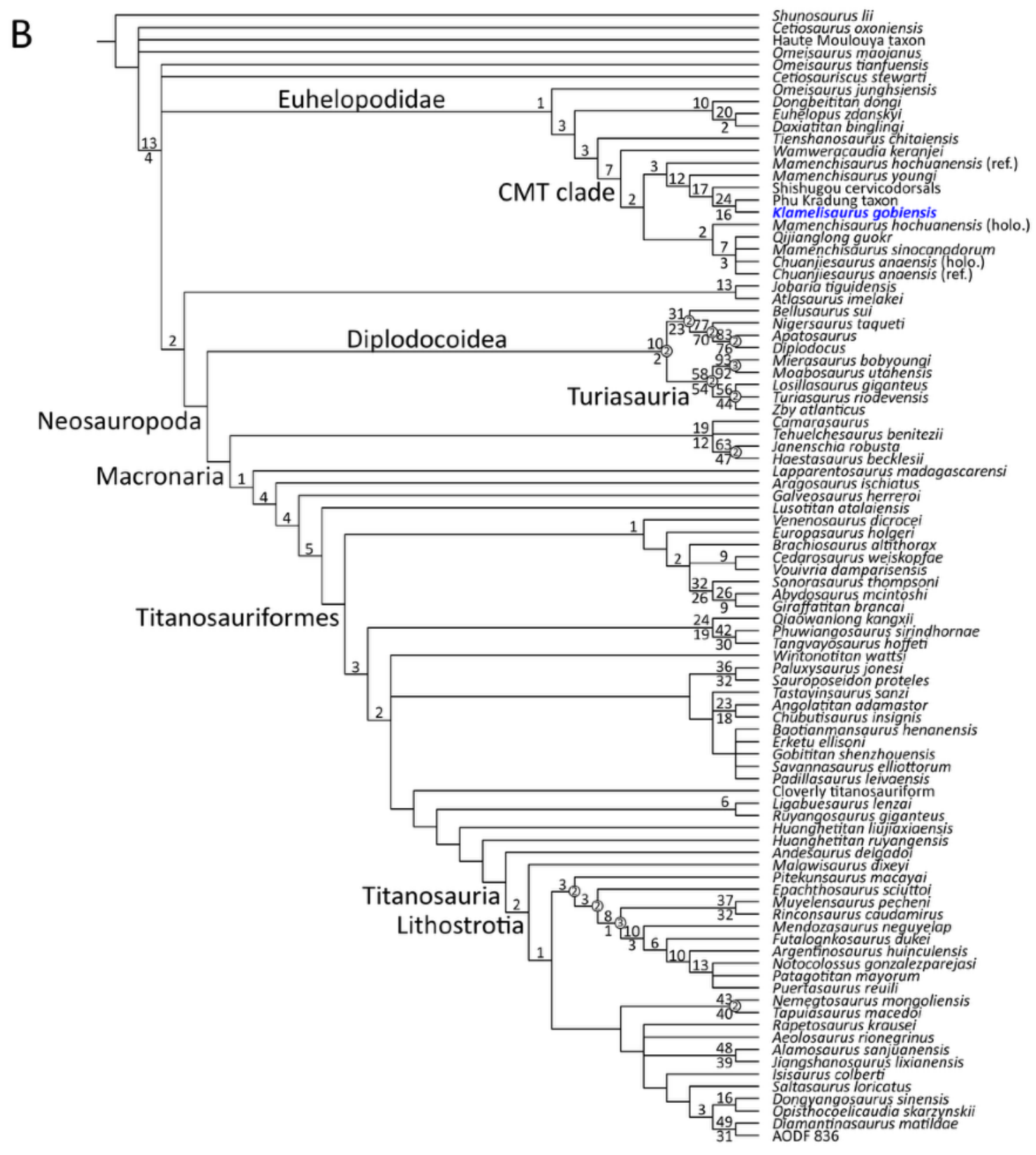

Figure 25. Results of the equal-weights parsimony analysis of the GEA matrix. A, strict consensus of 200,000 MPTs; B, reduced strict consensus, following a posteriori pruning of Mamenchisaurus constructus and Xianshanosaurus. 'CMT clade' marks the node subtending Core Mamenchisaurus-like Taxa. Values above nodes are absolute symmetric resampling frequencies, and values below nodes are GC frequencies; only positive frequencies are shown. Decay index values $>1$ are depicted in circles on nodes. Support values are only shown for (B). 


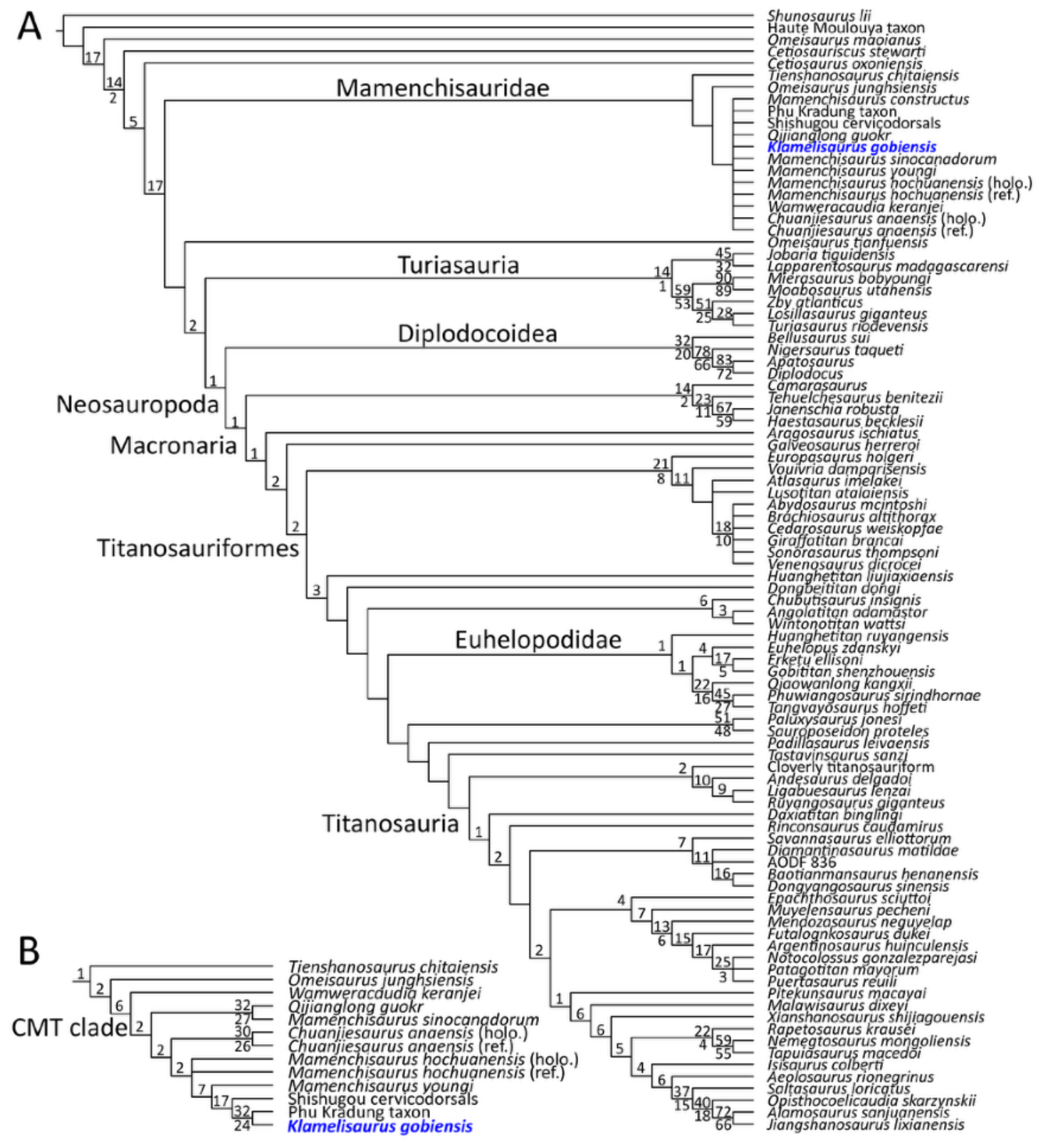

Figure 26. Results of the extended implied-weights parsimony analysis of the GEA matrix. A, strict consensus of 30 MPTs; B, reduced strict consensus of Mamenchisauridae, following a posteriori pruning of Mamenchisaurus constructus. 'CMT clade' marks the node subtending Core Mamenchisaurus-like Taxa. Values above nodes are absolute symmetric resampling frequencies, and values below nodes are GC frequencies; only positive frequencies are shown. 


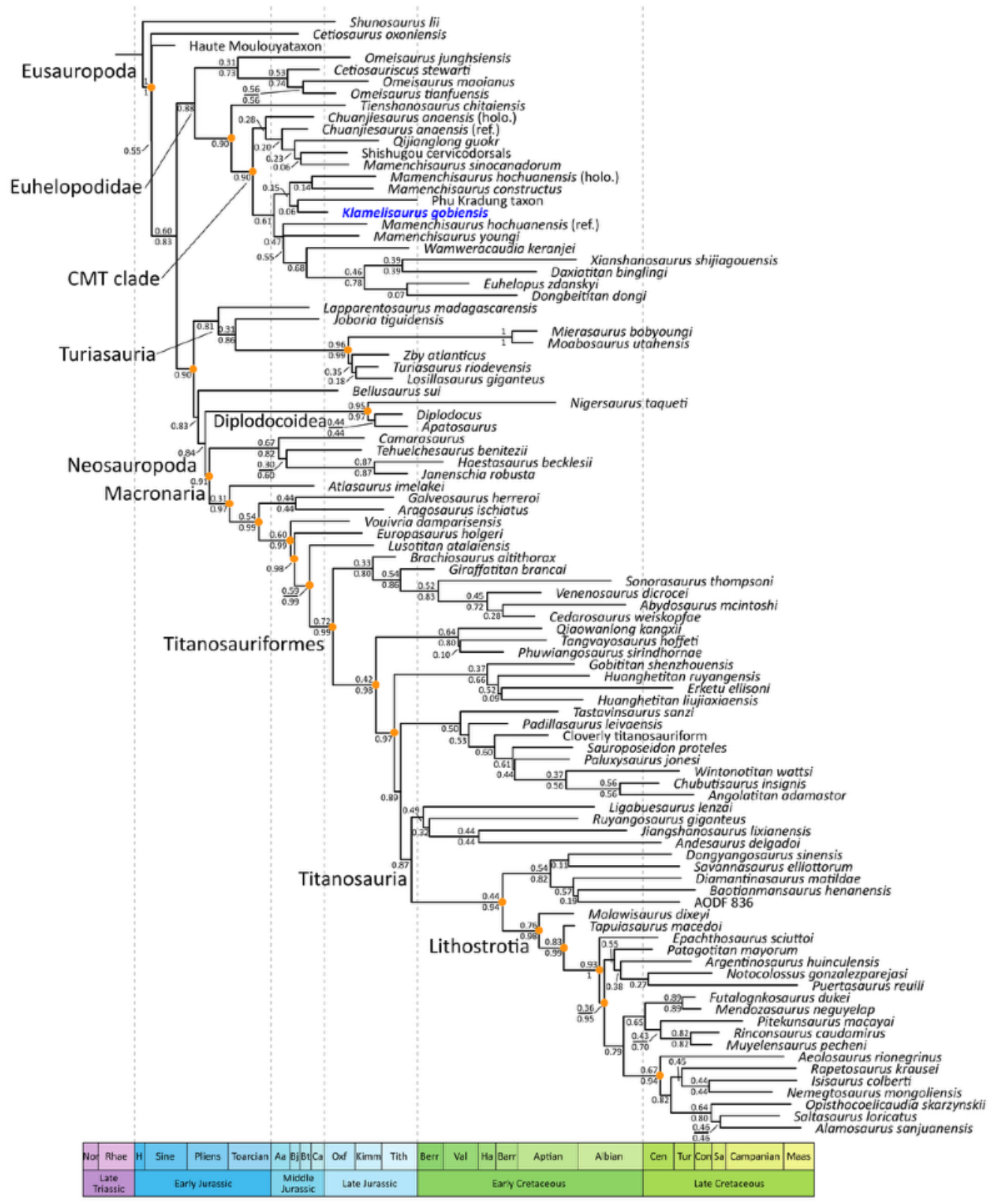

Figure 27. Results of the time-calibrated Bayesian analysis of the GEA matrix. 'CMT clade' marks the node subtending Core Mamenchisaurus-like Taxa. Posterior probabilities (0.30) are given above nodes, and TPE values given below nodes. Orange circles indicate TPE $\geq 0.90$. 


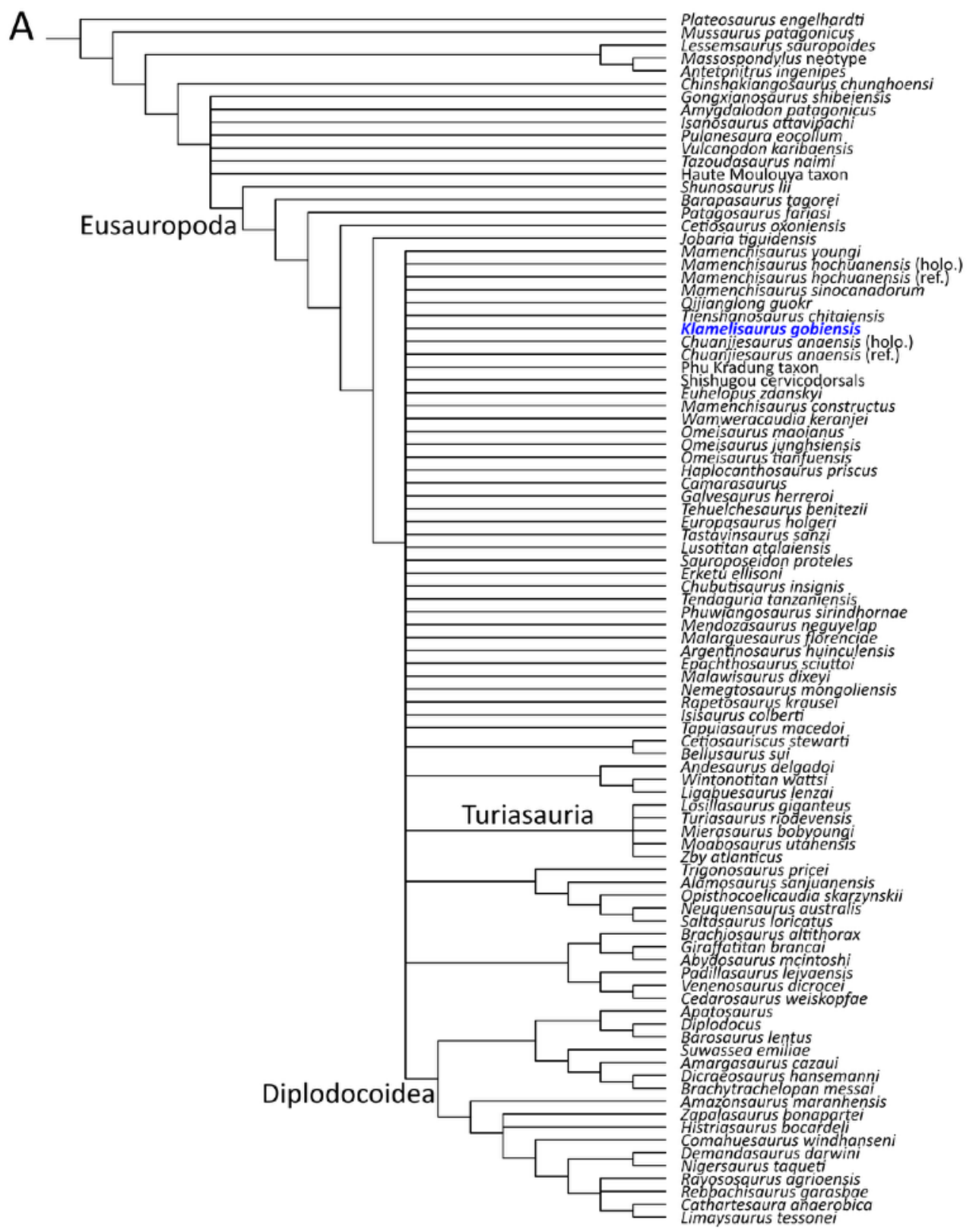




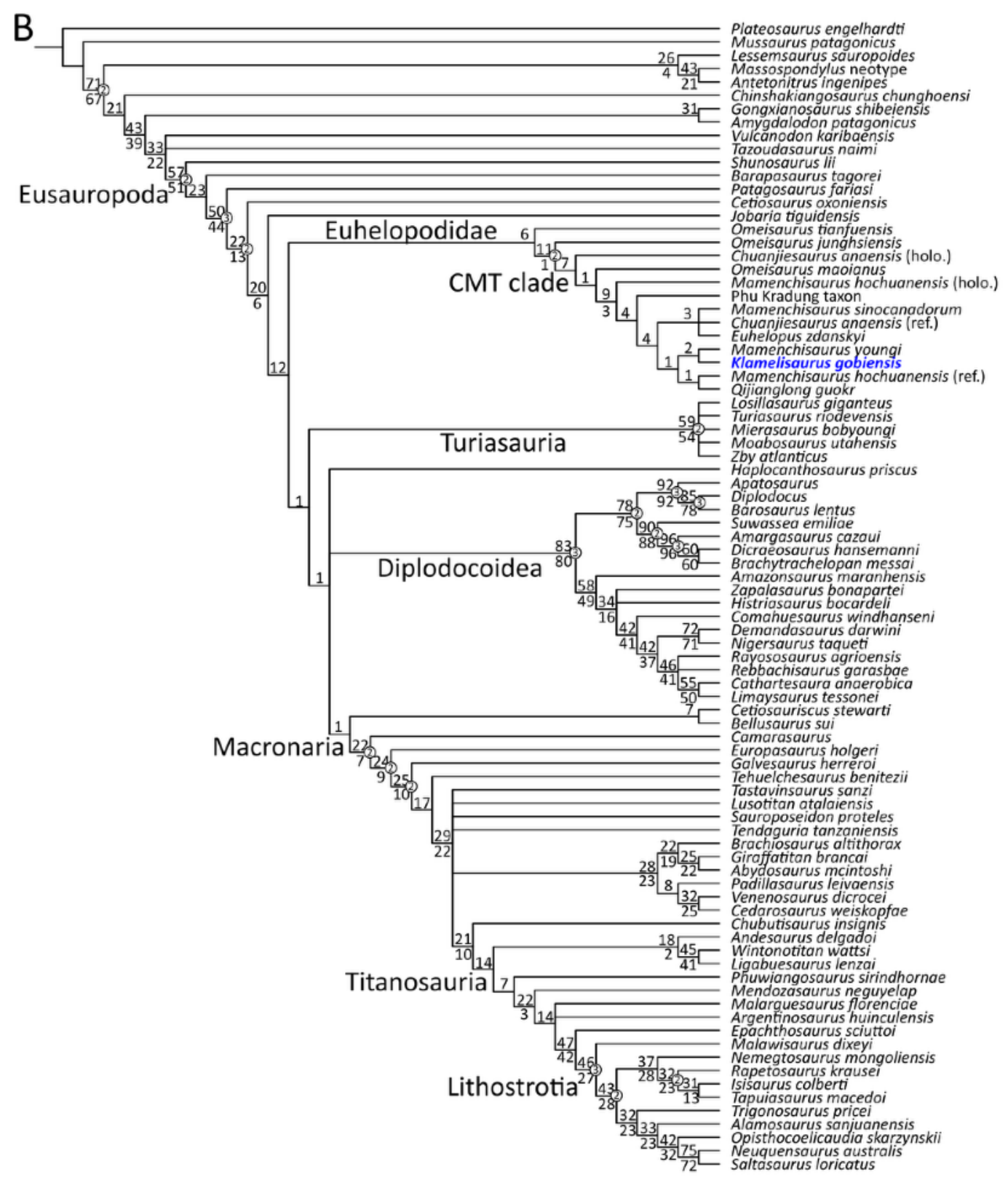

Figure 28. Results of the equal-weights parsimony analysis of the CEA matrix. A, strict consensus of 200,000 MPTs; B, reduced strict consensus, following a posteriori pruning of Pulanesaura, Isanosaurus, the Haute Moulouya taxon, Tienshanosaurus, Mamenchisaurus constructus, Wamweracaudia, the Shishugou cervicodorsal vertebrae and Erketu. 'CMT clade' marks the node subtending Core Mamenchisaurus-like Taxa. Values above nodes are absolute symmetric resampling frequencies, and values below nodes are GC frequencies; only positive frequencies are shown. Decay index values $>1$ are depicted in circles on nodes. Support values are only shown for (B). 


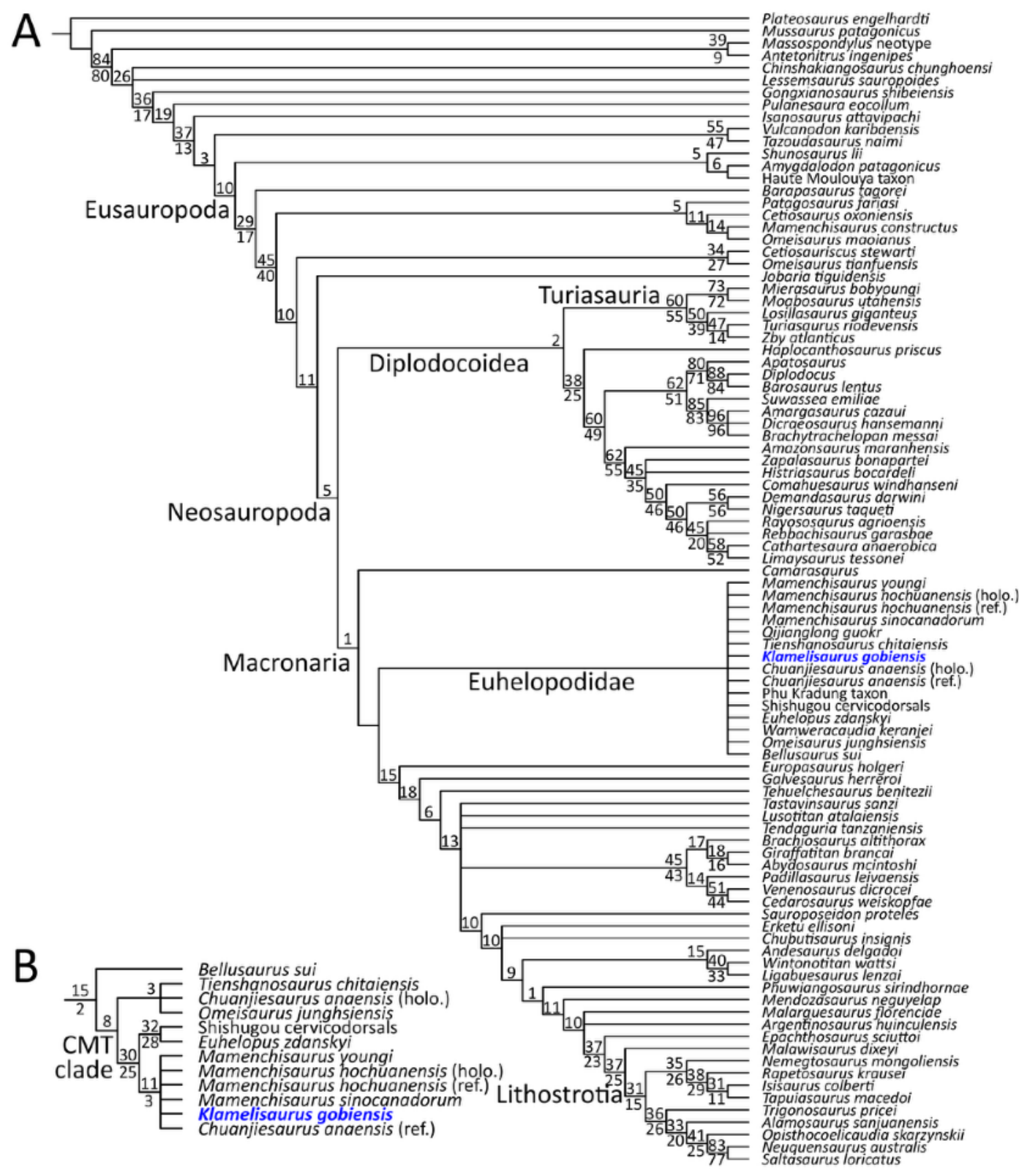

Figure 29. Results of the extended implied-weights parsimony analysis of the CEA matrix. A, strict consensus of 24,925 MPTs; B, reduced strict consensus of Euhelopodidae, following a posteriori pruning of Wamweracaudia, the Phu Kradung taxon, and Qijianglong. 'CMT clade' marks the node subtending Core Mamenchisaurus-like Taxa. Values above nodes are absolute symmetric resampling frequencies, and values below nodes are GC frequencies; only positive frequencies are shown. 


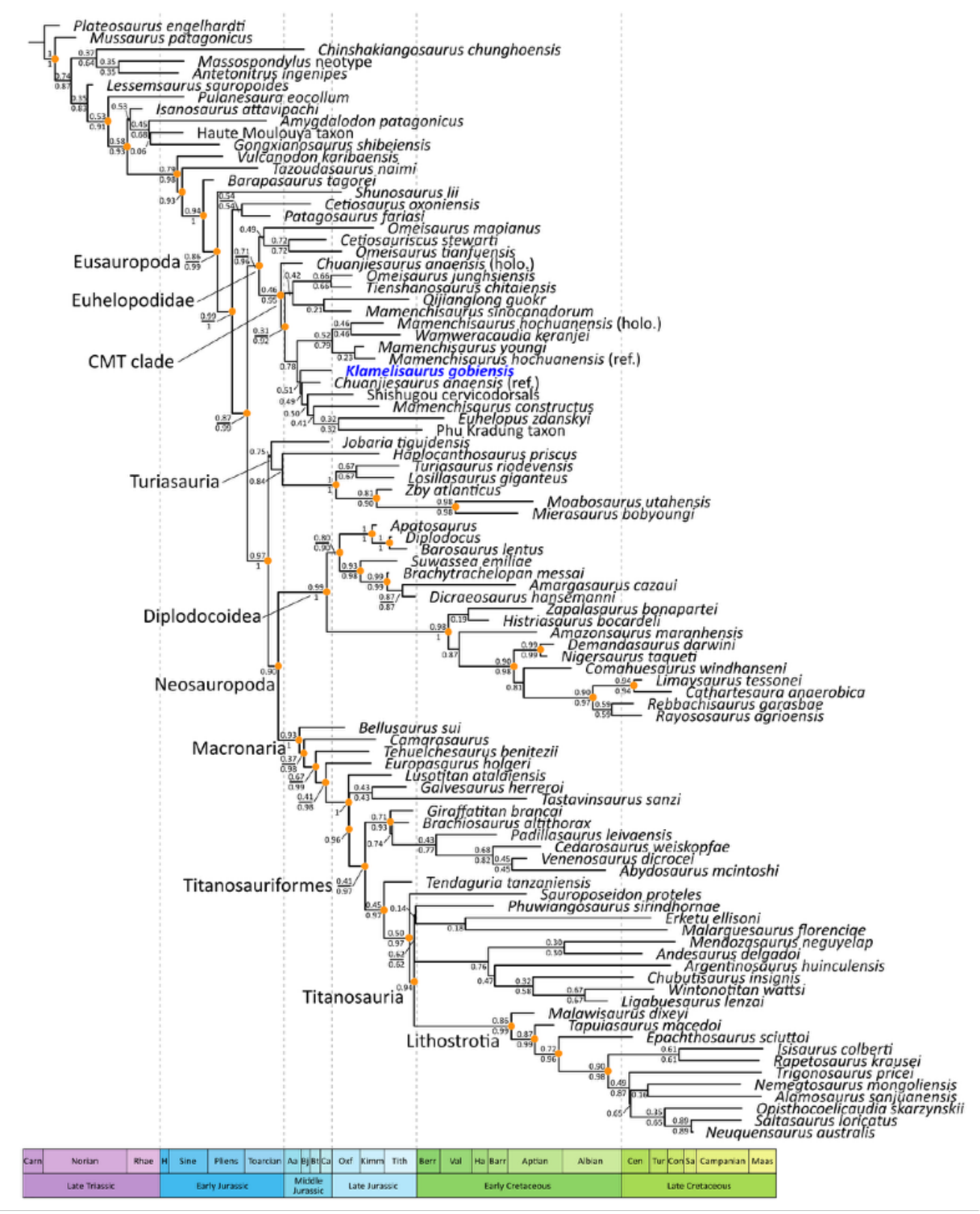

Figure 30. Results of the time-calibrated Bayesian analysis of the CEA matrix. 'CMT clade' marks the node subtending Core Mamenchisaurus-like Taxa. Posterior probabilities (0.30) are given above nodes, and TPE values given below nodes. Orange circles indicate TPE $\geq 0.90$. 

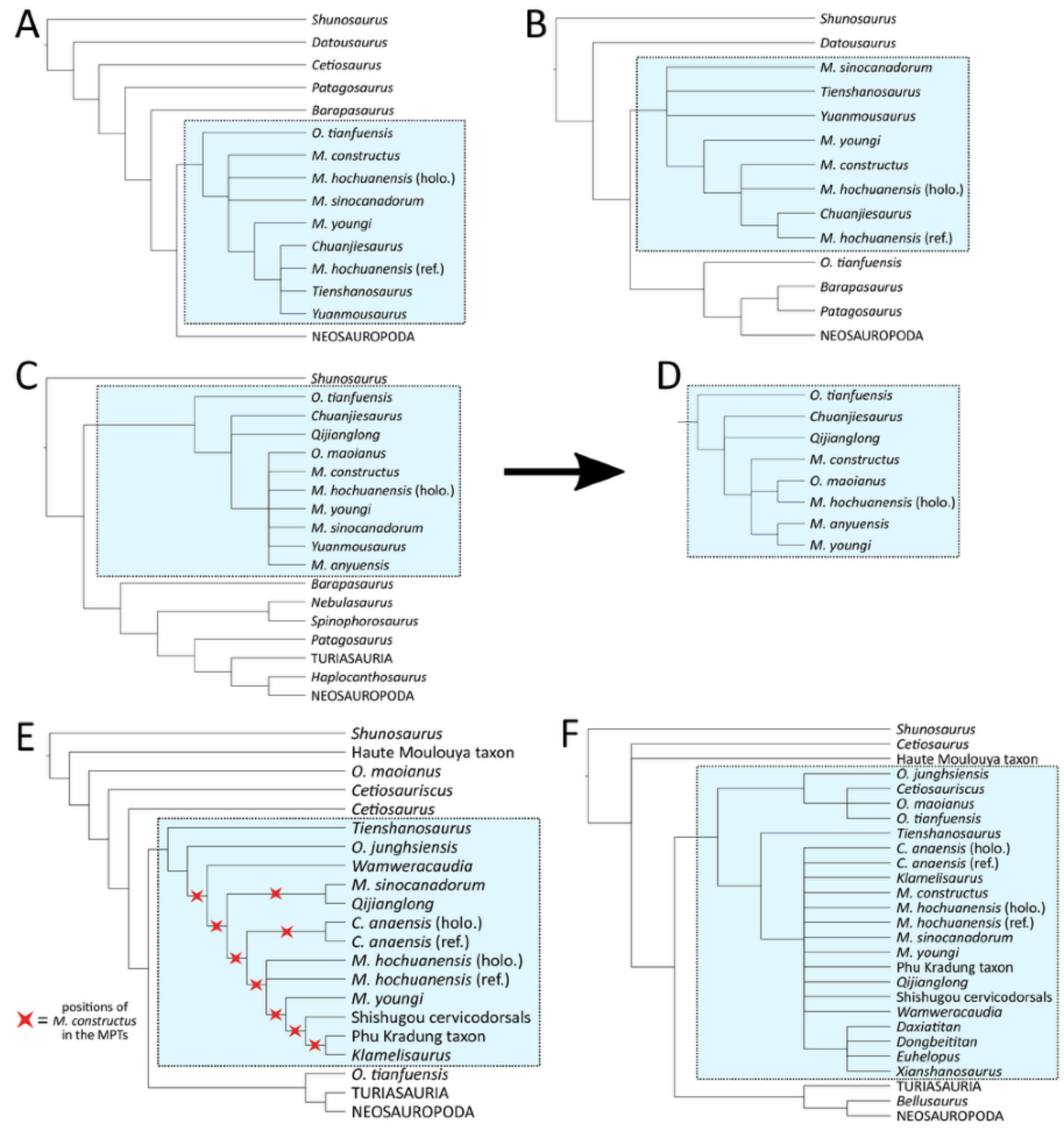

Figure 31. Evolutionary relationships within Mamenchisauridae/Euhelopodidae. Blue boxes outline Mamenchisauridae (A-E) and Euhelopodidae (F). A, strict consensus topology from analysis of the modified matrix of Upchurch et al. (2004) by Sekiya (2011); B, strict consensus topology from analysis of the modified matrix of Wilson (2002) by Sekiya (2011); C, strict consensus topology from analysis of Xing et al. (2015); D, reduced strict consensus topology of Mamenchisauridae from Xing et al. (2015), following a posteriori pruning of Mamenchisaurus sinocanadorum and Yuanmousaurus; E, reduced strict consensus from implied-weights parsimony analysis of the GEA matrix (this study), following a posteriori pruning of Mamenchisaurus constructus; F, results from the time-calibrated Bayesian analysis of the GEA matrix (this study); nodes with less than 0.70 TPE support have been collapsed. 


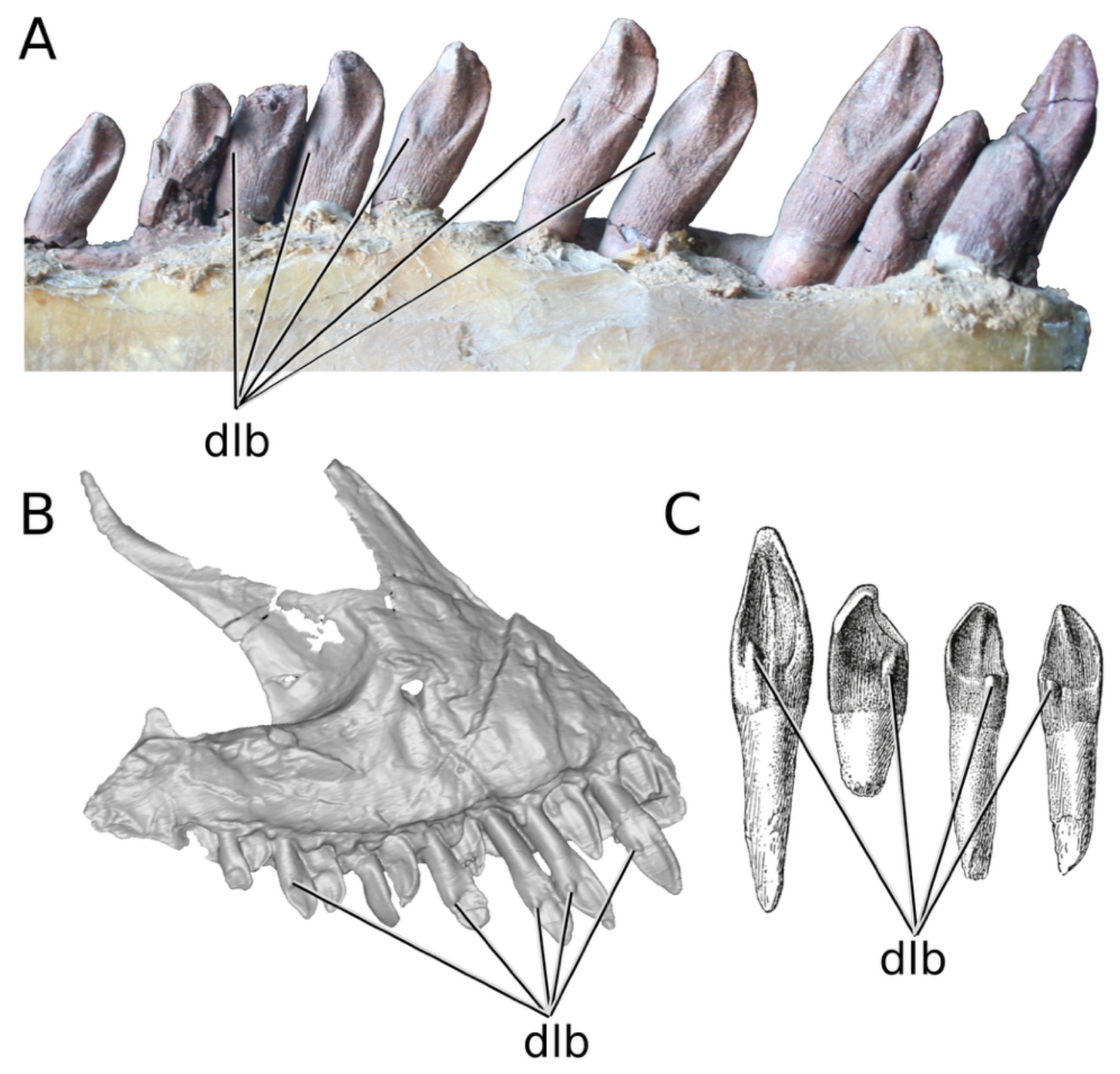

Figure 32. Distolingual boss in the dentitions of Mamenchisaurus sinocanadorum and Euhelopus. A, medial view of the left dentary of Mamenchisaurus sinocanadorum (IVPP V10603); B, medial view of left maxilla of Euhelopus (PMU 24705/1a-b); C, isolated teeth of Euhelopus, modified from Wiman (1929, pl. II). Abbreviation: dlb, distolingual boss. Not to scale. 

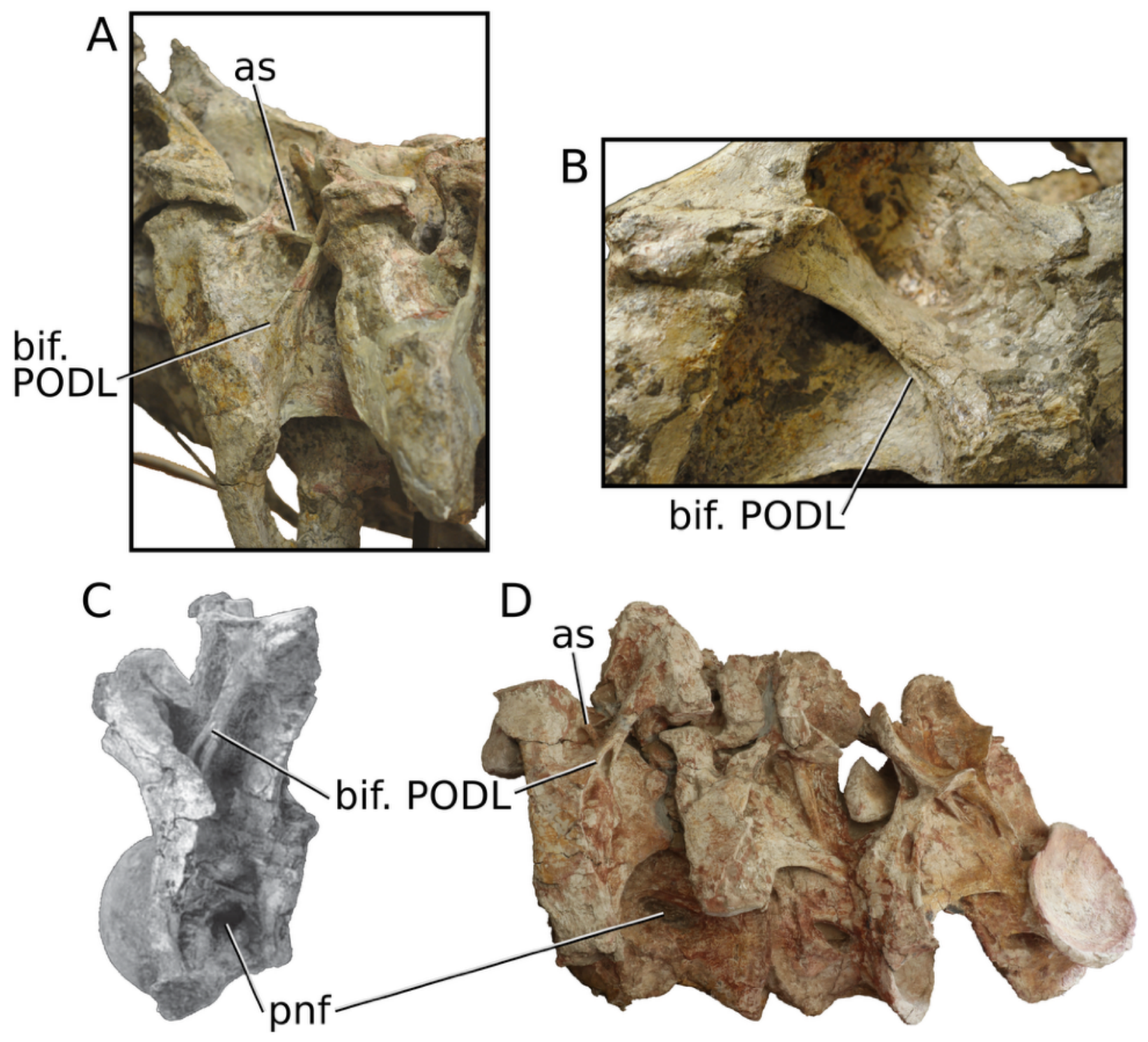

Figure 33. Ventral bifurcation of the postzygodiapophyseal lamina in several East Asian eusauropods. A, cervical 17 of Euhelopus zdanskyi (PMU 233) in left posterolateral view; B, right postzygodiapophyseal lamina of cervical 15 of Euhelopus zdanskyi (PMU 233) in right lateral view; $C$, isolated posterior cervical vertebra of the Phu Kradung taxon in left lateral view, modified from Suteethorn et al. (2013, fig. 3); D, uncatalogued series of cervicodorsal vertebrae from the Shishugou Formation (IVPP field jacket number WCW-05-35). Abbreviations: as, accessory strut; bif. PODL, ventrally bifurcated postzygodiapophyseal lamina; pnf, pneumatic foramen. Images of Euhelopus cervical vertebrae used with permission of B. Kear. Not to scale. 

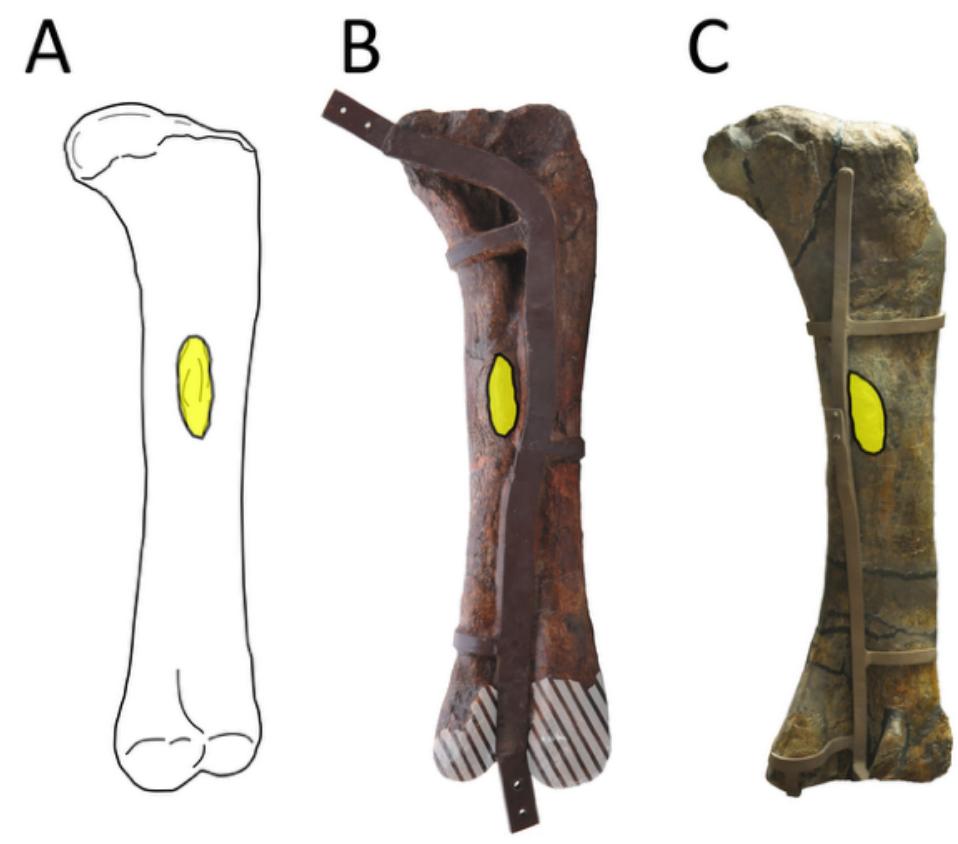

\section{$\mathrm{F}$}

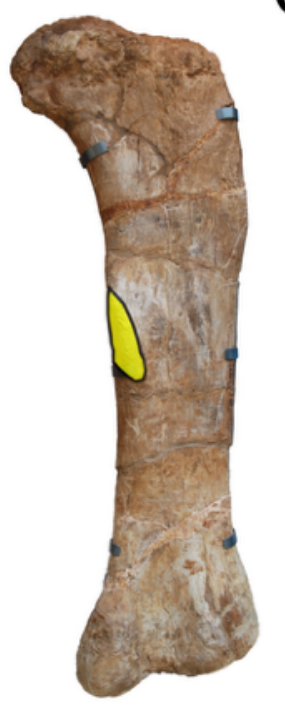

$G$

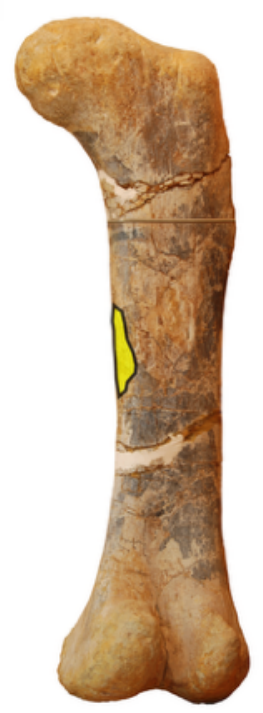

D

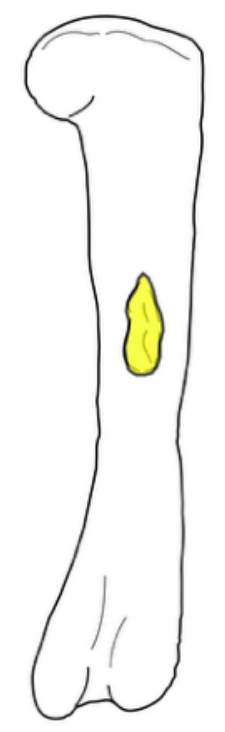

$\mathrm{E}$

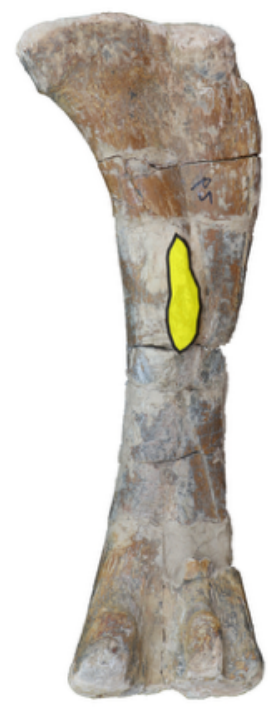

$\mathrm{H}$

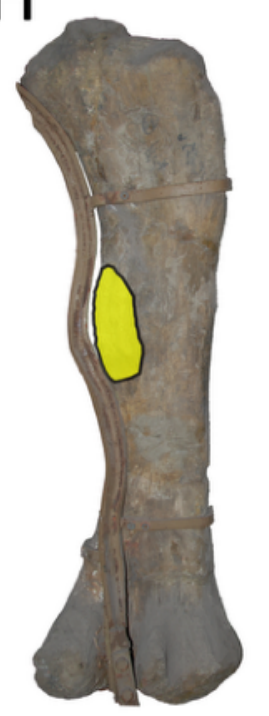

I

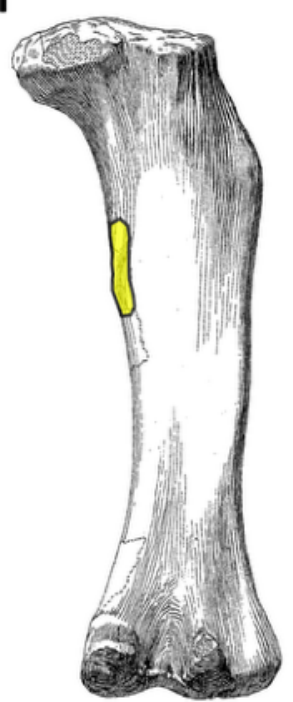

Figure 34. Variation in the position of the femoral fourth trochanter in various eusauropods. All femora are depicted in posterior view. The fourth trochanter (outlined) may be positioned on or near the posterior midline of the femur (A-E), at the medial margin of the femur, but without interrupting that margin $(\mathrm{F}, \mathrm{G})$, or projecting medially from the medial margin, such that it is visible in anterior view (H, I). A, left femur (reversed) of Shunosaurus lii, modified from Y. Zhang (1988, plate 13); B, right femur of Klamelisaurus gobiensis IVPP V9492; C, right femur of Euhelopus zdanskyi PMU 234; D, right femur of Mamenchisaurus youngi, modified from Ouyang \& Ye (2002, plate 14); E, left femur (reversed) of Bellusaurus sui IVPP V17768-62; F, right femur of Cetiosaurus oxoniensis OUMNH J.13615; G, left femur (reversed) of Aragosaurus ischiaticus ZH-2; H, left femur (reversed) of Omeisaurus tianfuensis ZDM T5701; I, right femur of Giraffatitan brancai, modified from Janensch (1961, fig. 1A of Beilage J). Image of Euhelopus in (C) used with permission of B. Kear. Not to scale. 


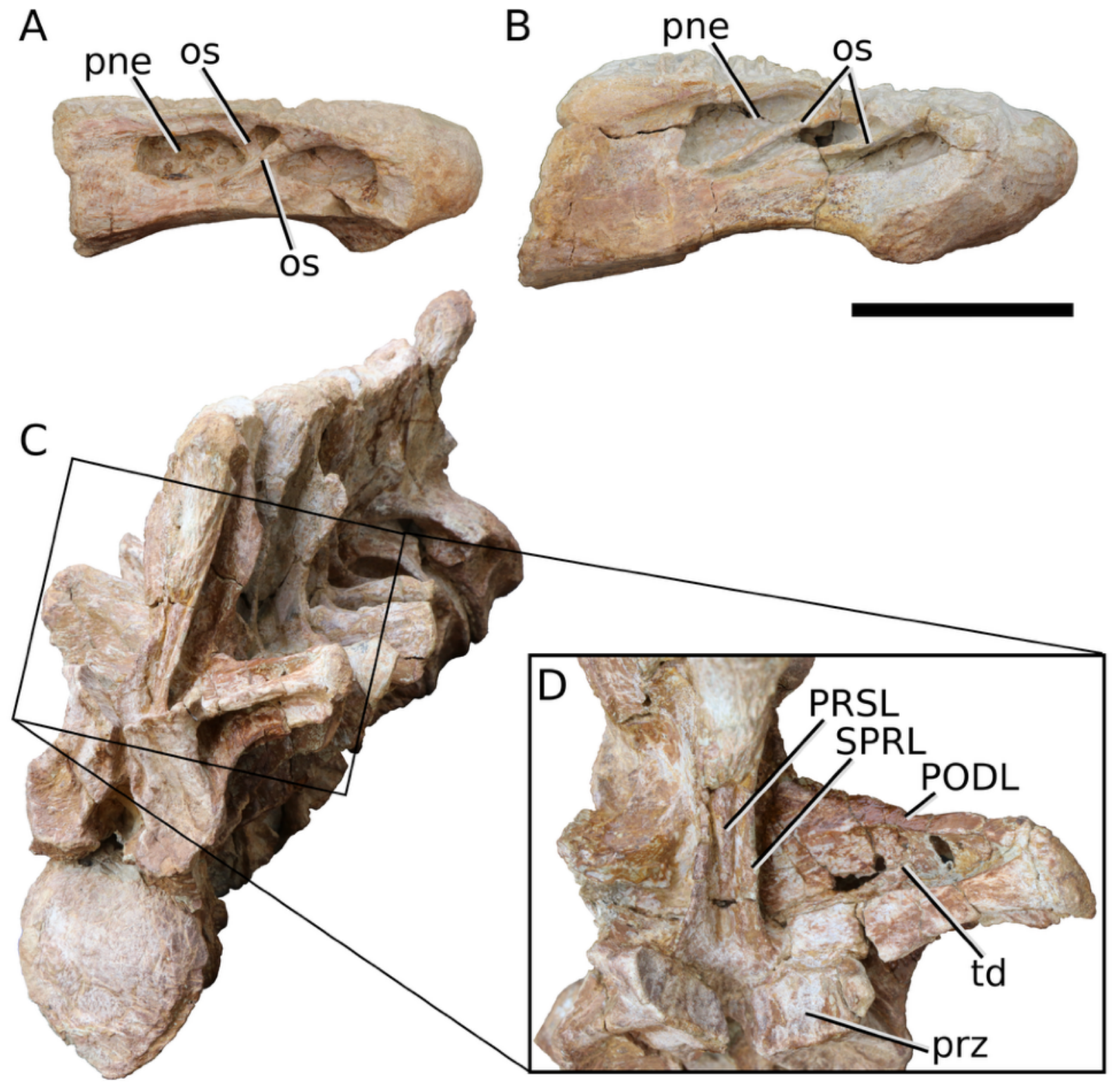

Figure 35. Pneumatic features in Bellusaurus (IVPP V17768). A, B, ?middle cervical vertebral centra in right lateral view. $\mathrm{C}$, last dorsal neural arch (missing its centrum) in articulation with the complete sacral series and first two caudal vertebrae in anterodorsolateral view. D, close-up of the neural spine and left diapophysis of the last dorsal vertebra. Abbreviations: os, oblique strut; pne, pneumatic excavation; PODL, postzygodiapophyseal lamina; PRSL, prespinal lamina; prz, prezygapophysis; SPRL, spinoprezygapophyseal lamina; td, trough-like depression of the diapophysis. Scale bar: A, B, D = $5 \mathrm{~cm}$. 


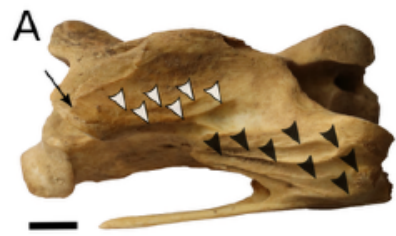

$\mathscr{L}=$ epipophysis, with insertion scars for $\mathrm{m}$. longus colli dorsalis and $\mathrm{m}$. ascendens cervicalis

$\nabla=$ attachment scars for $\mathrm{mm}$. intercristales

$\boldsymbol{\nu}=$ attachment scars/rugosities for $\mathrm{m}$. ascendens cervicalis and $\mathrm{mm}$. intertransversarii

$\nabla=$ accessory strut of pneumatized spinodiapophyseal fossa
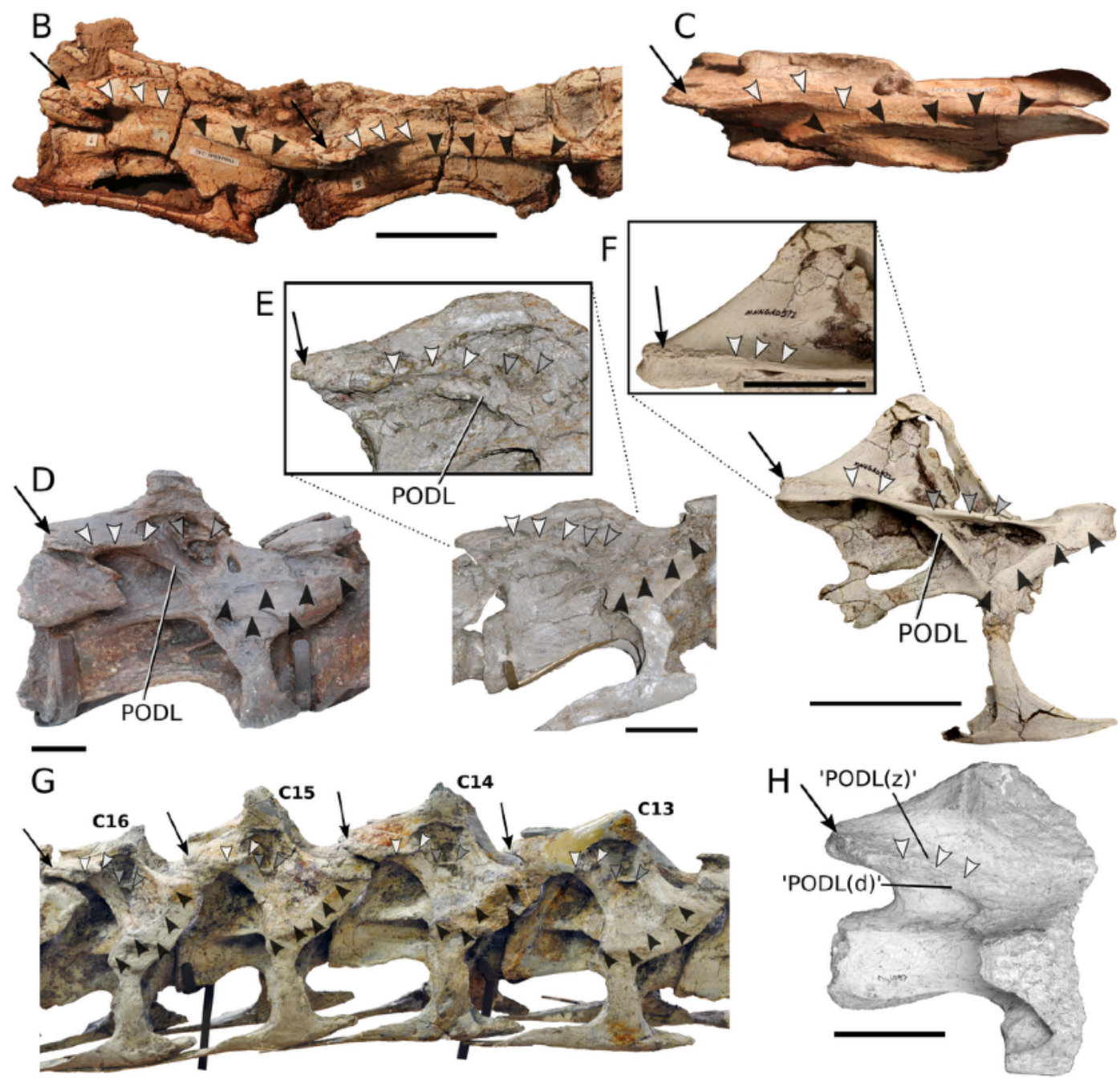

Figure 36. Proposed homology of osteological structures related to the EPRL. Colored arrowheads describe muscular and pneumatic morphologies of the lateral surface of the neural arch, as informed by surface texture and comparisons to extant archosaurs (see text). Naming of inferred musculature uses avian terminology. Arrowheads labelling inferred attachment sites of cervical musculature do not show the entire attachment territory; instead, these are meant to emphasize the anterior projection of muscle scars that are contiguous with the epipophysis (white arrows), and to highlight the physical separation of this projection from rugosities that occupy the lateral surface of the prezygapophyseal ramus and diapophysis (black arrows) in sauropods. A, mid-cervical of Struthio (uncatalogued specimen) in right dorsolateral view; B, cervicals 5-6 of the holotype of Sarahsaurus aurifontanalis (TMM 43646-2) in right lateral view; C, isolated middle or posterior cervical neural arch of the paratype of Sarahsaurus aurifontanalis (TMM 
43646-3) in left dorsolateral view (reversed); D, cervical 12 of Klamelisaurus (IVPP V9492) in right lateral view; E, cervical 8 of Euhelopus (PMU 233) in right lateral view, with close up showing the epipophysis and spinodiapophyseal fossa; F, cervical 10 of Nigersaurus (MNBH GAD 512) in right lateral view, with close up showing the epipophysis and extent of the anterior epipophyseal muscle scar; G, cervicals 13-16 of Euhelopus (PMU 233) in left dorsolateral view (reversed); $\mathrm{H}$, mid-cervical vertebra of Uberabatitan (CPP-1023-UrHo) in left lateral view (reversed), modified from Salgado \& de Souza Carvalho (2008, fig. 6). Abbreviations: C, cervical; PODL, postzygodiapophyseal lamina (' $d$ ' denotes diapophyseal segment and ' $z$ ' denotes zygapophyseal segment, after the interpretation of Salgado \& de Souza Carvalho [2008]). Images of Euhelopus in (E) and (G) used with permission of B. Kear. Permission to reproduce the image of Uberabatitan in $(\mathrm{H})$ was provided by John Wiley and Sons, Inc., (C) The Palaeontological Association. Scale bars: A = 1 cm: B, C = 5 cm; D-F, H $=10 \mathrm{~cm}$. 


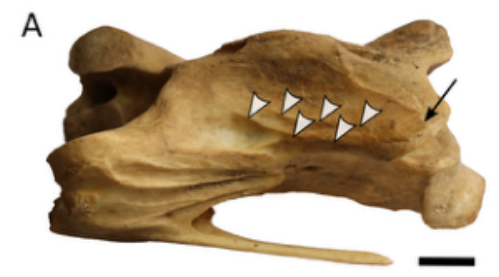

B

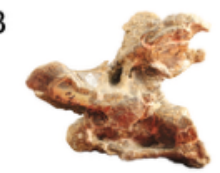

F
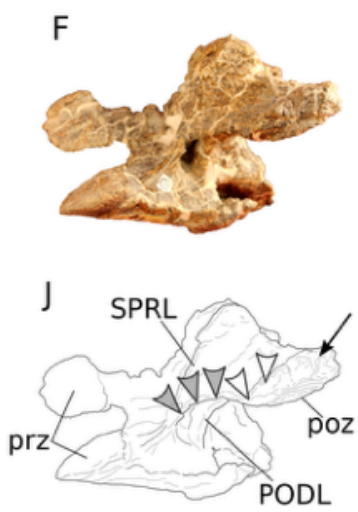

C
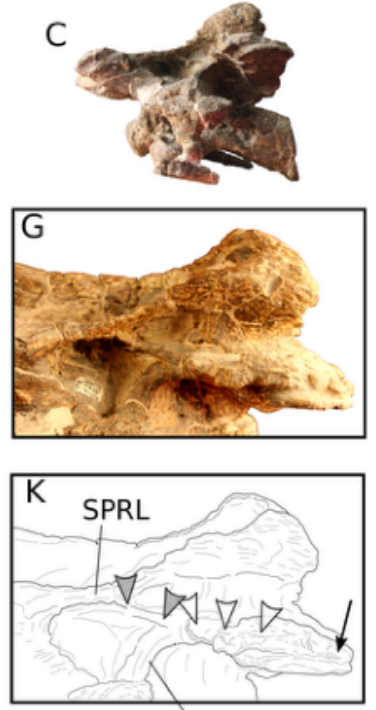

PODL $\swarrow=$ epipophysis, with insertion scars for $\mathrm{m}$. longus colli dorsalis and $\mathrm{m}$. ascendens cervicalis

$\nu=$ attachment scars for $\mathrm{mm}$. intercristales

$\nabla=$ accessory strut of pneumatized spinodiapophyseal fossa
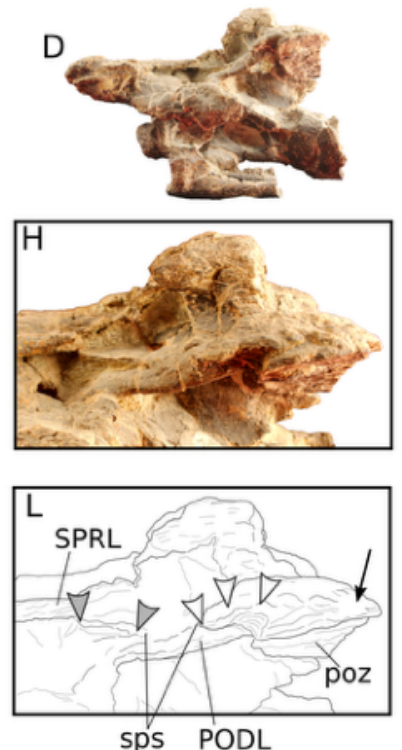
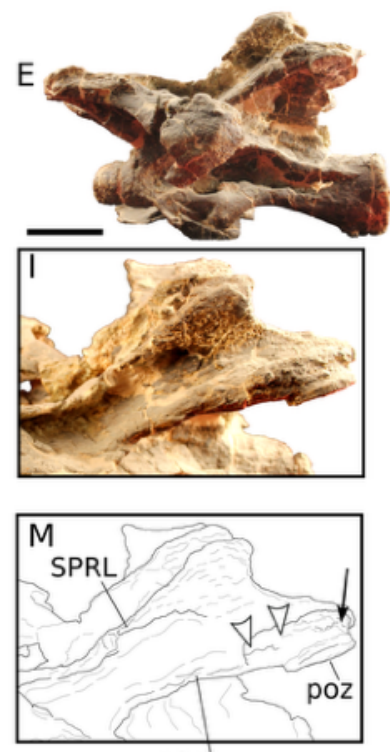

PODL

Figure 37. Structures sometimes considered to constitute components of a single EPRL exhibit serial dissociation in Camarasaurus lewisi. A, mid-cervical of Struthio (uncatalogued specimen) in right dorsolateral view (reversed); B-E, cervicals 3-5 and 7 of Camarasaurus lewisi (BYU 9047) in right lateral view (reversed); F-I, close-up views of cervicals 3-5 and 7 of Camarasaurus lewisi (BYU 9047); J-M, schematics of close-up views of cervicals 3-5 and 7 of Camarasaurus lewisi (BYU 9047). Abbreviations: PODL, postzygodiapophyseal lamina; PRDL, prezygodiapophyseal lamina; SPRL, spinoprezygapophyseal lamina; sps, smooth pneumatic surface. Scale bars: $A=1 \mathrm{~cm} ; \mathrm{B}-\mathrm{E}=10 \mathrm{~cm}$. All other images not to scale. 